DoE/RC/92526--T/4

\title{
RHEOLOGY OF COAL-WATER SLURRIES PREPARED BY THE HIGH-PRESSURE ROLL MILL GRINDING OF COAL
}

DOE Grant No. DE-FG22-92PC92526

Final Report

Prepared by

D. W. Fuerstenau and A. De University of California, Berkeley

Berkeley, California 94720

Prepared for

U. S. Department of Energy Pittsburgh Energy Technology Center

Pittsburgh, Pennsylvania 15236

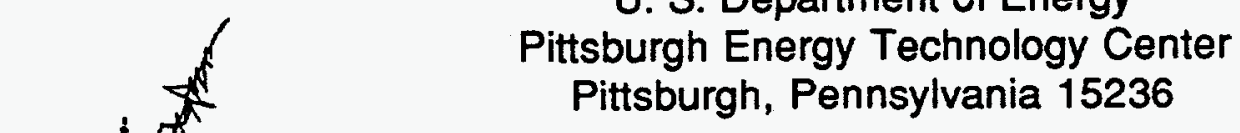

August 1996
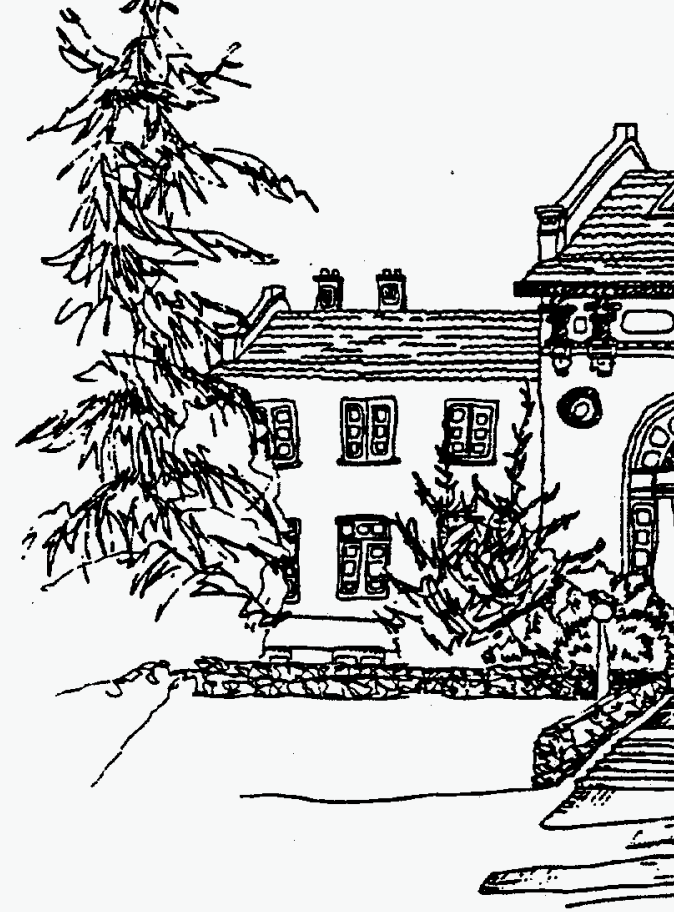

(1)

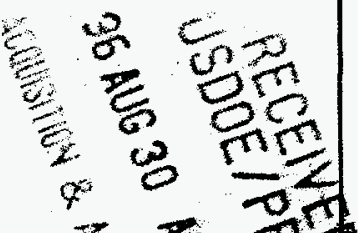

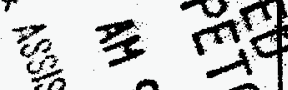

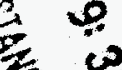

意为

累

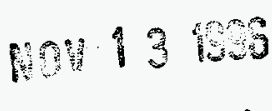 \\ resprob \\ OSTI}

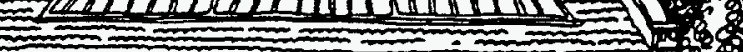

90

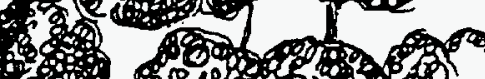




\section{DISCLAMMER}

Portions of this document may be illegible in electronic image products. Images are produced from the best available original document. 


\section{TABLE OF CONTENTS}

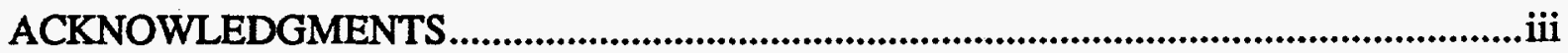

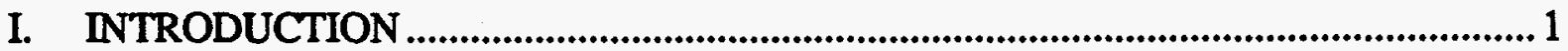

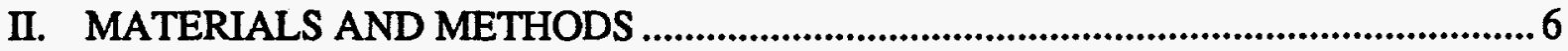

Coal Sample and Reagents ..........................................................................................6

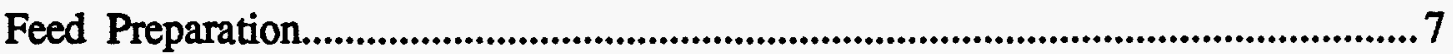

High-Pressure Roll Mill Grinding.............................................................................. 7

Experimental Procedure ................................................................................. 8

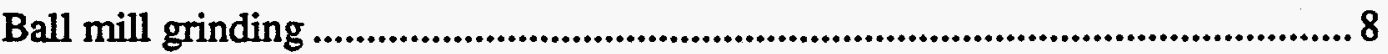

Experimental Procedure ...................................................................................

High-Pressure Roll Mill/Ball Mill Hybrid Grinding........................................... 10

Preparation of Coal-Water Slurries and Rheological Measurements ................... 10

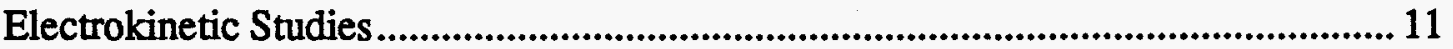

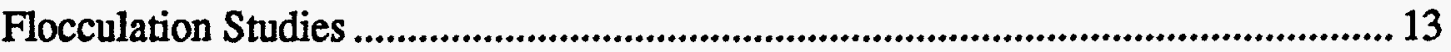

Contact Angle Measurement........................................................................................... 14

III. RHEOLOGY OF COAL-WATER SLURRIES PREPARED WTTH BALL MILL

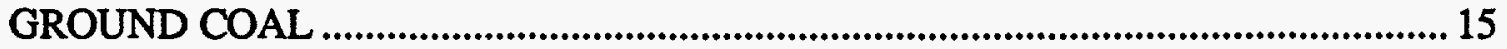

Electrokinetic behavior of Coal-Water Slurries............................................................. 16

Electrokinetic Behavior of Coal-Water Slurries at Low Suspension

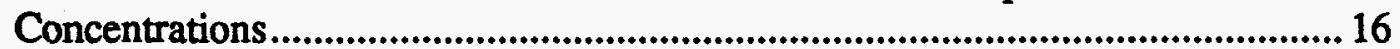

Electrokinetic Behavior of Moderately Concentrated Coal-Water Slurries......... 23

Effect of Chemical Additives on the Rheology of Coal-Water Slurries..................... 28

Effect of Chemical Additives on the Aggregation Stability in Coal-Water

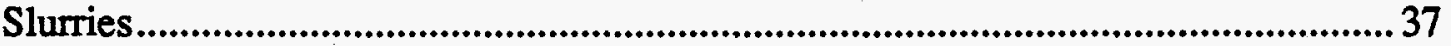

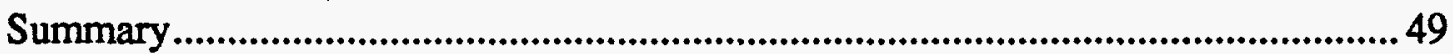

IV. HIGH-PRESSURE ROLL MILL GRINDING AND RHEOLOGY OF SLURRIES .... 51

High-Pressure Roll Mill Grinding of Coal ........................................................52

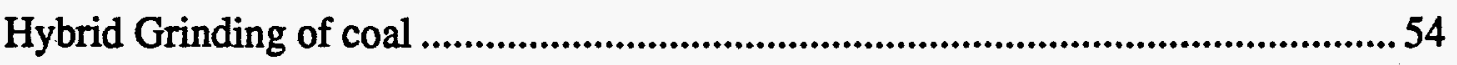

Kinetics of Open Circuit Hybrid Grinding of Coal...............................................55

Hybrid Batch Locked-Cycle Grinding ...............................................................58

Simulation of Hybrid Locked-Cycle Grinding Tests...............................................62

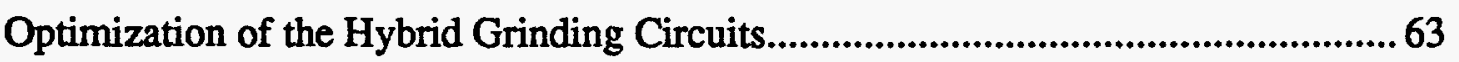

Effect of High-Pressure Roll Mill Grinding of Coal on the Slurry Rheology ..........69

Influence of Grinding Environment in the Ball Mill on the Energy Utilization and the Rheology of Slurries ................................................................................. 73

The Effect of Chemical Additives on the Rheology of Coal-Water Slurries.............. 78

Effect of Co-addition of Sodium hexametaphosphate ........................................... 79 


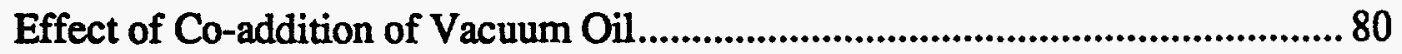

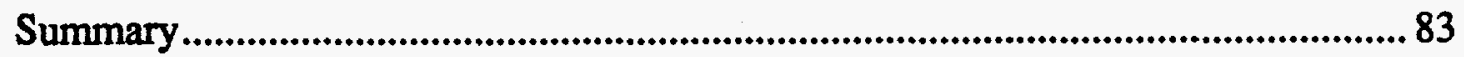

V. PACKING CHARACTERISTICS OF SOLIDS AND RHEOLOGY OF

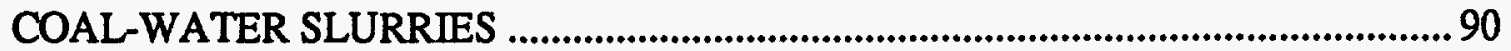

Relationship Between the Packing Density of Feed and the Viscosity of Slurry....... 90

Effect of Mixing Feed Distributions on the Packing Density of Coal Feed and the Rheology of Coal-Water Slurries..............................................................................91

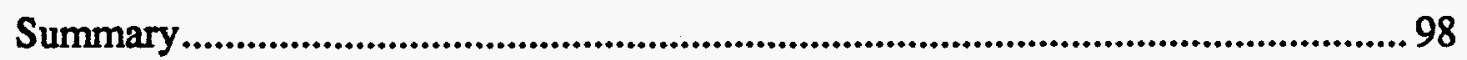

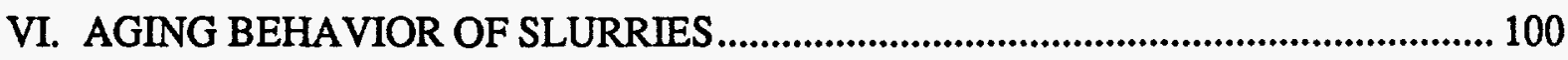

Influence of Iron on the Rheology of Coal-Water Slurries ......................................... 101

Spectroscopic Studies of Iron Interaction with Complexing Reagents................ 104

Effect of Iron on the Electrokinetic Behavior of Pittsburgh No. 8 Coal............. 105

Control of Aging Behavior of Coal-Water Slurries ............................................... 109

Effect of Iron-complexing Reagents on the Rheology of Coal-Water

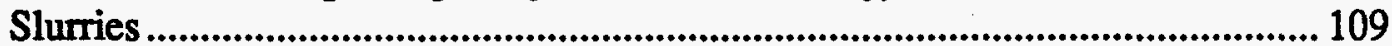

The Effect of Coal Cleaning on the Rheology of Slurries.................................. 118

Control of Aging Behavior of Slurries by the Addition of Vacuum Oil............. 121

Free Water Content and the Aging Behavior of Slurries .......................................... 122

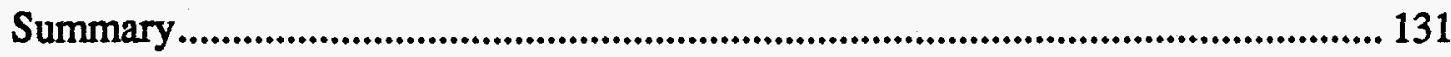

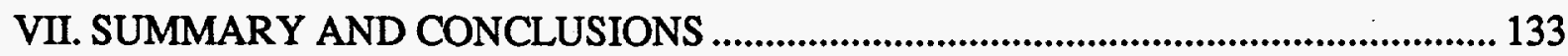

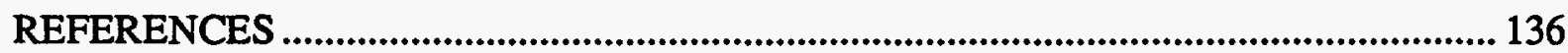




\section{ACKNOWLEDGMENTS}

This research, which was carried out in the mineral processing laboratories of the Department of Materials Science and Mineral Engineering, University of California at Berkeley, was sponsored by the Department of Energy, UCR Grant No. DE-FG2292PC92526. We wish to acknowledge the input to this research by three graduate student research assistants: M. Colic, F. Sotillo and J. Yang. Discussions with Professors T. W. Healy and P. C. Kapur are also acknowledged. 


\section{SECTION I}

\section{INTRODUCTION}

Greatly increased use of coal products is the most economical near-term solution to the problem of this country's excessive dependence on foreign oil imports because coal is the most abundant fossil fuel in the United States. On a uniform basis, U.S. coal resources are larger than the combined domestic resources of petroleum, natural gas and oil shale. A mineable coal reserve of over 300 billion tons, which is estimated to last for perhaps 300 years, requires that attention should be paid to the study of present and future economically feasible methods for coal beneficiation and its utilization as an energy source. The preparation of coal water slurries to replace fuel oil for direct combustion has become an important field in modern coal technology. The U.S. Department of Energy has planned or has underway several demonstration projects to burn coal-water slurries to replace fuel oil is attractive not only because there is an assured domestic supply of coal, but also on various technoeconomic grounds. Coal-water slurries combine the handing flexibility of fuel oil in power plants and various other industrial applications [1-4].

U.S. coal production in 1994 was approximately 938 million tons, of which 817 million tons ( $87.5 \%$ of the total) was burned by coal-fired power plants, to generate $56.2 \%$ of the total U.S. electricity [5]. However, sulfur emission from the combustion of coals from the eastern and midwestern regions of the United States poses air pollution problems, particularly acid rain phenomena. In order to effectively exploit our coal reserves without polluting the environment, complying with increasingly restrictive environmental regulations, removal of sulfur-bearing minerals from coal prior to combustion has become a very important aspect of coal preparation. Because most sulfur-bearing and ash-bearing minerals are finely disseminated in the coal matrix, fine grinding of coal is necessary in order to liberate and then separate them from the carbonaceous material by physical cleaning processes, such as froth flotation and oil agglomeration. Since coal cleaning processes are mostly water-based, the 
finely ground coal is already in an aqueous medium and, therefore, can directly be converted into a coal-water slurry fuel. This route could be easier and more economical than drying the material to a solid powder fuel for coal-fired power plants because it eliminates the high cost of fine coal dewatering and problems associated with the transportation and storage of dry coal fines.

In order to prepare coal-water slurries with sufficient stability, suitable rheological behavior and good combustibility, the raw coal must be ground to a fine size (say, a median size of $40 \mu \mathrm{m}$ or less) and the product should have a size distribution corresponding to dense packing density. Because fine grinding is an energy-intensive process, grinding costs represent a substantial portion of the total cost in advanced coal beneficiation and in coalwater slurry preparation. The total energy consumption for grinding one ton of coal to 80 percent finer than 200 mesh $(74 \mu \mathrm{m})$, the standard boiler feed size, is estimated to be in the range of 20 to $40 \mathrm{kWh} / \mathrm{t}[6,7]$. However, to obtain a ground coal with a median particle size of $10 \mu \mathrm{m}$, the total energy consumption is in the range of 75 to over $200 \mathrm{kWh} / \mathrm{t}$ depending on the hardness of the coal and the type of grinding mill used [7]. High grinding energy consumption and difficulty in obtaining a ground product size distribution corresponding to maximum packing density are major problems in the manufacture of coal-water slurries with conventional mills, such as the tumbling ball mill and stirred ball mill.

One of the most promising innovations in fine grinding in recent years has been the development of the choke-fed, high-pressure roll mill about fifteen years ago, and within very few years it has proven to be the most efficient grinding mill in the field of cement clinker grinding [8]. Experience with over 300 industrial units of high-pressure roll mills installed for fine grinding of cement raw material, cement clinker, slags, limestone, minerals and ores shows that compared to conventional grinding mills, high-pressure roll mills (i) are lower in capital cost, (ii) occupy less space, (iii) show negligible wear rate, (iv) will accept feeds with varying moisture contents, and most importantly (v) cut down the grinding energy consumption by up to $50 \%$ or even more. 
Some 15 years ago, Professor Klaus Schonert began his investigations of particle-bed comminution [9] which resulted in the construction of a new type of mill for fine grinding the high-pressure roll mill. Optimal energy utilization in the comminution of brittle solids is achieved when a single particle is broken down slowly under pure compression. The next most efficient method for particle breakage is through the compressive loading of a bed of particles in a piston-die arrangement. In this mode, comminution occurs primarily by very high localized stresses generated within the particle bed. To carry out particle-bed breakage continously, Schonert conceived of a device to carry out this process continuously and invented the high-pressure roll mill. Figure 1.1 shows a schematic representation of this mill, which is comprised of two counter-rotating rolls mounted on a strong frame. The bearings of

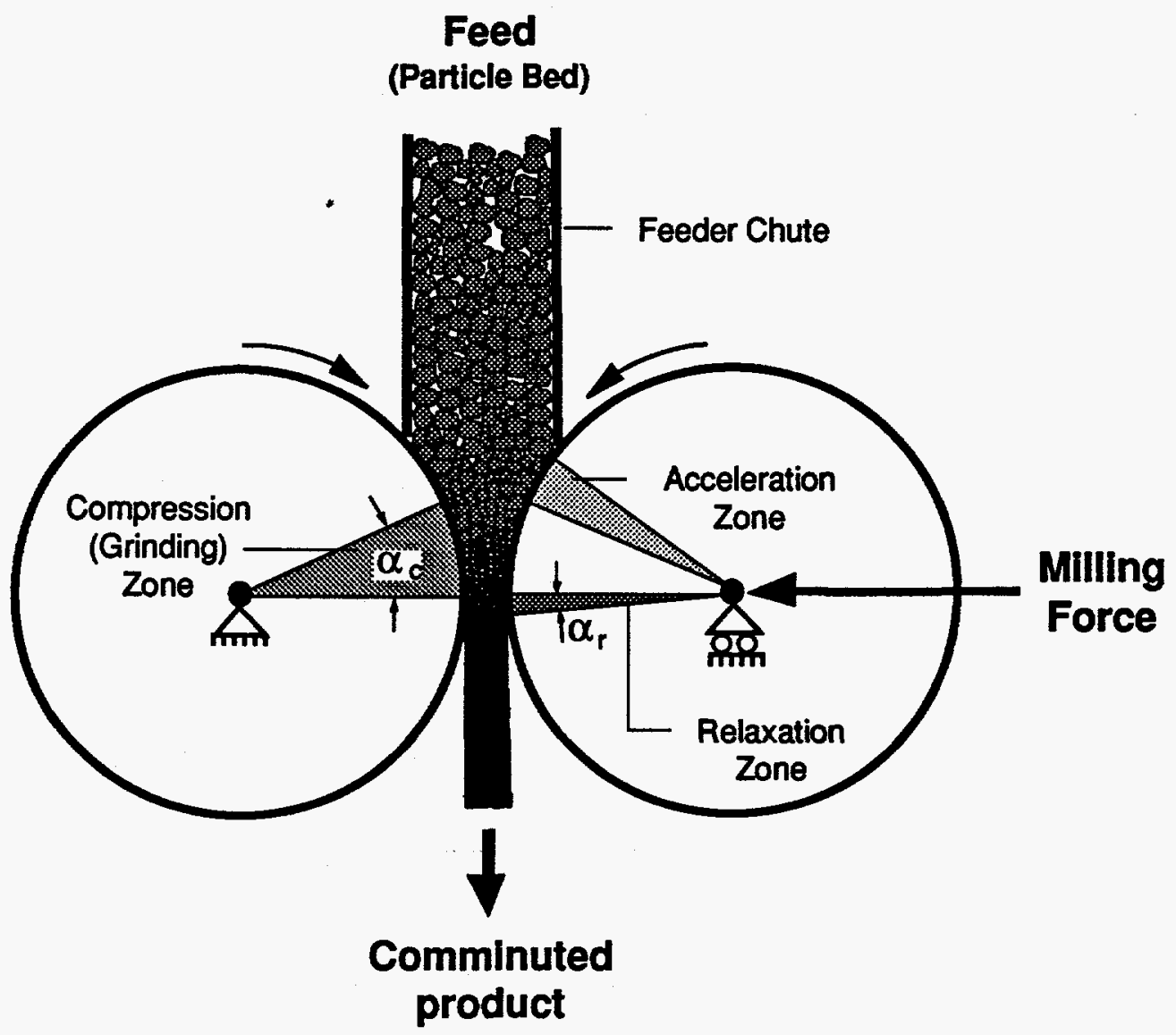

Figure 1.1. Schematic drawing of a choke-fed high-pressure roll mill, showing the particle bed, its compaction between the rolls, and the exiting comminuted product. 
one roll are fixed, while the other roll can slide laterally for adjustment of the gap. In a laboratory device, the milling force is applied by hydraulic or spring press loading of the moveable roll. The rolls are choke-fed from a feed hopper mounted directly above the roll gap. The ground product comes out in the form of lightly compacted flakes which are broken up by a de-agglomerator, such as by passing the material quickly through a ball mill or a hammer mill, or by simply stirring it in water. The mill can be used with dry as well as with moist-feed (up to $30 \%$ water content).

Compared to a ball mill grinding circuit, the main advantage of the high-pressure roll mill system is its low energy consumption, low initial capital investment, and the small installation space required. The industrial application of high-pressure roll milling in the cement grinding was started by positioning the high-pressure roll mill before a ball mill grinding system to act as a booster [10]. This resulted in an energy saving of at least $20 \%$ and at the same time a $30 \%$ increase in production [8]. An important problem is optimizing the relative energy expenditure between the roll mill and ball mill steps in this two-stage comminution system.

The effects of chemical additives on the rheological properties of coal-water slurries have been studied by many researchers [11-16], with some of the results obtained by different researchers being mutually inconsistent. Chemical additives affect many properties of coal particles in a slurry such as the zeta potential, adsorbed layers, solvation layers and the state of agglomeration / dispersion. The addition of anionic and nonionic surfactants can significantly improve the sedimentation stability and reduce the viscosity. The surface properties of the coal itself can also affect the properties of coal-water slurries [17]. It is known that coal is a heterogeneous substance with an extensive pore structure. The carbonaceous and hydrocarbon components contribute to the hydrophobic character of the coal, whereas oxygen in the polar functional groups (- $\mathrm{COOH},-\mathrm{COH},-\mathrm{C}=\mathrm{O}$, etc.) and the mineral matter contribute to the hydrophilic behavior of coal. The hydrophobic/hydrophilic mixed character of coal determines the nature of interaction between the surface of coal and molecules of water and 
chemical additives and hence the rheological behavior of coal-water slurries. Lower rank coals, which have higher $\mathrm{O} / \mathrm{C}$ ratios and equilibrium moisture, exhibit higher viscosities [18]. An explanation for this is that water is readily absorbed into the pores of hydrophilic coals and as a consequence reduces the amount of water available for flow. For the same reason, oxidation of coal also increases the viscosity of slurries prepared with that coal. Clean coal generally results in a decrease in viscosity for a given solids concentration of the slurry[19].

The principal objective of this research work was to systematically investigate the energy consumption, and the stability and rheology of coal-water slurry fuel prepared with a laboratory-scale, choke-fed, high-pressure roll mill, and to compare the results with slurry prepared with coal from a conventional grinding mill (such as the ball mill). An additional objective was to investigate the effect of the addition of reagents on the surface properties of coal and on both the short-term and long-term rheological behavior of coal-water slurries. Control of the effects of coal oxidation on slurry rheology was studied in detail. 


\section{SECTION II}

\section{MATERIALS AND METHODS}

The coal sample, chemical additives, and various experimental techniques used for the preparation and study of the behavior of coal-water slurries are presented in this section. A description of the coal sample and the reagents is followed by the feed preparation procedure, which includes the descriptions of the grinding mills and the grinding procedure. The various measurement techniques are also described.

\section{COAL SAMPLE AND REAGENTS}

The coal sample used for this research project, a Pittsburgh No. 8 bituminous coal from Warnock, Ohio, was supplied by R\&F Coal Co. The proximate analysis of the coal on a dry basis was: $35.7 \%$ volatile matter, $52.5 \%$ fixed carbon and $11.8 \%$ ash. The moisture content of the as-received coal was 2.3 percent.

The following reagents were used as dispersants for the preparation of coal-water slurries:

1) Triton $X$ series of nonionic surfactants $\left[\mathrm{C}_{8} \mathrm{H}_{17}-\mathrm{C}_{6} \mathrm{H}_{4}-\left(\mathrm{O}-\mathrm{CH}_{2}-\mathrm{CH}_{2}\right)_{n}-\mathrm{OH}\right.$, with different number of ethoxy groups: Triton $X-100$ ( $n=9-10)$, Triton $X-102$ $(n=12-13)$, Triton X-165 $(n=16)$, Triton X-305 $(n=30)$, and Triton $X-405$ $(n=40)$

2) An anionic surfactant, 2-isothiouronium-ethane phosphonic acid (2-TEPA), with molecular weight of 185

3) A cationic surfactant, 2-trimethylammonium-ethane isothiouronium dichloride (TMAE), with molecular weight of 235

4) A polyelectrolyte, sulfonated napthalene condensate (CoalMaster A-23-M), with molecular weight of about 3600 .

In addition, sodium hexametaphosphate and vacuum oil were used as secondary modifiers of the slurry viscosity. Sulfosalicylic acid and 2,3-dihydroxypyridine were used as iron-complexing agents. 


\section{FEED PREPARATION}

Fines used for the preparation of coal-water slurries were produced either by ball mill grinding or by high-pressure roll mill/ball mill hybrid grinding. Feed for the high-pressure roll mill and ball mill grinding experiments was distributed in size, the entire feed being finer than 8 mesh. In order to ensure that all samples had the same moisture content, the first step in the sample preparation procedure for coal involved drying the raw coal in a mechanical convection oven at $40^{\circ} \mathrm{C}$ for 15 hours prior to comminution.

Preparation of the feed with the required size characteristics involved stage-wise size reduction of the oversized material. Particles larger than 1-inch were first crushed in a laboratory jaw crusher set at a 3/4-inch gap and then stage-crushed in a standard laboratory roll crusher, while taking care to minimize the formation of excessive amounts of fines. The product of the roll crusher was dry-screened on a 8-mesh screen to yield the minus 8 mesh distributed feed. The oversize was crushed again in the roll crusher at a reduced roll gap setting.

\section{High-pressure roll mill grinding}

Our laboratory-scale, choke-fed high-pressure roll mill is comprised of two steel rolls $200 \mathrm{~mm}$ diameter $\times 100 \mathrm{~mm}$ width, one of which is in a laterally sliding pillow block. This movable pillow block is subjected to a milling force, which is generated inside four cylindrical force cages. Each force cage contains an assembly of eight disc springs, a piston, a load cell and has a spring-bolt at its end. While the cage is fixed to the frame, the piston and load cell can move freely. Tightening the spring bolt compresses the disc-spring and generates the milling force which is transmitted via the load cells to the movable pillow block. A milling force of up to $150 \mathrm{kN}$ can be applied to the movable roll by a spring-loaded press. The rolls are driven by a $50 \mathrm{hp}$ variable speed dc motor. The roll speed can be varied between 0.1 to $3 \mathrm{~m} / \mathrm{s}$ and the roll gap from 0 to $6 \mathrm{~mm}$. Mill torque is measured by a $1000 \mathrm{Nm}$ torque trans- 
ducer attached to the drive shaft and is linked with a computer system that is programmed to operate the mill while recording the mill torque, roll speed and roll gap simultaneously.

\section{Experimental Procedure}

Minus 8-mesh feed was ground in the high-pressure roll mill at different levels of specific energy input The amount of energy invested in grinding per pass through the rolls ranged from 0.5 to about $3.7 \mathrm{kWh} /$ ton. The specific energy input was increased by increasing the load on the spring-loaded press. The roll speed varied in the range of 15 to $20 \mathrm{rpm}$ and the feed rate varied in the range of about 20 to 100 grams per second depending on the milling force applied. The time during which samples were taken in each grinding experiment was 5 seconds during which the roll speed, roll gap and the mill torque were continuously measured. At the end of each run, the product was weighed and transferred into sample bags for size analysis or to serve as the feed to the ball mill in hybrid grinding studies.

\section{Ball mill grinding}

The ball mill system used for studying the energetics of fine grinding has been specially designed and instrumented for accurate measurement of energy consumption under standardized grinding conditions. A detailed description of this mill was presented by Yang, Mempel and Fuerstenau [20]. The dimensions of the two different stainless steel ball mills used are 11.5 inches $(292 \mathrm{~mm})$ long by 10 inches $(254 \mathrm{~mm})$ inner diameter, and 5.75 inches (146 mm) long by 5 inches $(127 \mathrm{~mm})$ inner diameter. The smaller mill is scaled to one-half of the diameter and length of the larger mill. Both mills are equipped with eight lifter bars each being 6.30 and $3.15 \mathrm{~mm}$ in height for the large and small mills, respectively. The mill, which is mounted on pillow block ball bearing rollers to support the mill shell, is driven by a variable speed drive (Graham Variable Speed Drive, Model No. 200MT2.8, 1-hp motor, 0 to $230 \mathrm{rpm}$ output) through a MCRT Torquemeter (Model 2802T, Range $500 \mathrm{in}-\mathrm{lb}$, manufactured by S. Himmelstein \& Co.). Data are acquired by measuring the voltage response from a torque 
transducer that is positioned between the drive assembly and the mill and is interfaced with a computer system. Mill speed, grinding time and mill torque are recorded simultaneously and the energy expended, in joules, is computed from the torque and mill speed data.

The grinding media for the 10-inch ball mill consist of $30 \mathrm{~kg}$ of one-inch $(25.4 \mathrm{~mm})$ diameter stainless steel balls which occupy about 50 percent of the mill volume at rest, while the grinding media for the 5 -inch ball mill consist of $3.75 \mathrm{~kg}$ of one-half-inch $(12.7 \mathrm{~mm})$ diameter stainless steel balls occupying 50 percent mill volume at rest. The 10 and 5-inch mills were run at 54 and $76 \mathrm{rpm}$, respectively, which corresponds to 60 percent of their critical speed (that is, the speed at which the balls would centrifuge).

Besides the torque ball mills, a third stainless steel ball mill, 10 inches long and 8 inches in internal diameter, was also used for the production of minus $\mathbf{2 0 0}$ mesh feed for the preparation of slurries. The grinding media charge comprised of $8.62 \mathrm{~kg}$ of 1-inch diameter steel balls, $5.18 \mathrm{~kg}$ of 0.75 -inch steel balls and $2.85 \mathrm{~kg}$ of 0.5 -inch steel balls, total weight being $16.65 \mathrm{~kg}$ and occupying about 45 percent of the mill volume at rest. The mill was run at $56 \mathrm{rpm}$, which is $60 \%$ of the critical speed, with a coal charge of 500 grams.

\section{Experimental Procedure}

The amount of coal sample charged to the 10 and the 5-inch ball mills for all grinding experiments was kept fixed at $1.5 \mathrm{~kg}$ and 160 grams, respectively, which translates to about 100 percent filling of the interstices of the ball charge at rest. The mills were layer-loaded with balls and feed, that is one layer of balls and then one layer of coal particles were carefully placed in the mill. Subsequently, the mill was closed tightly and mounted on the rollers. The grinding tests were controlled by the data acquisition software, run on an IBM-PC/AT computer, which would run the ball mill for the specified length of time while acquiring torque and mill speed data at specified intervals. The mill was discharged after completion of the grinding test onto a $1 / 2$-inch screen to separate the ball charge from the ground product. Approximately 150 grams of ground product was then collected by cone-and-quartering for 
size analysis. Unless specified otherwise, the size distribution was obtained by dry sieving for 20 minutes in a Ro-Tap machine.

The size distributions of the minus 200-mesh samples, used for the preparation of coalwater slurries, were determined using a Leeds Northrup Microtrac Particle-Size Analyzer.

\section{High-pressure roll mill/ball mill hybrid grinding}

One of the primary objectives of this research project is to investigate the rheology of the coal-water slurries prepared from coal fines generated by high-pressure roll mill grinding. High-pressure roll mill grinding of coal results in briquetted product which has to be deagglomerated and further ground to obtain minus 200-mesh coal fines for the preparation of coal-water slurries. Ball mills have been used for the deagglomeration and grinding of the high-pressure roll mill product. Ball mill deagglomeration and grinding of the briquetted product has been carried out in open-circuit as well as in closed circuit configuration. Both the circuits were operated in the batch mode. Figures $2.1 \mathrm{a}$ and $2.1 \mathrm{~b}$ show the schematic representations of the open-circuit and closed-circuit grinding configurations, respectively. In open-circuit grinding, a fixed weight of the high-pressure roll mill product is ground in the ball mill until the coal charge has reached a desired fineness (approximately $95 \%$ minus 200 mesh). In closed-circuit grinding mode, a fixed amount of the high-pressure roll mill product is first ground in the ball mill to, say, 40 percent minus 200 -mesh. The mill discharge is screened at 200 -mesh. The minus 200 -mesh fraction is the desired product. The screen oversize is mixed with fresh high-pressure roll mill product to make up the balance of charge to the ball mill.

\section{PREPARATION OF COAL-WATER SLURRIES AND RHEOLOGICAL MEASUREMENTS}

Slurries with different weight percentages of solids were prepared by mixing a coal sample with distilled water in the absence and presence of different reagents in a $250 \mathrm{ml}$ 


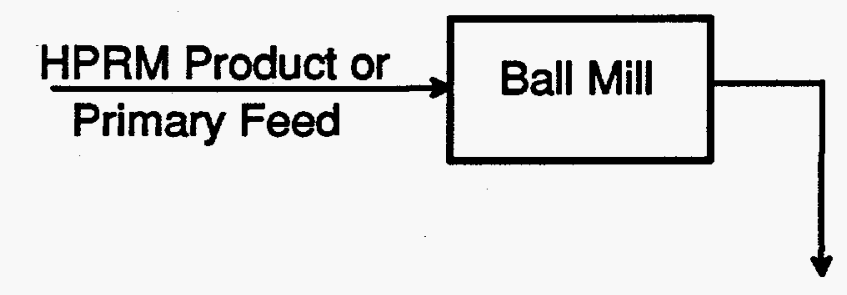

(a)

Product

(Minus 200 mesh)

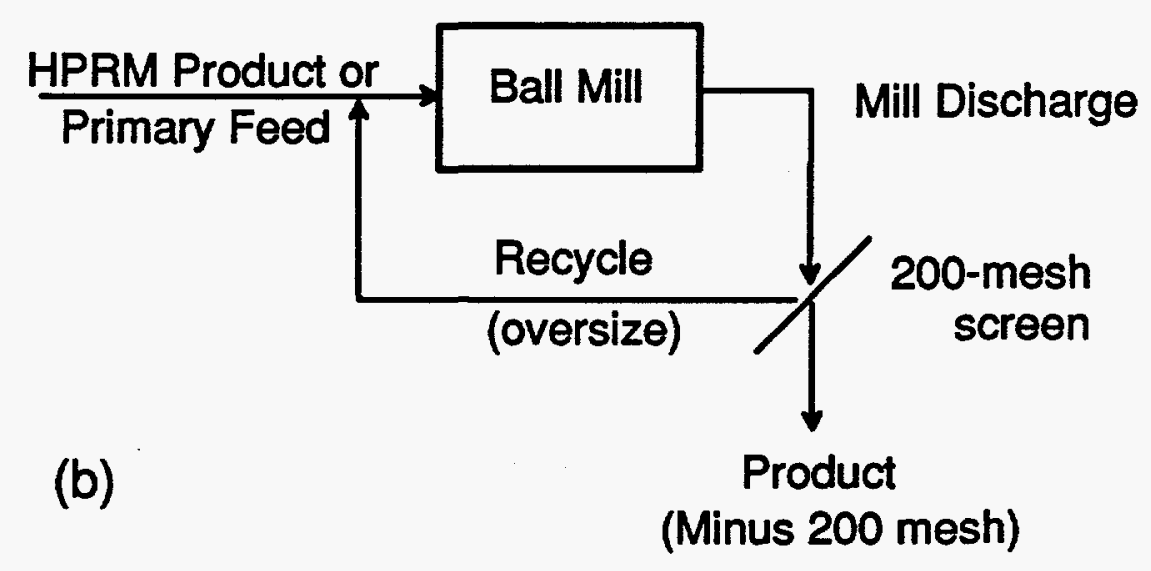

Figure 2.1. Schematic representation of (a) open-circuit and (b) closed-circuit two-stage hybrid grinding configurations.

container. In this mixing step, the suspension was stirred using a mechanical stirrer for five minutes at $1000 \mathrm{rpm}$. After mixing, the coal-water slurry was conditioned for 16 hours in a Lab-Line Instrument Environ-Shaker 3597 at $20^{\circ} \mathrm{C}$ and $200 \mathrm{rpm}$. Rheological measurements were carried out with a Hakke Rotovisco RV12 viscometer using a MV-DIN sensor system at a constant temperature of $20^{\circ} \mathrm{C}$. The torque measured by the viscometer was recorded as a function of the rotational speed of the sensor. The shear stress and the shear rate were then calculated and plotted (rheogram) to characterize the rheological behavior of the slurries.

\section{ELECTROKINETIC STUDIES}

The surface charge characteristics of coal particles in dilute suspensions were studied using traditional electrophoretic mobility measurements conducted with a ZetaMeter 3.0 
manufactured by ZetaMeter, Inc., New York. The voltage applied was normally 100 volts, and the mobility of the sample was taken as the average of twenty measurements. The mass concentration of suspensions used in the measurements was $0.01 \mathrm{~g} / 100 \mathrm{ml}$, and the ionic strength was adjusted with $0.001 \mathrm{M} \mathrm{NaNO}_{3}$.

The electrokinetic behavior of coal in moderately concentrated suspensions were studied using an acoustophoresis technique. For the acoustophoresis measurements, a Pittsburgh No. 8 sample (as-received) was dry ground for $\mathbf{3 0}$ minutes in a stainless steel ball mill to obtain a product $90 \%$ finer than 200 mesh. For each acoustophoresis test, $29.5 \mathrm{~g}$ of coal was placed in the Teflon cell of the Matec ESA 8000 apparatus. Since the cell has a capacity of $200 \mathrm{~cm}^{3}, 198 \mathrm{~cm}^{3}$ of triple-distilled water was added as make up water to obtain a slurry of $10 \mathrm{vol} \%$ solids content. Mixing of the slurry was performed in the Matec cell using a mechanical agitator at $1000 \mathrm{rpm}$ for five minutes, followed by dispersing it in an ultrasonic bath for five minutes. The cell containing the slurry was then equilibrated for sixteen hours in the Matec ESA 8000 apparatus. After this equilibration time, tritrations were performed. The $\mathrm{pH}$ was adjusted automatically by the Matec ESA 8000 computer controlled equipment using $0.1 \mathrm{~N} \mathrm{NaOH}$. During the equilibration and the measuring of a sample, argon gas was purged to avoid oxidation. At the end of each titration, the sample was discarded and a new sample was prepared following the same procedure, except that after the equilibration period, this second sample was filtered and the supernatant was returned to the cell for the measurement of the Electrokinetic Sonic Amplitude (ESA) of the supernatant under the same conditions as those used for the original sample. These ESA signals were used for the background corrections.

When acoustophoresis measurements of the filtered-and-washed samples were carried out, the samples required additional preparation steps. For the filtered sample, an as-received sample was equilibrated for 16 hours after which the suspension was filtered and the solid residue was dried under vacuum for $\mathbf{4 8}$ hours. The sample was then handled according to the procedure described above. In the case of the filtered-and-washed sample, the sample was 
washed after filtration with three times the cell volume of triple-distilled water. Then the sample was handled following the same experimental procedure delineated for the filtered sample.

The Matec ESA 8000 apparatus is able to measure the ESA signal, the $\mathrm{pH}$ and the conductivity and calculate the zeta potential of the suspension in the cell.

\section{FLOCCULATION STUDIES}

Flocculation studies were performed on coal-water slurries through such indirect measurements as determination of the relative sedimentation volume and the size distribution of flocs. For these two measurements, coal-water slurries were prepared following the same procedure described for the rheology measurements. The relative sedimentation volume both in absence and presence of chemical additives was determined by comparing the initial slurry height in a $30-\mathrm{cm}^{3}$ centrifuge tube with the height measured after centrifuging the suspension for 30 minutes at $16,000 \mathrm{rpm}$ in a Sorvall Superspeed RC2-B automatic refrigerated centrifuge.

For the size distribution of the flocs, a sedimentation technique using an Andreasen pipette apparatus was employed. A suspension containing $60 \mathrm{wt} \%$ solids, which had been conditioned for 16 hours at $20^{\circ} \mathrm{C}$, was diluted to $2-3 \mathrm{wt} \%$ solids and equilibrated at room temperature $\left(22^{\circ} \mathrm{C}\right)$. The diluted suspension was then gently agitated for 30 seconds before the sedimentation test was started. Samples of the suspension were obtained every 2.5 minutes for the first 15 minutes, and then the interval was doubled after every sampling until a total sampling period of 24 hours. The height of the suspension in the Andreasen pipette was also recorded during each sampling. The samples obtained at each time interval were dried and weighed, and from the weight, height and time data the Stoke's or sedimentation diameter was calculated, and the weight distribution was plotted as a function of the measured floc size to obtain the apparent particle size distribution. 


\section{CONTACT ANGLE MEASUREMENT}

The contact angle is defined as the angle measured across the liquid at the three-phase line of contact. The contact angles were measured using captive bubble methods or polished surfaces of coal specimens. For these measurements, a hand-picked coal lump was first cut produce a flat surface. The specimen was then dry polished with polishing papers ranging from 240 to 600 grit. The polished specimen was then washed with distilled water several times to remove traces of polishing powder. "Equilibrium" contact angles were measured using a Rame-Hart contact angle goniometer after a bubble had made contact with the sample surface. 


\section{SECTION III}

\section{RHEOLOGY OF COAL-WATER SLURRIES PREPARED WITH BALL MILL GROUND COAL}

The rheological behavior of concentrated coal-water suspensions is influenced by several factors: i) the surface characteristics of coal particles, ii) interaction between the coal surface and the aqueous phase, iii) particle-particle interaction (which depends on the particle shape and size distribution), iv) the solids content of the slurries, and finally, iv) the rest structure of the suspension (which is determined by some or all of the above factors).

For slurries to be used as a substitute for fuel oil, they should have good theological properties, namely, an apparent viscosity of $1000 \mathrm{mPa}$.s at a shear rate of $100 \mathrm{~s}^{-1}$, for transport through slurry pipelines, and high solids content which translates to high Btu content. Suitable modification and control of the above factors is essential for the production of coal-water slurries that satisfy these requirements. The surface properties and the interaction between coal particles and the aqueous phase can be modified by chemical additives, while the particle shape and size distribution might be altered by choosing the appropriate mode of size reduction for producing the fine particles for preparing the slurries. The shape and size distribution affects the packing characteristics of particles and hence has a direct bearing on the maximum achievable solids content of the slurry. Since size reduction by high-pressure roll milling is through confined particle-bed comminution, where the particlebed undergoes significant compaction, it is expected that high-pressure roll milling should result in higher packing densities of the resultant product particles.

The primary objective of this project has been to determine the effect of high-pressure roll milling of coal on the rheology of coal-water slurries. Quantitative assessment of these effects requires that a baseline be established against which the rheological behavior of coalwater slurries prepared with high-pressure roll milled coal can be compared. In this section, therefore, we will present the results of our investigation on the rheology of coal-water slurries prepared with fines produced by ball mill grinding of coal, studies which were carried 
out to establish the baseline.

The results of electrokinetic measurements carried out to delineate the surface characteristics of coal in the aqueous environment are also presented. The effect of chemical additives on modifying of the particle surface characteristics, particle-particle and particlewater interactions, and the rheology of coal-water slurries are also discussed.

\section{ELECTROKINETIC BEHAVIOR OF COAL-WATER SLURRIES}

The rheology of concentrated suspensions of non-interacting solid particles in a liquid phase is determined primarily by hydrodynamics, that is, the flow of liquid around the suspended particles. In case of coal-water slurries, the rheological behavior is also influenced by the particle-particle interactions due to the surface hydrophobicity and charge characteristics of the particles. Study of the electrokinetic behavior of coal particles in dilute and concentrated slurries might provide information not only on coal surface properties but also on the interaction between coal particles suspended in liquid. As shown by Fuerstenau and coworkers [21], such information might be extremely important in explaining coal-water slurry behavior. Depending on the rank of the coal, the degree of oxidation (of both coal and pyrite) and mineral matter content, the electrokinetic behavior of coal will vary significantly. In this investigation, the stability and viscosity of coal-water slurries will be correlated with the electrokinetic behavior in dilute and concentrated slurries.

\section{Electrokinetic Behavior of Coal-Water Slurries at Low Suspension Concentrations}

Traditional electrophoretic mobility measurements require samples of low solids concentration, which necessitates dilution of the original slurry (up to 10000 times). The measurements were conducted with a ZetaMeter 3.0 manufactured by ZetaMeter, Inc., New York. The voltage applied was normally 100 volts, and the mobility of the sample was taken as the average of twenty measurements. The mass concentration of suspensions used in the actual measurements was $0.01 \mathrm{~g} / 100 \mathrm{ml}$, and the ionic strength was adjusted with $0.001 \mathrm{M}$ $\mathrm{NaNO}_{3}$ 
An important parameter for characterizing the surface charge of coal particles in water is the condition when both the surface charge and the surface potential are zero (point of zero charge, PZC). Another condition of importance is when the Stern layer potential (potential at the first layer of counter ions) is zero, which is often called the isoelectric point (IEP). The electrokinetic or zeta potential, which has been measured in this study, is the potential at the shear plane where slip must occur when the solid moves relative to the liquid, and it is often assumed that it approximates Stern layer potentials. It is important to note that the behavior of the solid particles in liquid under this condition is governed by the charge density at the shear plane and, consequently, the zeta potential usually correlates well with stability. Zeta potentials can be changed through adsorption of inorganic or organic ions at the Stern plane. Therefore, cationic or anionic impurities, which are common in coal, can completely control the surface properties and stability of suspensions and slurries. Specific adsorption of cations or anions can change the location of the isoelectric point, or what we will term the point of zeta potential reversal (PZR). Specific adsorption of cations increases the PZR and the absolute value of the measured zeta potential, but the opposite is true for specific adsorption of anions (see Figure 3.1). An indifferent electrolyte is one that has no special affinity for the surface, such as $\mathrm{NaNO}_{3}$.

To find the PZR of clean Pittsburgh No. 8 coal, a sample of this coal was ground under an argon atmosphere to prevent oxidation, and thoroughly washed by filtration under high vacuum conditions. As illustrated by the results given in Figure 3.2, the PZR of clean coal was found to occur at $\mathrm{pH} 4.3$, which is not unusual for clean coal [21]. This was a significant decrease in the PZR when compared to the "as-received" sample of this coal, which had also been studied in the same experiment (PZR at pH 6.6). It is apparent that some specifically adsorbed cation controls the surface properties of the "as received" coal sample.

Preliminary analysis of filtrates and supernatants obtained after removal of the coal particles indicates that iron is a major cationic impurity in Pittsburgh No. 8 coal. Iron is probably produced by pyrite oxidation during storage of the coal for prolonged time (or under 


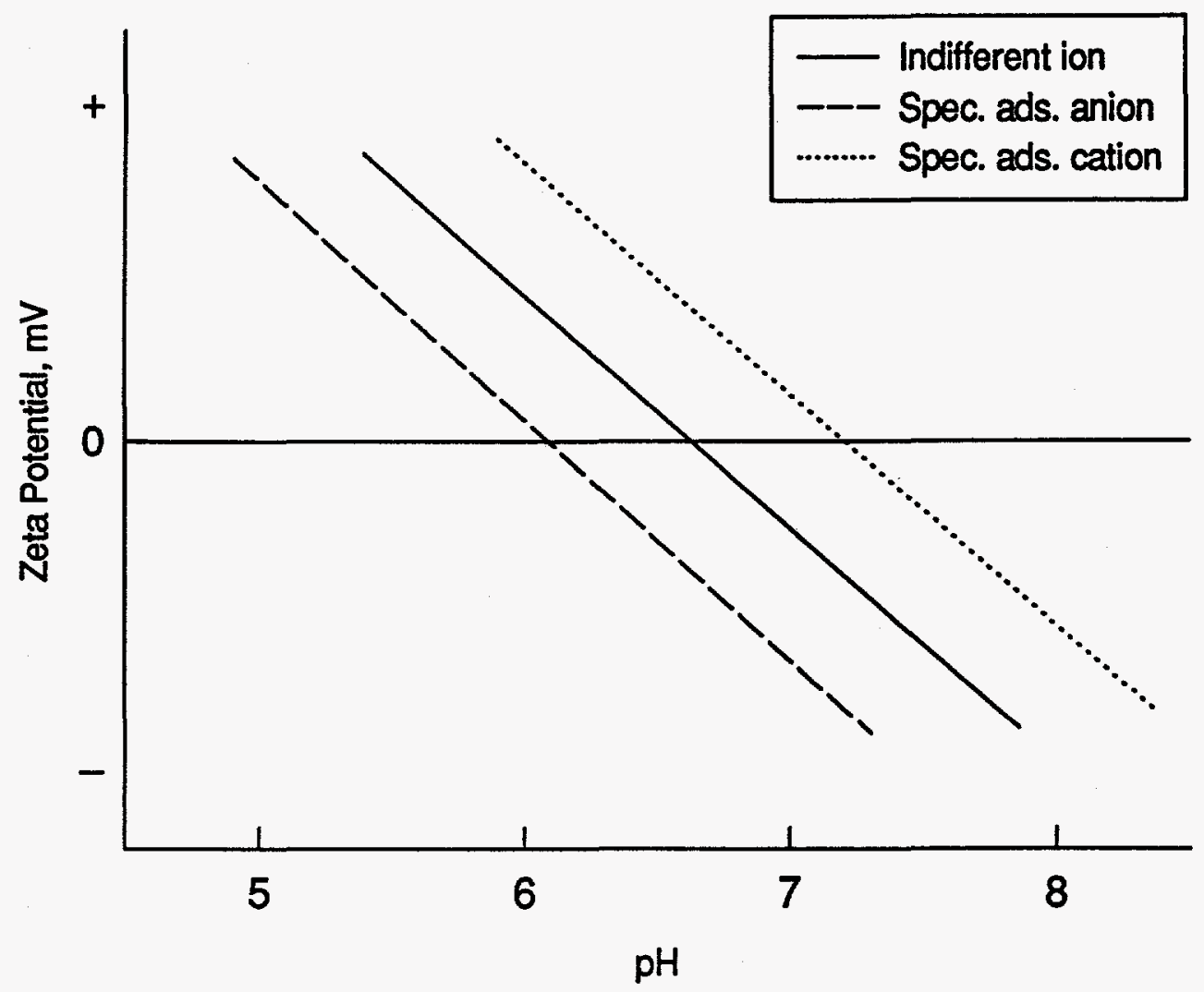

Figure 3.1. Schematic illustration of the effect of specific adsorption on the mobility and PZR of a colloid suspension.

the oxidizing conditions that results from contact with air). This dissolved iron specifically adsorbs on coal particles, increasing the $\mathrm{pH}$ of the PZR and absolute values of zeta potentials.

The effects of washing coal with water and iron complexing reagents on the zeta potential and PZR are shown in Figure 3.3. The PZR of "as-received" Pittsburgh No. 8 coal occurs at $\mathrm{pH}$ 7.1. Washing with water or sulfosalicylic acid, an anionic reagent known to complex iron, lowered the $\mathrm{pH}$ of the PZR to 4.8.

Electrokinetic measurements suggest that in the acidic $\mathrm{pH}$ range, slurries will have a weakly flocculated structure, that is, the slurries are expected to have a relatively high yield stress and exhibit shear thinning behavior. Figure 3.4a presents the flow curves of slurries prepared with ball milled coal fines, and Figure $3.4 \mathrm{~b}$ the apparent viscosity of the same systems. As suggested by the electrokinetic measurements, these slurries have very high 


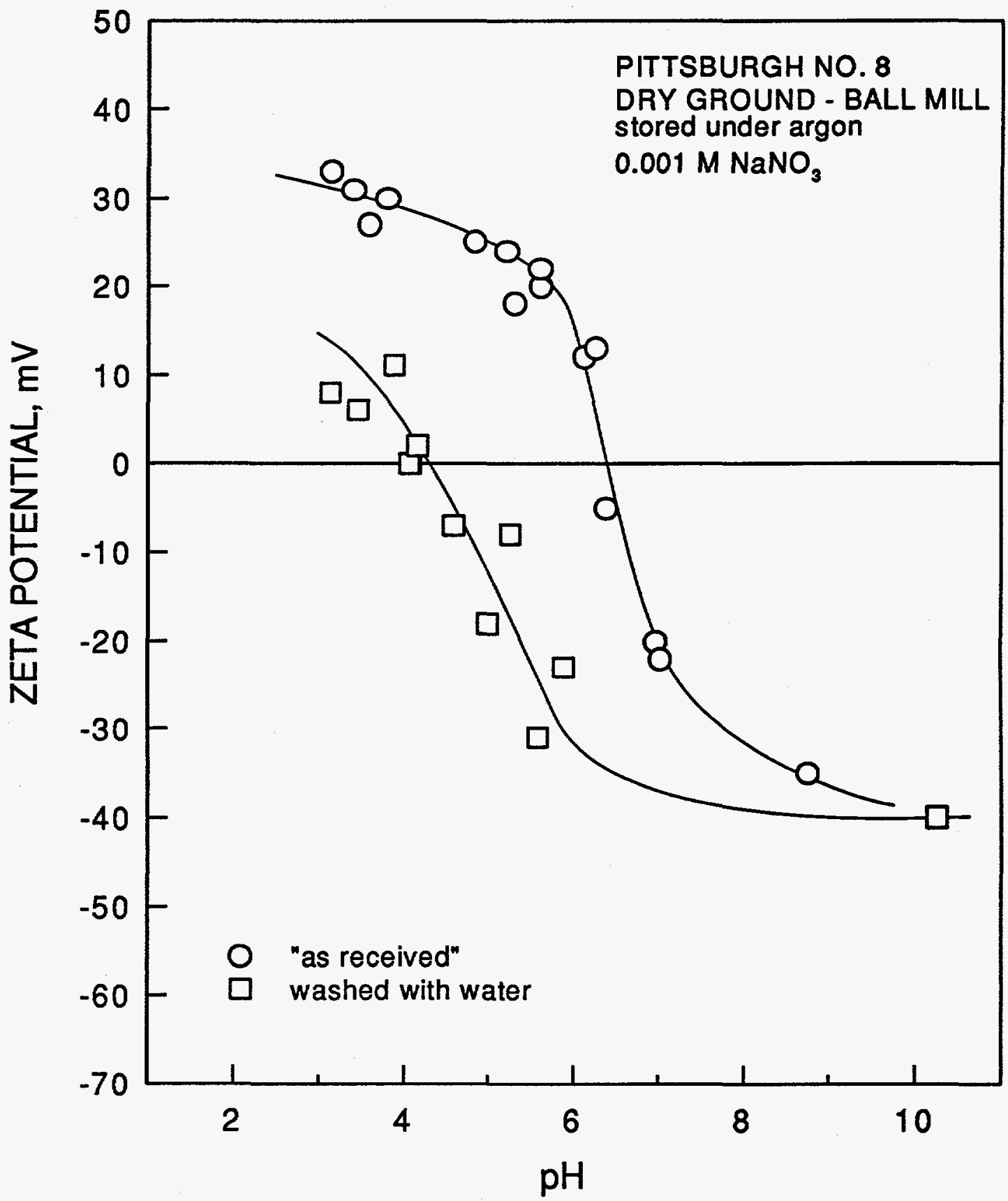

Figure 3.2. The zeta potential as a function of $\mathrm{pH}$ for unwashed and thoroughly washed Pittsburgh No. 8 coal samples prepared by ball milling and stored dry under argon. 


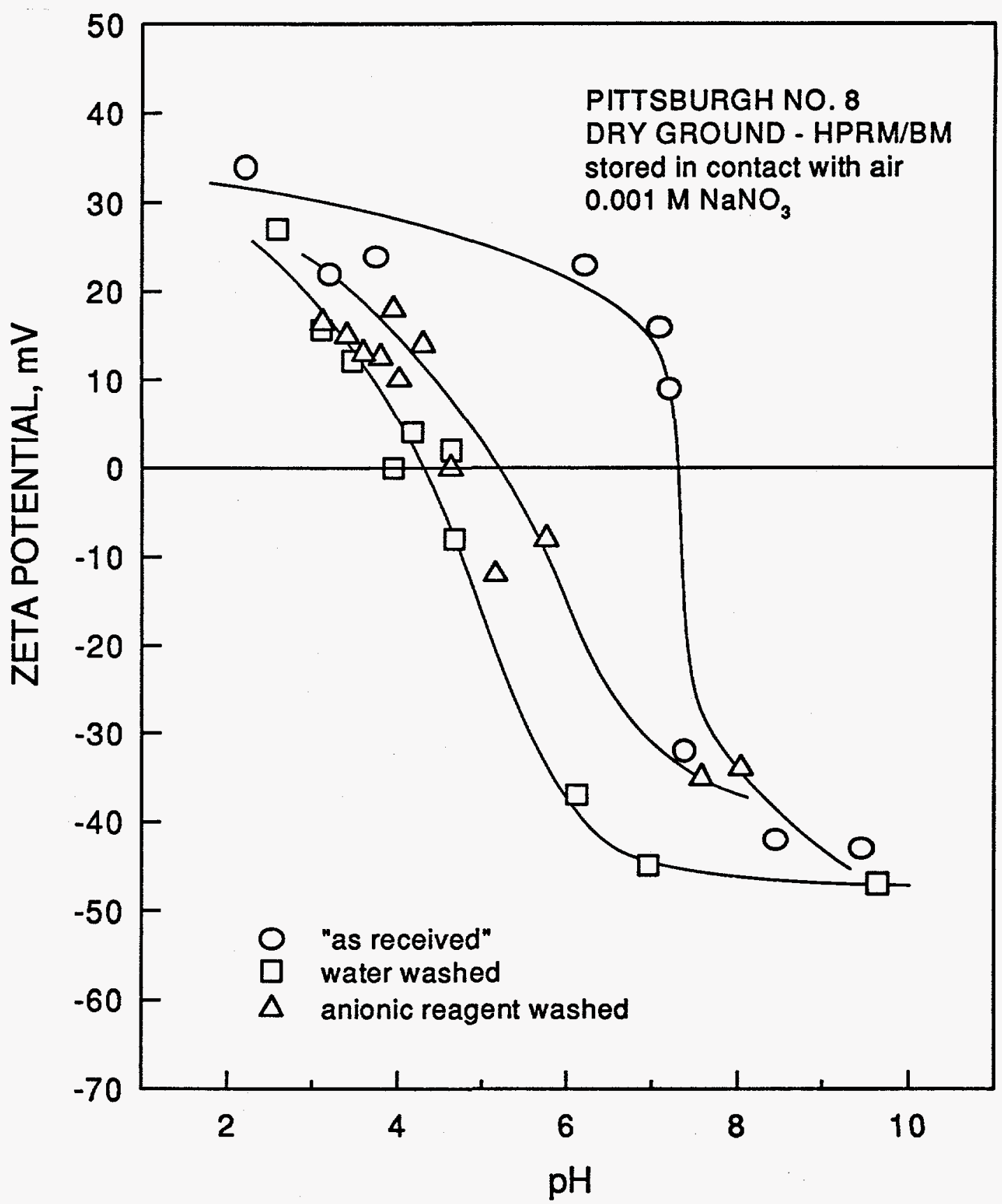

Figure 3.3. The zeta potential as a function of $\mathrm{pH}$ for unwashed, water-washed and sulfosalicylic acid-washed Pittsburgh No. 8 coal samples stored dry in contact with air for nine months. 

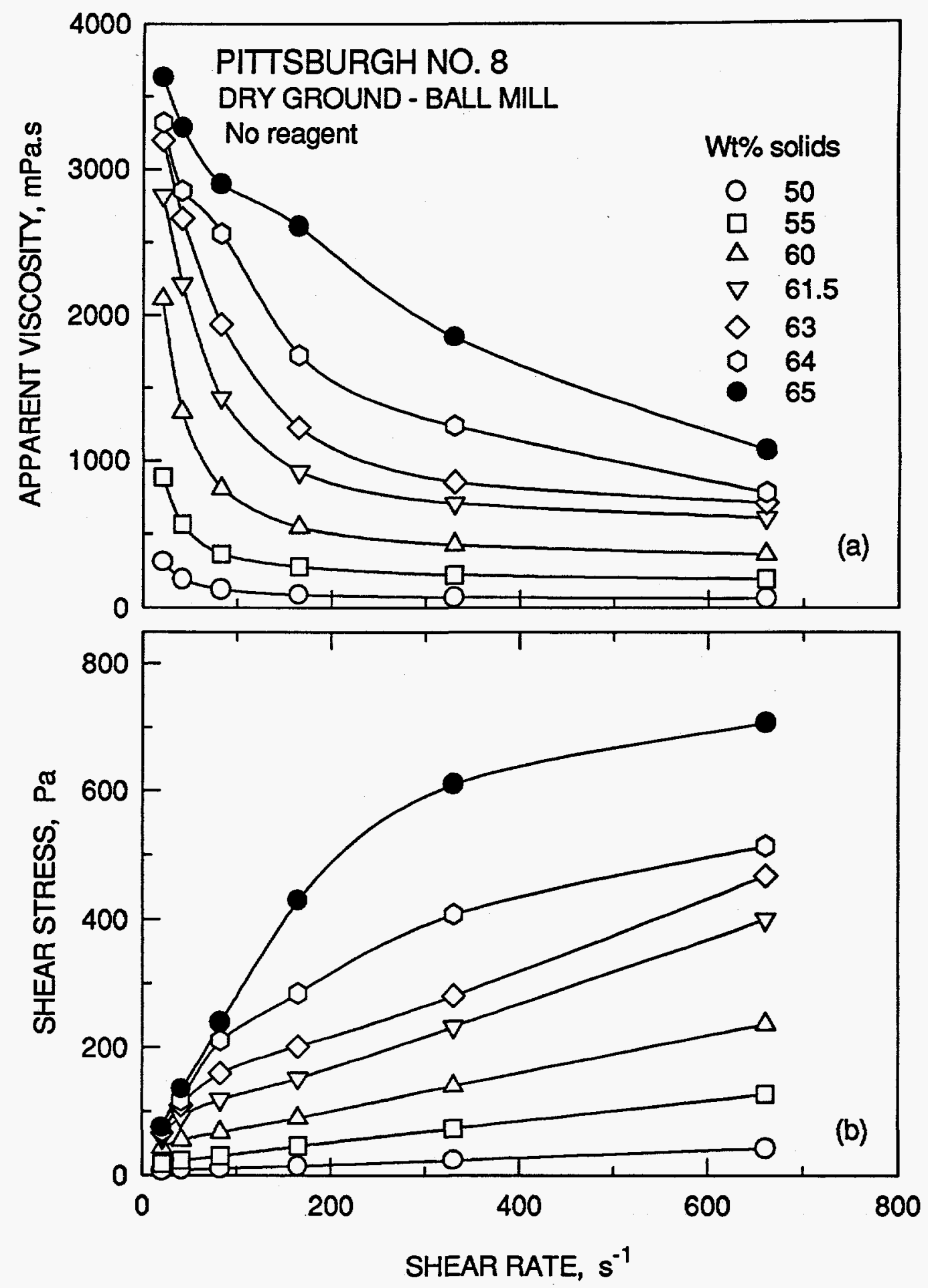

Figure 3.4. (a) The viscosity and (b) flow curves (theograms) of ball mill ground Pittsburgh No. 8 coal-water slurries as a function of shear rate, with no reagent added. 
viscosity, low sedimentation stability, and some yield stress. The systems exhibit nonNewtonian behavior and are pseudoplastic.

Analysis of the electrokinetic data also indicates that it may be possible to prepare well-dispersed coal-water slurries through the addition of suitable cationic or anionic reagents. Addition of these reagents will render coal surfaces either highly positively charged or highly negatively charged, thereby increasing the electrostatic repulsion between the particles. As will be shown later in the sub-section on the effect of chemical additives, addition of cationic and anionic reagents indeed improves the dispersion of particles in coal-water slurries.

As discussed above, there is a fairly good correlation between the electrokinetic behavior of coal particles in dilute suspensions and the rheological behavior of concentrated slurries. However, Parfitt and coworkers $[22,23]$ investigated the electrophoretic mobility of dilute and concentrated Pittsburgh No. 8 slurries and showed that the isoelectric point of concentrated slurries is located at higher pH's than the IEP of more dilute slurries. These results also suggest the presence of strongly adsorbed cationic impurities. Preliminary analysis of filtrates and supernatants from our coal-water suspensions indicated that iron is a major cationic impurity in Pittsburgh No. 8 coal (approximately $0.003 \mathrm{M}$ of iron was found in the filtrate from a 10 vol\% slurry (simple calculation shows that this could correspond to approximately $0.040 \mathrm{M}$ of iron in the $67 \mathrm{wt} \%$ (approximately $50 \mathrm{vol \% )}$ ) slurries studied in this project. Soluble cations result in a significant increase in the electrolyte concentration in concentrated suspensions and alter the electrical double layer around the particles. Overlap of double layers in concentrated suspensions might also affect the electrical interaction between particles. Therefore, zeta potential measurements at high solids loads (10 vol\%), which is somewhat more representative of a real coal-water slurry ( $50 \mathrm{vol} \%)$, were performed using the acoustophoresis technique. 


\section{Electrokinetic Behavior of Moderately Concentrated Coal-Water Slurries}

The electrokinetic behavior of coal particles in concentrated slurries may provide information on coal surface properties and on the interactions among coal particles suspended in liquid [24]. In this particular study, the feasibility of using the acoustophoresis technique to characterize coal slurries was investigated. The initial studies of Chander and coworkers [24] are analyzed and extended to the behavior of impure coal having a high concentration of cations in solution (10 vol\% Pittsburgh No. 8 coal-water slurries).

Electrokinetic sonic amplitude (ESA), which is a complex quantity that represents the magnitude and the phase of the macroscopic pressure wave induced in a suspension of charged colloidal particles when subjected to an oscillatory electric field, is a new technique for evaluating the electrokinetic behavior of concentrated suspensions [25]. The differential acceleration of the particle dipoles relative to solvent molecules produces a net momentum flux on a pair of electrodes placed in the suspension. The observed ESA signal [26] is given by

$$
\operatorname{ESA}(\omega)=\mathrm{P} / \mathrm{E}=(c)(p)(\phi)\left(\mathrm{G}_{f}\right)\left(\mu_{\mathrm{d}}\right)
$$

where:
E: amplitude of applied field gradient $(\mathrm{V} / \mathrm{m})$
c: velocity of sound in the medium $(\mathrm{m} / \mathrm{s})$
$\rho:$ density difference between particle and the continuous phase $\left(\mathrm{kg} / \mathrm{m}^{3}\right)$
$\phi:$ volume fraction of the solids (dimensionless)
$\mathrm{G}_{\mathrm{f}}$ : electrode geometrical factor (dimensionless)
$\mu_{\mathrm{d}}:$ frequency dependent electrophoretic mobility $\left(\mathrm{m}^{2} / \mathrm{sec} / \mathrm{V}\right)$

Equation 3.1 is valid in the linear regime of particle volume fraction (0.05 - 0.1 or less) and is applicable to the experiments conducted in this project. Quantitative theories for converting the dynamic electrophoretic mobility $\left(\mu_{\mathrm{d}}\right)$ in Equation 3.1 to zeta potential from the ESA signal for polydispersed systems have been developed [27]. Knowledge of the particle size distribution and density is required to make this conversion: the higher the particle density (actually density difference between particles and solvent), the stronger is the resulting signal. 
This creates problems in the case of coal because its density $\left(1.32 \mathrm{~g} / \mathrm{cm}^{3}\right)$ is only slightly higher than the density of water $\left(1.0 \mathrm{~g} / \mathrm{cm}^{3}\right)$ and the observed signal is low.

The presence of ions in solution generates an electroacoustic signal. In such situations where the solid signal is low, the ion background signal should be subtracted from the actual ESA readings. Since ESA is a vector (it has a magnitude and a phase angle) the subtraction process has to be done vectorially. The method of Desai and Hayes [28] was used to calculate the "true" ESA signal from coal particles:

$$
\begin{aligned}
& \text { ESA }_{\text {true }} \cos \beta=\left[\text { ESA }_{\text {measured }} \cos \Theta\right]-\left[\mathrm{ESA}_{\text {background }} \cos \Phi\right] \\
& \mathrm{ESA}_{\text {true }} \sin \beta=\left[\mathrm{ESA}_{\text {measured }} \sin \Theta\right]-\left[\mathrm{ESA}_{\text {background }} \sin \Phi\right]
\end{aligned}
$$

where $\beta$ is the phase angle of the suspended solid, $\Phi$ is the phase angle of the background electrolyte + any electrolyte from the solid impurities leached out during experiment and $\Theta$ is the measured phase angle for the total sample (solid + electrolyte).

In the case of pure coal analyzed by Chander and coworkers [24], the change in $\mathrm{pH}$ did not influence the ESA signal significantly. In the case of our Pittsburgh No.8 coal sample, $\mathrm{pH}$ significantly influenced the background ESA signal due to the presence of a high amount of iron $(0.003 \mathrm{M})$. Consequently, we had to read the magnitude and the phase angle of measured and electrolyte signals for every $\mathrm{pH}$ and calculate the "true" ESA signal for every titration point, unlike Chander and coworkers who just had to subtract indifferent electrolyte signal (coming from $\mathrm{KNO}_{3}$ which they added to keep ionic strength constant).

There is an additional problem with coal prepared for CWS in that the ground coal has a broad particle size distribution. In order to correctly calculate zeta potentials from ESA data, an inertia factor has to be calculated to correct for the effective inertial terms. This factor can be calculated by summing inertia factors for different size intervals multiplied by their volume fractions. In order to obtain reliable value of the zeta potential, the particle size distribution must be measured accurately. For this purpose, we used a Laser Microtrac Particle Sizer, which is fairly reliable for particle sizes between 2 and 150 microns. 


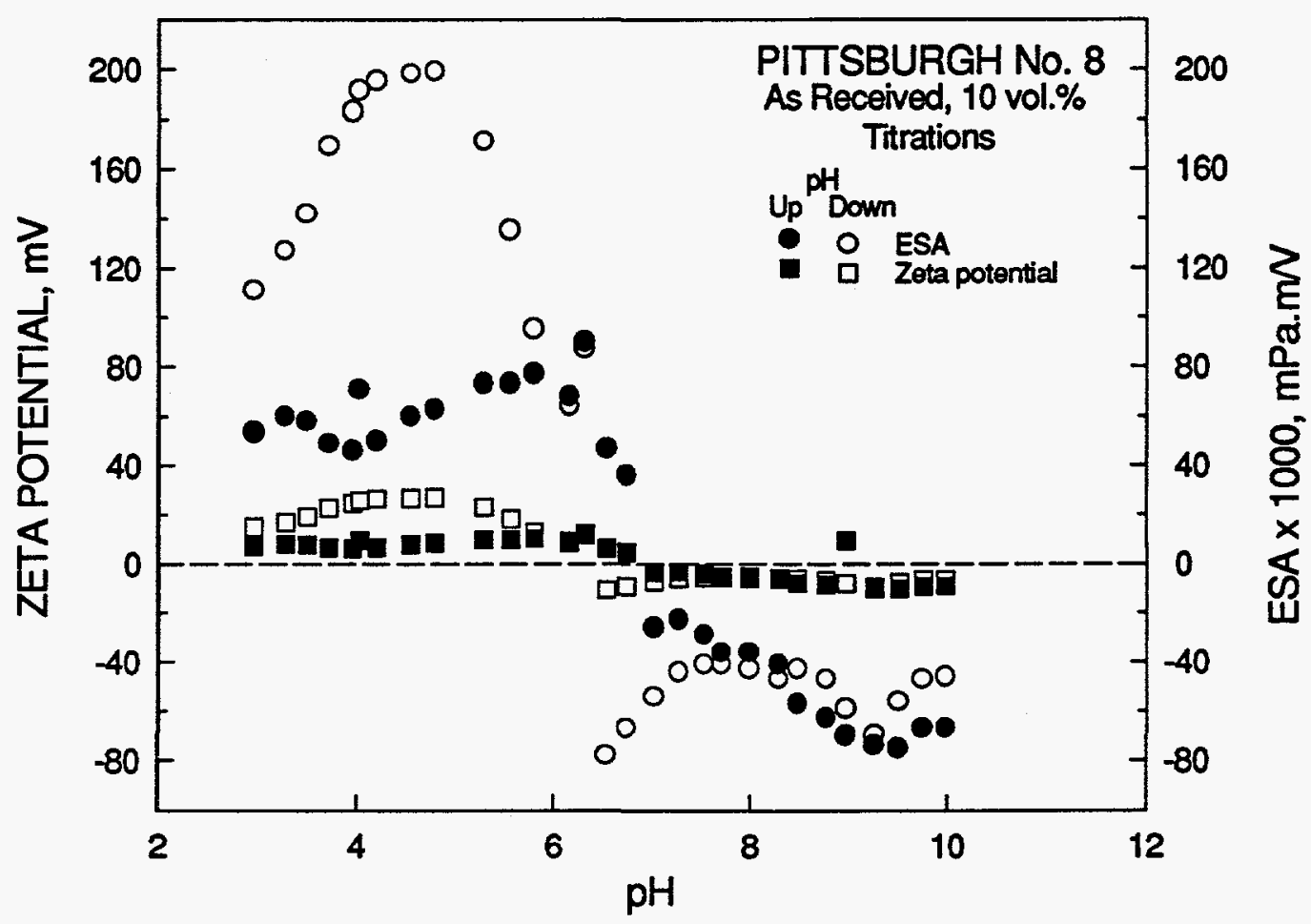

Figure 3.5. The zeta potential and true ESA values measured by acoustophoresis as a function of $\mathrm{pH}$ (up and down titrations) for 10 volume percent coal-water slurry prepared using unwashed ball mill product stored dry under agron.

Figure 3.5 presents zeta potentials of 10 vol\% Pittsburgh No. 8 slurries prepared from coal stored under argon. This coal sample was prepared from "as-received" ground materials without washing. The IEP of that coal sample was found to occur at $\mathrm{pH} \mathrm{7,} \mathrm{which} \mathrm{coincides}$ well with the traditional measurements, but the measured zeta potentials were significantly lower (only a few milivolts). This is characteristic of strongly flocculated systems. Rheology measurements of coal samples in the absence of any dispersion reagent indeed indicated that the system was strongly flocculated.

The effect of filtration and resuspension of the same coal samples is illustrated by the plots given in Figure 3.6. Once again, zeta potentials measured were very low (a few milivolts) but the IEP shifted to $\mathrm{pH} 6$. We always analyzed the up curves, that is titration of the slurries was carried out from the acidic $\mathrm{pH}$ to basic $\mathrm{pH}$ region. Down curves were done only to show that some precipitation takes place as the coal is subjected to high $\mathrm{pH}$. 


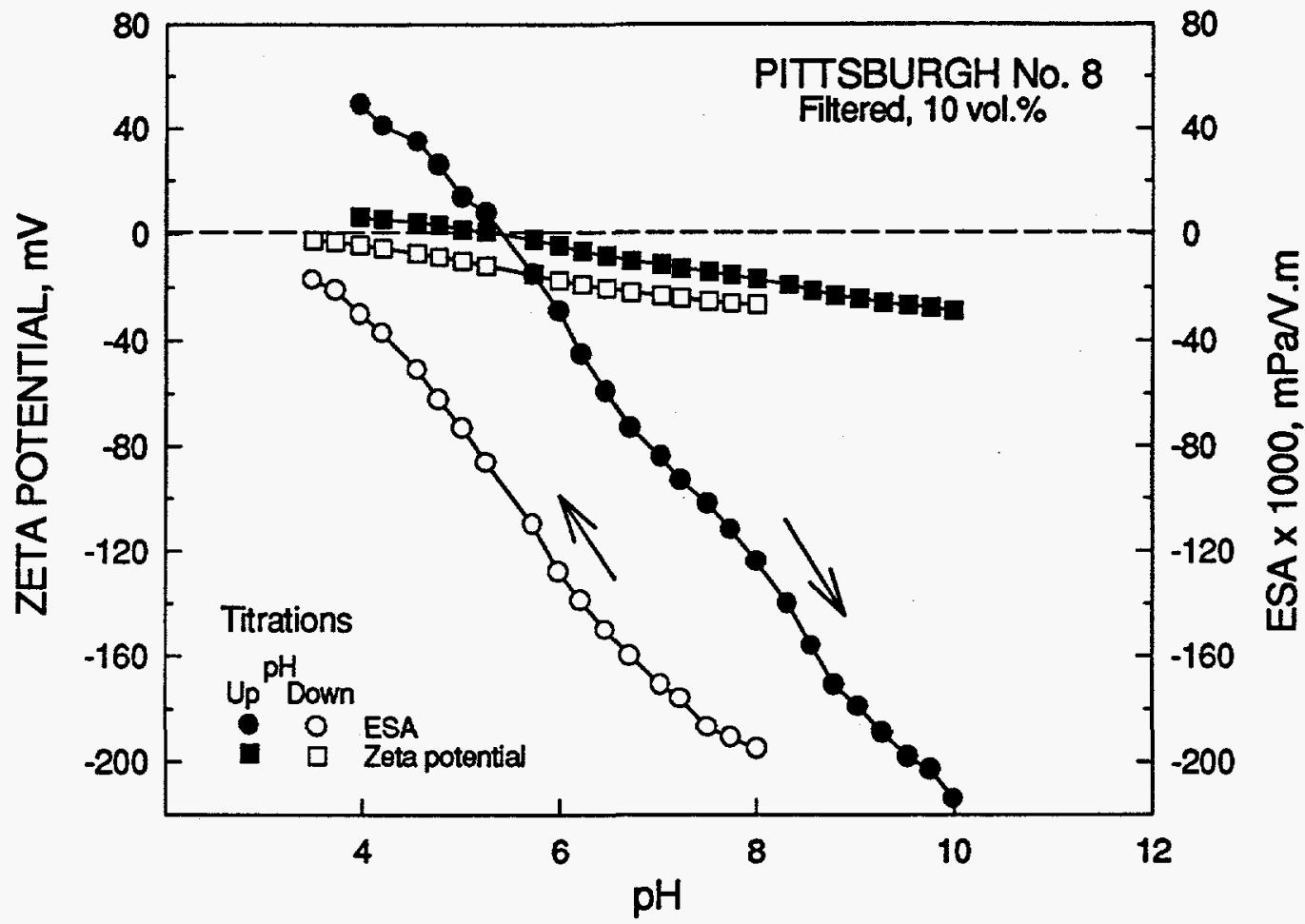

Figure 3.6. The zeta potential and true ESA values measured by acoustophoresis as a function of $\mathrm{pH}$ (up and down titrations) for 10 volume percent coal-water slurry prepared using a filtered and resuspended ball mill product stored dry under agron.

Figure 3.7 shows the effect of multiple washing of the coal with distilled water on the measured zeta potential and the IEP. The IEP of thoroughly washed coal was found to be at $\mathrm{pH} 5.7$, with zeta potentials ranging from +20 to $-12 \mathrm{mV}$. Once again, the measured zeta potentials of moderately concentrated CWS were significantly lower than for the highly diluted samples. This indicates that though zeta potential measurements of slurries diluted 10000 times can help identify whether specifically adsorbed anions or cations cause the strong flocculation of the CWS, but such measurements cannot clearly correlate with the stability and viscosity of the slurry.

In the end, only apparent viscosity measurements can help us understand the stability and flocculation/stabilization behavior of coal-water slurries. However, zeta potential measurements can help in identifying impurities responsible for the aging effect and in 


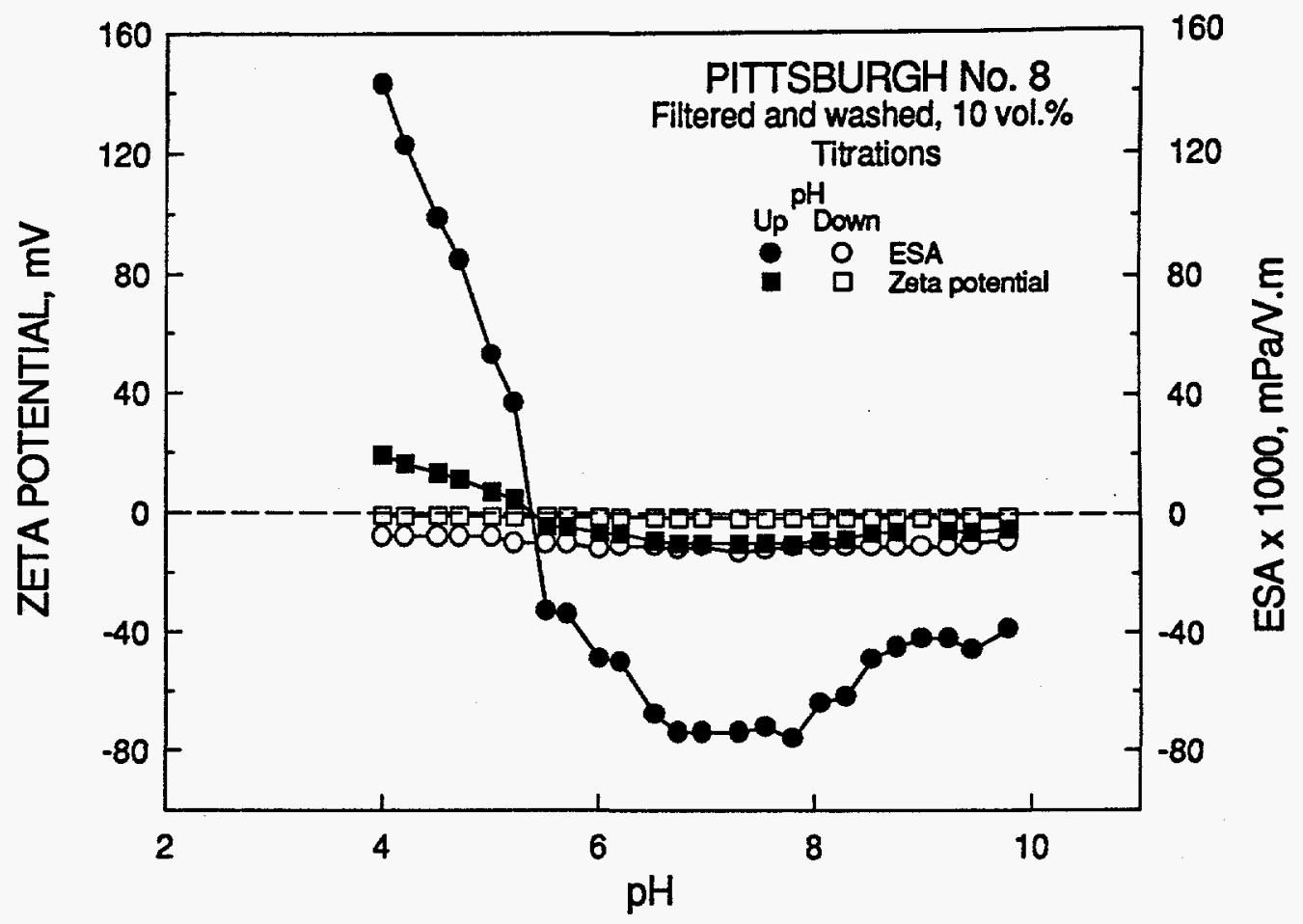

Figure 3.7. The zeta potential and true ESA values measured by acoustophoresis as a function of $\mathrm{pH}$ (up and down titrations) for 10 volume percent coal-water slurry prepared using a filtered and thoroughly water-washed ball mill product stored dry under agron.

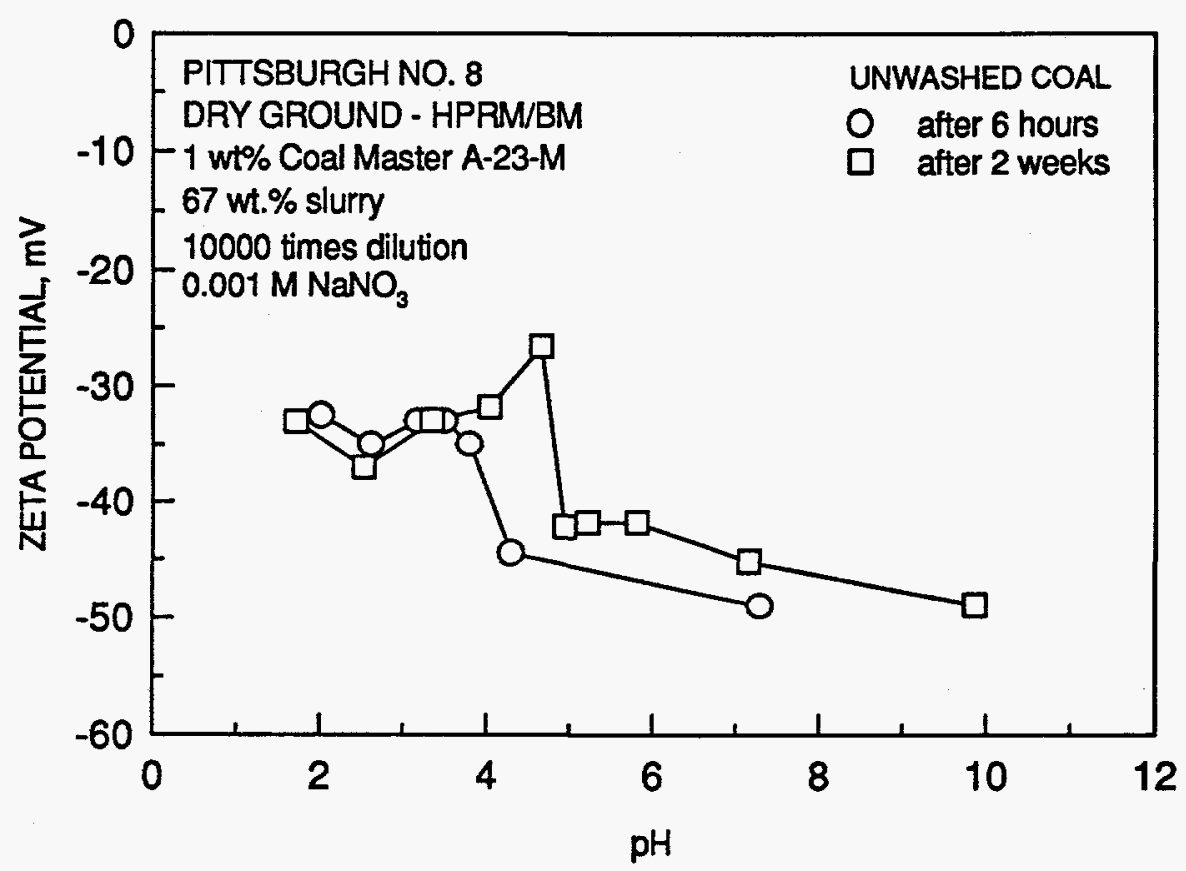

Figure 3.8. The zeta potential as a function of $\mathrm{pH}$ for diluted coal-water slurry prepared using a water-washed ball mill product and $1.0 \mathrm{wt}$. percent CoalMaster A-23M and equlibrated for various time periods. 
understanding the mechanism of their action. Traditional zeta potential measurements carried out on slurry prepared using unwashed "as-received" coal and aged for different lengths of time is a good example. Figure 3.8 shows the measured zeta potential for the highly diluted slurry (10,000 times) after 6 hours and 2 weeks. No drastic change in zeta potential was observed. On the other hand, slurry viscosity increased significantly after two weeks.

\section{EFFECT OF CHEMICAL ADDITIVES ON THE RHEOLOGY OF COAL-WATER SLURRIES}

The surface characteristics of coal particles play an important role in determining the particle-particle interaction in concentrated coal-water slurries and hence their theolgical behavior. Particluate interactions could be lowered by either increasing the electrostatic repulsion between particles by addition of cationic or anionic reagents, or increasing steric hindrance by addition of nonionic surfactants with high molecular weights, or both by addition of polyelectrolytes. In this project, we have investigated the effect of addition of the following reagents on the rheology of coal-water slurries prepared with fines produced by ball mill grinding of coal:

1) Triton $\mathrm{X}$ series of nonionic surfactants $\left[\mathrm{C}_{8} \mathrm{H}_{17}-\mathrm{C}_{6} \mathrm{H}_{4}-\left(\mathrm{O}-\mathrm{CH}_{2}-\mathrm{CH}_{2}\right)_{n}-\mathrm{OH}\right.$, with different number of ethoxy groups: Triton X-100 ( $n=9-10)$, Triton X-102 $(n=12-13)$, Triton X-165 ( $n=16)$, Triton X-305 ( $n=30)$, and Triton X-405 $(n=40)$.

2) an anionic surfactant, 2-isothiouronium-ethane phosphonic acid (2-TEPA), with molecular weight of 185 , and

3) a cationic surfactant, 2-trimethylammonium-ethane isothiouronium dichloride (TMAE), with molecular weight of 235 ,

4) a polyelectrolyte, sulfonated napthalene condensate (CoalMaster A-23-M), with molecular weight of about 3600 .

Slurries of Pittsburgh No. 8 coal that had been dry ground to 90 percent minus 200 mesh were prepared with various dosages of chemical additives and conditioned at $20^{\circ} \mathrm{C}$ in a 
shaker for 16 hours. Then the shear stress of each slurry was measured as a function of shear rate using the Haake RV-12 viscometer with the MV-DIN sensor system.

Figure 3.9 shows the flow curves for Pittsburgh No. 8 coal-water slurries at different additions of Triton X-165. It can be seen from the results given in Figure 3.9 that the shear stress of the slurries generally decreases with increasing Triton X-165 additions at a fixed shear rate, except at $0.253 \mathrm{wt} \%$ addition. Furthermore, the coal slurries behave as pseudoplastic fluids with a yield stress until the reagent addition is higher than $0.76 \mathrm{wt} \%$. At an addition of $1.01 \mathrm{wt} \%$ Triton $\mathrm{X}-165$, the rheological behavior of the slurries tends to approach that of a Bingham type of fluid.

Figure 3.10 presents the viscosity of the slurry as a function of the Triton X-165 addition at three different shear rates. The results given in this figure show that the viscosity of the slurries decreases at a Triton X-165 addition of $0.25 \mathrm{wt} \%$ and then increases slightly at

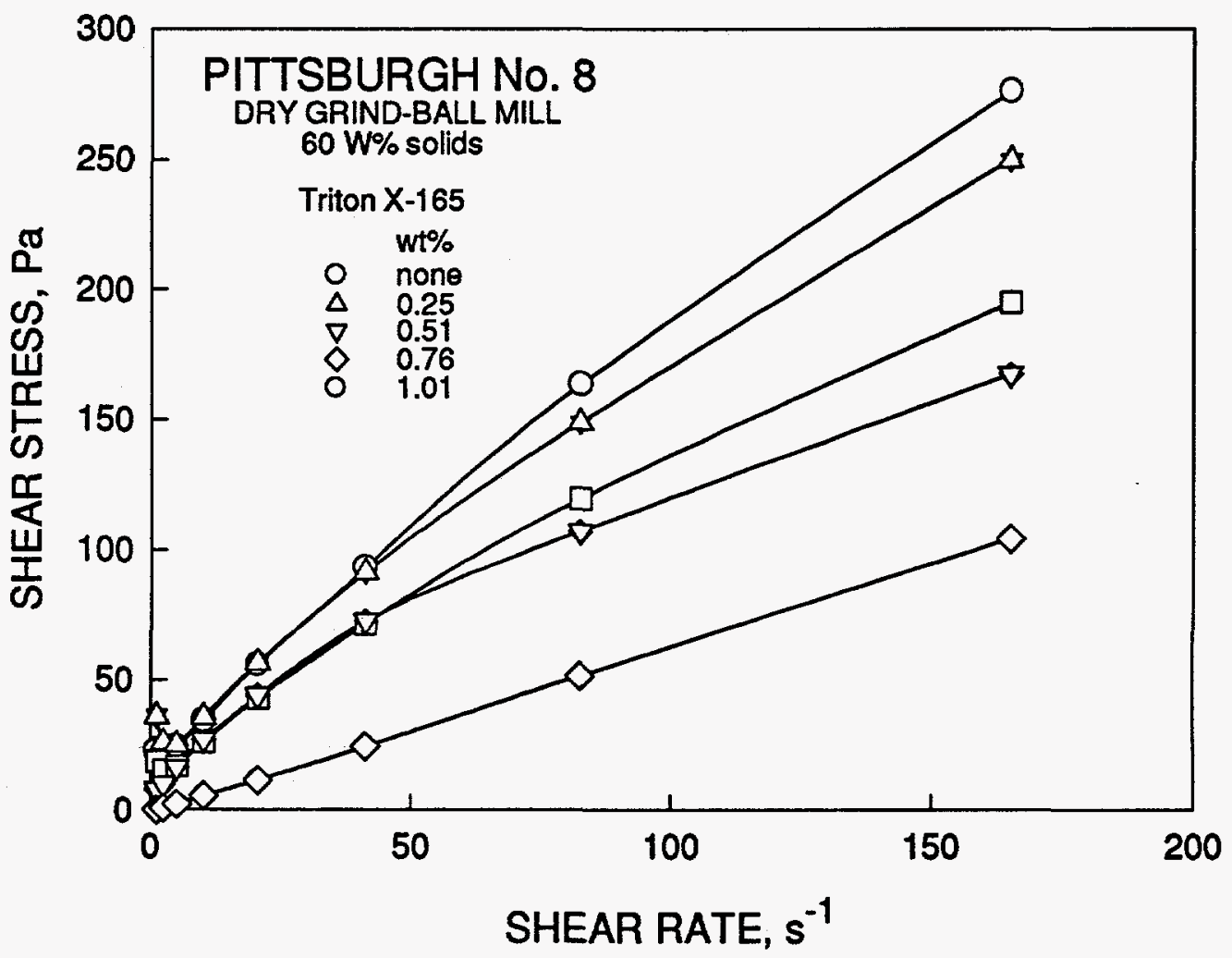

Figure 3.9. Shear stress as a function of shear rate for Pittsburgh No. 8 coal-water slurries containing $60 \mathrm{wt} \%$ solids in presence of various Triton X-165 additions. 


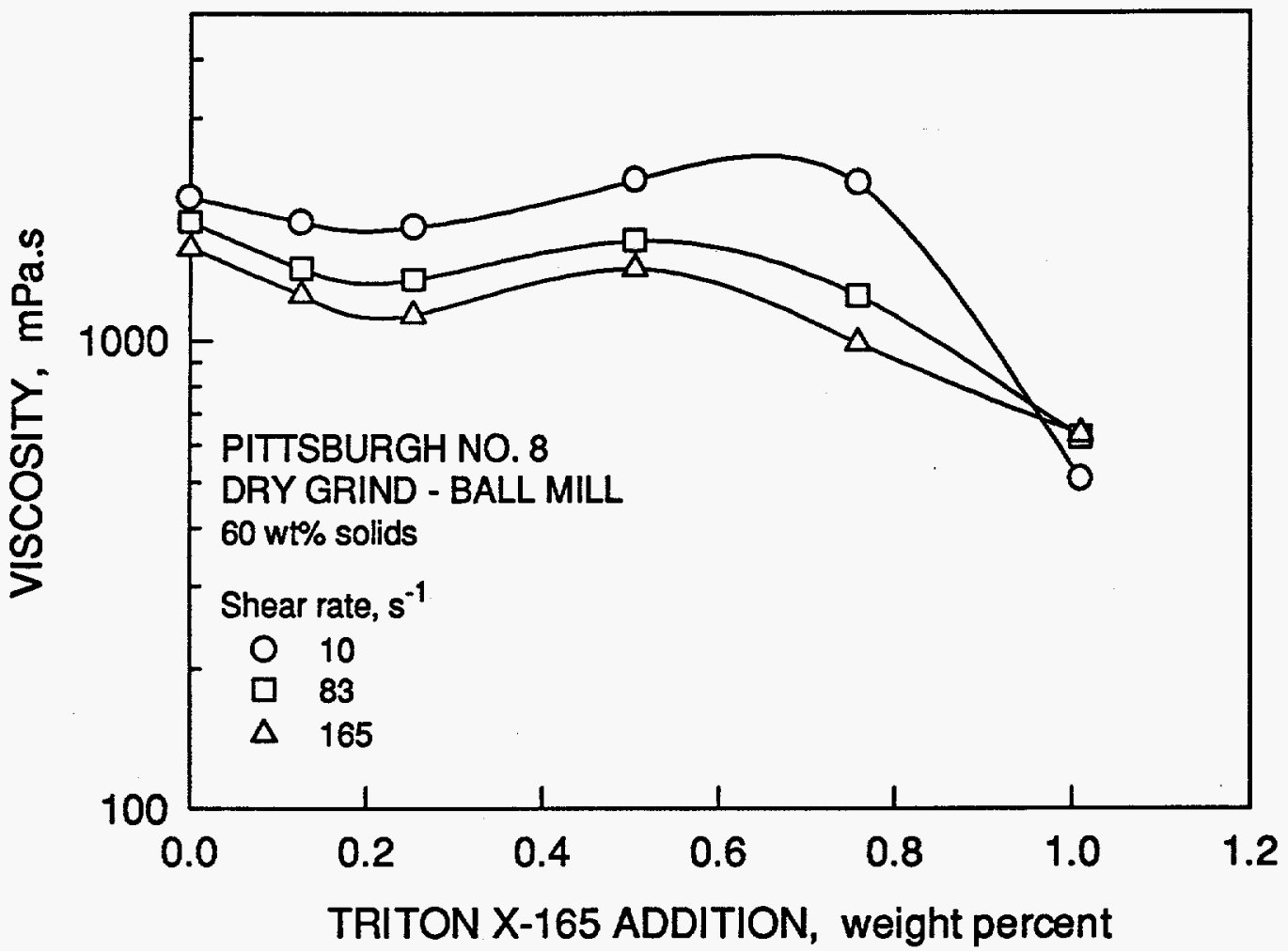

Figure 3.10. Effect of Triton X-165 addition on the viscosity of Pittsburgh No. 8 coal-water slurries containing $60 \mathrm{wt} \%$ solids at three different shear rates.

$0.50 \mathrm{wt} \%$ addition. The viscosity of the slurries decreases further at higher reagent additions $0.76 \mathrm{wt} \%$ and $1.01 \mathrm{wt} \%$. This behavior could be related to the balance between the decrease in particle-particle attraction (decrease viscosity) and the increase in the effective volume radii of the particles (increase viscosity) as the adsorption of the hydrophilic ethylene oxide groups increases $[29,30]$. Furthermore, increasing the shear rate decreases slightly the viscosity of the slurries until the reagent addition reaches $1.0 \mathrm{wt} \%$, At which the viscosity is independent of shear rate.

In the case of Triton X-100, X-102, X-305 and X-405, similar behavior was observed. For example, Figure 3.11 presents the apparent viscosity as a function of the addition of Triton X-100, X-165 and X-405 reagents. Clearly, an increase in reagent addition for the Triton $\mathrm{X}$ series follows the general behavior described for Triton $\mathrm{X}-165$. The effect of the number of ethylene oxide groups in a surfactant molecule on the viscosity is given in 


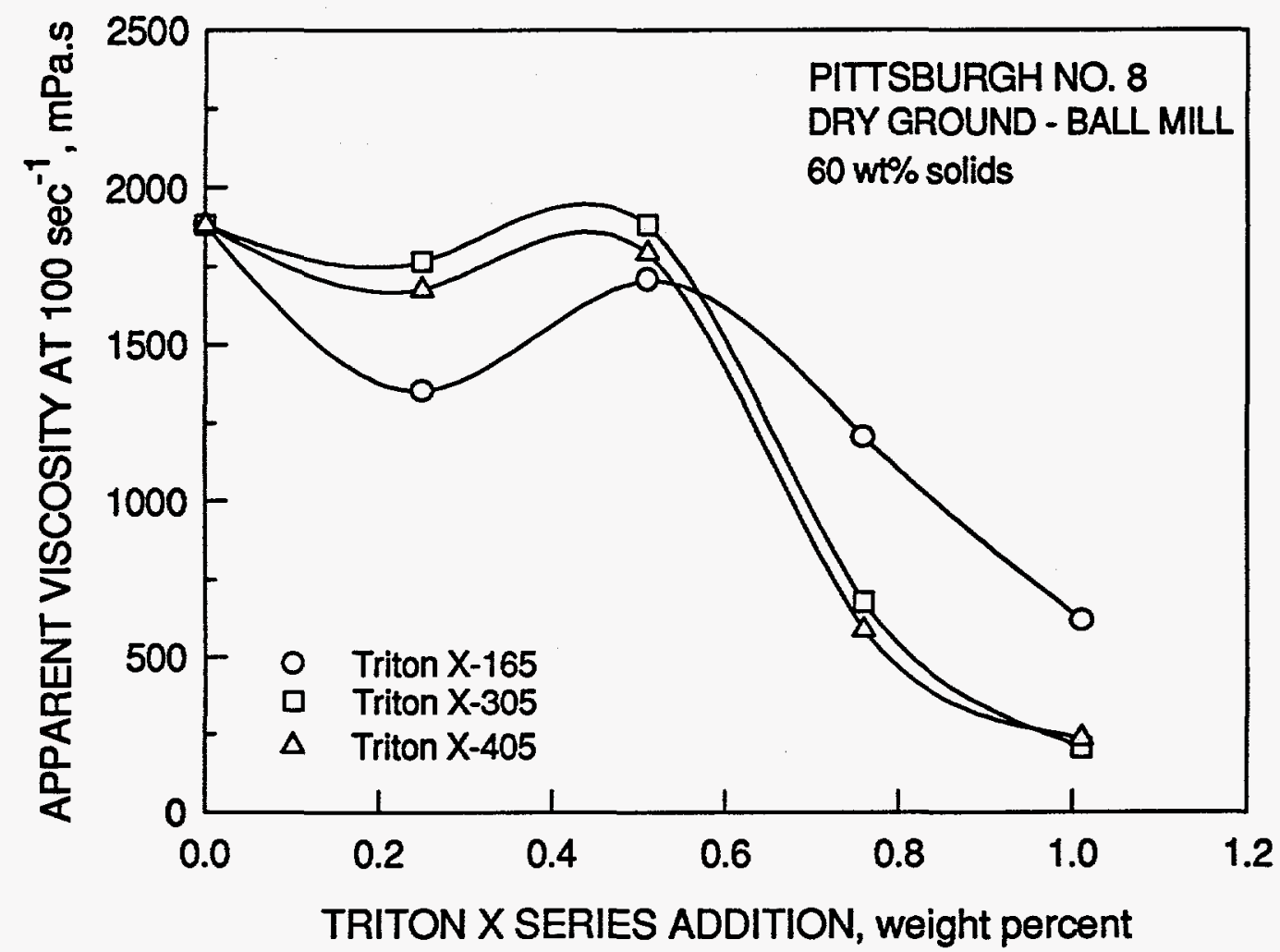

Figure 3.11. Effect of addition of different Tritons on the viscosity of Pittsburgh No. 8 coal-water slurries containing $60 \mathrm{wt} \%$ solids at a shear rate of $100 \mathrm{~s}^{-1}$.

Figure 3.12. These plots show that at low reagent addition $(0.13 \mathrm{wt} \%$ and $0.5 \mathrm{wt} \%)$ the effect of the number of ethylene oxide groups is insignificant. However, at high reagent addition (1.0 wt\%), the viscosity of the slurries decreases significantly with the increase in the number of ethylene oxide groups in the surfactant molecule. This is due to the more hydrophilic nature of molecules having a larger number of ethylene oxide groups as compared to those with a smaller number.

2-TEPA, an anionic hydrophilic reagent with a short hydrocarbon chain, belongs to a family of reagents that contains sulfur and nitrogen in its molecule. Even though this reagent has a short hydrocarbon chain in comparison with the Triton $\mathrm{X}$ series of reagents, the hydrophilic 2-TEPA was selected to evaluate whether reagents having functional groups of high electronegativity could disperse the system better. 


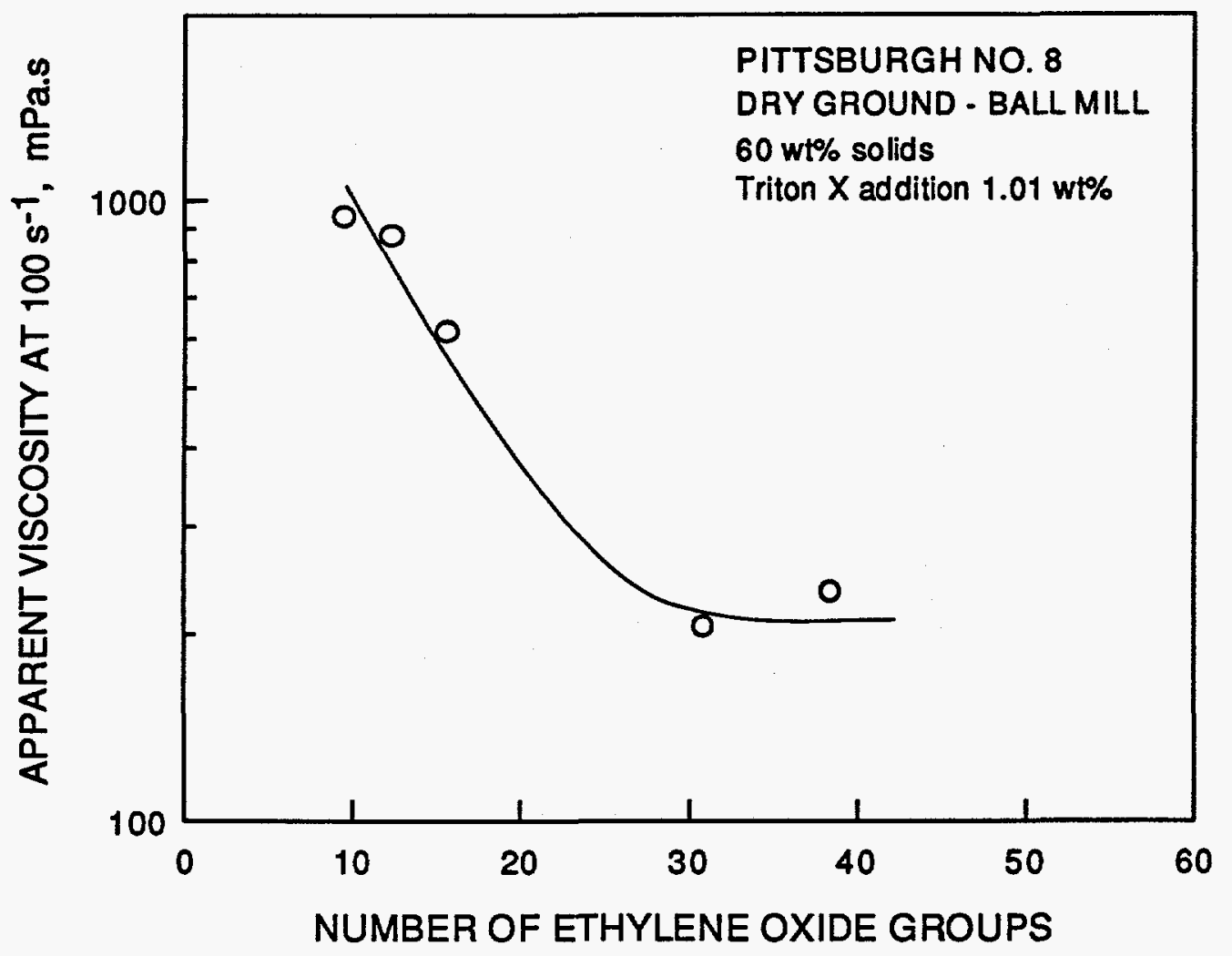

Figure 3.12. Viscosity of Pittsburgh No. 8 coal-water slurries as a function of the number of ethylene oxide groups in the surfactant molecule at a shear rate of $100 \mathrm{~s}^{-1}$.

Figure 3.13, which presents the effect of 2-TEPA concentration on the shear stress of Pittsburgh No. 8 coal-water slurries as a function of the shear rate, shows that the shear stress decreases as the reagent addition increases and that the coal-water slurries maintain pseudoplastic behavior with a yield stress for all reagent additions. The effect of 2-TEPA on the viscosity is shown in Figure 3.14. As expected, this figure shows that the viscosity is decreased with an increase in the reagent dosage and the shear rate. Figure 3.15 presents the measured viscosity as a function of the 2-TEPA addition for four different shear rates. Similar to the case for nonionic surfactants, the viscosity decreases as the concentration isincreased. This reagent seems to decrease the viscosity of the slurries by adsorbing on the surface of coal particles, which increases the electrical repulsive forces and hydrophilicity of the coal particles. At $0.5 \mathrm{wt} \%$ addition of 2-TEPA, a small increase in viscosity is observed. This 


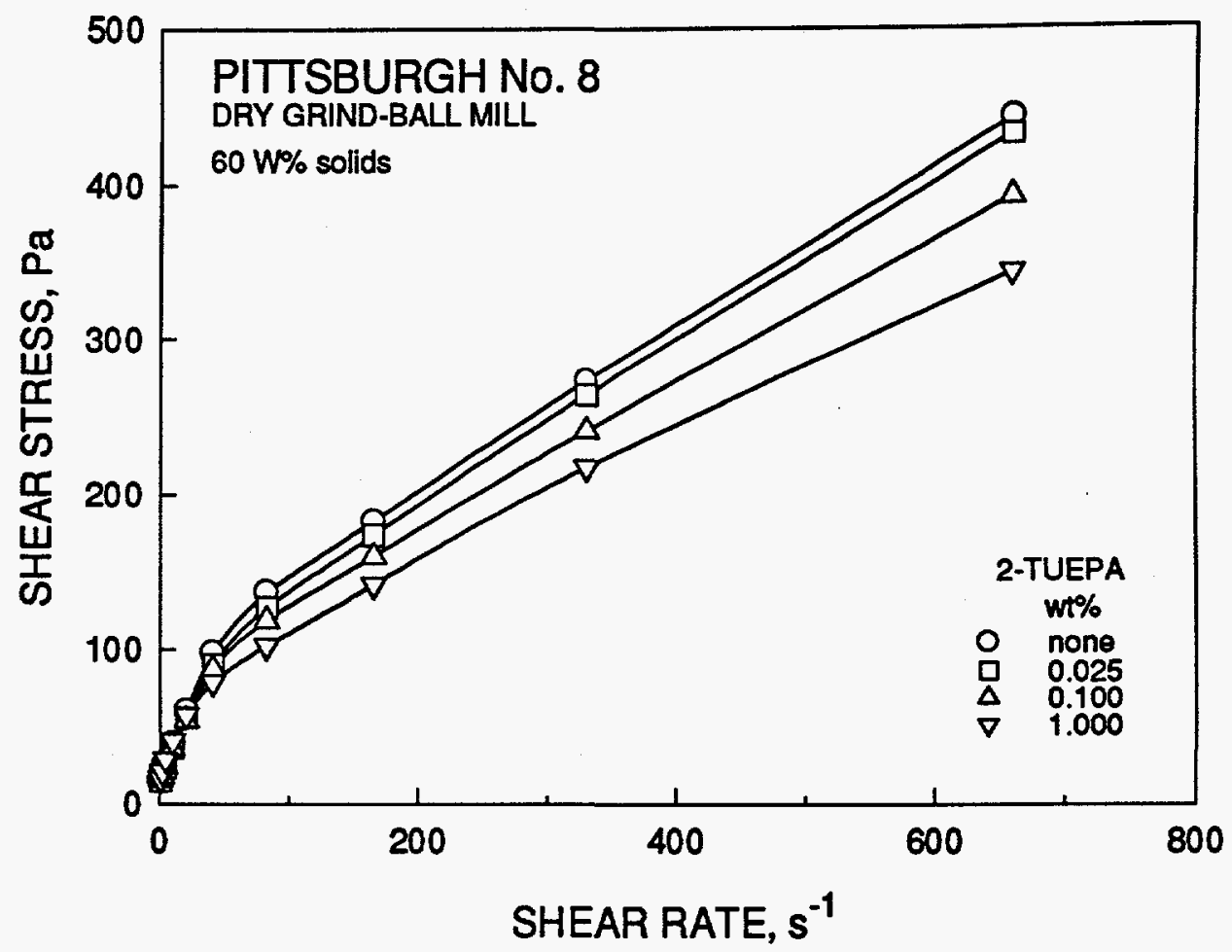

Figure 3.13. Flow curves (rheograms) of Pittsburgh No. 8 coal-water slurries having 60 wt\% solids at different 2-TEPA additions.

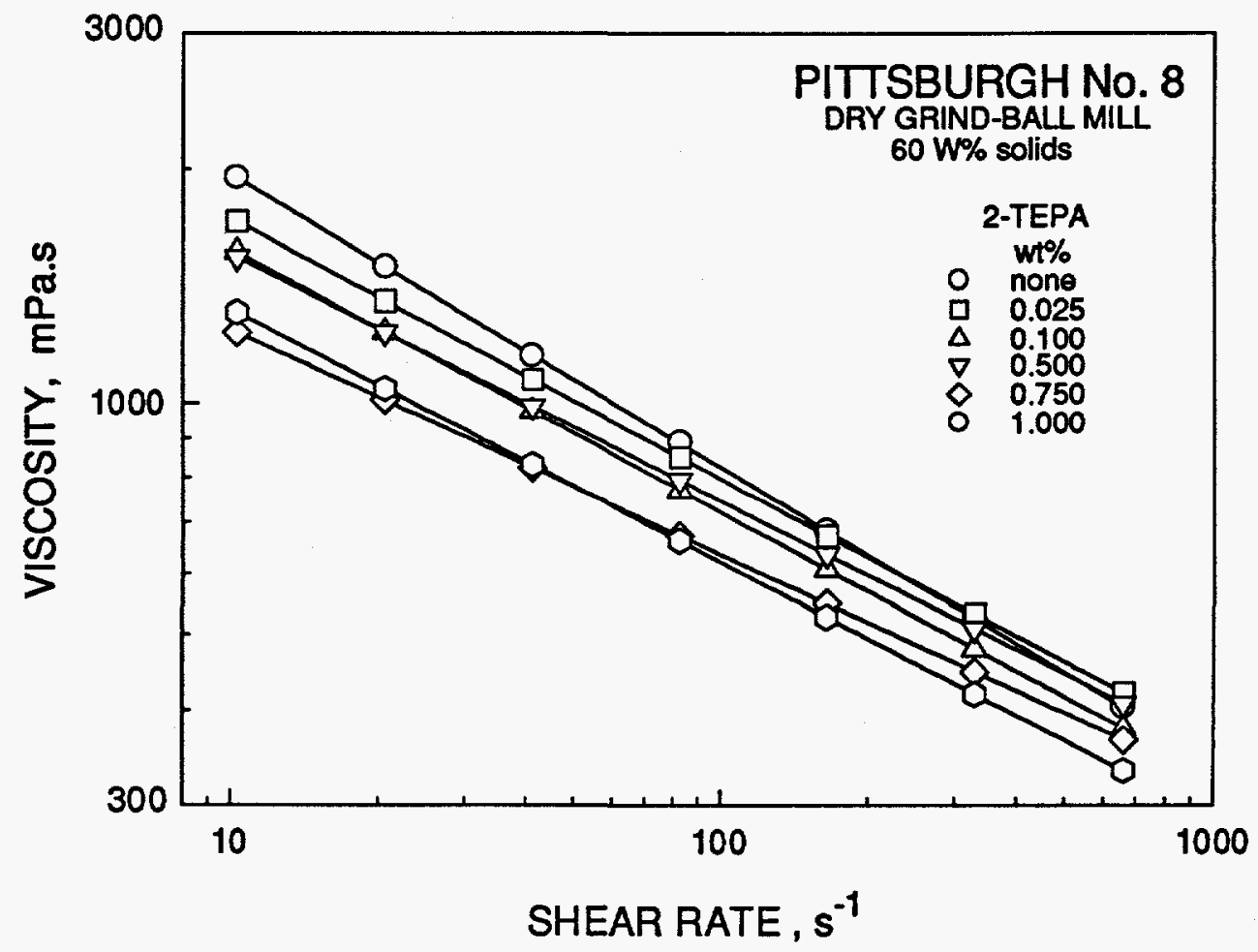

Figure 3.14. Viscosity of Pittsburgh No. 8 coal-water slurries having $60 \mathrm{wt} \%$ solids as a function of shear rate at different 2-TEPA additions. 


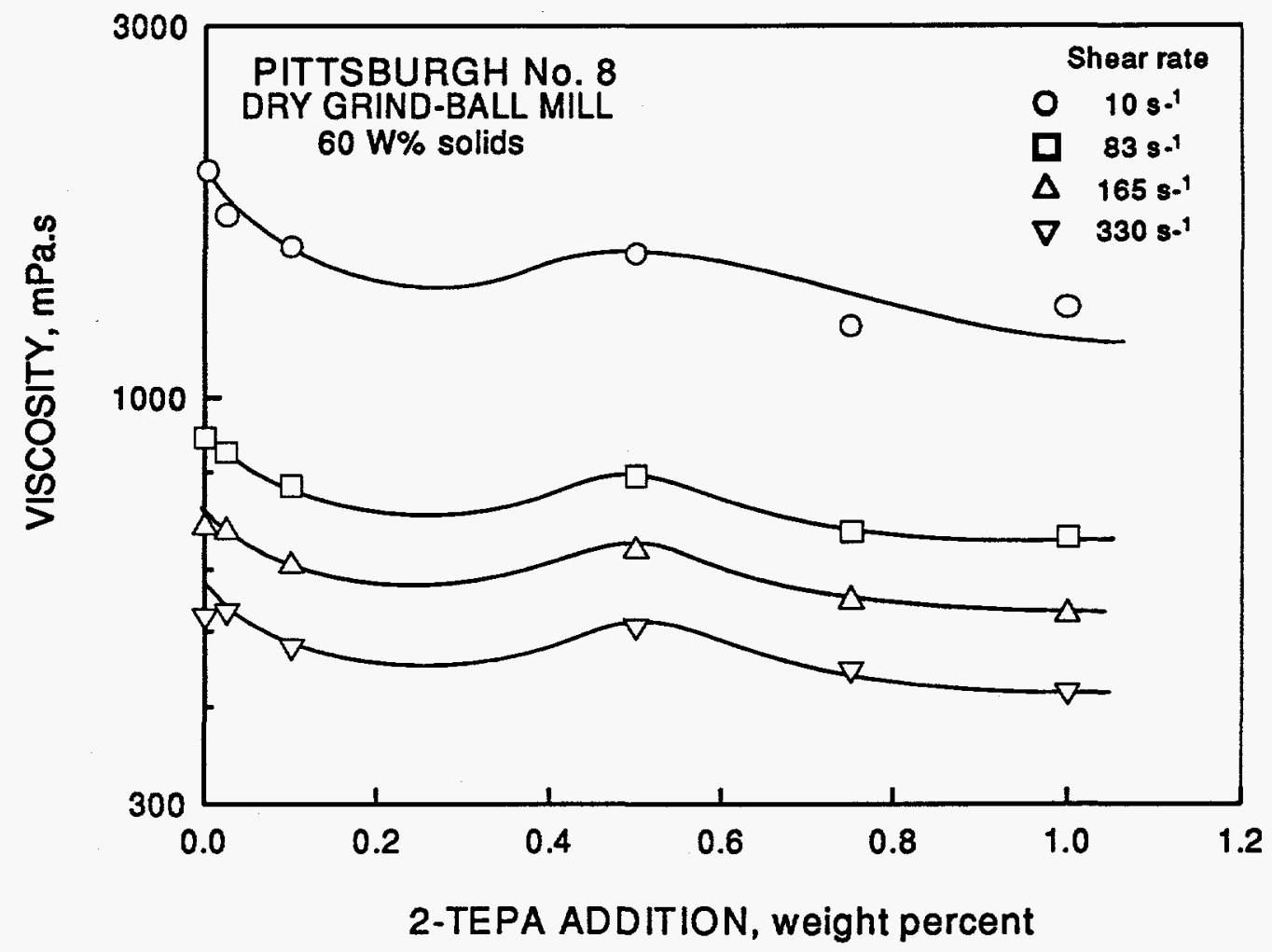

Figure 3.15. Viscosity of Pittsburgh No. 8 coal-water slurries as a function of 2-TEPA addition at four different shear rates.

could be related to the increase in the effective radii of the particles due to the adsorption of this hydrophilic compound on the coal surface, as discussed previously.

To evaluate the effect of TMAE on the rheological behavior of Pittsburgh No. 8 coalwater slurries, experiments were carried out with slurries containing $60 \mathrm{wt} \%$ coal that had been ground to $95 \%$ minus 200 mesh ( 74 microns). TMAE is a reagent of the same family as 2-TEPA, containing sulfur and nitrogen functional groups in its molecule. TMAE, which is a hydrophilic cationic reagent of a short hydrocarbon chain as compared to the Triton $\mathrm{X}$ series of reagents, appears to strongly adsorb onto the surface of coal through its positively charged functional group. Since TMAE is a cationic surfactant, it can adsorb onto the negatively charged surface of the coal particles, causing a reduction in the magnitude of the Stern layer potential (or zeta potential). 


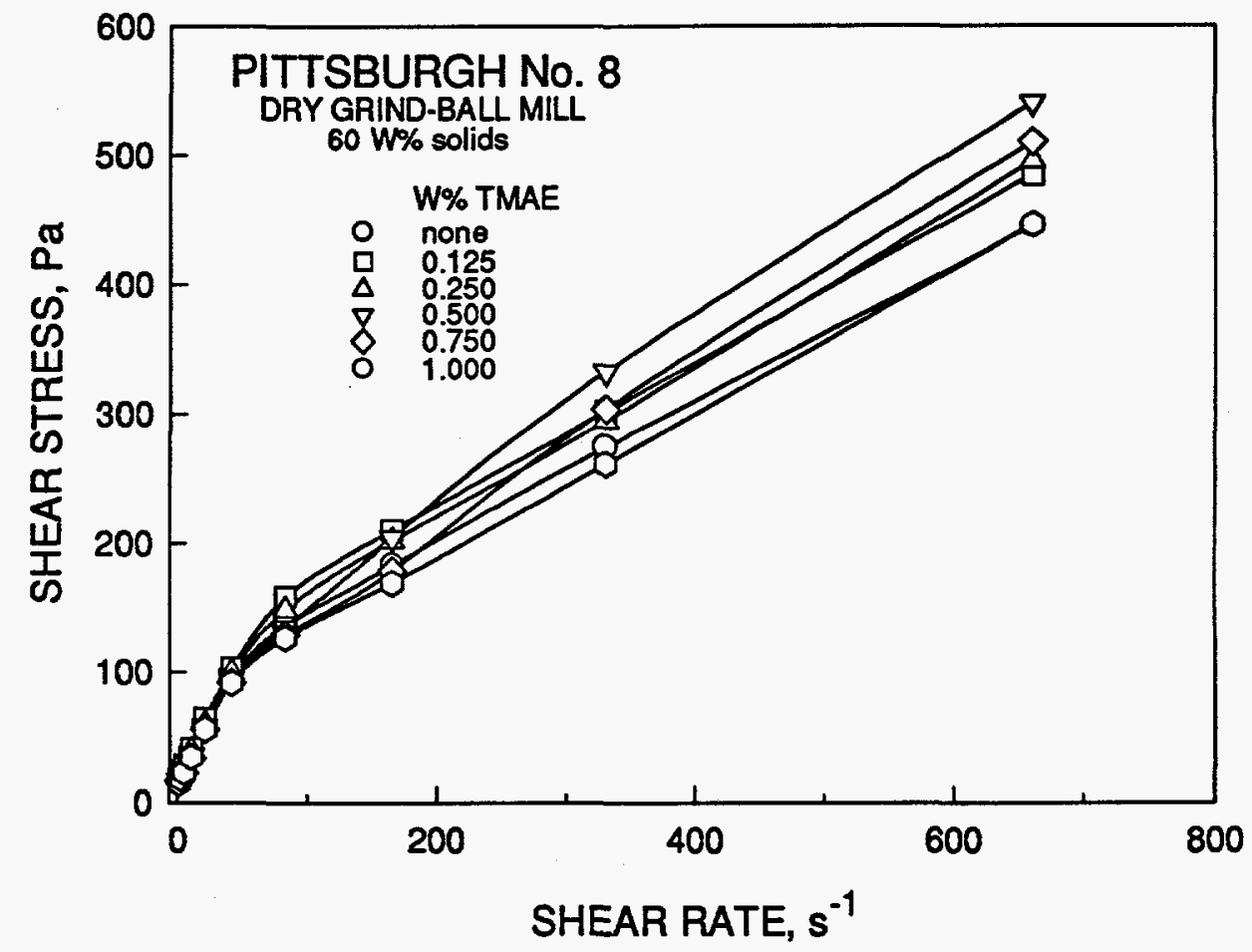

Figure 3.16. Flow curves (mheograms) of Pittsburgh No. 8 coal-water slurries having 60 wt $\%$ solids at different TMAE additions.

Figure 3.16 presents the effect of TMAE addition on the flow curve of coal-water slurries made from Pittsburgh No. 8 coal. In this system, the rheological behavior of the system exhibits a pseudoplastic behavior with yield stress for all reagent dosages tested. The plots show that the reagent initially increases the shear stress for a given shear rate up to an addition of $0.5 \mathrm{wt} \%$, indicating the flocculation of the particles. Further increase in the amount of reagent decreases the shear stress for a given shear rate to values obtained in the absence of this reagent. This decrease in the shear stress must be related to reagent adsorption at the coal-water interface. Maximum flocculation would occur when at reagent additions that bring about reversal of the zeta potential, and redispersion would occur when the double layer at the coal surface becomes recharged. Thus, TMAE can flocculate the particles at low surface coverage and weakly flocculate or redisperse them at high surface coverage as the net charge builds up on the positive side. Another possible contribution to the 


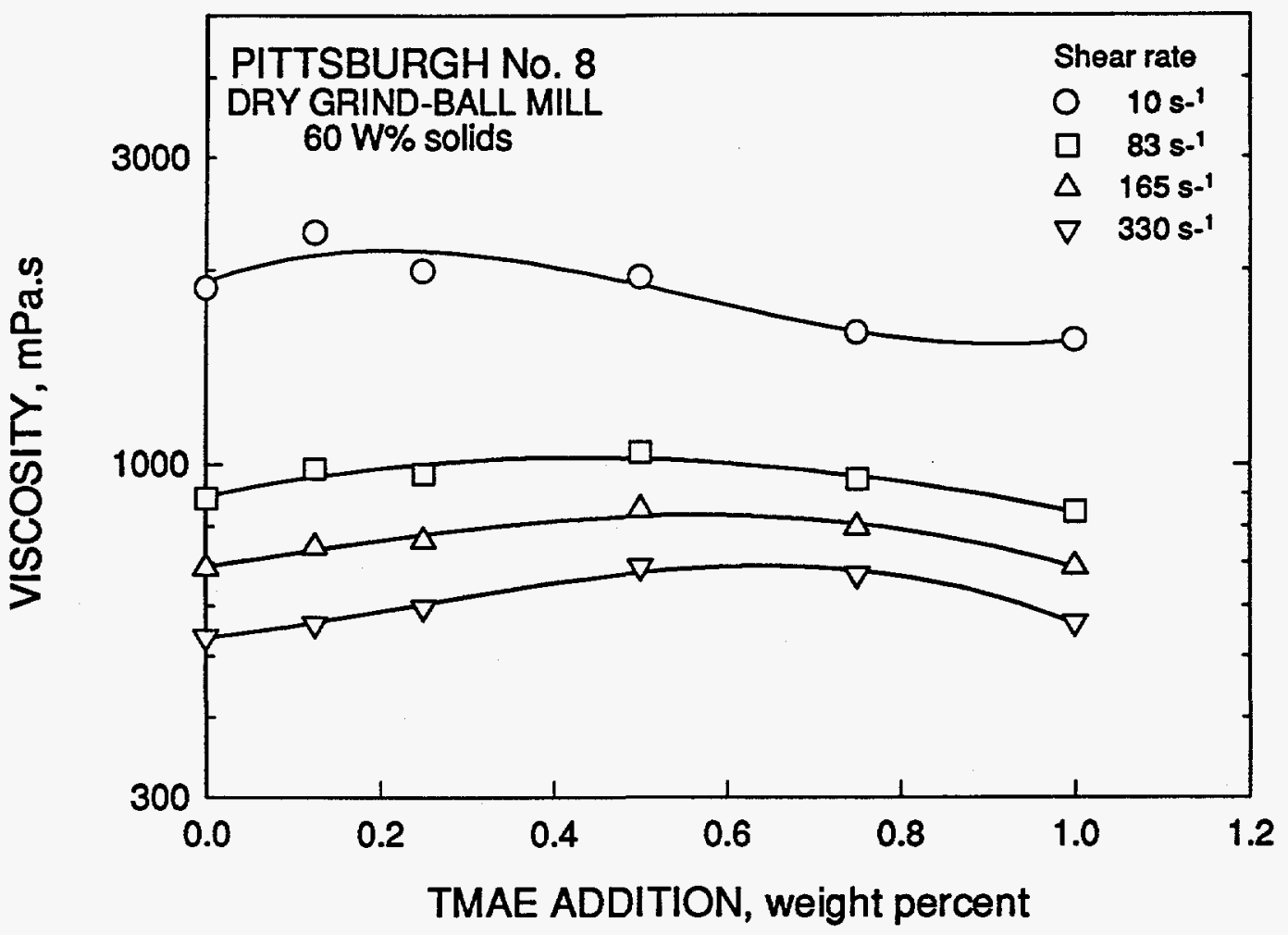

Figure 3.17. Viscosity of Pittsburgh No. 8 coal-water slurries as a function of TMAE addition at four different shear rates.

observed behavior could be the formation and rearrangement of flocs of suitable shape (oblates) that may allow the suspension to flow, thus decreasing the slurry viscosity [30,31].

Figure 3.17 presents the change in viscosity as a function of reagent addition for shear rates of $10 \mathrm{sec}^{-1}, 83 \mathrm{sec}^{-1}, 165 \mathrm{sec}^{-1}$, and $330 \mathrm{sec}^{-1}$. As can be seen from the results given in this figure, the viscosity increases as the reagent addition increases up to $0.5 \mathrm{wt} \%$ and then decreases upon further addition of the reagent. It is important to observe that after an $0.5 \%$ addition of the reagent, the viscosity values never go below those obtained in the absence of this reagent.

So far we have seen that addition of long-chain nonionic surfactants as well as shortchain ionic surfactants results in a significant improvement in the rheology of coal-water slurries. We now present the results of our investigation on the effect of addition of CoalMaster A23-M, a polyelectrolyte, on the rheology of coal-water slurries. Figure 3.18 
shows the effect of CoalMaster addition on slurry rheology. As can be seen from the results given in Figure 3.18a, where the shear stress of slurries are plotted as a function of the shear rate, in the absence of CoalMaster the slurry is pseudoplastic. With the addition of a small dosage ( $0.15 \mathrm{wt} \%$ with respect to solids) of CoalMaster, however, there was a significant drop in the apparent viscosity at shear rate of $100 \mathrm{~s}^{-1}$ from about $2500 \mathrm{mPa}$.s to about 1500 $\mathrm{mPa} . \mathrm{s}$. At $1.2 \mathrm{wt} \%$ CoalMaster addition, the viscosity dropped to $500 \mathrm{mPa}$.s at a shear rate of $100 \mathrm{~s}^{-1}$, with a corresponding change in the flow behavior from pseudoplastic to mildly dilatant which a characteristic of an well-dispersed system.

Figure 3.19 presents a comparative evaluation of the effect of various classes of dispersants on the viscosity of coal-water slurries. The apparent viscosities were normalized with respect to those at zero reagent addition for each reagent system. As can be seen from the figure, CoalMaster is the most effective among the dispersants tested.

\section{EFFECT OF CHEMICAL ADDITIVES ON THE AGGREGATION STABILITY IN COAL-WATER SLURRIES}

To study the effect of chemical additives on the aggregation stability of coal-water slurries and to clarify the explanation suggested for the effect of anionic, cationic and nonionic surfactants on the rheological behavior of Pittsburgh No. 8 coal-water slurries, flocculation tests were performed through indirect measurements that included the relative sedimentation volume and the size distribution of flocs. These measurements were selected because the range of particle size and the high solids content of these slurries do not allow the use of light scattering techniques.

Effect of chemical additives on the particle size distribution in slurries

In order to determine to effect of dispersant addition on the sedimentation volume, slurries were first prepared with various dosages of dispersants. The slurry samples were then transferred to a centrifuge tube and the height of the mud line was recorded after which the 

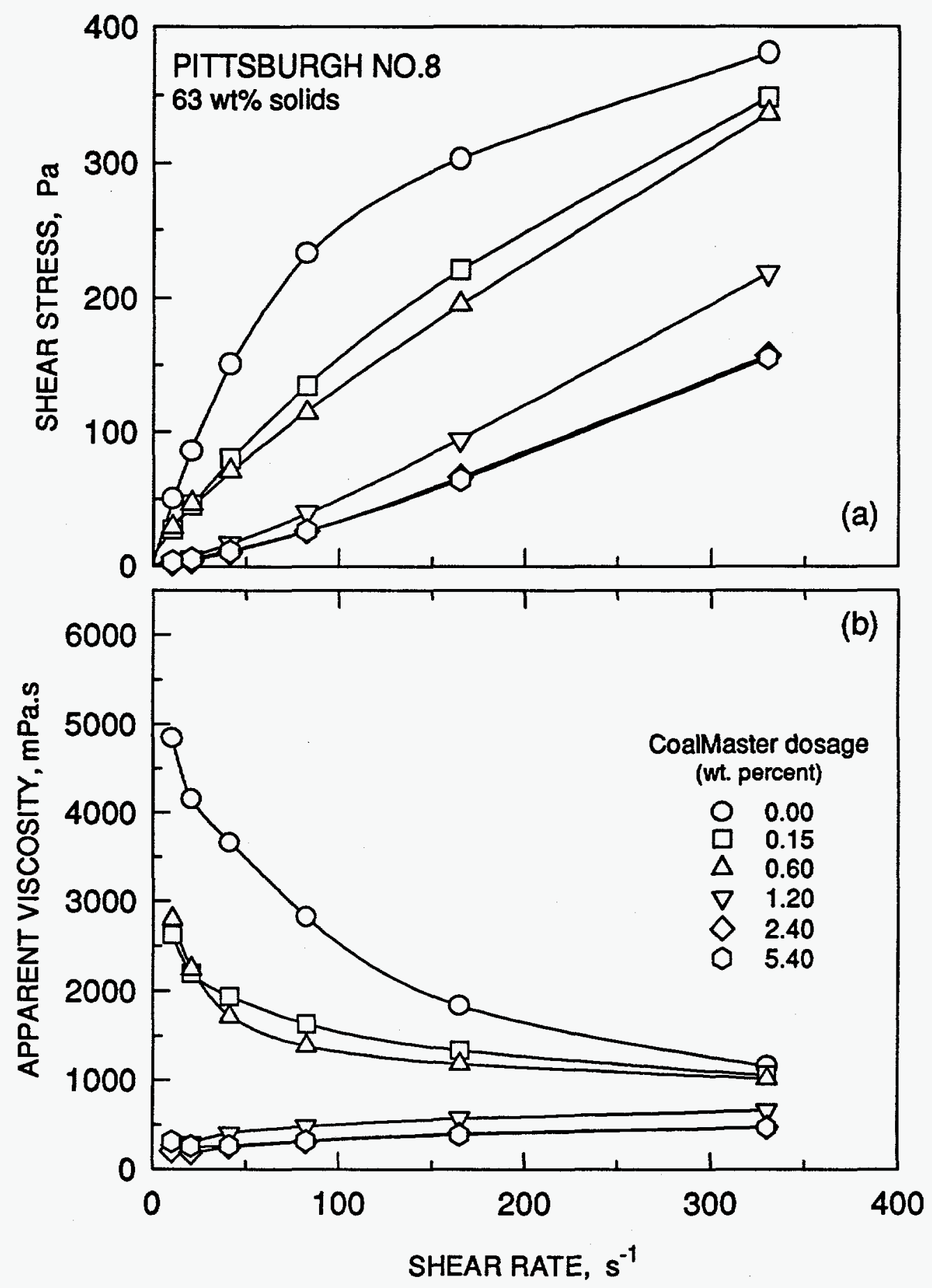

Figure 3.18. (a) Flow curves (theograms) and (b) the viscosity of coal-water slurries prepared with Pittsburgh No. 8 coal and different dosage of CoalMaster A-23M as dispersant as a function of shear rate. 


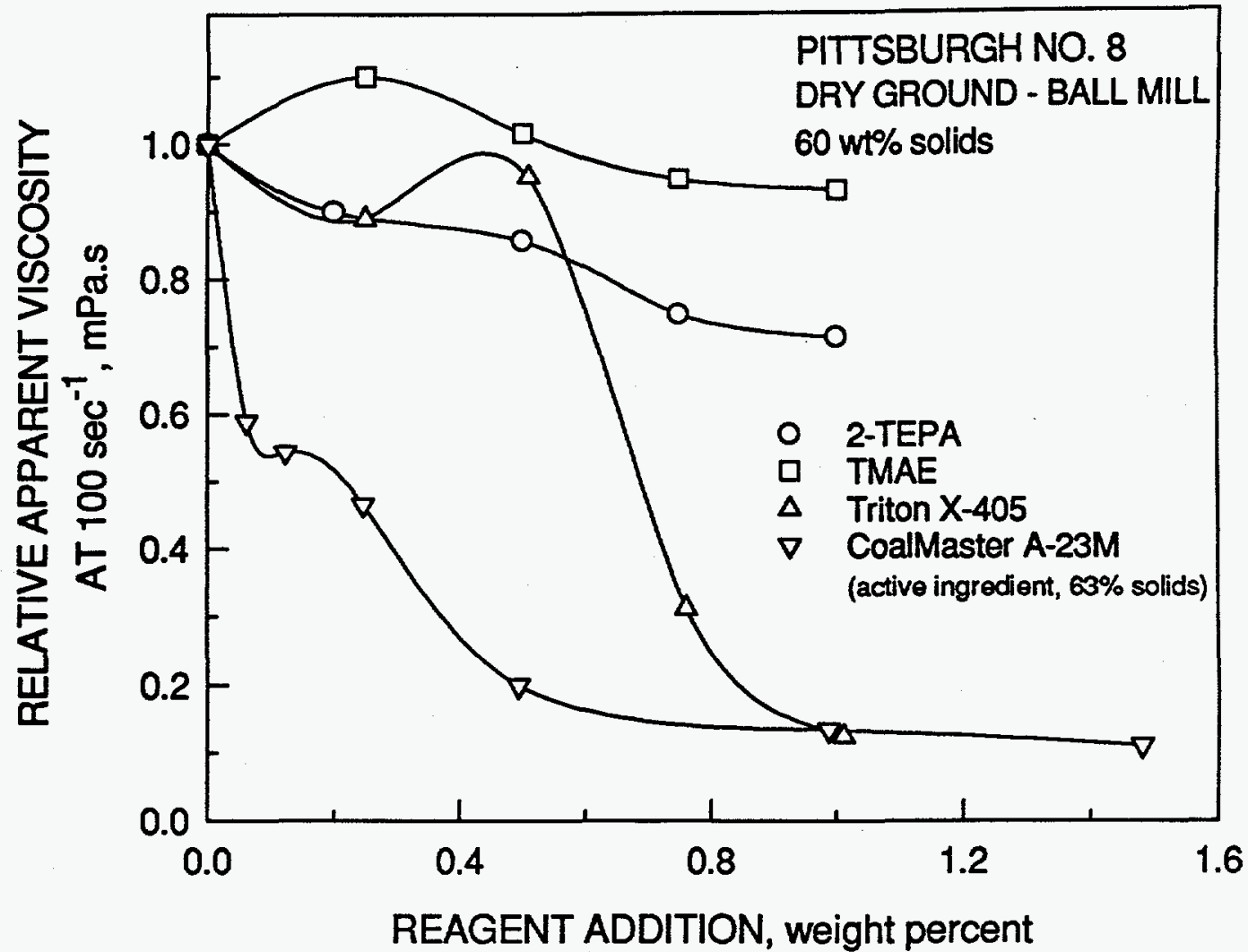

Figure 3.19. A comparative evaluation of the effect of addition of various dispersants on the theology of coal-water slurries.

samples were centrifuged at $16,000 \mathrm{rpm}$ for 30 minutes and the new sediment height recorded. As has been demonstrated by previous researchers [30], the height of the sediment increases as the suspension stability of the slurry decreases, that is, the flocculation of the system is increased.

Figure 3.20 presents the relative sedimentation volume, expressed as a volume percentage of the initial slurry volume, as a function of reagent addition for the anionic 2-TEPA and cationic TMAE surfactants. In the case of 2-TEPA, the relative sedimentation volume decreases as the reagent addition is increased, leveling off after $0.75 \mathrm{wt} \%$ addition of the reagent. These results indicate that the system is dispersed by the presence of this reagent, in agreement with the results of the rheological measurements. In the case of TAME, on the other hand, the relative sedimentation volume decreases slightly at $0.25 \mathrm{wt} \%$ addition, 


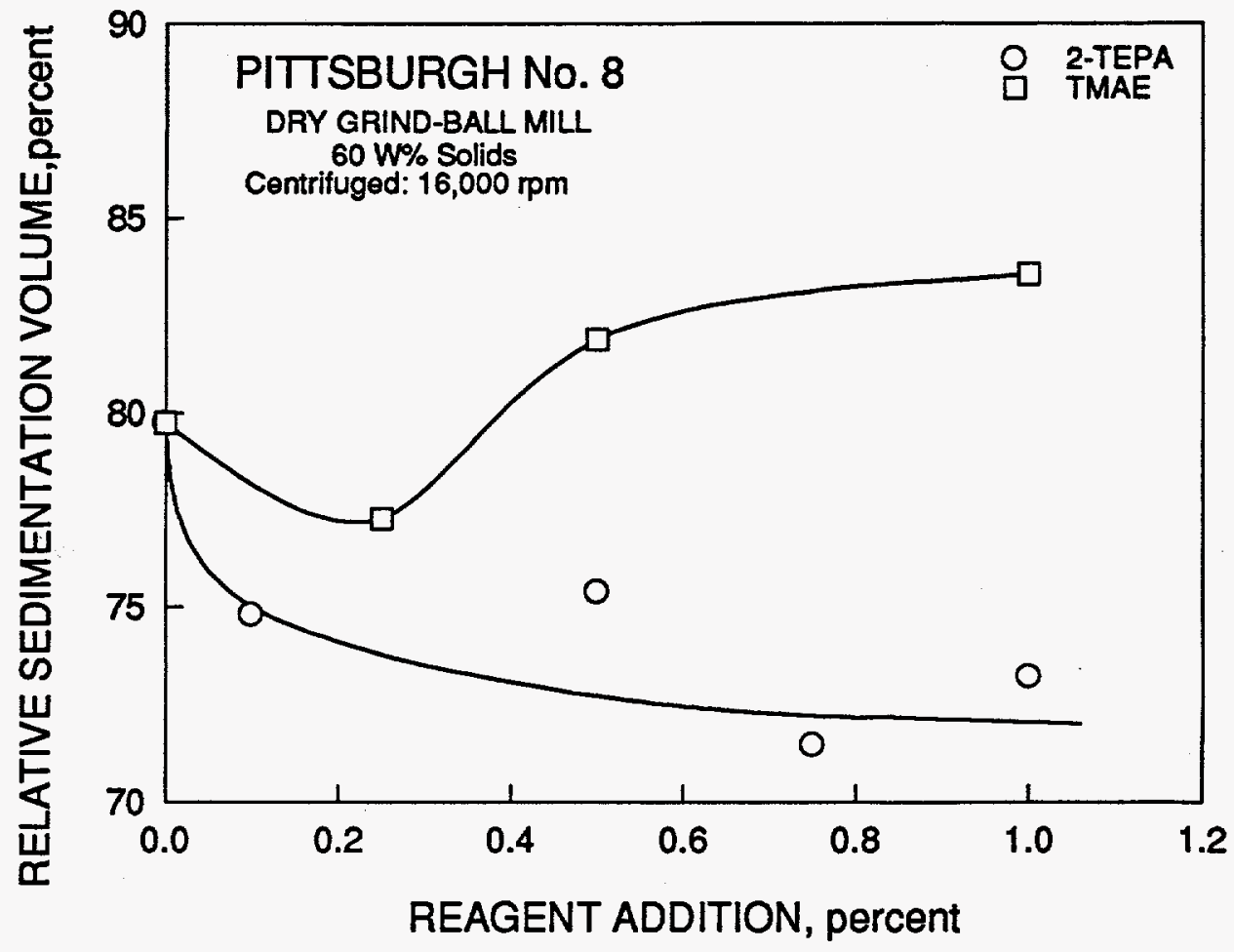

Figure 3.20. Relative sedimentation volume as a function of 2-TEPA and TMAE additions for Pittsburgh No. 8 coal-water slurries ( $60 \mathrm{wt} \%$ solids) centrifuged at $16,000 \mathrm{rpm}$ for 30 minutes.

increasing continuously at higher reagent dosages. Thus, the possible explanations of a weak flocculation and/or a rearrangement of flocculated coal particles may be correct.

Figure 3.21 presents the plots of relative sedimentation volumes of Pittsburgh No. 8 CWS as a function of Triton X-165, X-305 and X-405 dosages. Up to an addition of $0.5 \mathrm{wt} \%$, Triton X-165 does not cause any significant change in the relative sedimentation volume. But higher additions cause a sharp decrease in sedimentation volume. In the case of Triton X-305 and Triton X-405, the relative sedimentation volume steadily decreases as reagents are added to about $0.75 \mathrm{wt} \%$ but then increases again at higher additions.

In the case of nonionic surfactants, the mechanism of interaction of these reagents with the surface of coal is not electrostatic (except for shielding charged sites on the surface by adsorbed neutral molecules). Apparently, the Triton $X$ series of reagents are adsorbed by hydrophobic interaction with the hydrophobic sites on the surface of coal, probably through 


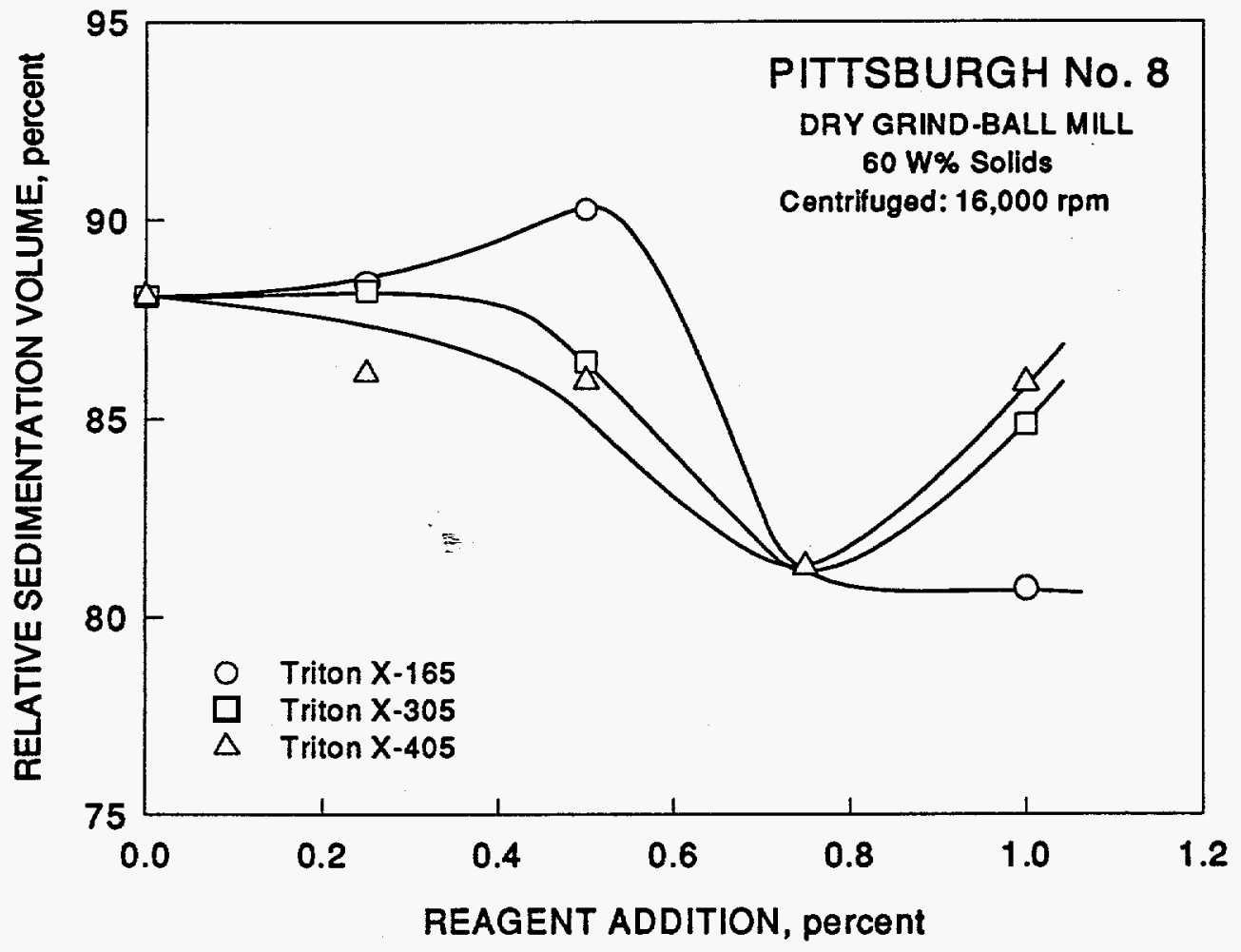

Figure 3.21. Relative sedimentation volume as a function of Triton $X$ series reagents addition for Pittsburgh No. 8 coal-water slurries ( $60 \mathrm{wt} \%$ solids) centrifuged at $16,000 \mathrm{rpm}$ for 30 minutes.

their aromatic groups. Consequently, the hydrophilic ethylene oxide groups of these nonionic surfactants orient towards the bulk liquid, tending to increase the water layer on the coal particle $[29,32]$. At low reagent dosage, which corresponds to low surface coverage, these reagents are not able to increase the hydration layer significantly, maintaining the system in a weakly flocculated state. However, at higher reagent additions, it appears that the aggregation stability of these systems increases, possibly due to a thick hydration layer over the coal particles. The increase in the sedimentation volume for slurries prepared with $1 \mathrm{wt} \%$ addition of Triton X-305 and Triton X-405, which contain 30 and 40 ethylene oxide groups, respectively, may be due to the formation of a bilayer (reverse micelles) which tends to make the system weakly flocculated (increase in the relative sedimentation volume). In the case of Triton X-165, which contains only 16 ethylene oxide groups, no increase of the relative 
sedimentation volume was observed, even at $1 \mathrm{wt} \%$ addition probably because the amount of reagent added was not enough to form a bilayer (reverse micelles) over the surface.

\section{Effect of chemical additives on the particle size distribution in slurries}

Measurement of the sedimentation volume of slurries has provided us with an indirect estimate of the state of aggregation of particles in the slurries. We will now present the results of direct measurement of the particle size distribution of particles in the slurries as a function of reagent addition. The aggregates, in general, are weak and to avoid the dispersion of the system experiments with an Andreasen pipette, which is a simple gentle sedimentation technique, were carried out for determining particle size distributions. An assumed density of flocs equal to the density of coal was used in determining floc size distributions. Although the size distributions thus calculated are not the true size distributions, they provide a method for comparing flocculation response in the different experiments. Figures 3.22 to 3.24 present the apparent particle size distribution for ground Pittsburgh No. 8 in the absence and presence of 2-TEPA, TMAE and Triton X-305, respectively. As can be seen from these figures, the size distributions are unimodal and fairly symmetric about the mean sedimentation diameter for each of the distributions, with a slight increase in the spread of the distribution with the increase in the reagent concentration. Therefore, monitoring the change in the mean sedimentation diameter as a function of the reagent dosage should result in a fair assessment of the effect of chemical additives on the particle size distributions of slurries.

As can be seen from Figure 3.22, in the case of 2-TEPA addition observed Stokes' diameter or sedimentation diameter decreases with increasing reagent addition, indicating that this reagent disperses the system. These results are in agreement with the rheological data which show that the viscosity decreases as the reagent addition increases. On the other hand, the results given in Figure 3.23 for the case of TMAE indicate no change in the sedimentation diameter (except for apparent aggregation in the absence of reagent). Since sedimentation 


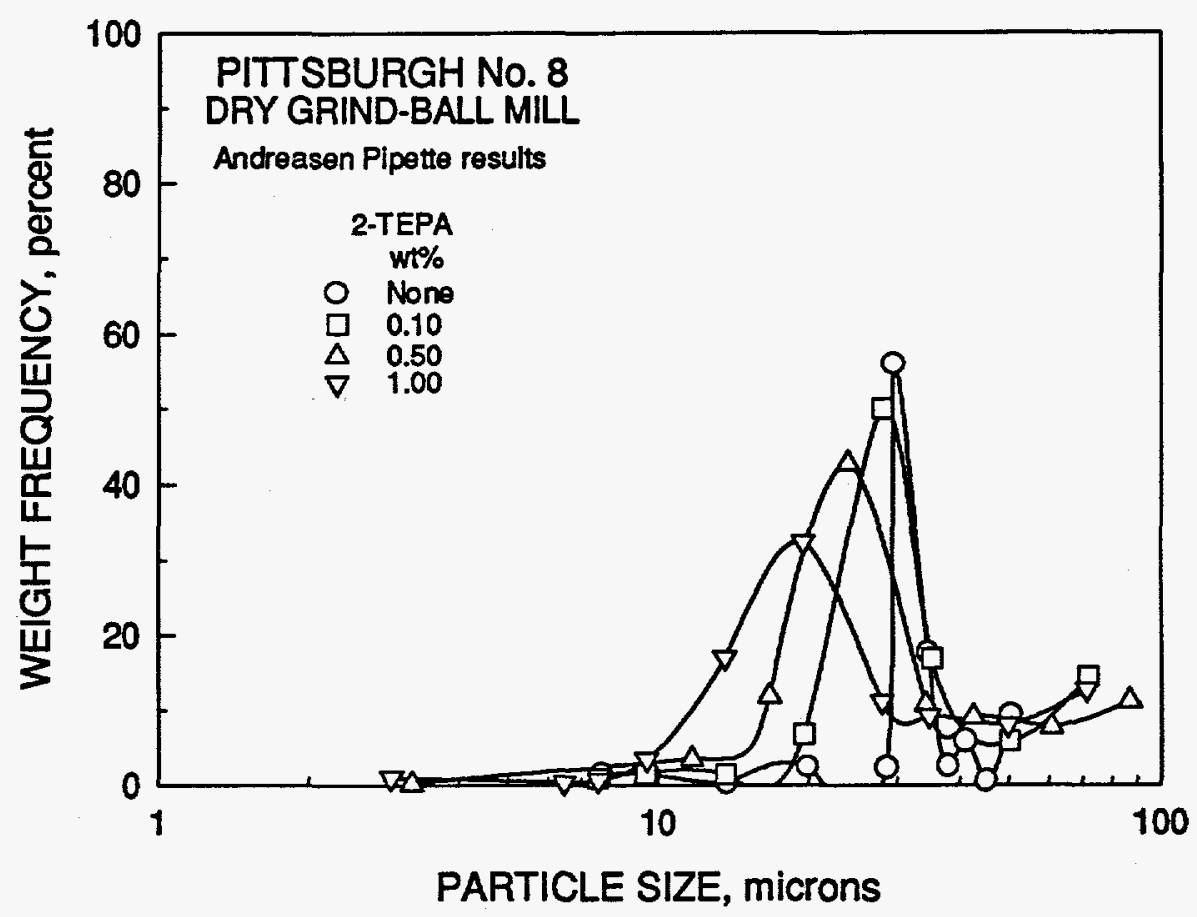

Figure 3.22. The effect of 2-TEPA addition on the particle size distribution, determined by the Andreasen pipette technique, of slurries prepared with Pittsburgh No. 8 coal.

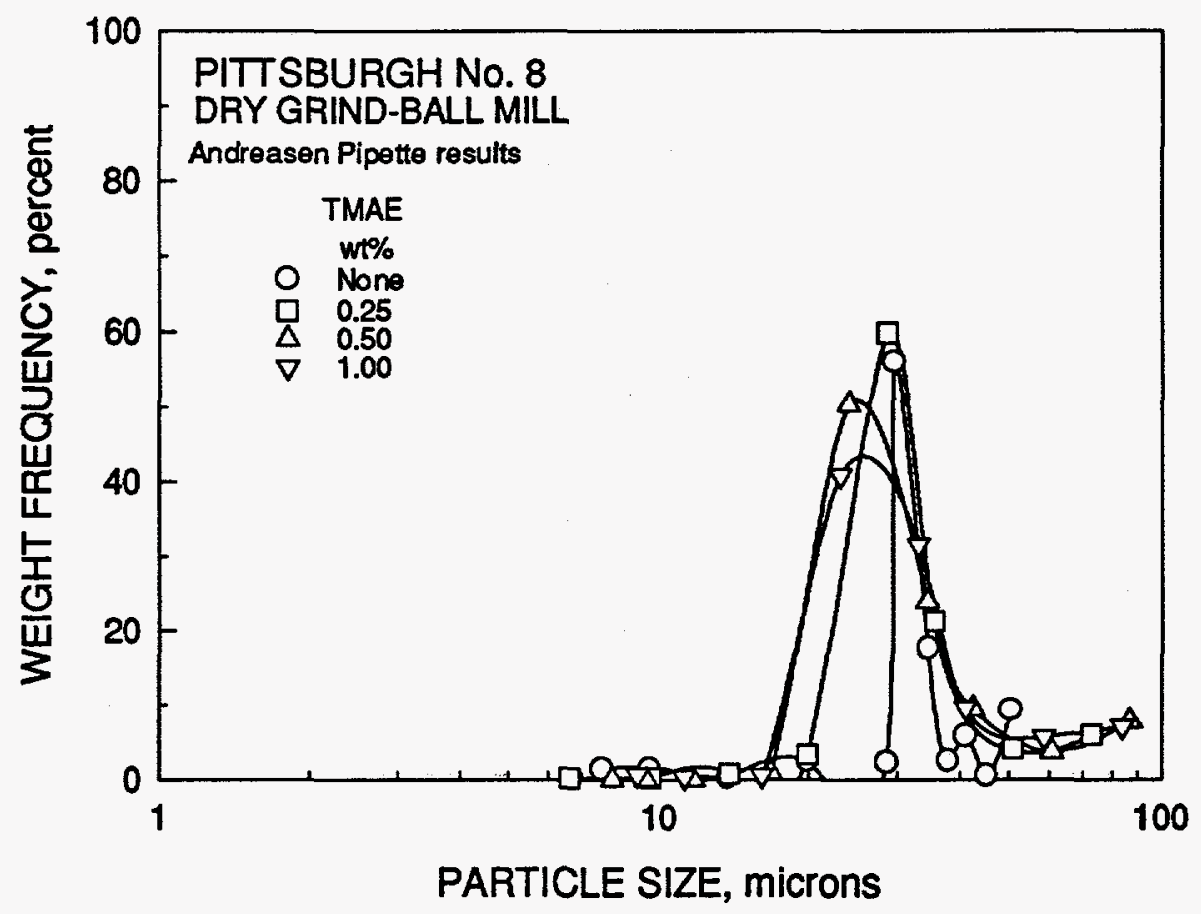

Figure 3.23. The effect of TMAE addition on the particle size distribution, determined by the Andreasen pipette technique, of slurries prepared with Pittsburgh No. 8 coal. 


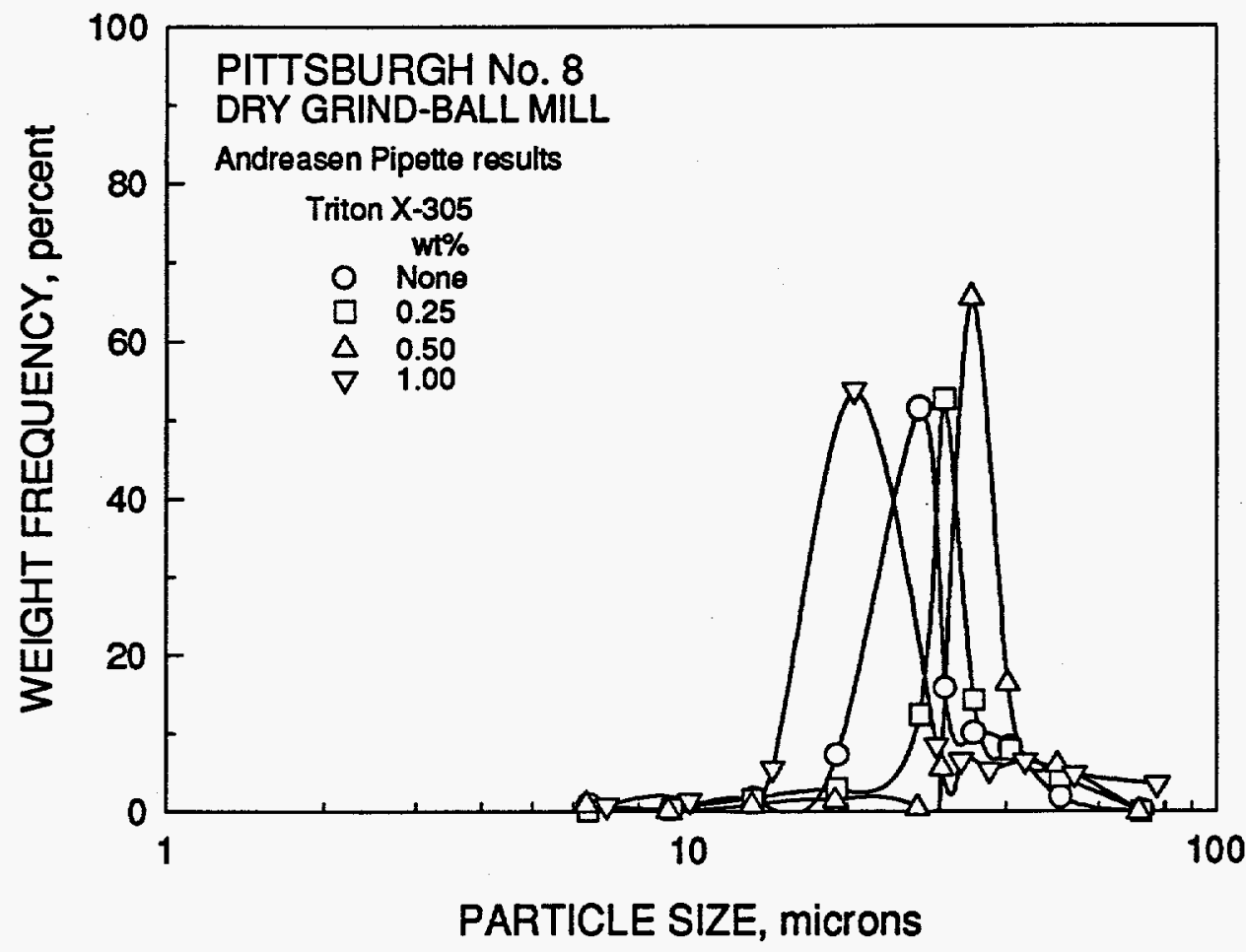

Figure 3.24. The effect of Triton X-305 addition on the particle size distribution, determined by the Andreasen pipette technique, of slurries prepared with Pittsburgh No. 8 coal.

techniques are based on determining the settling rate of particles according to Stokes' law (which is based on the equilibrium between gravity and drag forces), the presence of weak flocs could reduce the density of the aggregate, resulting in a settling rate for the aggregate equivalent to that for the particles in the absence of the reagent. Another possible explanation for the behavior of the system in the presence of TMAE could be related to the shape of the flocs formed. The settling rate of the aggregates can decrease if they form an oblate floc (nonspherical), which increases the drag force when settling. Apparently, the presence of oblate aggregates could explain the observed decrease in viscosity at high TMAE addition due to the alignment of the flocs in the direction of the flow [31]. Therefore, the apparent change in floc diameter due to reagent that promotes a weak flocculation of the system, such as in the case with TMAE, could not be detected by the Andreasen pipette technique. This can be seen by the results given in Figure 3.25, which presents the sedimentation mean diameter as a 


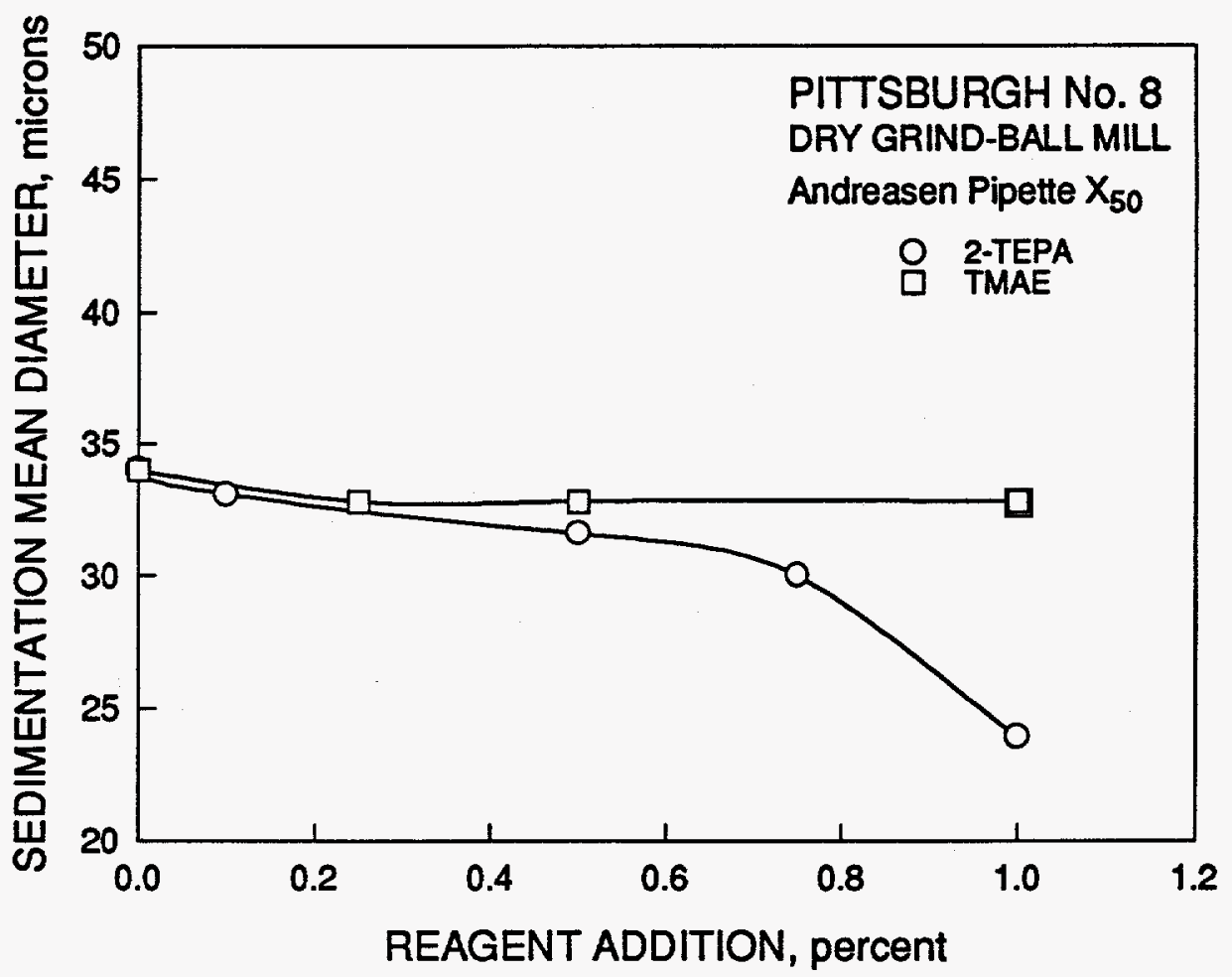

Figure 3.25. Sedimentation mean diameter as a function of reagent addition for Pittsburgh No. 8 coalwater slurries in the absence and presence of 2-TEPA and TMAE.

function of 2-TEPA and TMAE addition for Pittsburgh No. 8 coal-water slurries. These plots show that 2-TEPA disperses the system, whereas the cationic TMAE does not show any apparent change in the mean sedimentation diameter.

The effects of the various Tritons on the size distribution of flocs are similar. Figure 3.24 shows the apparent particle size distribution in the absence and presence of Triton X305. As can seen from the plots in the figure, the observed Stokes diameters or sedimentation diameters increase with the addition of nonionic surfactants up to $0.5 \mathrm{wt} \%$, indicating flocculation of the system. However, at higher nonionic surfactant addition, the sedimentation diameter decreases, indicating dispersion of the system. These results are in agreement with those obtained by the relative sedimentation volume technique. However, the results of the particle size distribution measurements do not indicate the degree of flocculation of the system that is shown by the sedimentation volume tests at $1 \mathrm{wt} \%$ addition. This is probably beacuse 


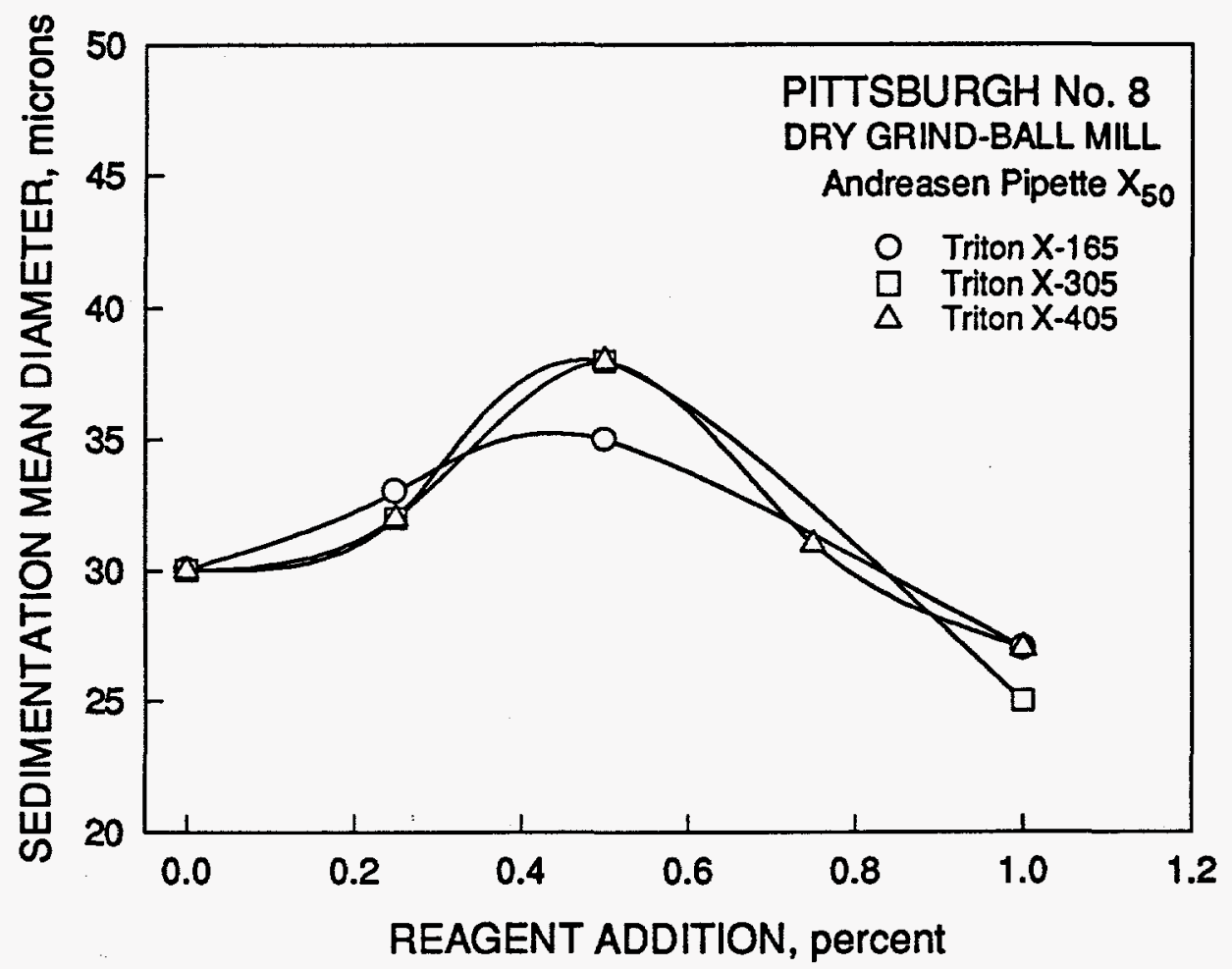

Figure 3.26. Sedimentation mean diameter as a function of reagent addition for Pittsburgh No. 8 coal-water slurries in the absence and presence of Triton X-165, X-305 and X-405.

of the inability to detect the apparent change in floc diameter due to the weak flocculation of the system by the Andreasen pipette technique.

The sedimentation mean diameters for Pittsburgh No. 8 coal-water slurries in the presence of Triton X-165, Triton X-305 and Triton X-405 are presented in Figure 3.26. This figure shows that the sedimentation mean diameter increases with the addition of Tritons from $30 \mu \mathrm{m}$ in the absence of reagents to about $35-38 \mu \mathrm{m}$ at $0.5 \mathrm{wt} \%$ (flocculation) and then decreases to about $25-27 \mu \mathrm{m}$ with the addition of $1 \mathrm{wt} \%$ (dispersion). Apparently, the addition of Triton reagents over $0.5 \mathrm{wt} \%$ results in the dispersion of the system due to the shielding of the particles by these nonionic surfactants, reducing particle-particle interactions.

Our studies on the agggreagation stability of coal-water slurries in the presence of chemical additives have shown that, in general, there is a fairly good correlation between the aggregative stability, as determined by either sedimentation volume or particle size measurement, with a few exceptions. For instance, in case Tritons, increasing the reagent 


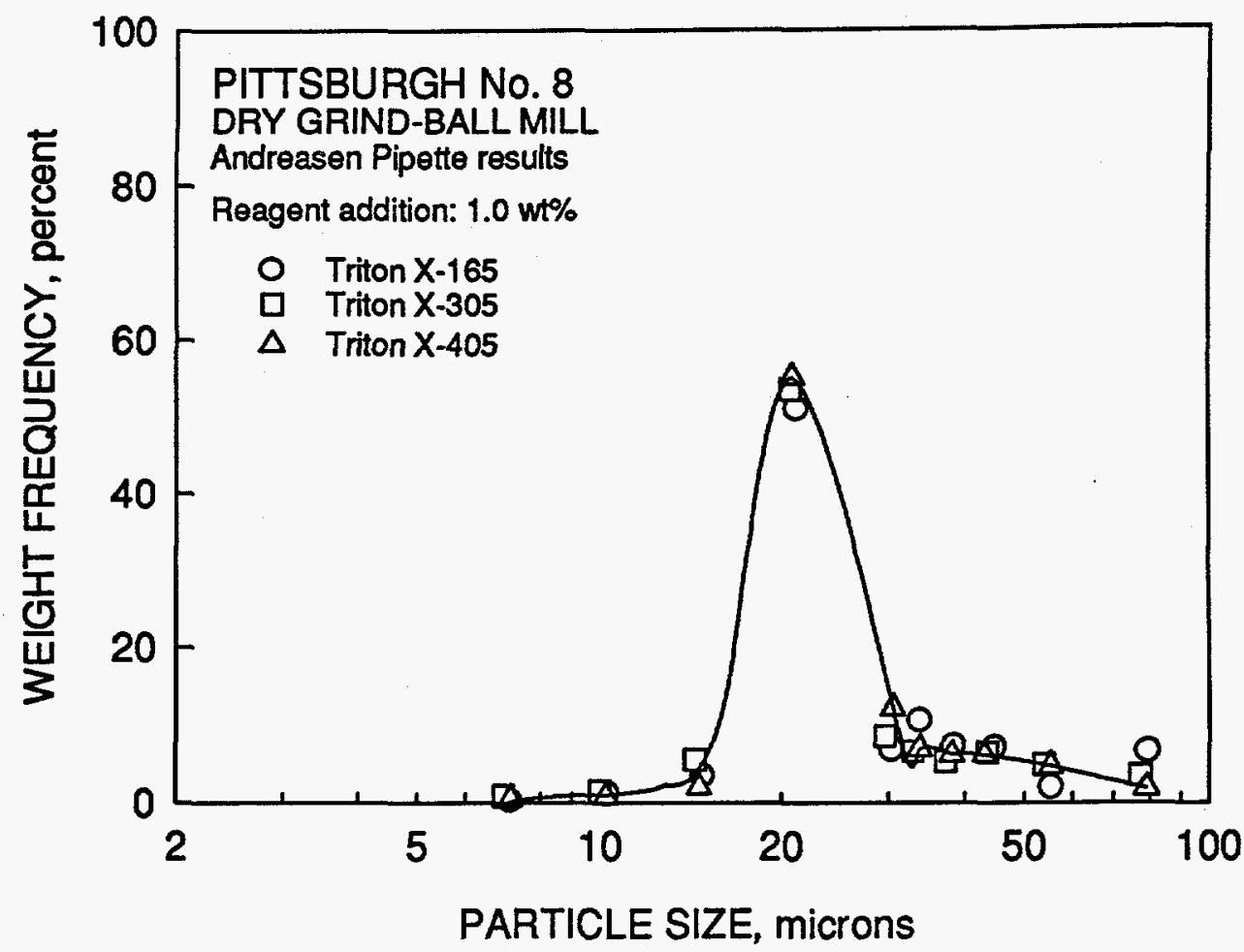

Figure 3.27. Apparent particle size distribution for Pittsburgh No. 8 coal-water slurries in the presence of $1 \mathrm{wt} \%$ reagent.

dosage from 0.75 to $1 \mathrm{wt} \%$ results in an increase in the sedimentation volume, indicating a decrease in the aggregative stability of slurries, except for Triton X-165 which shows no such increase. Rheological measurements, however, show a continuous decrease in the viscosity of slurries with an increase in the Triton dosage from $0.5 \mathrm{wt} \%$ to $1 \mathrm{wt} \%$, which implies an increase in the aggregative stability of the slurries over this range of reagent addition. The sedimentation mean diameter, on the other hand, follows the same trend as the viscosity as a function of the Triton dosage. Moreover, as shown in Figure 3.27, which presents the apparent size distributions obtained using the Andreasen pipette technique for $1 \mathrm{wt} \%$ addition of the three Tritons, the three Tritons result in nearly identical particle size distributions at $1 \mathrm{wt} \%$ reagent dosage. The viscosities of slurries at this dosage of the different Tritons are also about the same, indicating that $1 \mathrm{wt} \%$ reagent addition the three Tritons result in similar aggregative stability of coal-water slurries. 


\section{WETTING BEHAVIOR OF COAL AND RHEOLOGY OF COAL-WATER SLURRIES}

The formation of water film around coal particles plays an important role in determining the rheological behavior of the slurries. Insufficient film formation can result in increased interparticle friction as well as increased probability of aggregate formation, both of which will result in higher viscosity of slurries. The effect of CoalMaster on the wetting behavior of coal and the rheology of slurries was therefore investigated.

In order to ascertain the effect of Coalmaster on the wetting behavior of coal, contact angle measurements were carried out with the captive bubble method on a polished coal sample. A polished coal sample was immersed in an aqueous solution at a given CoalMaster concentration and an air bubble was made to contact the solid surface. The contact angle was measured directly using a comparator microscope with a goniometer scale. The procedure was repeated for various CoalMaster concentrations. The measured contact angles as a function of CoalMaster contentration are plotted in Figure 3.28. The contact angle drops

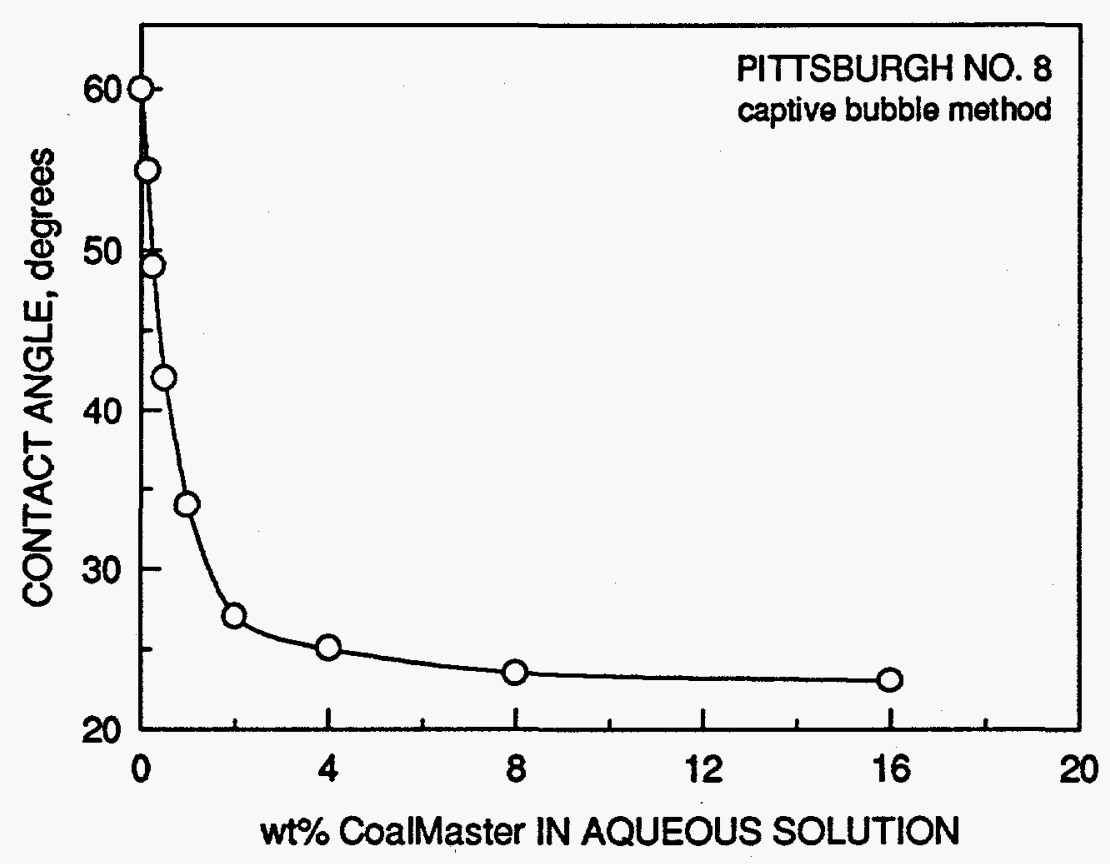

Figure 3.28. Effect of CoalMaster concentration on the contact anlge of Pittsburh No. 8 coal, measured by captive bubble method. 
sharply at small concentrations of CoalMaster and eventually levels off at about 23 degrees indicating that CoalMaster strongly wets the coal surface.

In order to establish the relationship between the wetting of coal particles by CoalMaster and the rheology of coal-water slurries, slurries were prepared with different dosage of CoalMaster. Since CoalMaster adsorbs on coal, which will change the equilibrium concentration of CoalMaster in the aqueous phase of the slurry and hence the degree of wetting of coal particles, the concentration of CoalMaster in the aqueous phase of these slurries was measured along with the theological measurements. A plot of the viscosity of these slurries at shear rate of $100 \mathrm{~s}^{-1}$ as a function of equilibrium CoalMaster concentration is superimposed on the contact angle plot in Figure 3.29. It is evident from the figure that there is a direct correspondence between the wetting of coal and the rheology of coal-water slurries.

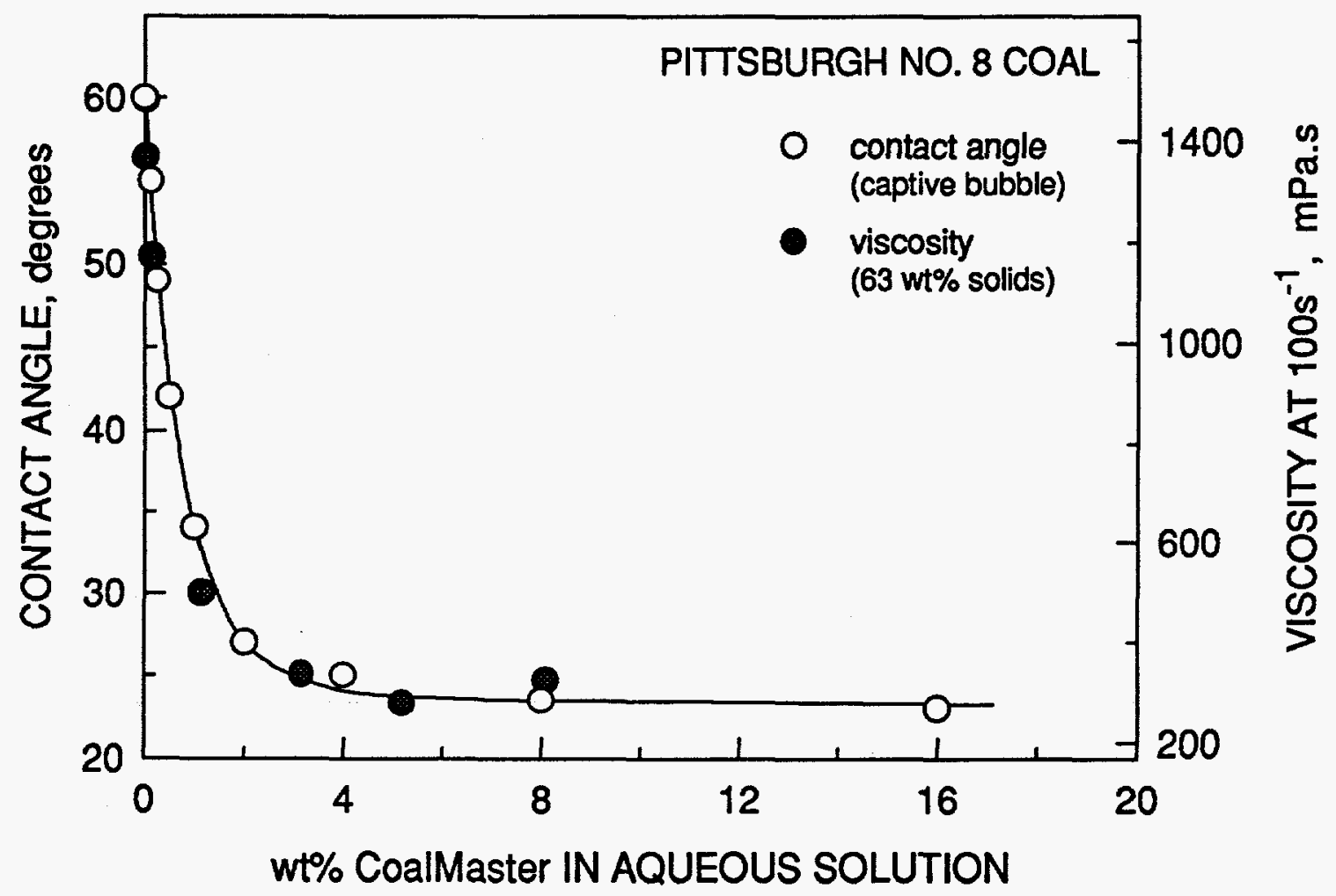

Figure 3.29. The correlation bewteen the wetting characteristic of coal and the rheological behavior of coal-water slurries. 


\section{SUMMARY}

The results of our studies on the rheology of coal-water slurries prepared with ball mill ground coal have been presented in this section. These studies were carried out in order to establish the baseline against which the rheological behavior of coal-water slurries prepared with high-pressure roll milled coal could be compared in order to delineate the effect highpressure roll milling of coal on the rheological behavior of slurries.

Electrokinetic studies were performed to determine the effect of surface charge characteristics of coal particles on the rheology of slurries. The effect of addition of ionic as well as nonionic dispersants on the rheology of slurries was investigated in detail. Our studies showed that among the various reagents tested, CoalMaster A-23M, an anionic polyelectrolyte, results in maximum improvement in slurry rheology. Wettability studies showed that the superior performance of CoalMaster results from its ability to strongly wet the coal surface. CoalMaster was therefore chosen as the primary dispersant of further studies.

The effect of the addition of anionic, cationic and nonionic surfactants on the aggregation stability of coal-water slurries was investigated by determination of sedimentation volume and the floc size distribution. Our studies also showed that there is a fairly good correlation between the aggregative stability of slurries, as determined by sedimentation volume measurements as well as by apparent particle size measurements, and their rheological behavior. 


\section{SECTION IV}

\section{HIGH-PRESSURE ROLL MILL GRINDING AND RHEOLOGY OF SLURRIES}

In order to prepare coal-water slurries having sufficient stability, suitable theological behavior and good combustibility, the raw coal must be ground to a fine size (say, a median size of $40 \mu \mathrm{m}$ or less) and the product should have a size distribution corresponding to dense packing density. Because fine grinding is an energy-intensive process, grinding costs represent a substantial portion of the total cost in advanced coal beneficiation and in coalwater slurry preparation. High grinding energy consumption and difficulty in obtaining a ground product size distributions corresponding to maximum packing density constitute significant problems in the manufacture of coal-water slurries with conventional mills, such as the tumbling ball mill and stirred ball mill.

Perhaps, the most promising innovation in fine grinding in recent years has been the development of the choke-fed, high-pressure roll mill in 1985 which within a few years has been proven to be the most efficient grinding mill in the field of cement clinker grinding. Confined particle-bed comminution, as realized in high-pressure roll milling, also produces ground products that have desirable packing characteristics. In particle-bed comminution, when the pressure in the bed increases, edges and corners of larger particles break off preferentially. Smaller particles produced this way fill the voids between the larger ones. If the pressure applied is high enough, this process stops only after most voids are filled up by smaller particles. As a consequence, interparticle crushing automatically produces a particle size distribution corresponding to the near maximum packing density. This phenomenon is of great importance in the preparation of coal-water slurries whose rheology is known to depend strongly on the packing characteristics of the solid particles.

The principal objective of this research work was to systematically investigate the energy consumption and the stability and rheology of coal-water slurry fuel prepared with a laboratory-scale, choke-fed, high-pressure roll mill, and to compare the results with slurry 
prepared with coal that had been comminuted with a conventional grinding mill (such as the ball mill).

\section{HIGH-PRESSURE ROLL MILL GRINDING OF COAL}

In order to study the energetics of the high-pressure roll milling of coal, minus $1 / 4$ inch coal sample was ground in the high-pressure roll mill at various levels of energy input. Because of the plastic nature of coal and the intense localized stresses encountered by the particles during the comminution process, except at low levels of energy inputs, high-pressure roll mill products were usually in briquetted form. Therefore, to ascertain the true extent of grinding that has taken place in the roll gap prior to the briquetting of the ground mass, a deagglomeration procedure had to be developed, a technique that would not further comminute the coal. Preliminary tests showed that the agglomerates were generally strong enough to resist complete disintegration during wet-sieving or by ultrasonic treatment in a water slurry. It was then decided to use methanol (which had been earlier identified as a good dispersing agent for coal) because of its wetting properties. However, it soon became apparent that breaking up the agglomerates completely by just soaking them in methanol was also not possible. To overcome this problem, the agglomerates were stirred in a methanol suspension (100 grams of coal plus $100 \mathrm{ml}$ of methanol) at 900 RPM for different time intervals in a beaker. After the stirring procedure, the size distribution of the samples were obtained by first wet sieving at 200 mesh, then drying the material, and dry sieving the plus 200 mesh material for 20 minutes on a series of Tyler sieves, using a Ro-Tap sieve shaker.

Figure 4.1 shows the size distributions of Pittsburgh No. 8 coal, plotted on log-log scale as the cumulative percent finer than the stated size (Gaudin-Schuhmann plots), that had been ground in the high-pressure roll mill with different energy inputs. The roll mill products were deagglomerated by stirring in methanol for 30 minutes. As expected, the size distribution curves shift toward finer size with increased energy investment in the highpressure roll mill. The size distributions are self-similar, as can be seen from Figure 4.2, 


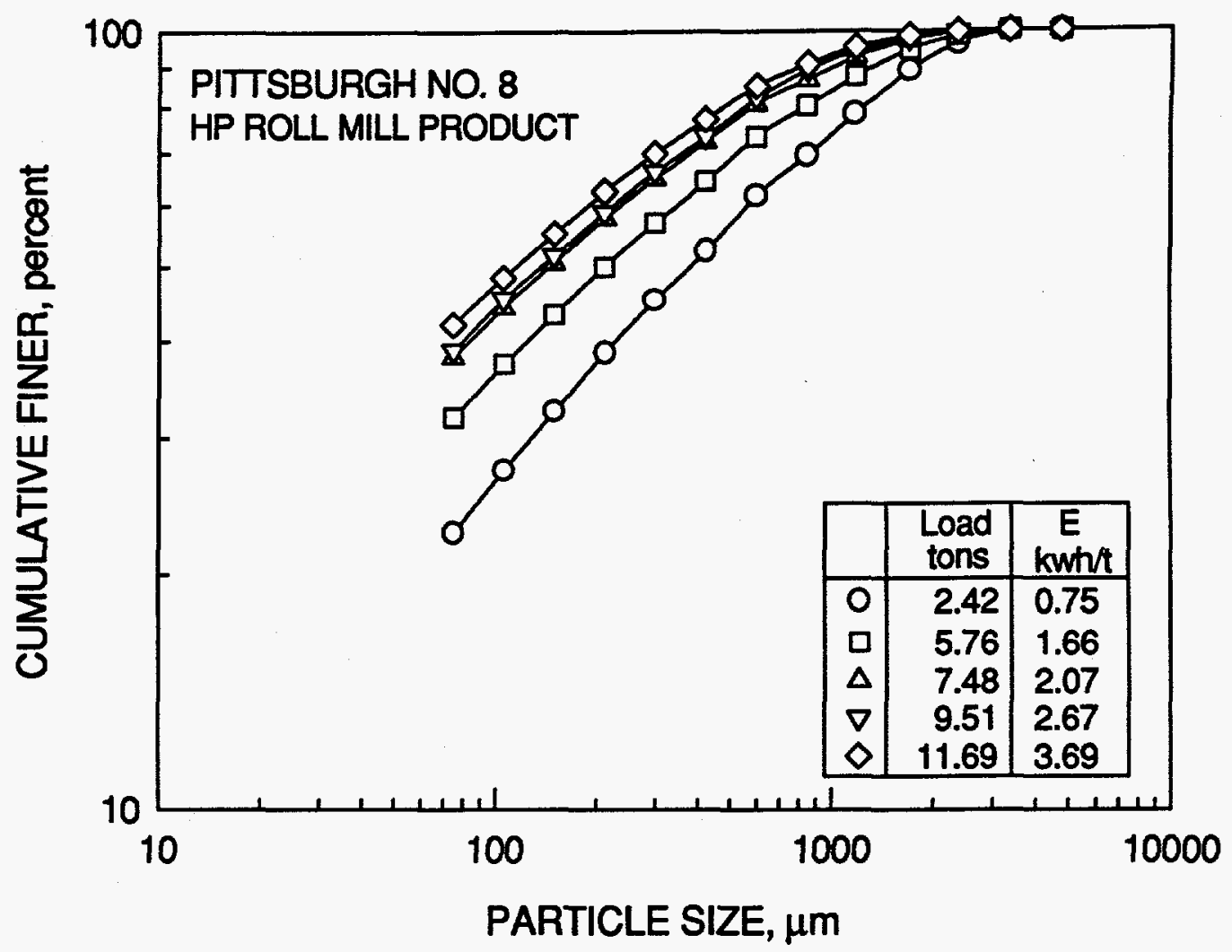

Figure 4.1. Size distribution of Pittsburgh No. 8 coal ground in the high-pressure roll mill at different energy expenditures.

where the distributions are replotted as a function of a dimensionless size, that is, particle size rescaled by the median size. The size distributions of three ground products invariably tend to be self-similar or self-preserving, whether the material is ground in a ball mill (or media mill) [33], or crushed in a single-particle mode [34], or comminuted in a high-pressure roll mill [35]. This characteristic of the size distributions is in fact a consequence of the geometrically similar crack patterns formed whose scale become progressively finer with increasing strain energy in the solid medium. The self-similar size distribution can always be expressed as [33]:

$$
F(x ; E)=\bar{Z}\left(\frac{x}{X_{50}}\right)
$$

where $F$ is the distribution and density function after an expenditure of specific energy $E$ and $\bar{Z}$ and is the self-similar distribution function in the rescaled dimensionless size $x / X_{50}$. An 


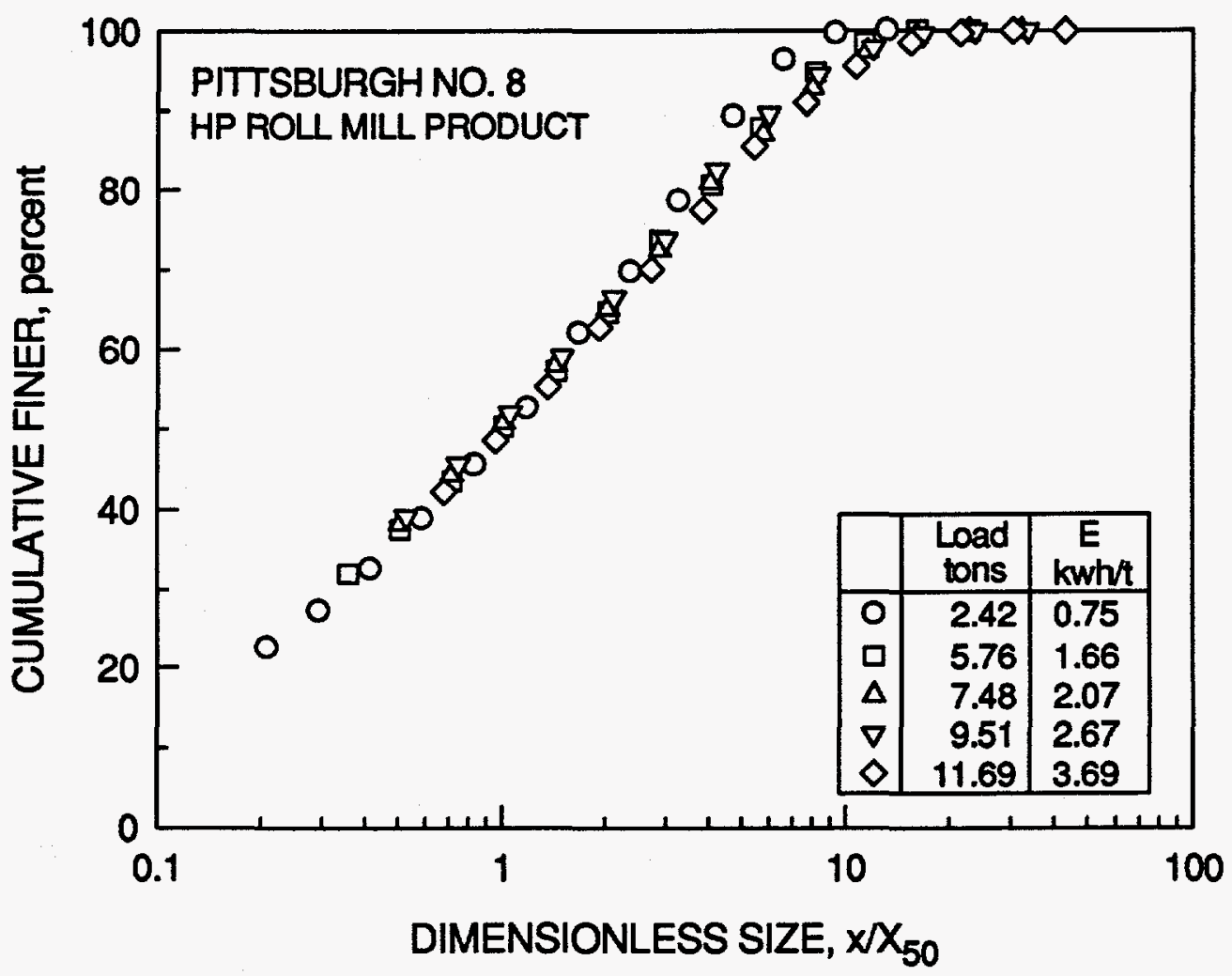

Figure 4.2. Self-similarity of product size distributions of Pittsburgh No. 8 coal ground in the high-pressure roll mill at different energy expenditures.

important implication of the self-similar nature of the size distributions is that the size spectrum is driven forward on its trajectory solely by reduction in one parameter, namely, the median size $X_{50}$. Consequently, the median size is a valid and consistent one-parameter measure of the product fineness.

While high-pressure roll mill grinding results in a wider product size distribution, implying better packing characteristics, and compared to that produced by ball milling, even at $3.7 \mathrm{kWh} / \mathrm{t}$ of energy input, however, the roll mill product contained only 40 percent of minus 200-mesh particles, which is much lower than 80 percent of minus 200-mesh particles required in the coal-water slurries for direct combustion in power plants. Therefore, dagglomeration and/or further grinding of the high-pressure roll milled product in ball mill or rod mill is essential. 
Because high-pressure roll mill comminution of coal results in the generation of highly fractured, stressed or otherwise weakened daughter particles, the deagglomeration of the briquettes and further reduction in particle size can be achieved by grinding the roll mill product in a ball mill with moderate additional energy expenditure. Grinding coal first in the high-pressure roll mill and then in a ball mill, a configuration we refer to as the hybrid circuit configuration, could lead to significant savings in the overall energy expenditure for comminution, as compared to that required for grinding coal to any given degree of fineness in a ball mill alone.

\section{HYBRID GRINDING OF COAL}

Particle-bed comminution of primary coal particles under compressive stresses in the high-pressure roll mill results in highly fractured and weakened daughter particles. The degree of fracturing and weakening of the particles increases with the applied load during highpressure roll mill grinding. However, due to the visco-plastic nature of bituminous coal, the product from the high-pressure roll mill comes out briquetted and the degree of briquetting increases with the applied load. The two opposing influences of the increase in the applied load on the strength characteristics of high-pressure roll mill products suggest that there should an optimal load, and hence energy input, in the high-pressure roll mill for maximum energy saving. In order to determine the optimal energy input, we need to have a proper understanding of the influence of energy input in the high-pressure roll mill on the kinetics of grinding of the roll mill product in the ball mill. The ultimate objective is to estimate the expected saving in the energy expenditure in hybrid closed-circuit continuous grinding operations relative to that in closed-circuit air-swept ball mill and roll / race mill grinding of coal, the traditional industrial practice for fine grinding of coal.

To achieve these goals, the research work was divided into two sections :

1. Investigation of the open-circuit grinding kinetics of the high-pressure roll mill product in the ball mill, and 
2. Simulation of continuous steady state operation of closed-loop hybrid grinding systems through batch locked-cycle grinding experiments as well as predictive computer simulation based on batch grinding kinetics data.

These studies should allow us to identify the best hybrid circuit configuration and the energy split between the high-pressure roll mill and the ball mill for optimal performance of the coal grinding circuit.

\section{Kinetics of Open Circuit Hybrid Grinding of Coal}

Preliminary investigations on the high-pressure roll mill grinding of coals have indicated that for comparable energy inputs, high-pressure roll milling of coal results in a significantly higher production of fines compared to that obtained by ball mill grinding. However, the high-pressure roll mill product, which comes out briquetted, had to be first deagglomerated in order to obtain the product size distribution. The briquettes were deagglomerated by stirring in methanol during the preliminary studies. In industrial practice though, deagglomeration would have to be achieved by mechanical means, using either hammer or media mills. Therefore, in order to obtain a quantitative measure of the overall energy consumption and the kinetics of fine grinding of coal using high-pressure roll mills, a series of batch grinding tests was carried out employing a high-pressure roll mill - ball mill combination, referred to as the hybrid grinding system in this report.

In this series of grinding experments, the high-pressure roll mill product was ground in our test ball mill which has been specially fabricated and instrumented for accurate measurement of energy consumption under standardized grinding conditions. In the tests, minus 1/4-inch coal feeds were first ground in the high-pressure roll mill at various input energy levels. The roll mill product was then ground in the ball mill for increasing lengths of time, typically starting at half a minute and successively doubling of the time period for each subsequent tests. The ball mill discharge was dry-sieved in a Ro-Tap machine for 20 minutes to obtain the size distribution. In addition, the minus $1 / 4$-inch feed was ground directly in the 


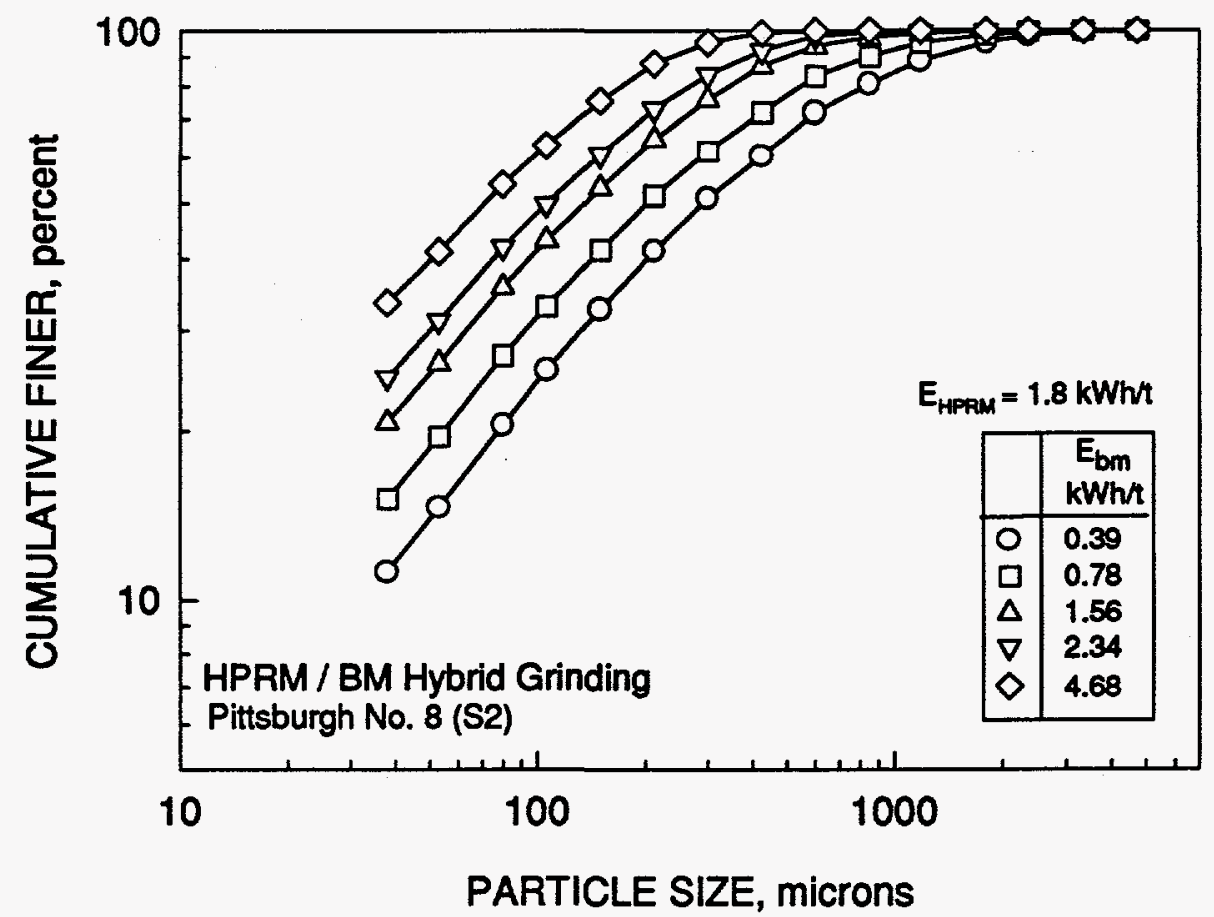

Figure 4.3. Evolution of the size distributions with increase in the ball mill grinding energy for hybrid grinding of Pittsburgh No. 8 coal, with $1.8 \mathrm{kWh} /$ tonne energy input in the high-pressure roll mill.

ball mill in order to obtain the grinding kinetics of the primary coal feed. This was to provide a baseline against which the grinding kinetics of the high-pressure roll mill products could be compared. Note that primary coal feed is equivalent to a high-pressure roll mill product formed at zero energy input.

Figure 4.3 shows typical plots of the evolution of the size spectra with increasing expenditure of grinding energy in the ball mill during the hybrid grinding of Pittsburgh No. 8 coal. Normalization of these size distributions with respect to the median size of the distribution, $\mathrm{X}_{50}$, results in a collapse of the individual distributions on to a single distribution for each coal, that is, the evolving size distributions are self-similar. The normalized size distributions are given in Figure 4.4. The size distributions obtained in the ball mill grinding of the high-pressure roll mill products, formed at various energy input levels, as well as the primary coal feed exhibit similar normalizability. 


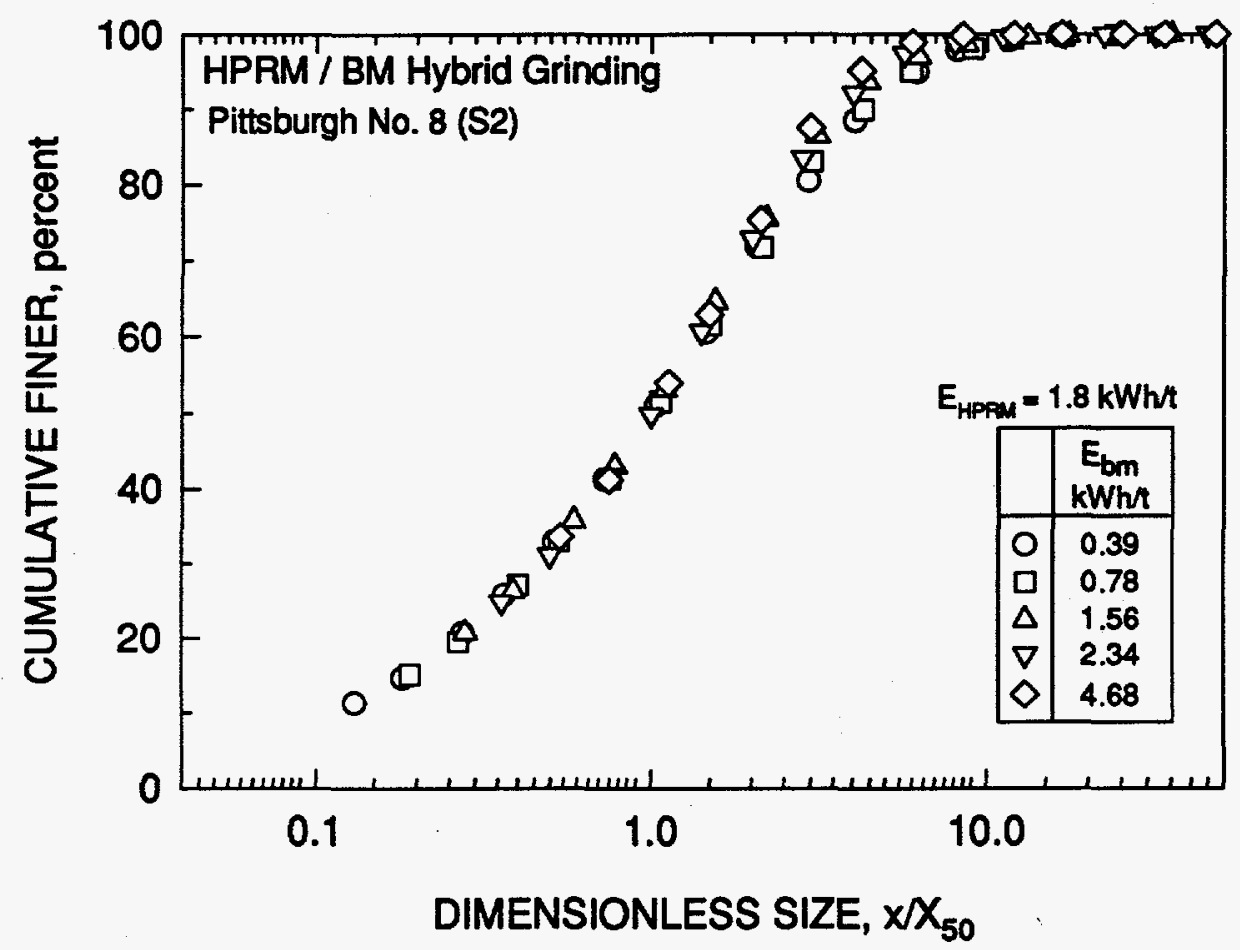

Figure 4.4. Self-preserving size spectra for the hybrid grinding of Pittsburgh No. 8 coal, with $1.8 \mathrm{kWh} /$ tonne energy input in the high-pressure roll mill.

When all the normalized distributions of the hybrid grinding products as well as those for the ball mill ground product are plotted together, we get, for all practical purposes, a single normalized size distribution, characteristic of the particular coal. It is remarkable that the self-similar size spectra resulting from hybrid grinding is identical to the one resulting from ball mill grinding of primary coal feed. This implies that the high-pressure roll mill grinding of coal only alters the particle strength without significantly altering the breakage distribution characteristics. This also means that the distribution is determined by the grinding action of the media mill only and the two modes of grinding can be compared for their energy efficiency on a completely consistent and uniform basis. The collective normalized size distributions are shown in Figure 4.5.

The self-similar distributions could be represented by the following equation :

$$
F\left(x / X_{50}\right)=1-\exp \left[-\ln 2\left(x / X_{50}\right)^{n}\right]
$$




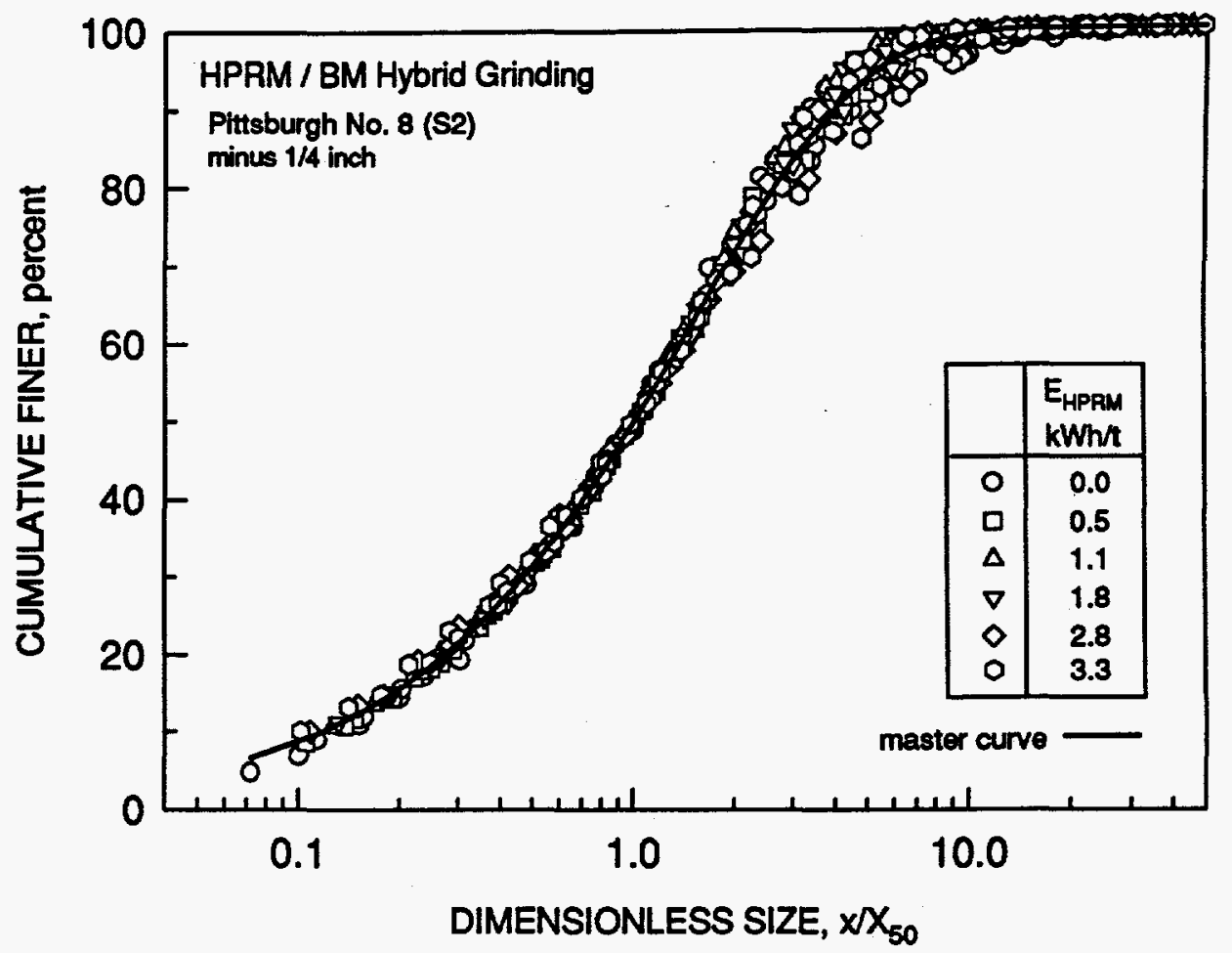

Figure 4.5. Self-preserving size spectra for the hybrid grinding of Pittsburgh No. 8 coal.

Another important aspect of the hybrid grinding of coal is the relationship between the production of minus 200-mesh fines and how the total grinding energy is split between the high-pressure roll mill and the ball mill. Because of the visco-plastic nature of coal, it is possible that the potential energy saving resulting from the fracturing and weakening of the particles under high compressive stresses could be more than offset by the significant briquetting at higher compressive stresses, corresponding to higher energy expenditures during high-pressure roll mill grinding. We could then expect to get maximum benefit from the hybrid grinding of coal at some optimal energy expenditure in the high-pressure roll mill.

Figure 4.6 presents the total energy expenditure for the production of 20,30 and $40 \%$ minus 200-mesh fines plotted against the energy expended in the high-pressure roll mill for hybrid grinding of Pittsburgh No. 8 coal. The figure indicates that there is indeed an optimum high-pressure roll mill energy corresponding to a minimum in the total energy expenditure for production of a certain percentage of fines. This is brought out even more clearly in 


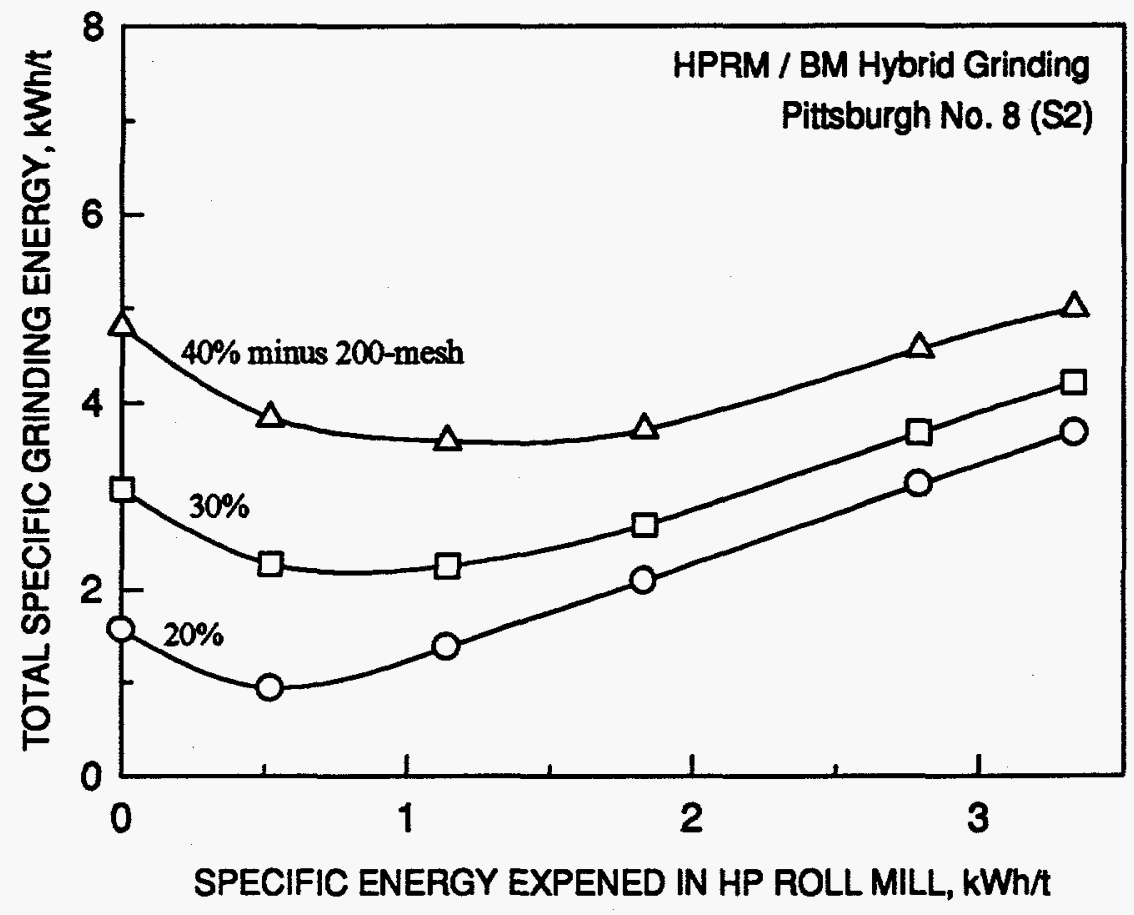

Figure 4.6. Relationship between the total specific grinding energy and the energy expenditure in the HP roll mill for the production of minus 200-mesh fines during hybrid grinding of Pittsburgh No. 8 coal.

Figure 4.7 where the percentage energy saving in the hybrid grinding mode relative to the energy expenditure in ball mill grinding alone for the production of a fixed amount of fines is plotted against the energy expended in the high-pressure roll mill. The figure clearly shows that not only there is an optimum for the energy input to the high-pressure roll mill but the energy saving is also negated if the energy expenditure in the high-pressure roll mill is higher than a threshold value; the exact value would depend on the nature of the coal and the percentage of fines produced.

\section{Hybrid Batch Locked-Cycle Grinding}

Batch grinding tests have indicated that energy savings in the order of 20 to $40 \%$ are possible for the production of minus 200-mesh coal using the open-circuit hybrid grinding mode. Because of the disproportionate increase in the grinding energy with the increase in the 


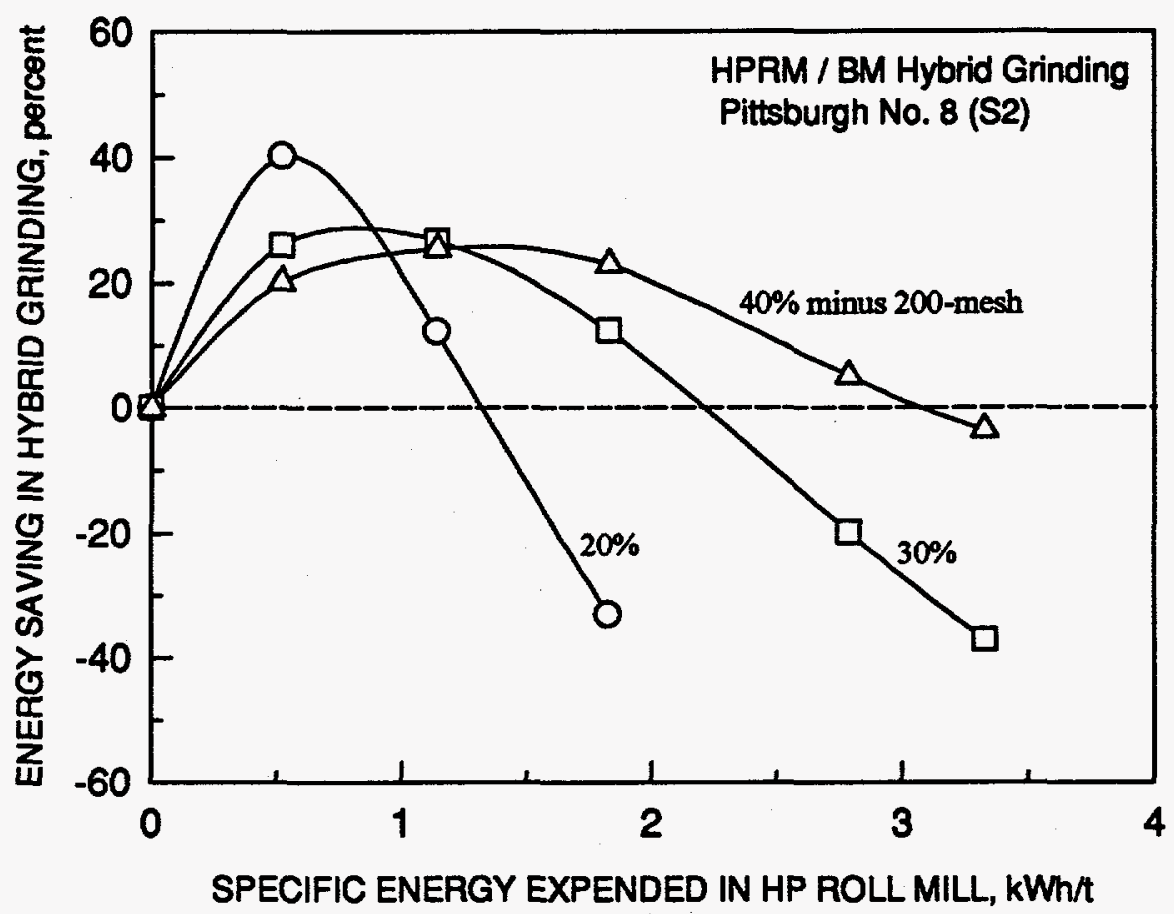

Figure 4.7. Energy saving in the hybrid grinding relative to ball mill grinding of Pittsburgh No. 8 coal, as a function of energy expended in the HP roll mill for the production of minus 200-mesh fines.

production of fines in open-loop grinding, industrial fine grinding is carried out in closed-loop grinding circuits. The projected energy savings based on open-loop hybrid grinding may not necessarily get translated directly to closed-loop grinding circuits. For obtaining more realistic estimates, closed-loop grinding tests at steady state are required, which in turn may be mimicked on the laboratory scale by locked-cycle grinding tests.

In locked-cycle grinding tests, a fixed amount of solids (either primary coal or highpressure roll mill ground product) are ground in a batch ball mill for a prescribed time duration (or energy input). The ground product is screened over the mesh-of-grind, and the oversize material is mixed with the new make-up feed for the start of the next grinding cycle. These cycles are continued until the fines produced and the recycle ratio reach steady state values, that is, they do not vary from cycle to cycle, except for limited fluctuations due to experimental errors. At steady state, the locked-cycle test is equivalent to a continuous closed-loop grinding circuit with plug flow of solids through the mill and $100 \%$ efficiency of 
the screen. The locked-cycle tests were performed i) to obtain information on the steady-state behavior of the three hybrid circuit configurations, and ii) to provide experimental data for verification of model-based simulation of locked-cycle grinding.

It had become apparent, quite early in the program, that a very large number of timeconsuming locked-cycle tests would be required to establish experimentally the optimum combination of energy inputs in the high-pressure roll mill and the ball mill for realizing the most energy-efficient two-stage hybrid grinding scheme. It was therefore decided to simulate the locked-cycle tests with a suitable mathematical model of grinding kinetics for the purpose of optimization.

Locked-cycle tests were first performed with the ball mill only for each of the coal samples in order to establish the base line values of energy consumption for the production of minus 200-mesh coal in conventional industrial grinding circuits. The locked-cycle tests were next repeated on the two-stage hybrid circuit with a loop around the ball mill.

One of the objectives of this research project was to identify the best hybrid closedcircuit grinding configuration and the optimal operating conditions which would result in minimum energy expenditure and recirculating load in the circuit. As discussed earlier, experimental determination of the optimal conditions would require a very large number of time consuming locked cycle grinding tests. On the other hand, a reasonably accurate estimation of the optimal operating conditions is possible through computer simulation of the locked-cycle grinding tests, based on a realistic mathematical model describing the kinetics of open-circuit hybrid grinding of coal.

The optimum partitioning of energy between the mills can determined either by conducting a large number of time-consuming experiments or by model-based simulation. For the simulation and optimization of the hybrid grinding circuits, we have used a highly accurate mathematical model developed to describe the kinetics of grinding of roll mill aggregates in a ball mill [36]. The salient features of the model are summarized here. 
Even though the frequently used single-value rate parameter grinding kinetic models, such as the well-known Rosin-Rammler model, can describe the kinetics of ball mill grinding of coal fairly well, these turn out to be inadequate in the case of high-pressure roll milled product. The reason seemingly lies in the highly heterogeneous nature of the briquetted feed to the ball mill, which is comprised of a mixture of primary unbroken particles and secondary particles that are broken, cracked, fissured or otherwise weakened to different extents. To account for the heterogeneity, a modified gamma function was employed to represent the distribution of the grinding rate function. In addition, in order to be able to audit and consolidate energy investments in the ball mill and the high-pressure roll mill, the kinetic equations were formulated in terms of specific energy input, rather than in terms of grinding time. It should be pointed out that there is no explicit grinding time in the roll mill - only a short time of passage through the rolls during which the taconite is subjected to a severe stress transient with a certain expenditure of energy, which is not directly related to the duration of the transient. By incorporating the gamma distribution in a generalized population balance model, the desired model of grinding kinetics in a batch ball milling mode is obtained:

$$
R\left(x, E_{b m}\right)=\left[\frac{100-F_{0}}{100}\right] \frac{\exp \left[-a_{o} x^{n} E_{b m}\right]}{\left(1+\frac{x^{n} E_{b m}}{\lambda}\right)^{\alpha}}
$$

where $R\left(x, E_{b m}\right)$ is the mass fraction coarser than size $x(\mu \mathrm{m})$ after an energy expenditure $E_{b m}$ in the ball mill, $F_{o}$ is the percentage finer than size $x$ in the feed, $a_{o}$ is the grindability of the undamaged particles, and $\alpha$ and $\lambda$ are the gamma distribution parameters.

Figure 4.8 shows the experimental and simulated size distributions for hybrid grinding of Pittsburgh No. 8 coal, where coal particles are first ground in the high-pressure roll mill at an energy expenditure of $1.83 \mathrm{kWh} / \mathrm{t}$, and then ground in the ball mill with energy investment of $0.39,0.78,1.56,2.34$, and $4.68 \mathrm{kWh} / \mathrm{t}$. As seen from the figure, the simulated distributions are in good agreement with the experimental results. 


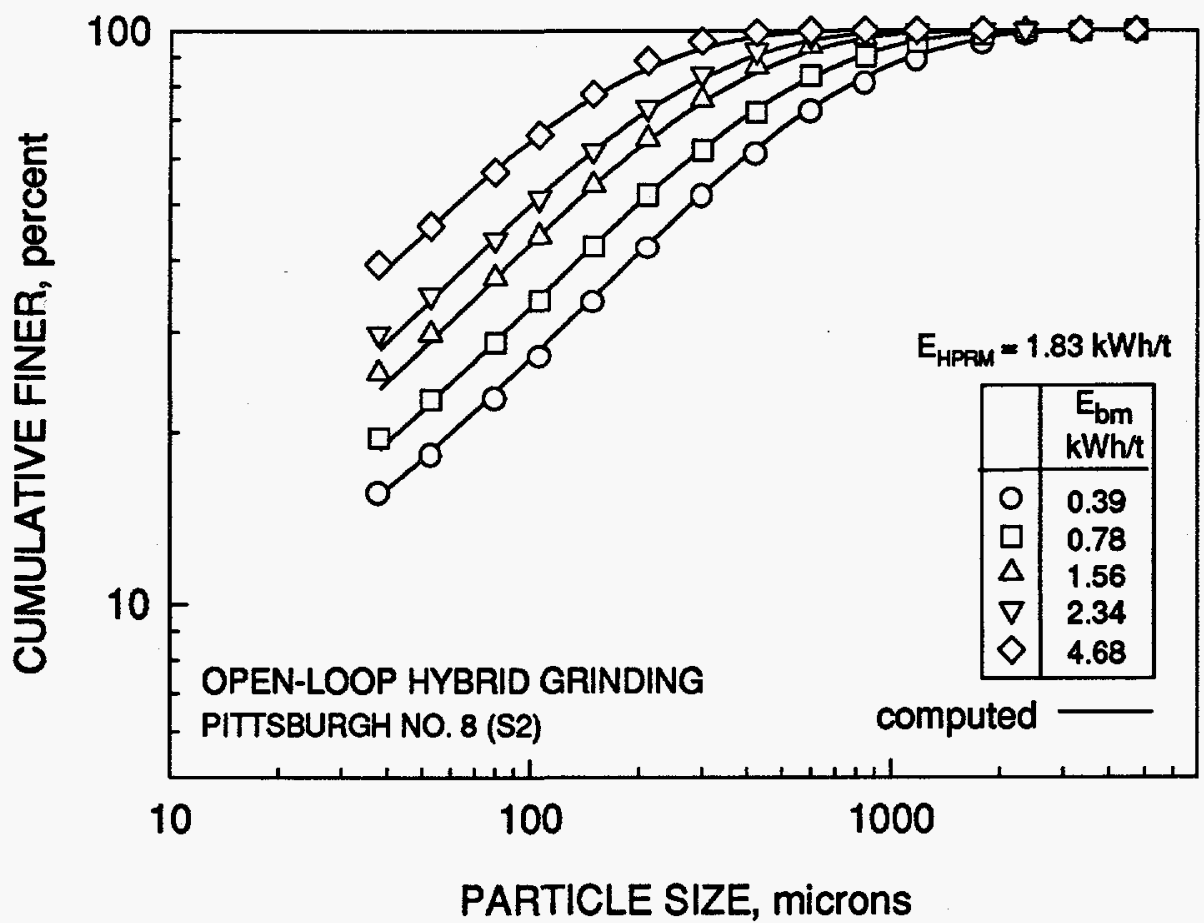

Figure 4.8. Experimental and computed size distributions of the open-circuit hybrid grinding of Pittsburgh No. 8 coal at different energy expenditures in the ball mill and 1.83 $\mathrm{kWh} / \mathrm{t}$ energy input in the high-pressure roll mill.

The model was subsequently used to simulate the production of minus 200-mesh fines in open-circuit hybrid grinding of coal over a range of energy expenditures in the two mills. The simulation studies indicated that the optimum partitioning of energy between the mills indeed results in maximum energy efficieny of comminution. The specific grinding energy ( $\mathrm{kWh} / \mathrm{t}$ of product) contours, shown in solid lines, and the contours for the percent minus 200-mesh produced, shown in dashed lines, are plotted in Figure 4.9, as functions of energy expended in the two mills. The results given in this figure clearly show that for the production of a specified amount of fines, there is an optimum partitioning of energy between the mills that results in minimum specific grinding energy for the product. For instance, for the generation of a product containing 48 percent minus 200-mesh, the maximum energy efficiency would be achieved at energy expenditures of 2.5 and $3.6 \mathrm{kWh} / \mathrm{t}$ in the high-pressure roll milling and the ball milling stages respectively. 


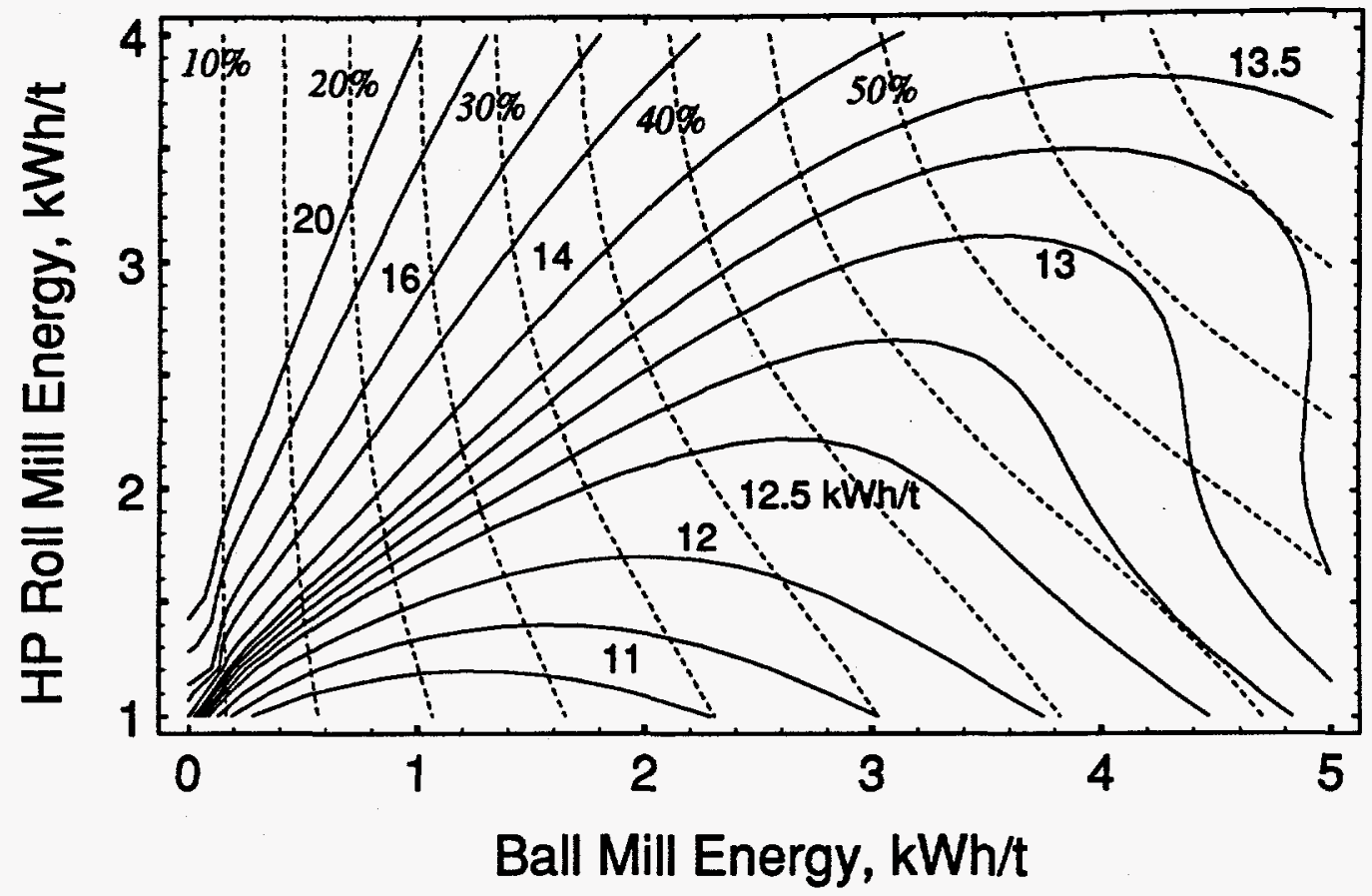

Figure 4.9. Relationship between specific grinding energy/ton of product (solid lines) and percentage of minus 200-mesh fines produced (dashed lines) at different energy inputs in the highpressure roll mill and ball mill in open-circuit hybrid grinding of Pittsburgh No. 8 coal.

The observed trends are consistent with those obtained from the experimental data and have important implications for predictive simulation of the two-stage hybrid coal grinding circuits, as discussed in the section that follows.

\section{SIMULATION OF HYBRID LOCKED-CYCLE GRINDING TESTS}

The grinding kinetics model described in the previous sub-section can be readily embedded into algorithms for cycle-wise simulation of locked-cycle grinding tests. In developing the algorithms, it was assumed that the individual feed streams could be followed cycle-wise, using the batch-grinding kinetic model

In the hybrid locked-cycle test in a ball mill loop, a fixed amount of high-pressure roll mill product is ground in the ball mill with a fixed energy input. The ball mill discharge is screened on the mesh-of-grind and the oversize is mixed with fresh high-pressure roll mill product, making up the balance of charge, and fed to the ball mill at the start of the next cycle. 
Let $\mathrm{M}$ be the total coal feed to the ball mill in any grinding cycle. After the first grinding cycle, the amount retained on the mesh-of-grind (200-mesh in present case) and recycled back as feed for second grinding cycle is $M R\left(E_{b m}\right)$ and the new make-up feed is $M-M R\left(E_{b m}\right)$. The amount retained on the mesh-of-grind and recycled back after the second cycle is:

$$
R c(2)=M R\left(2 E_{b m}\right)+\left[M-M R\left(E_{b m}\right)\right] R\left(E_{b m}\right)
$$

and the amount of minus 200-mesh fines produced is:

$$
F(2)=M-R c(2)
$$

At the end of the third cycle, the amount recycled back as feed is:

$$
R c(3)=M R\left(3 E_{b m}\right)+\left[M-M R\left(E_{b m}\right)\right] R\left(2 E_{b m}\right)+[M-R c(2)] R\left(E_{b m}\right)
$$

and the amount of fines produced is:

$$
\mathrm{F}(3)=\mathrm{M}-\mathrm{Rc}(3)
$$

where $R(x E) ; x=1,2,3, .$. is given by Equation 4.3. The cycle-wise computations are continued until the recycle and fines produced become sensibly constant from cycle to cycle, that is, a steady-state is reached.

Figure 4.10 compares the simulated locked-cycle test results with the experimental data for Pittsburgh No. 8 coal. Overall, quite good agreement was obtained, which suggested that the locked-cycle grinding test algorithm could be used to identify the optimum partition of grinding energies in the high-pressure roll mill and the ball mill.

\section{OPTIMIZATION OF THE HYBRID GRINDING CIRCUITS}

The enhanced efficiency of hybrid grinding results from the internal weakening or damage to the particles that the HP roll mill particle bed grinding imparts. The ball mill grinding step of the hybrid mode takes advantage of this damage, producing a finer material after comparable energy input than either of the two devices alone. However, correct partitioning of the grinding energy between the HP roll mill and the ball mill (or any other such comminution device used for disintegration of the briquettes) is necessary to achieve maximum energy savings. 


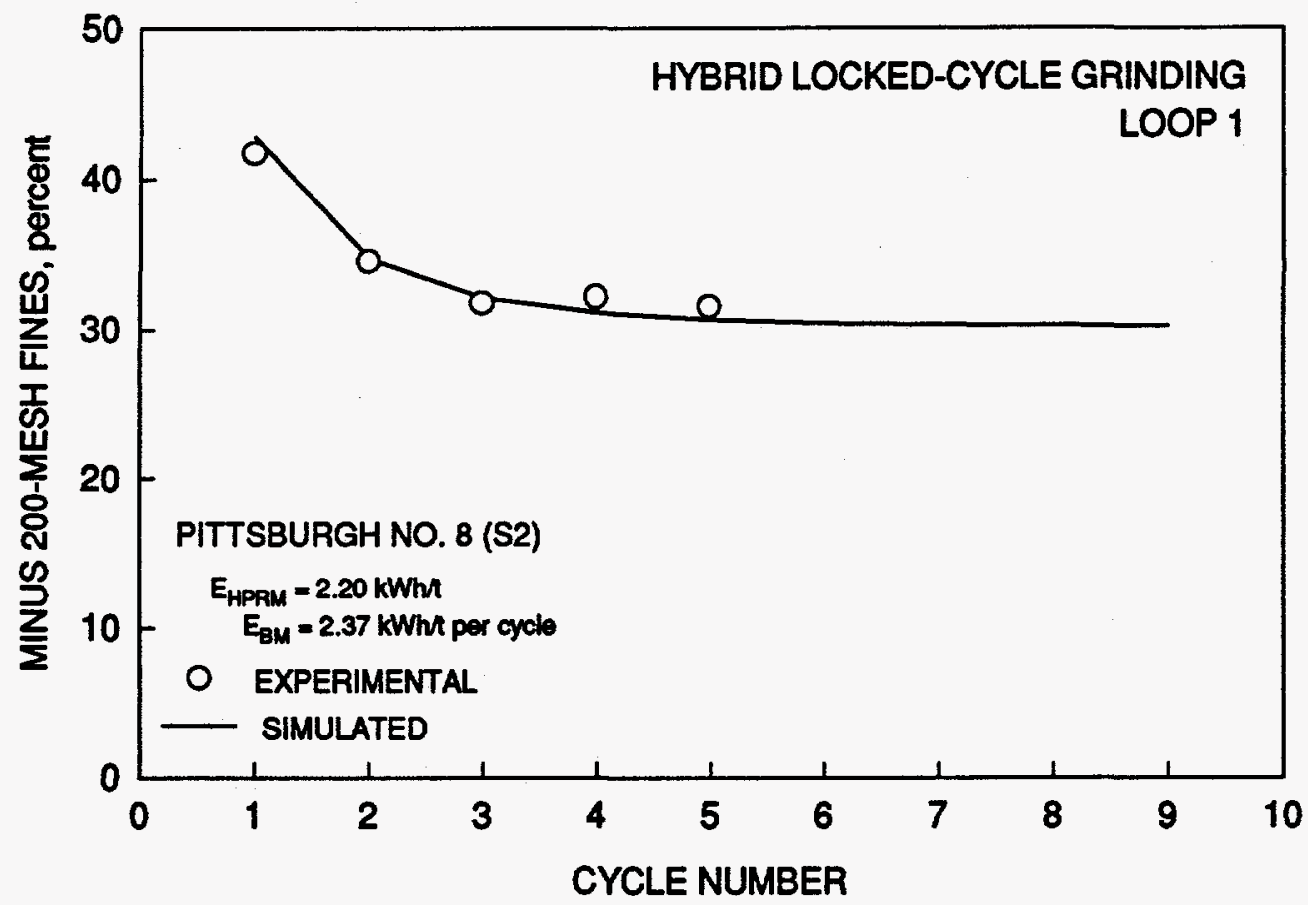

Figure 4.10. Experimental results and simulation curves for the locked-cycle hybrid grinding of Pittsburgh No. 8 coal, with oversize recycle to the ball mill and energy inputs of 2.2 $\mathrm{kWh} / \mathrm{t}$ in the high-pressure roll mill and $2.37 \mathrm{kWh} / \mathrm{t}$ per cycle in the ball mill.

The optimal operating conditions for a closed-loop grinding circuit is dictated primarily by two conflicting objectives: maximum throughput of the product at minimum specific energy expenditure. There are additional constraints, like mill size and recirculating load, which directly influence the throughput. The closed-loop hybrid grinding circuits modeled in the previous sections have two degrees of freedom: the energy inputs in the highpressure roll mill and in the ball mill. Optimization of these circuits would therefore involve finding the optimal energy expenditure in the two mills which would satisfy the objectives and the constraints mentioned above. Moreover, the experimental data as well as the simulation results on the hybrid batch grinding kinetics have shown that an optimum partition exists between the energy input in the high-pressure roll mill and the ball mill in order to get a maximum percentage of minus 200-mesh product. Similar trends are expected in closedcircuit grinding as well. The simulation studies presented in this section were aimed at determining the optimal grinding energy expenditure and the partitioning of the energy 
between the high-pressure roll mill and the ball mill.

The locked-cycle grinding equation derived earlier can be written in a more compact form, with unit feed rate as the basis, as follows:

$$
P_{i}=1-\sum_{j=1}^{i} F_{j} \Phi_{i-j+1} ; \quad F_{1}=1 ; \quad F_{i+1}=P_{i}
$$

where $F_{i-j+1}$ represents the fraction of the feed stream $F_{j}$ remaining in the oversize after $(i-j+1)$ pass through the circuit. $P_{i}$ is the minus 200 -mesh produced in the $i^{\text {th }}$ cycle. For Loop $1, F$ for the $\mathrm{c}^{\text {th }}$ pass is given by:

$$
\Phi_{c}=\frac{\left(1-F_{200}\right) \exp \left(-c k_{o} E_{b m}\right)}{\left(1+c E_{b m} / \lambda\right)^{\alpha}}
$$

If $\mathbf{P}_{\mathbf{S}}$ is the fines produced (as fraction of feed) per cycle at steady-state, when the specific energy input per pass in the high-pressure roll mill is $E_{h p}$ and the specific energy expended per cycle in the ball mill is $\mathrm{E}_{\mathrm{bm}}$, then the overall energy consumption per unit mass of fines produced is:

$$
E_{200}=E_{b m} / P_{s}+E_{h p}
$$

Equations 4.8 and 4.9 were used to simulate the steady state production of minus 200mesh fines and the specific energy required to produce one ton of minus 200-mesh fines as a function of the energy input to the high-pressure roll mill and the ball mill in hybrid lockedcycle grinding. Figure 4.11 shows the simulated minus 200 -mesh coal fines produced per cycle at steady-state, as a function of the energy inputs in the high-pressure roll mill and the ball mill for grinding of Pittsburgh No. 8 coal. Figure 4.12 presents the overall specific energy consumption at steady state required to produce the minus 200-mesh fines in hybrid closedcircuit grinding. As shown in these figures, while the percentage fines produced increases monotonically with increase in the energy inputs to either of the mills, optimal partitioning of energy between the mills could result in minimum specific energy consumption for the production of fines. 


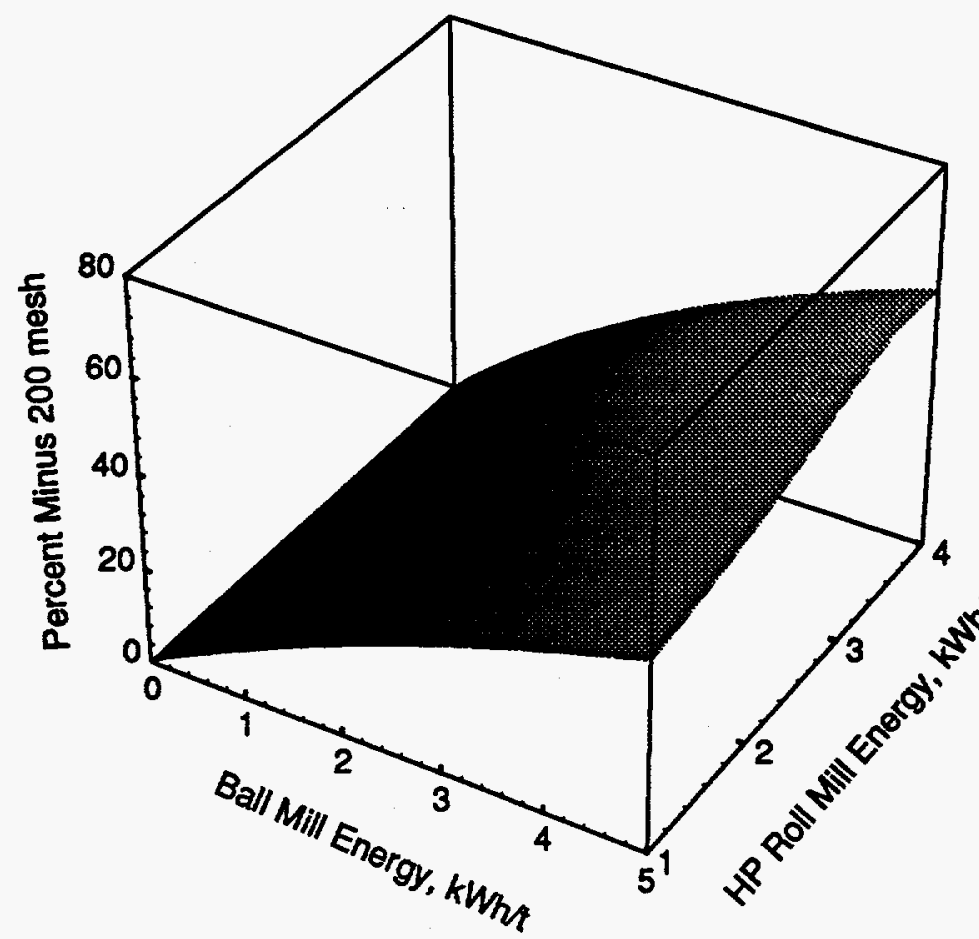

Figure 4.11. Minus 200-mesh fines produced at steady-state as a function of the energy inputs in the high-pressure roll mill and the ball mill for closed-circuit hybrid grinding of Pittsburgh No. 8 coal.

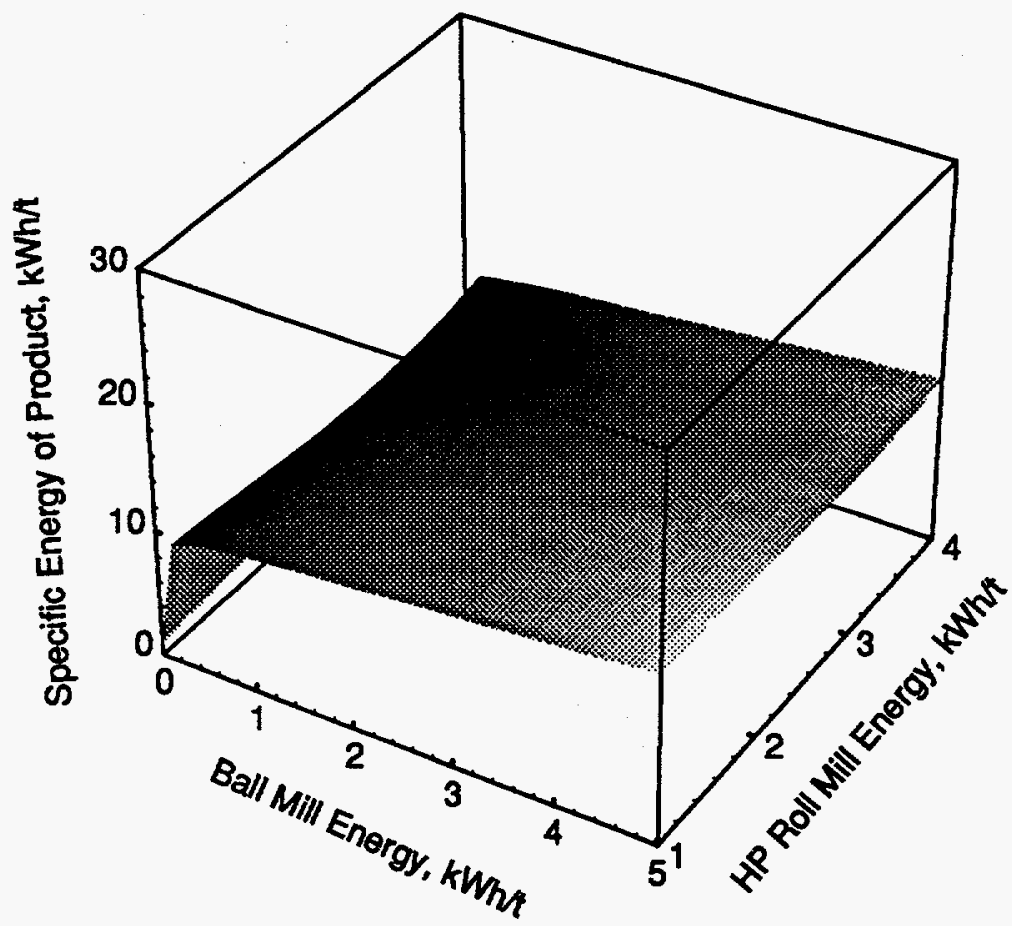

Figure 4.12. Specific energy consumption, for production of minus 200 -mesh fines, at steady-state as function of the energy inputs in the high-pressure roll mill and the ball mill for closedcircuit hybrid grinding of Pittsburgh No. 8 coal. 


\section{EFFECT OF HIGH-PRESSURE ROLL MILL GRINDING OF COAL ON THE SLURRY RHEOLOGY}

So far, we have shown that under optimal operating conditions both open- and closed-circuit hybrid grinding of coal could result in signifucant savings in grinding energy as compared to grinding coal in a ball mill alone. We now present the results of comminution experiments carried out that were designed to delineate the effect of high-pressure roll mill grinding of coal on the rheology of coal-water slurries prepared using fines produced by subsequent ball milling of the high-pressure roll mill product.

Minus 8-mesh Pittsburgh No. 8 feed was first ground in the laboratory high-pressure roll mill at applied loads of 2.0,3.7 and 9.0 tons, which correspond to energy expenditures of $0.69,1.17$ and $2.86 \mathrm{kWh} / \mathrm{t}$, respectively. The roll mill product was then ground in an 8-inch stainless steel ball mill in both open- and closed-circuit modes. In the case of open-circuit grinding, the roll mill product was ground in the ball mill for various lengths of time, after which the mill discharge was dry-sieved on a 200-mesh screen using a Ro-Tap machine. The minus 200-mesh product was used to prepare our coal-water slurries. In closed-circuit tests, the high-pressure roll mill product was ground in the ball mill for a fixed length of time, after which the mill discharge was dry-screened at 200 mesh, with the minus 200-mesh material being the desired product. The screen oversize is mixed with fresh high-pressure roll mill product to make up the balance of charge to the ball mill for the next grinding cycle.

In addition, minus 8-mesh feed was ground in the ball-mill alone and in both the openand closed-circuit modes for the production of minus 200-mesh fines. The rheological characteristics of the slurries prepared from this material provide the baseline against which behavior of the slurries prepared from fines produced by the hybrid high-pressure roll mill / ball mill grinding of coal could be compared. Table 4.1 shows the percentage minus 200-mesh produced by ball milling of three high-pressure roll mill products and the minus 8mesh feed in the open-circuit mode for different ball milling times. The results indicate that the high-pressure roll mill product grinds considerably faster than the primary feed in the ball mill. 
Table 4.1. Rate of production of minus 200-mesh fines in open-circuit ball milling of roll mill products, produced with various energy inputs in the high-pressure roll mill.

\begin{tabular}{|c|cccc|}
\hline & \multicolumn{4}{|c|}{ Energy expended in the high-pressure roll mill, $\mathrm{kWh} / \mathrm{t}$} \\
\multirow{2}{*}{$\begin{array}{l}\text { Grinding time in } \\
\text { the ball mill, } \\
\text { min. }\end{array}$} & 0 (ball mill only) & 0.69 & 1.17 & 2.86 \\
\cline { 2 - 5 } & \multicolumn{4}{|c|}{ percent minus 200 mesh produced } \\
\hline 1 & & 18.0 & 20.0 & 23.4 \\
2 & 16.5 & 24.8 & 26.0 & 32.0 \\
4 & 29.5 & 36.0 & 36.5 & 44.0 \\
8 & 46 & & & \\
\hline
\end{tabular}

In particular, an energy expenditure of $2.9 \mathrm{kWh} / \mathrm{t}$ in the high-pressure roll mill results in a product that grinds twice as fast as the primary feed in the ball mill.

The size distributions of the various minus 200-mesh samples were determined using the L\&N Microtrac Particle-Size Analyzer. Figure 4.13a gives the size distributions of the various feeds used for making the coal-water slurries; in Figure 4.13b the size distributions are replotted in dimensionless size, that is, with size rescaled by the median size. The collapse of the distributions on to a single curve indicates that both open- and closed-circuit grinding configurations result in similar particle size distributions.

The minus 200-mesh product from the open-circuit hybrid grinding tests were used to prepare coal-water slurries containing 67 wt\% solids. CoalMaster A23-M was added as dispersant in each of these slurries, the dosage being $1 \mathrm{wt} \%$ based on the solids. Figure 4.14 shows the flow curves for slurries prepared with fines produced by the ball mill grinding of high-pressure roll mill products, produced at an energy expenditure of $1.2 \mathrm{kWh} / \mathrm{t}$, for different lengths of time. The flow curves of slurries prepared with fines produced by ball milling of the three high-pressure roll mill products, for two minutes in the open-circuit mode, are compared in Figure 4.15. As can be seen from the plots given in Figure 4.14, these slurries behave like Newtonian fluids with a small yield. The small yield probably indicates a low 

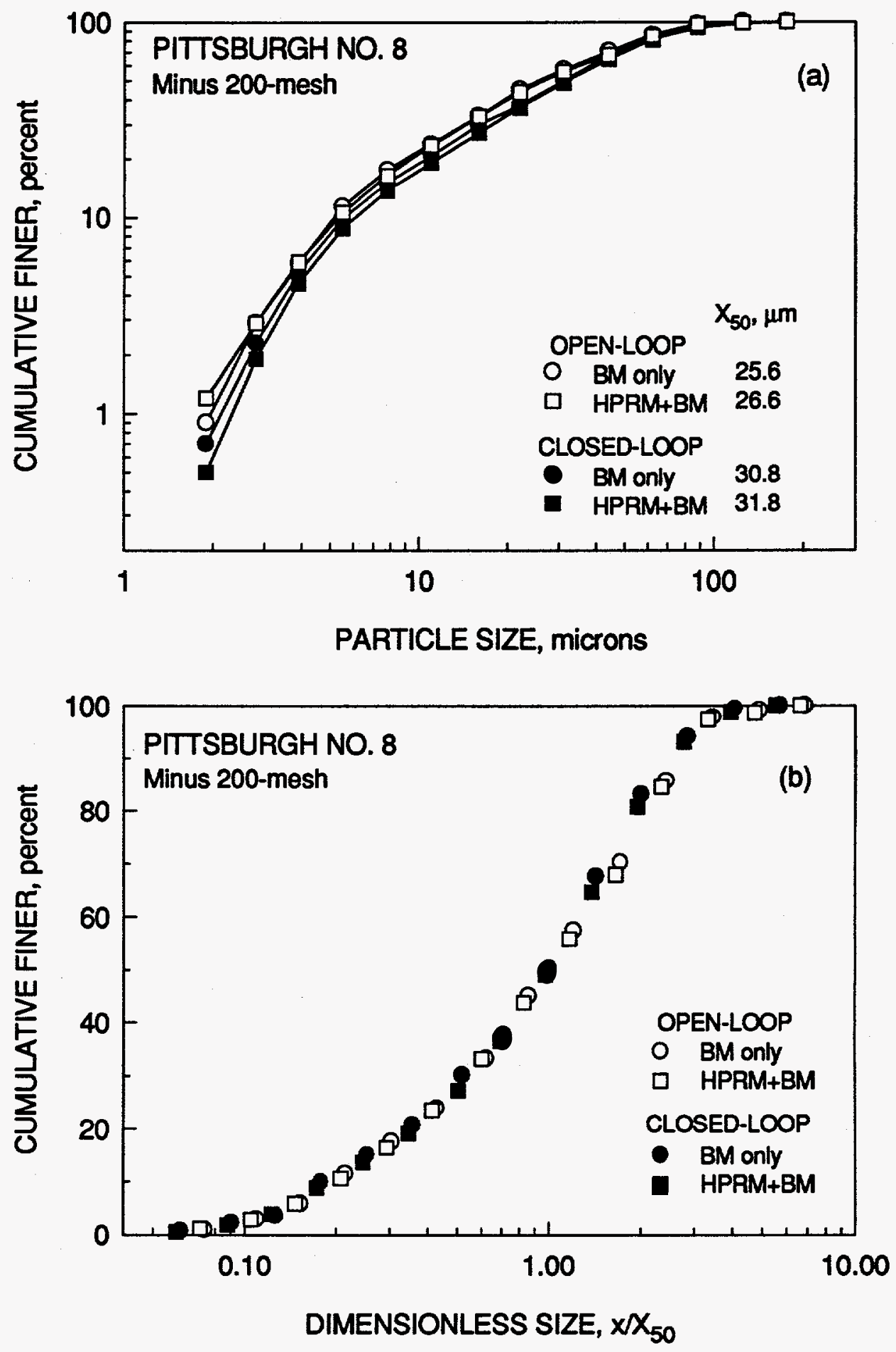

Figure 4.13. (a) Size distributions of the minus 200-mesh products obtained with the different modes of grinding, and (b) the same distributions rescaled in terms of dimensionless size. 

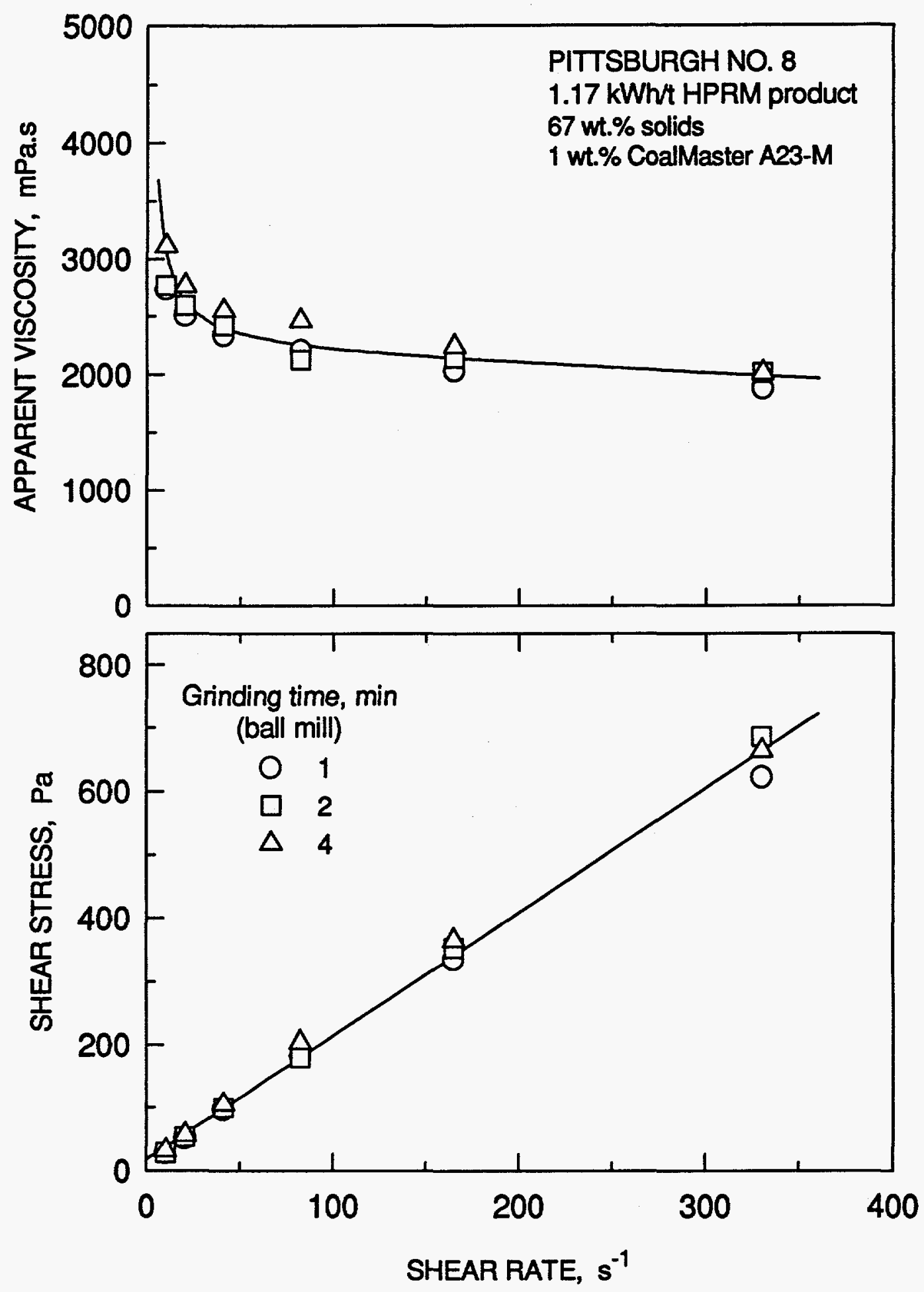

Figure 4.14. The effect of ball mill grind time on the viscosity and shear stress, measured as a function of shear rate, of slurries prepared with fines produced by open-loop ball milling of $1.17 \mathrm{kWh} / \mathrm{t}$ high-pressure roll mill product. 

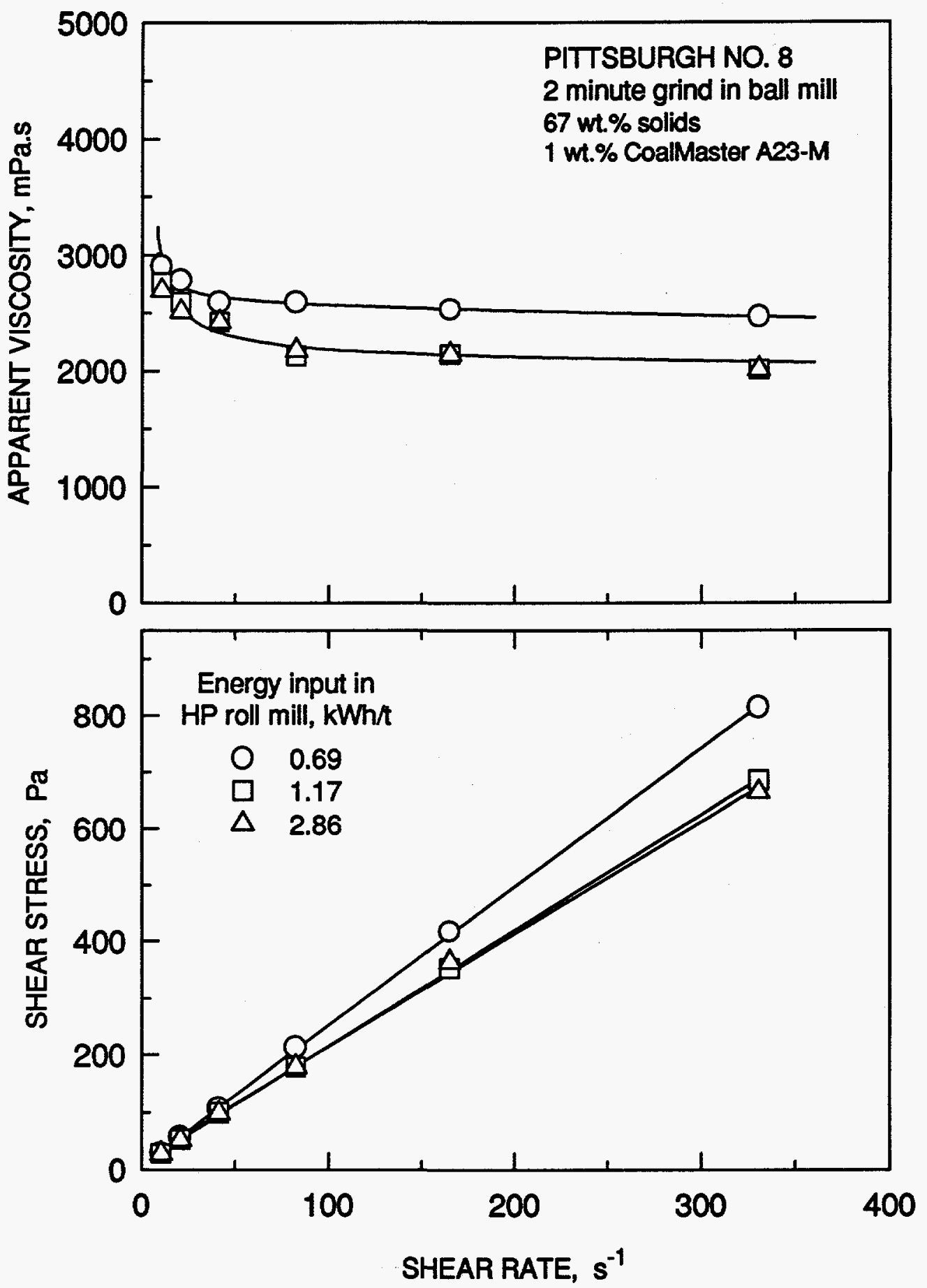

Figure 4.15. The effect of energy input in the high-pressure roll mill on the viscosity and shear stress, measured as a function of shear rate, of slurries prepared with fines produced by open-loop ball milling of high-pressure roll mill products for 2 minutes. 
soluble iron content of the fines. The fact that these slurries have practically identical flow curves suggests that the time of grind in the ball mill does not significantly alter the characteristics of the fines. It is apparent from Figure 4.15, however, that the increase in energy expended in the high-pressure roll mill results in a slight decrease in the viscosity of the slurries.

Locked-cycle grinding tests were performed with each of the high-pressure roll mill products, with 2 minutes grind time in the ball mill per grinding cycle. An additional lockedcycle test was carried out with the minus 8-mesh feed and a 4-minute grind time per cycle. The percentages of minus 200-mesh produced at steady state were 29, 20, 22 and 25 for the feed and the $0.69-\mathrm{kWh} / \mathrm{t}, 1.17-\mathrm{kWh} / \mathrm{t}$ and $2.86-\mathrm{kWh} / \mathrm{t}$ high-pressure roll mill products, respectively. Figure 4.16 shows the flow curves of slurries prepared with fines produced at steady state in these locked-cycle grinding tests. The beneficial effect of high-pressure roll milling on the rheology of coal-water slurries is clearly discernible from the results given in this figure. The apparent viscosity of these slurries are plotted as a function of the shear rate in Figure 4.17. As can be seen from the figure, an increase in the energy expenditure in the high-pressure roll mill not only results in lowering of viscosity but also the fineness of the minus 200-mesh product. In general, the inverse relationship between the energy expenditure in the high-pressure roll mill and the viscosity, observed earlier for slurries produced with fines generated by open-circuit hybrid grinding, holds for slurries prepared with fines produced by closed-circuit hybrid grinding.

\section{INFLUENCE OF GRINDING ENVIRONMENT IN THE BALL MILL ON THE ENERGY UTILIZATION AND THE RHEOLOGY OF SLURRIES}

Fine grinding of coal for our research has been carried out mostly under dry conditions primarily because of the difficulty of experimentation using wet grinding conditions. Preparation of coal-water slurries with dry fines involves addition of water and 

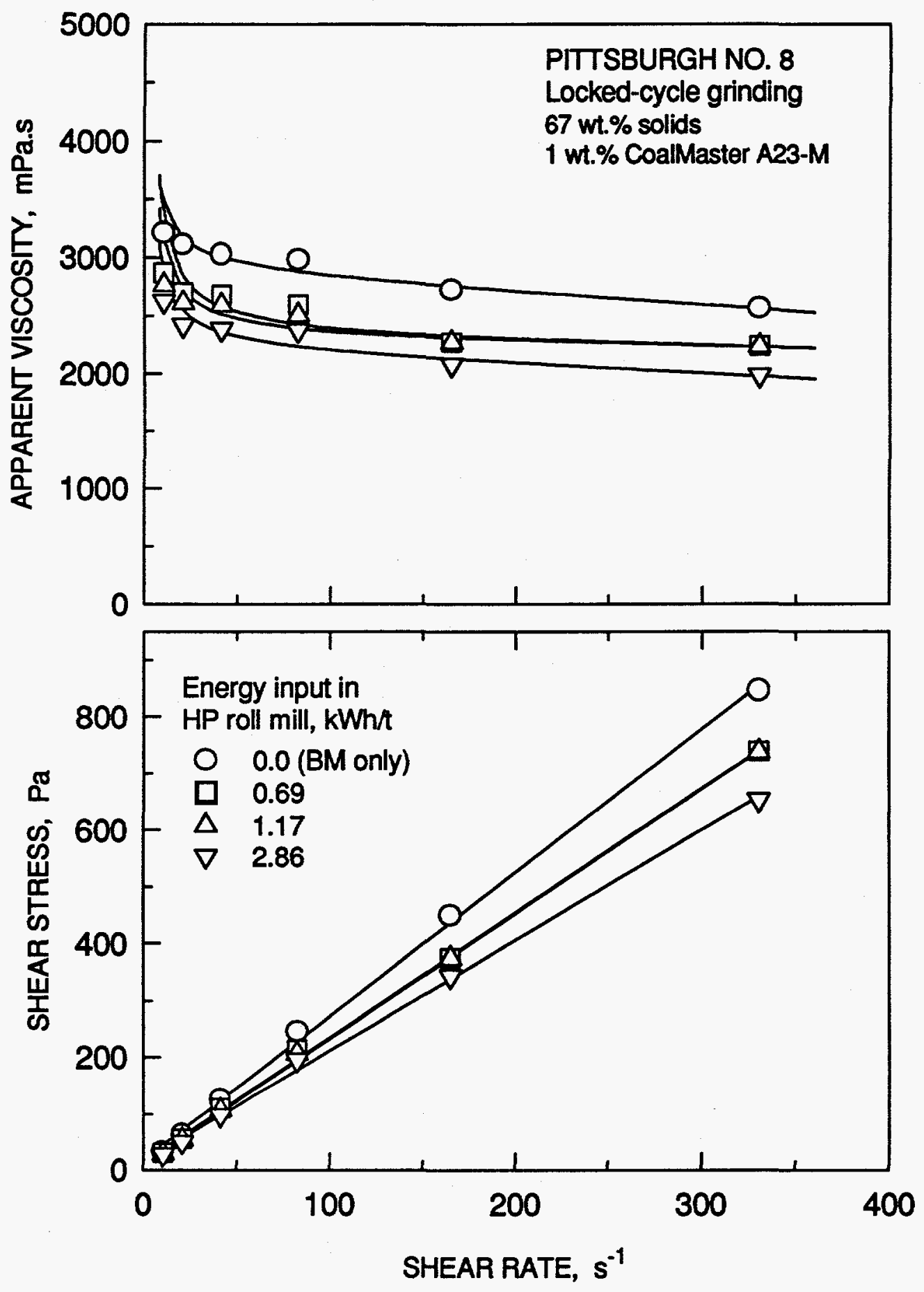

Figure 4.16. The effect of energy input in the high-pressure roll mill on the viscosity and shear stress, measured as a function of shear rate, of slurries prepared with fines produced by closed-loop ball milling of various high-pressure roll mill products. 


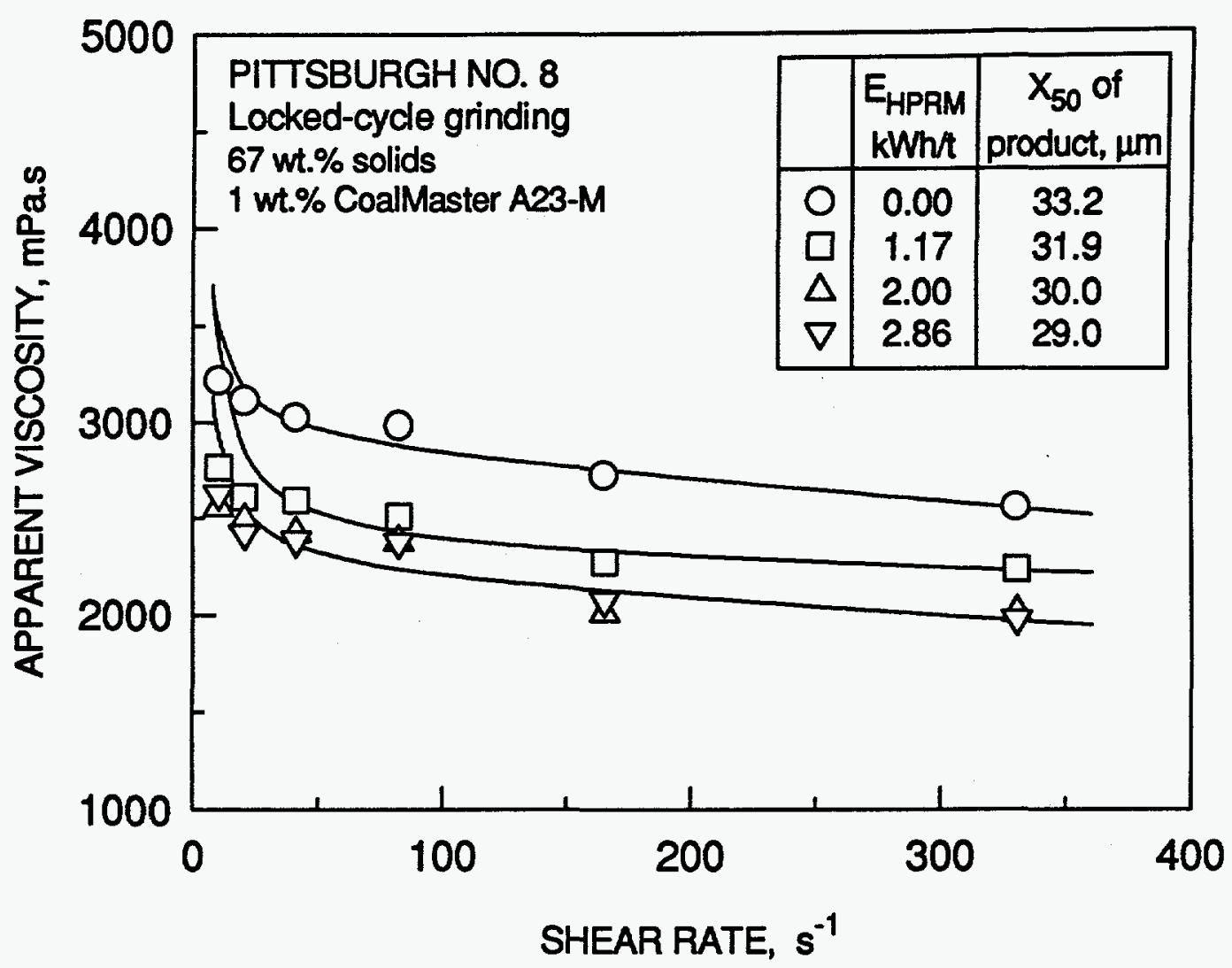

Figure 4.17. The effect of energy input in the high-pressure roll mill on the apparent viscosity, measured as a function of shear rate, of slurries prepared with fines produced by closed-loop ball milling of various high-pressure roll mill products.

mixing at high shear. It is known that energy utilization in wet grinding is slightly higher than that in dry grinding, Optimal energy utilization in wet grinding, however, depends on the solids content of the slurry in the mill. Beyond a certain solids content, there is a significant decrease in the energy utilization with increasing fineness of grind. The addition of grinding aids which lower the viscosity of the slurry helps prevent this loss in efficiency. In the mineral processing industry, reagents are often added to the wet-grinding stage to facilitate uniform adsorption of reagents on the mineral surfaces. A series of experiments were therefore performed to study the effect of wet grinding with and without dispersant on the energy utilization and rheology of slurries prepared with fines produced by wet grinding. 


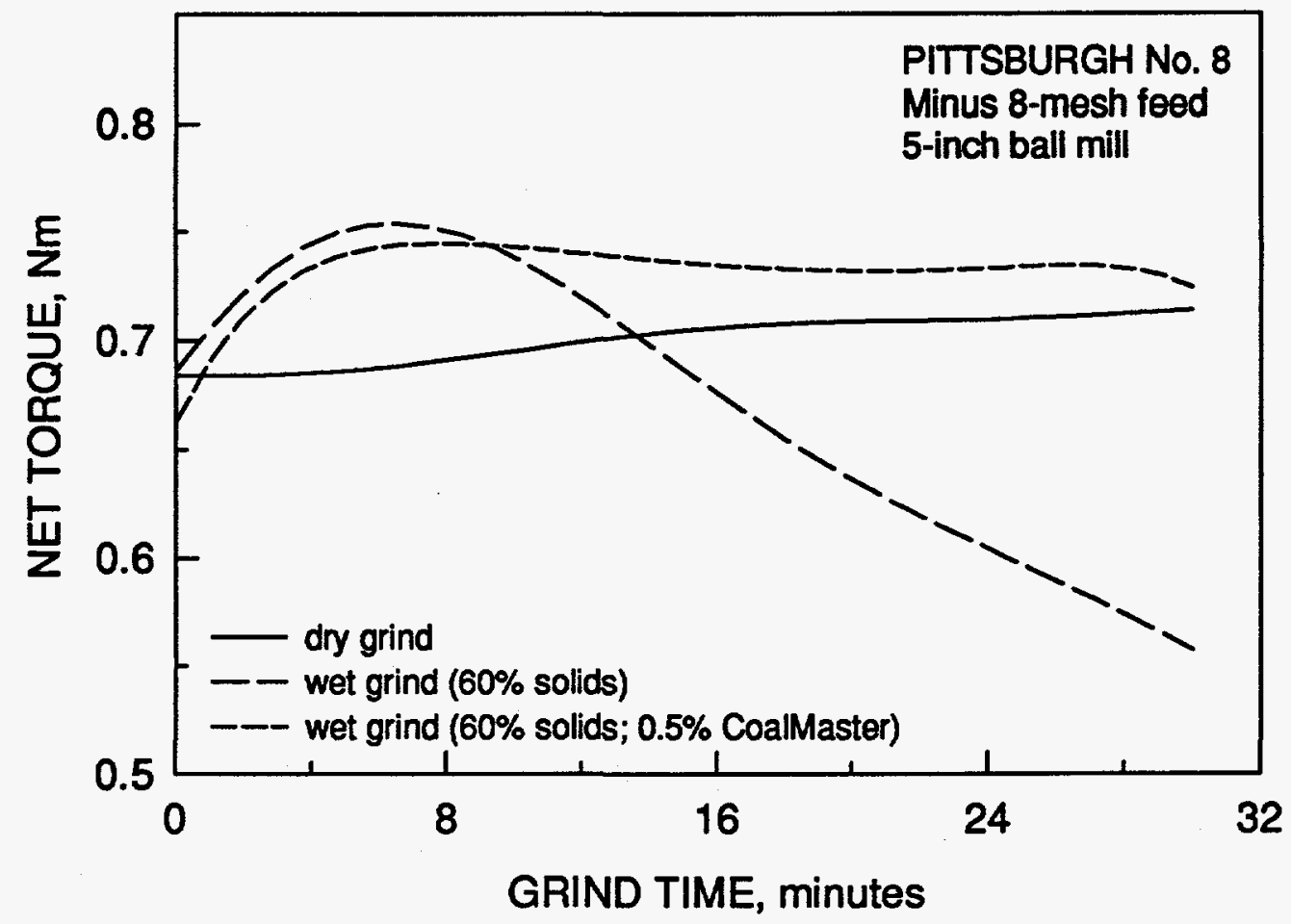

Figure 4.18. Effect of grinding conditions on the efficiency of ball mill grinding of primary feed.

A minus 8-mesh coal sample was ground in the 5-inch torque ball mill for 30 minutes under dry, wet, and wet with an addition of $0.5 \mathrm{wt} \%$ CoalMaster. Wet grinding was carried out at $60 \mathrm{wt} \%$ solids content. The torque and the mill speed were continuously measured during the grinding experiments. Figure 4.18 shows the variation of net torque with time for each of these experiments. While the torque during dry grinding remains more or less constant with time, there is a steady decrease in the grinding torque with time beyond the first few minutes. As can be seen from the figure, the addition of CoalMaster prevents such a lowering of torque.

Similar experiments were carried out with high-pressure roll mill ground product. The torque-time profiles for these experiments are given in Figure 4.19. Unlike the results for wet grinding of primary feed without CoalMaster, there is no decrease in torque over time for wet grinding of the high-pressure roll mill product. This is perhaps due to improved packing density of the feed during high-pressure roll mill grinding. 
Table 4.2. Effect of grinding conditions on the efficiency of ball mill grinding of primary feed and high-pressure roll mill product.

\begin{tabular}{|l|c|c|c|c|}
\hline \multicolumn{1}{|c|}{ Grinding condition } & \multirow{2}{*}{$\begin{array}{c}\text { Sp. grinding } \\
\text { energy, } \mathrm{kWh} / \mathrm{t}\end{array}$} & $\begin{array}{c}\text { \% minus } \\
200 \text {-mesh }\end{array}$ & \multicolumn{2}{|c|}{ Median size, $\mu \mathrm{m}$} \\
\cline { 4 - 5 } & & & Mill discharge & Minus 200-mesh \\
\hline Primary feed & 15.0 & 85.2 & 33.9 & \\
Dry & 14.5 & 87.0 & 32.3 & 27.3 \\
Wet (60\% solids) & 15.9 & 92.1 & 28.4 & 24.0 \\
Wet (60\% solids) with & & & & \\
0.5 wt\% CoalMaster & & & & \\
HPRM product & 14.7 & 90.9 & 29.2 & 26.0 \\
Dry & 15.6 & 94.2 & 24.8 & 2.3 \\
Wet (60\% solids) & 14.6 & 92.3 & 26.2 & 2.5 \\
Wet (60\% solids) with & & & & \\
0.5 wt\% CoalMaster & & & & \\
\hline
\end{tabular}

Table 4.2 gives a summary of the grinding conditions for each of these experiments as well as the total specific grinding energy and median sizes of the ball mill discharge and the minus 200-mesh fraction in the discharge. Although there are differences in the specific grinding energy and the product median size, the particle size distributions are esse ntially selfsimilar as shown in Figure 4.20.

Besides improving the efficiency of grinding, wet grinding probably results in better rheological properties, as suggested by the viscosity measurements on slurries prepared with minus 200-mesh fines produced by wet grinding. Figure 4.21 shows the apparent viscosity plots as a function of shear rate for the slurries prepared with fines produced by ball mill grinding of primary feed under different conditions as well as by wet grinding of the highpressure roll mill product without any dispersant. If the effect of median size of the distribution is taken into account, the results presented in the figure indicate that wet grinding results in a lowering of viscosity. The beneficial effect of high-pressure roll milling is also observable. 


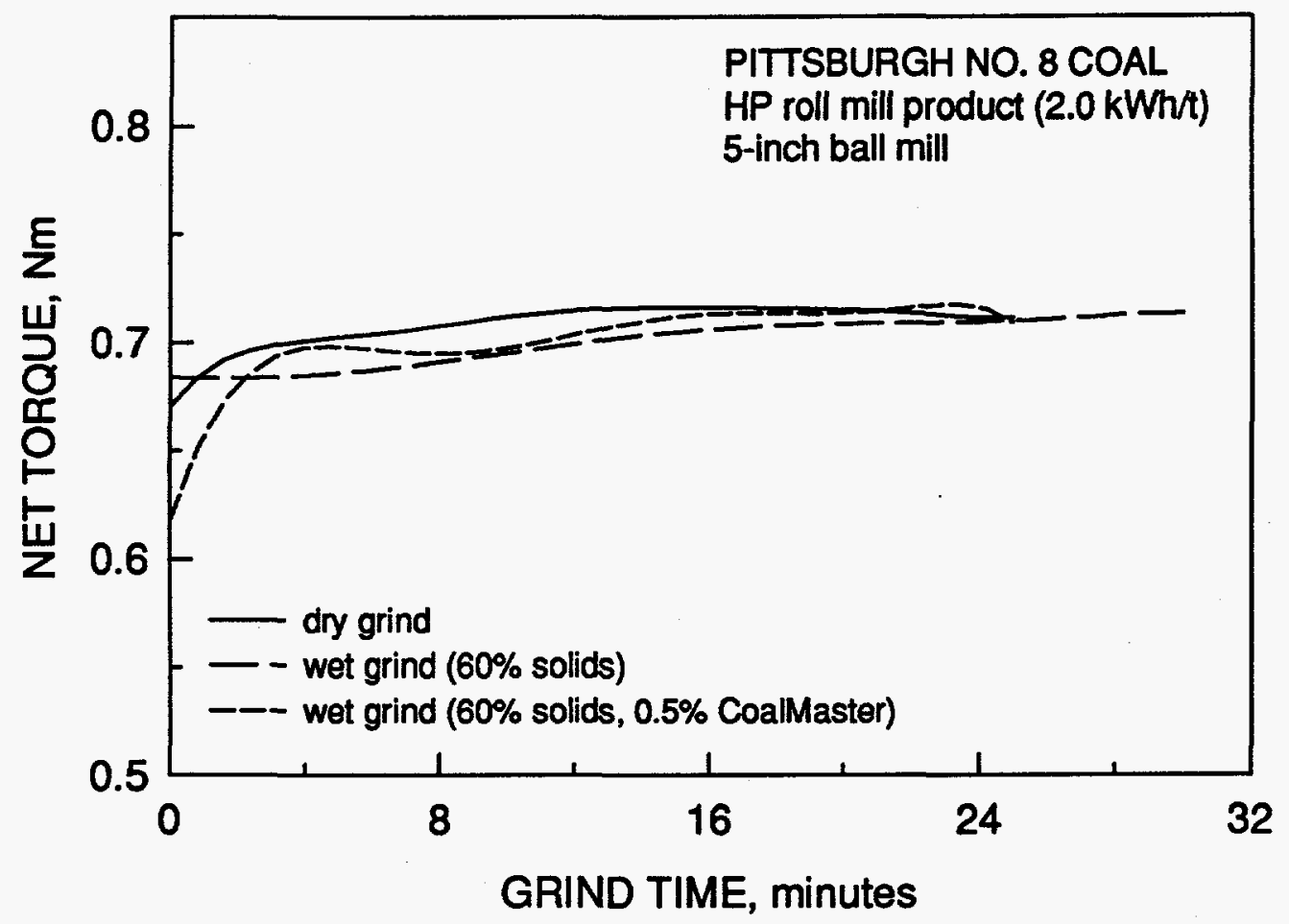

Figure 4.19. Effect of grinding conditions on the efficiency of ball mill grinding of high-pressure roll mill product.

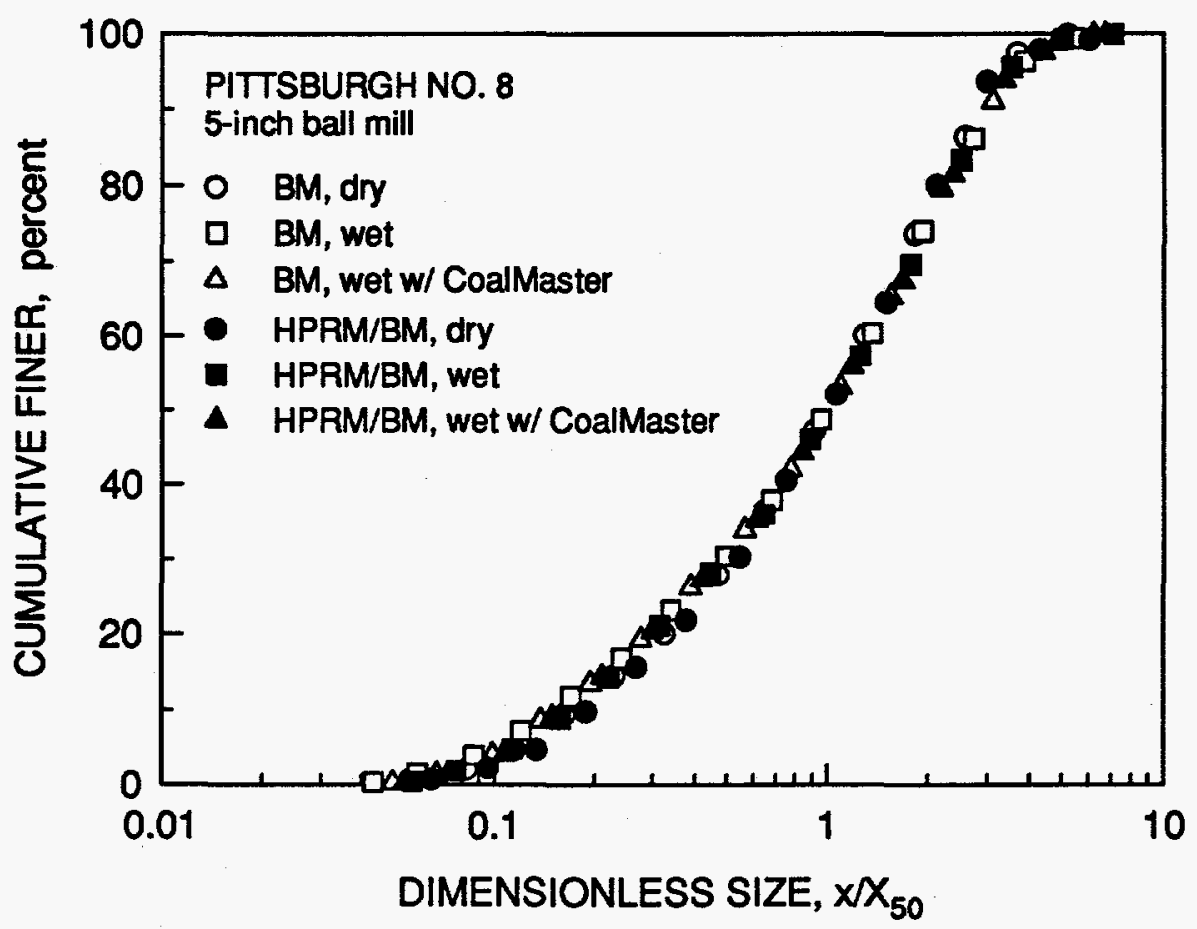

Figure 4.20. Self-similarity of size distributions produced by ball milling of primary feed as well as high-pressure roll mill product under different grinding conditions. 


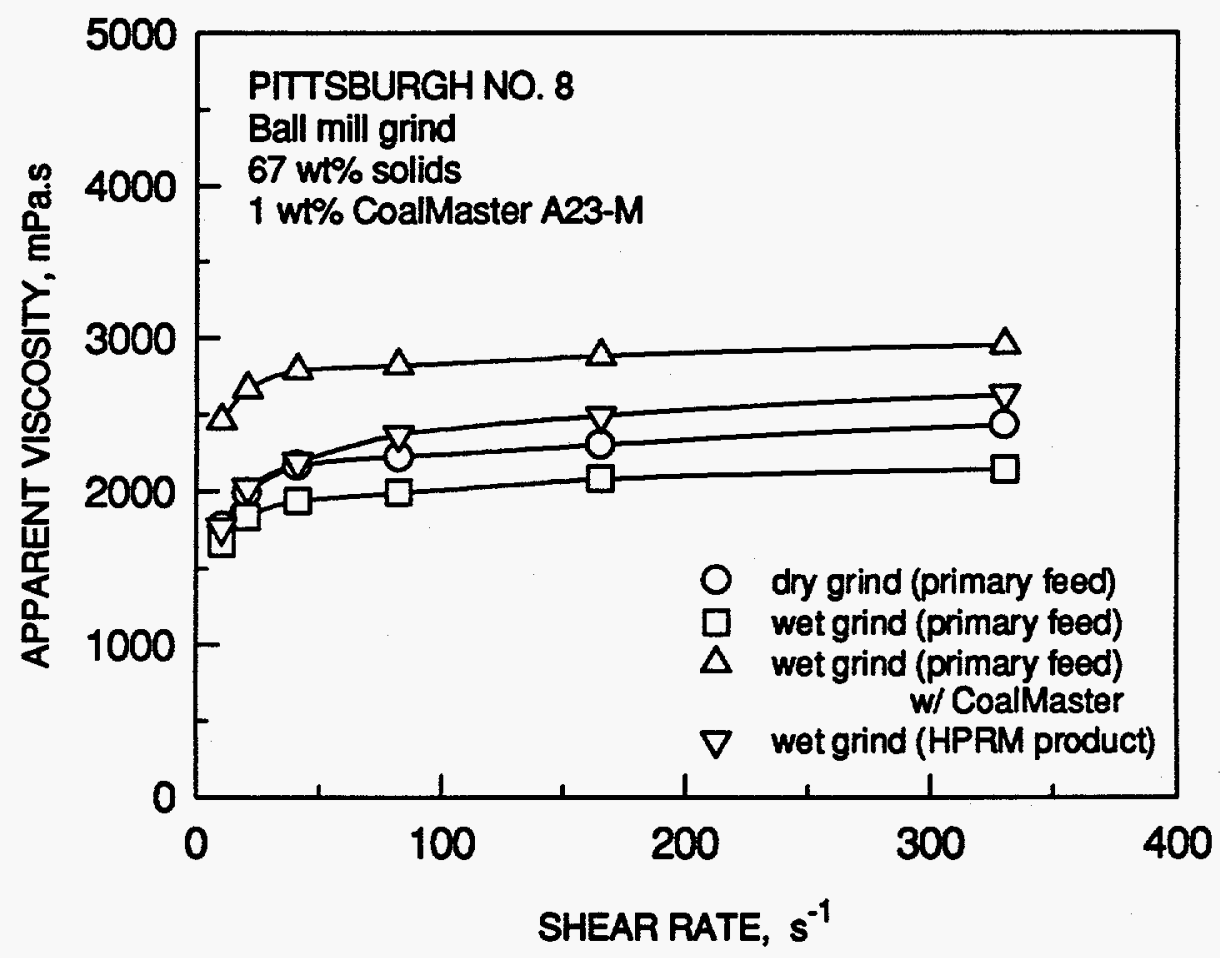

Figure 4.21. Apparent viscosity as a function of shear rate for slurries prepared with fines produced by ball mill grinding of primary feed under different conditions as well as by wet grinding of high-pressure roll mill product without any dispersant.

\section{THE EFFECT OF CHEMICAL ADDITIVES ON THE RHEOLOGY OF COAL-WATER SLURRIES}

The rest structure of a suspension is probably one of the most significant factors in determining its rheology [37]. The rest structure of suspensions depends on the balance of interparticle forces which can be modified by addition of suitable chemicals to the suspension [37]. There are three kinds of rest structures for concentrated suspensions: weakly flocculated, stable, and ordered stable [38]. Weakly flocculated slurries usually have very high viscosity at low shear rate, mainly because of their three-dimensional flocculated structure, and exhibit shear thinning with increased shear rates due to the breakage of such structures. Slurries with ordered rest structure exhibit a low viscosity and may undergo shear thickening at high shear rates due to an order-disorder transformation. 
The rest structure of coal-water slurries is primarily determined by the surface charge of the particles and the hydrophobic interaction between the coal particles. The formation of a water film on the coal particles, which is governed by their hydrophobicity, reduces direct particle-particle interaction during shearing, thus contributing to lower viscosity of the slurries. Increased thickness of that film, however, could reduce the amount of free water, resulting in an effective increase of the particulate volume fraction and hence the viscosity of the slurries. The formation of a sufficiently thin water film, while keeping the hydrophobic interaction between the particles to a minimum, can be achieved by suitably modifying the hydrophobicity of the coal surface. The effect of addition of vacuum oil, which is comprised of hydrocarbons having molecular weights of several hundreds, in conjunction with CoalMaster on the rheology of coal-water slurries was therefore investigated in detail.

At $67 \mathrm{wt} \%$ solids content, coal-water slurries prepared with $1 \mathrm{wt} \%$ CoalMaster as dispersant have a weakly flocculated rest structure. While this is primarily a consequence of the balance between the hydrophobic and the electrical interactions, the surface charge of the mineral matter in coal could potentially lead to flocculation through heterocoagulation. Sodium hexametaphosphate $\left[\left(\mathrm{NaPO}_{3}\right)_{6}\right]$ is a commonly used dispersant in the flotation and selective flocculation of minerals. The adsorption of charged $\left(\mathrm{PO}_{3}\right) 6^{6-}$ species on the surfaces of partially-locked as well as liberated mineral matter makes those surfaces highly negatively charged, that is similarly charged to coal particles with adsorbed CoalMaster. The effect of co-addition of sodium hexametaphosphate on the rheological behavior of coal-water slurry was also studied as part of the investigation of the effect of co-addition of reagents on the rheology of coal-water slurries.

\section{Effect of Co-addition of Sodium hexametaphosphate}

Preliminary studies showed that addition of sodium hexametaphosphate (HMP) alone as the dispersant does not improve the rheology of coal-water slurries, presumably because of its limited adsorption on coal. Experiments were therefore performed where different dosages 


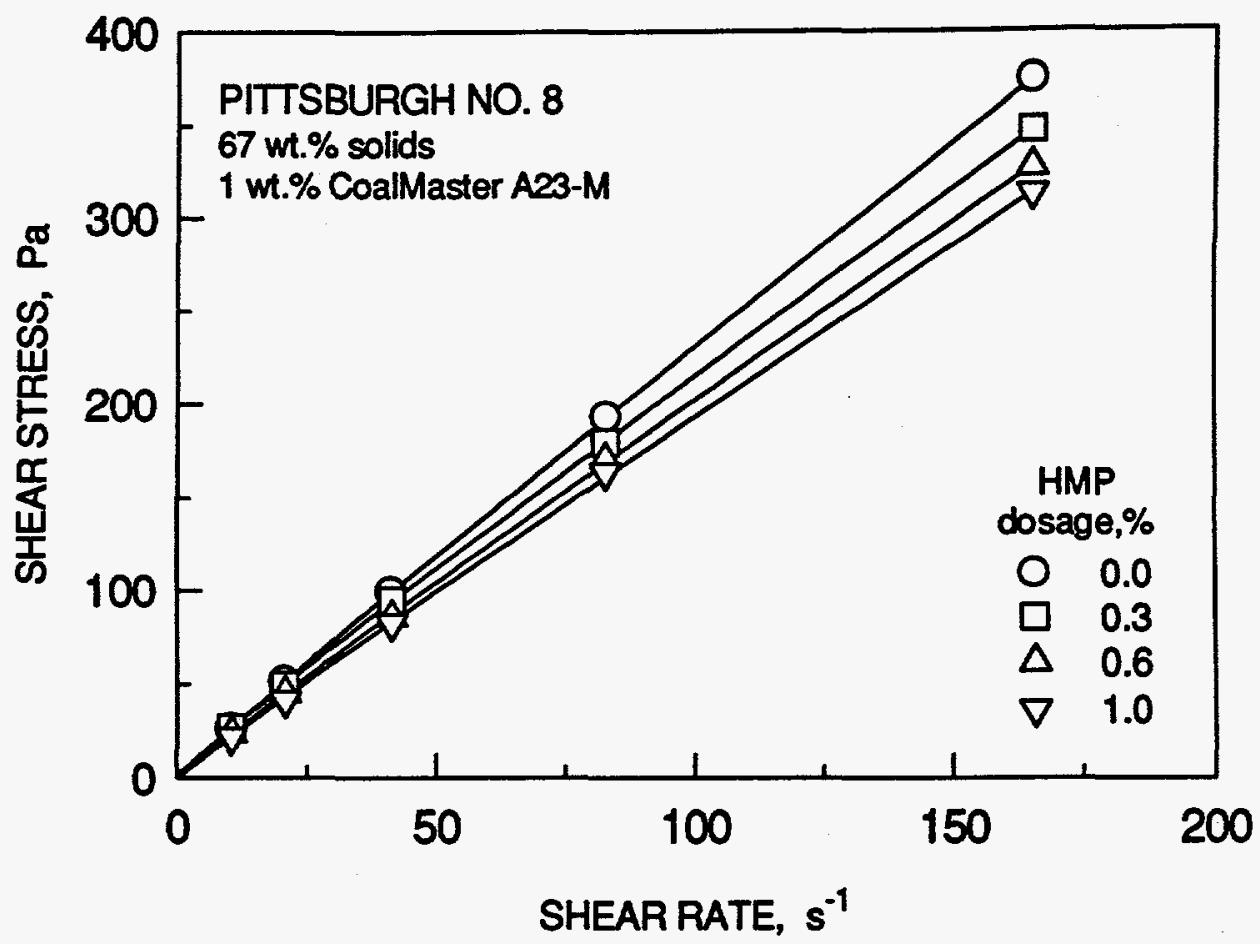

Figure 4.22. Effect of co-addition of sodium hexametaphosphate on the flow behavior of coal-water slurries.

of HMP were added to the coal-water slurry in conjunction with 1 wt. percent CoalMaster. The shear stress of these slurries as a function of the shear rate is given in Figure 4.22. While all the slurries exhibit near-Newtonian behavior, the decreasing slope of the plots with the increase in the HMP dosage indicates a progressive lowering of the viscosity of the slurries. Figure 4.23 shows the apparent viscosity at a shear rate of $100 \mathrm{~s}^{-1}$ for these slurries as a function of the HMP addition.

\section{Effect of Co-addition of Vacuum Oil}

As anticipated, the addition of vacuum oil alone significantly increased the yield stress as well as the viscosity of the slurry. This was due to increased hydrophobic attraction between the coal particles because of the oil addition. Addition of sodium hexametaphosphate, however, restored the balance between the hydrophobic and electrostatic interactions. Increasing the dosage of HMP transformed the slurry structure from a 


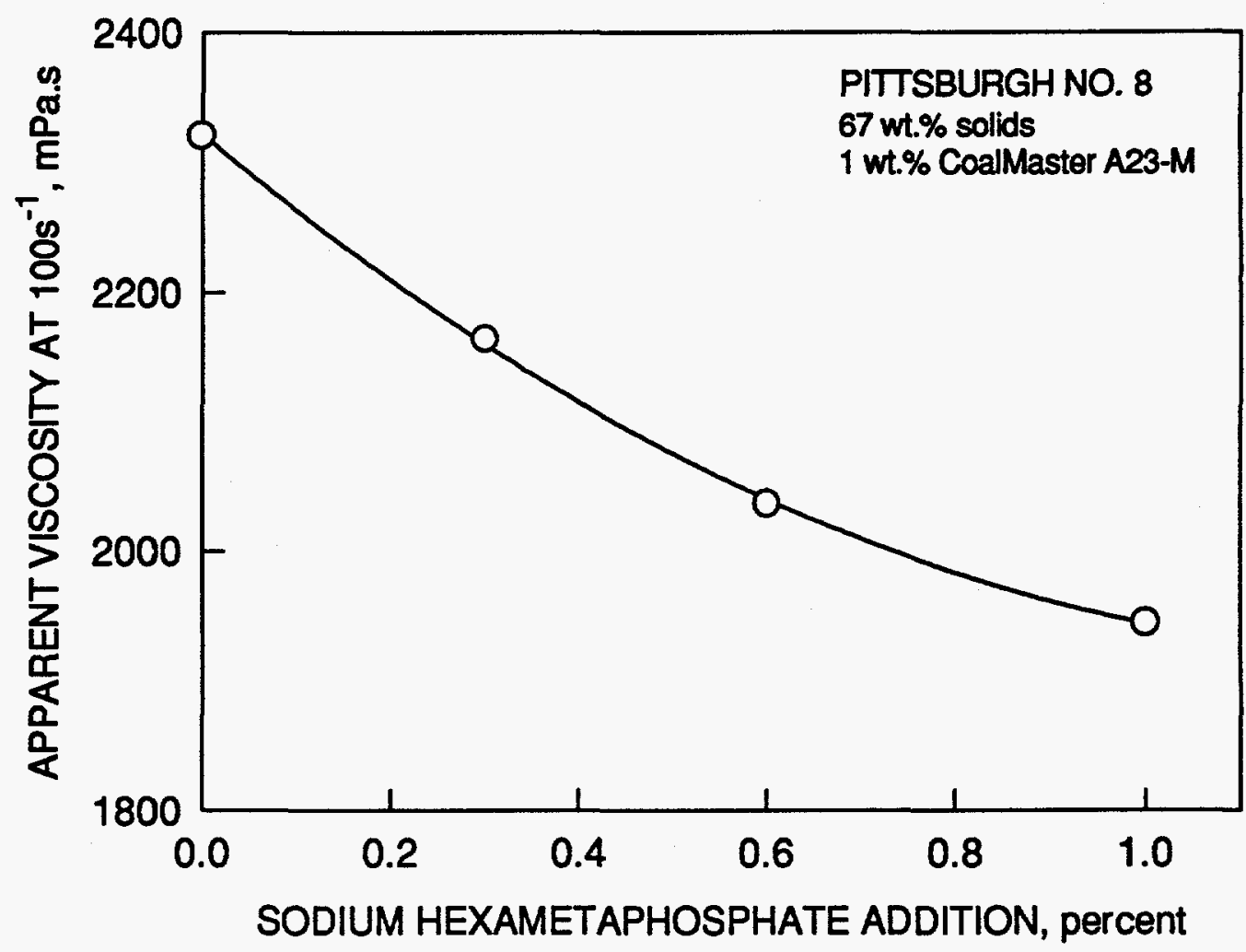

Figure 423. Effect of co-addition of sodium hexametaphosphate on the viscosity of coal-water slurries.

flocculated one to a well dispersed one. As the amount of oil is increased, the tendency to agglomerate the coal particles through coalescence of the oil film on adjacent particles may also increase. The addition of highly charged hexametaphosphate ions could prevent this coalescence through charge repulsion. The transition of the slurry structure from a flocculated state to being well dispersed one is clearly demonstrated in Figure 4.24 which shows the apparent viscosity of slurries, prepared with the addition of $1 \mathrm{wt} \%$ each of vacuum oil and CoalMaster and various dosages of HMP, as a function of the shear rate. The apparent viscosities of these slurries at shear rate of $100 \mathrm{~s}^{-1}$ are plotted in Figure 4.25 as a function of sodium hexametaphosphate dosage. Further investigations to delineate the influence of oil addition on the rheology of coal-water slurries were carried out with slurries containing 1 wt $\%$ each of CoalMaster and HMP as co-added dispersants. 


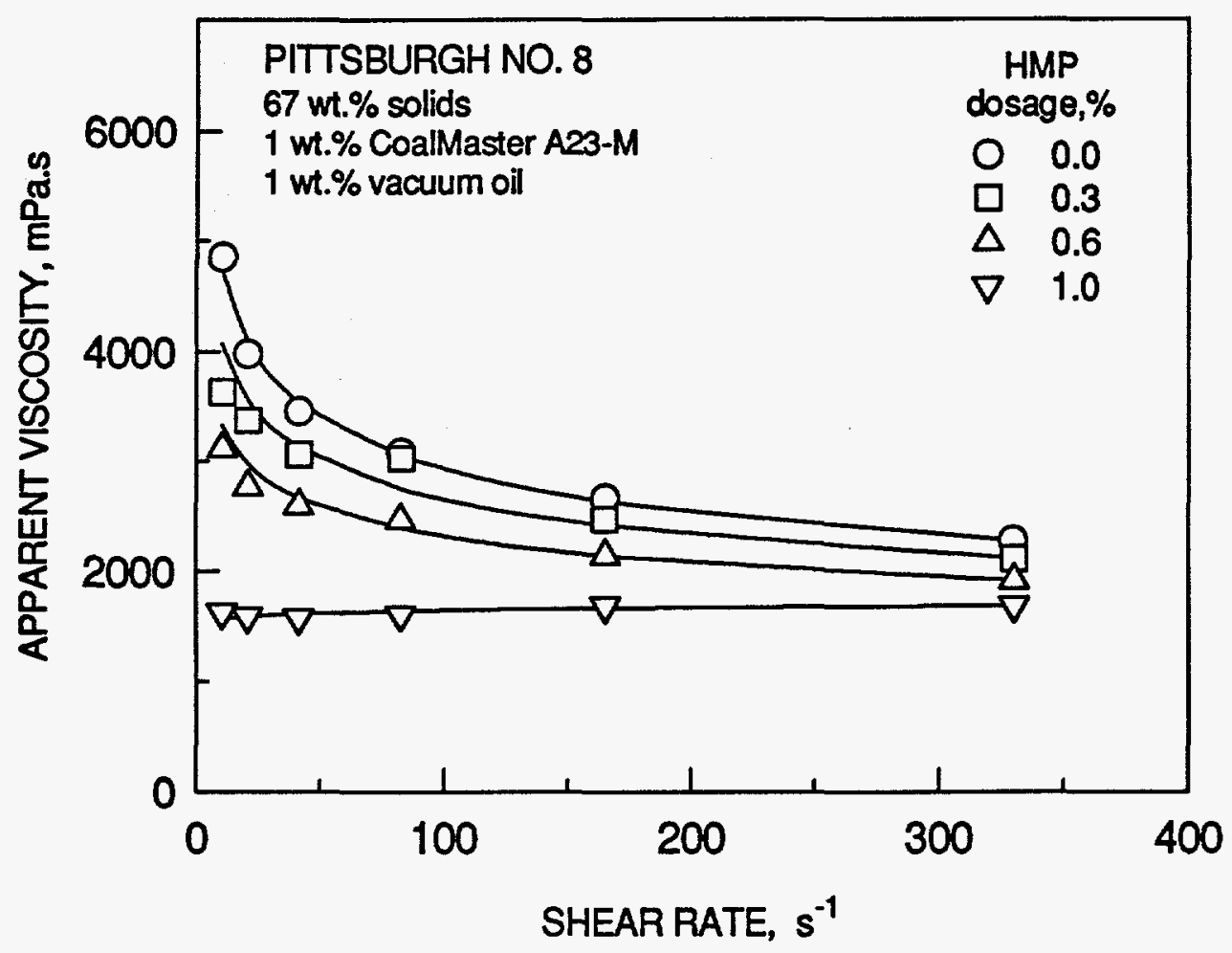

Figure 4.24. Effect of sodium hexametaphosphate addition on the structure (viscosity) of coal-water slurries prepared with CoalMaster and vacuum oil as viscosity modifiers.

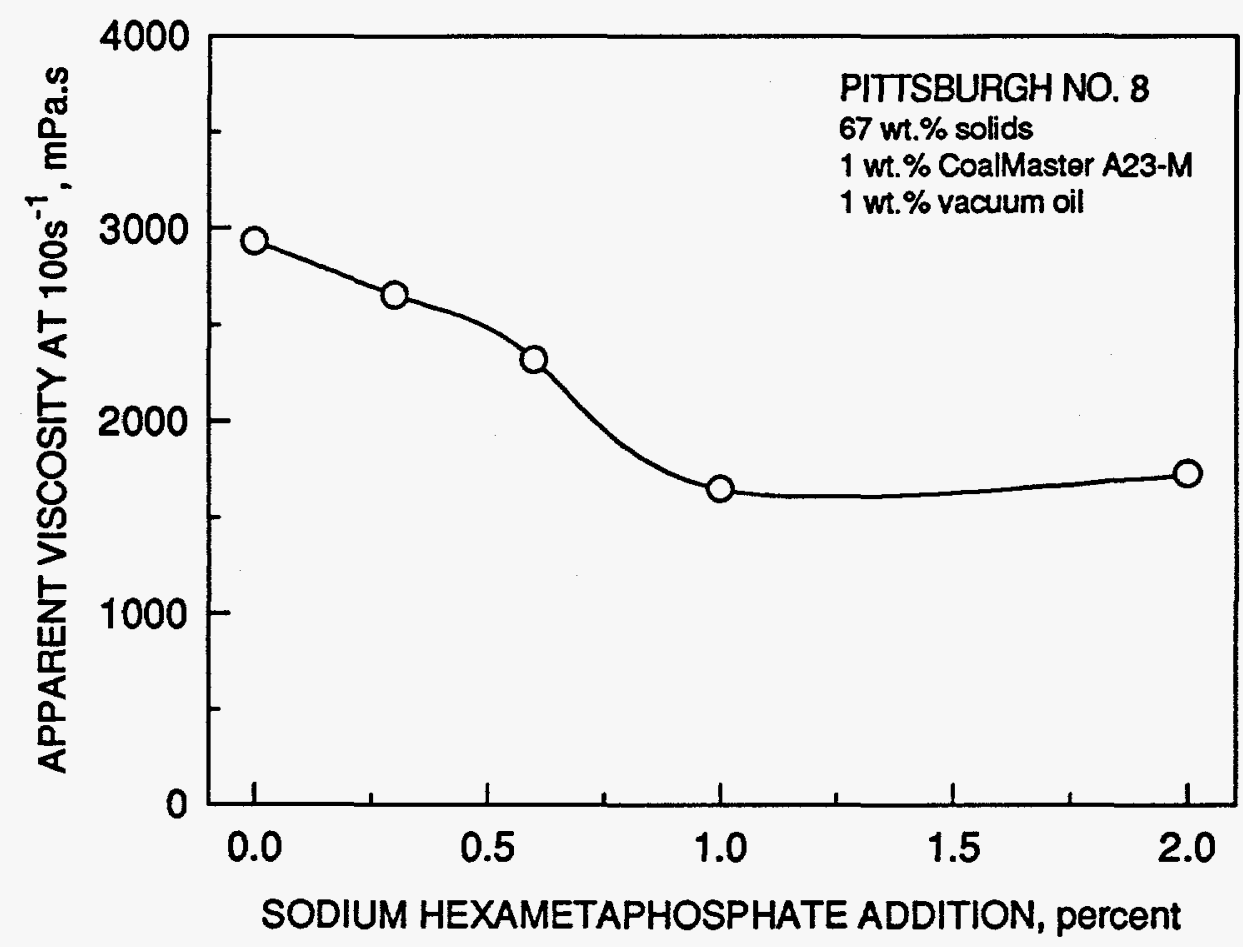

Figure 4.25. Effect of sodium hexametaphosphate dosage on the viscosity of coal-water slurries prepared with CoalMaster and vacuum oil as viscosity modifiers. 


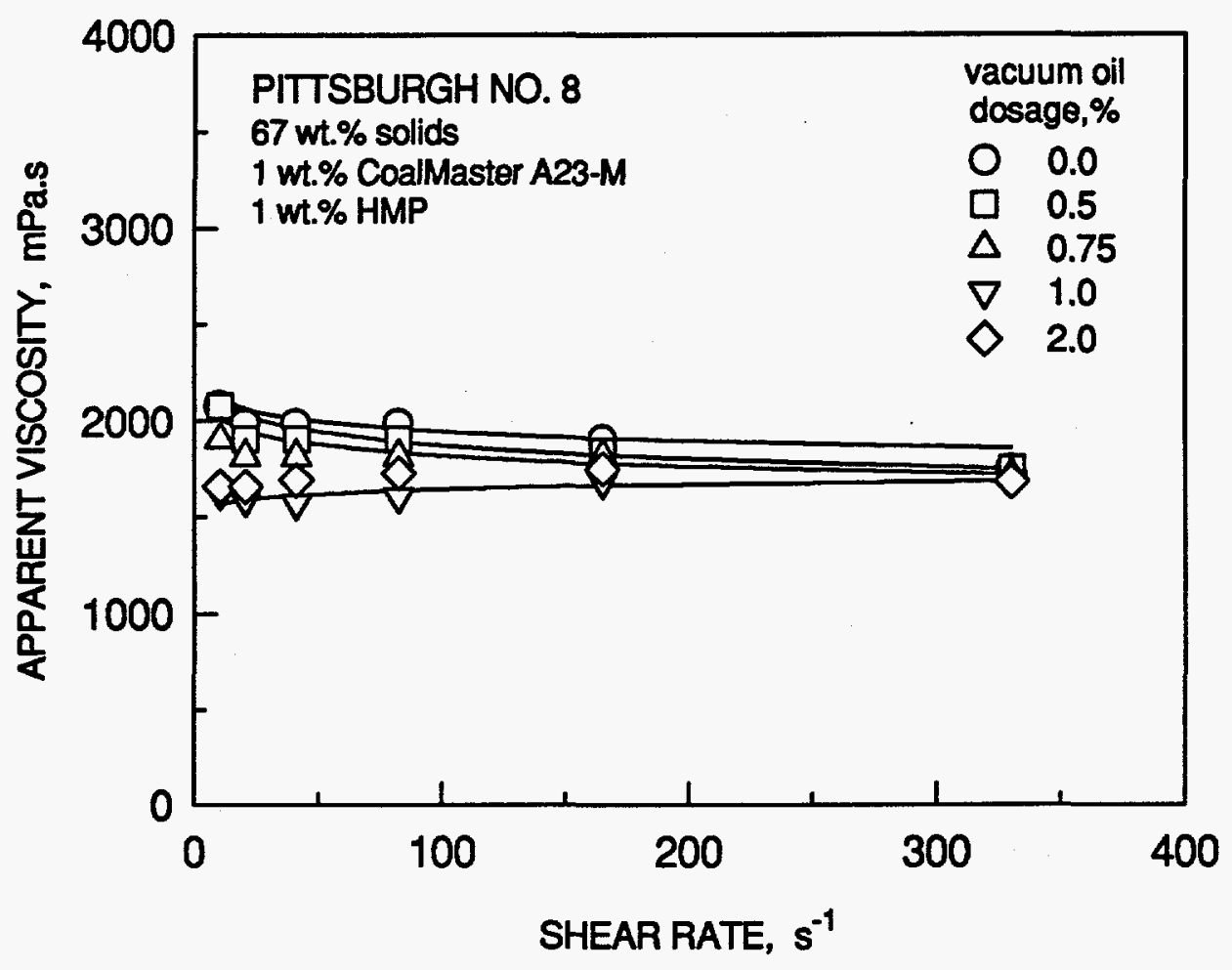

Figure 4.26. Effect of vacuum oil dosage on the viscosity of coal-water slurries prepared with CoalMaster and SHMP as dispersants.

Figure 4.26 shows the effect of oil dosage on the rheology of coal-water slurries. It is apparent from the plots in this figure that there is an optimum dosage of oil addition which results in a minimum viscosity of the slurry for a given dosage of dispersants. Unfortunately, the addition of oil does not significantly lower the viscosity.

The addition of oil lowers the interaction of water with the coal surface. The degradation of rheological properties of slurries is mainly due to the loss of free water through absorption of water in the pores of the coal particles over time and the leaching of iron from pyrite in coal by water and oxygen. Since the addition of oil reduces coal-water interaction, it was expected that oil additions should have some bearing on the aging behavior of coal-water slurries. Aging studies indicate that oil addition indeed improves the long-term aging behavior of coal-water slurries. As shown in Figure 4.27, there is no discernible change in the 


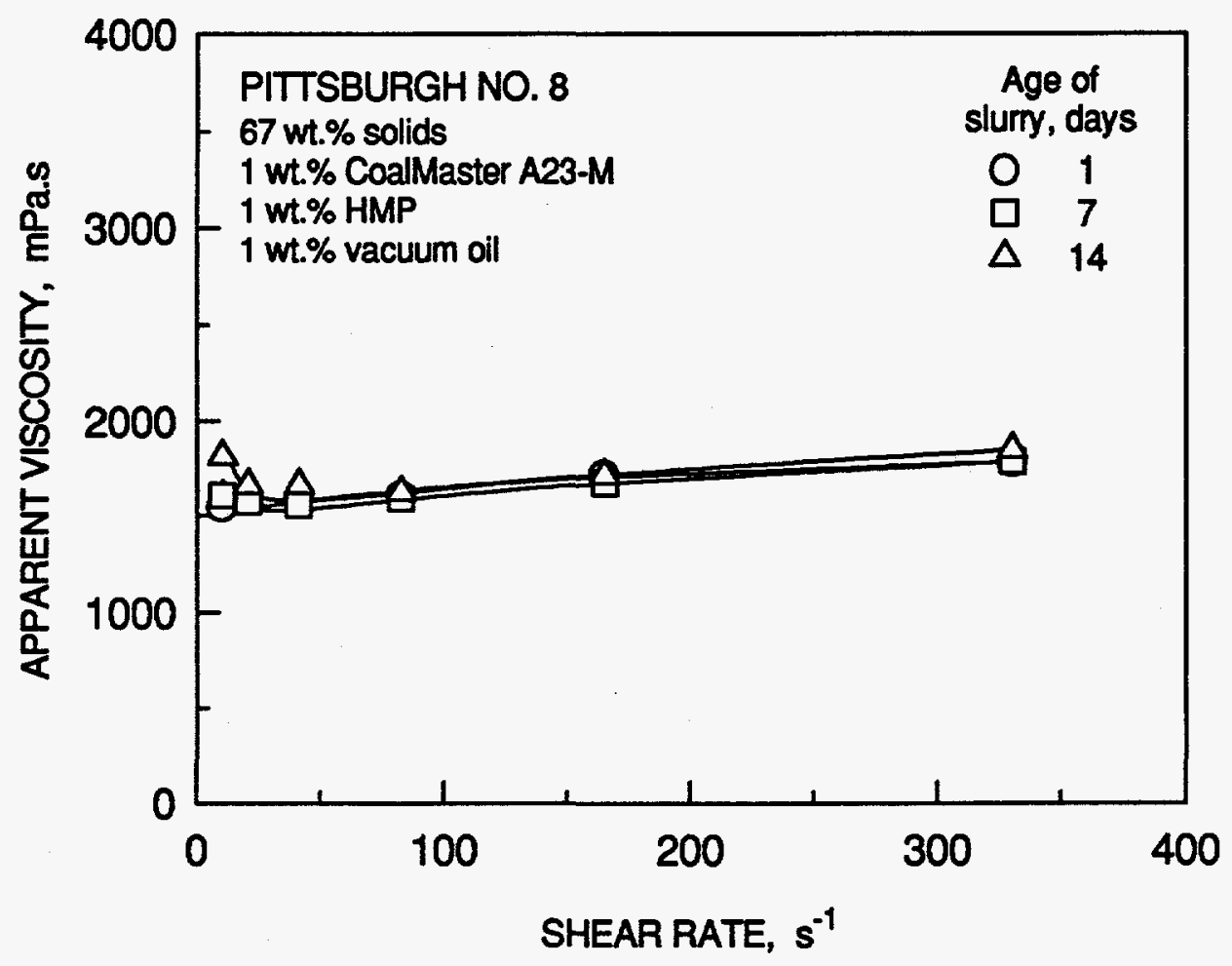

Figure 4.27. Effect of vacuum oil addition on the aging behavior of coal-water slurries.

rheological properties of slurries containing $1 \mathrm{wt} \%$ oil over a period of two weeks. The effect of co-addition of vacuum oil on the aging behavior will be discussed in detail in Section VI.

\section{Optimization of CoalMaster Dosage}

Our research has shown that co-addition of sodium hexametaphosphate and vacuum oil along with CoalMaster as viscosity modifiers improves both the short-term and long-term rheological properties of coal-water slurries. In order to minimize the reagent addition, experiments were carried out to optimize the CoalMaster addition. The results, presented in Figure 4.28, indicates that while an addition of $0.75 \mathrm{wt} \%$ CoalMaster results in the best rheological properties, CoalMaster dosage can be reduced to $0.4 \mathrm{wt} \%$ without significantly affecting the rheology of the slurries. 


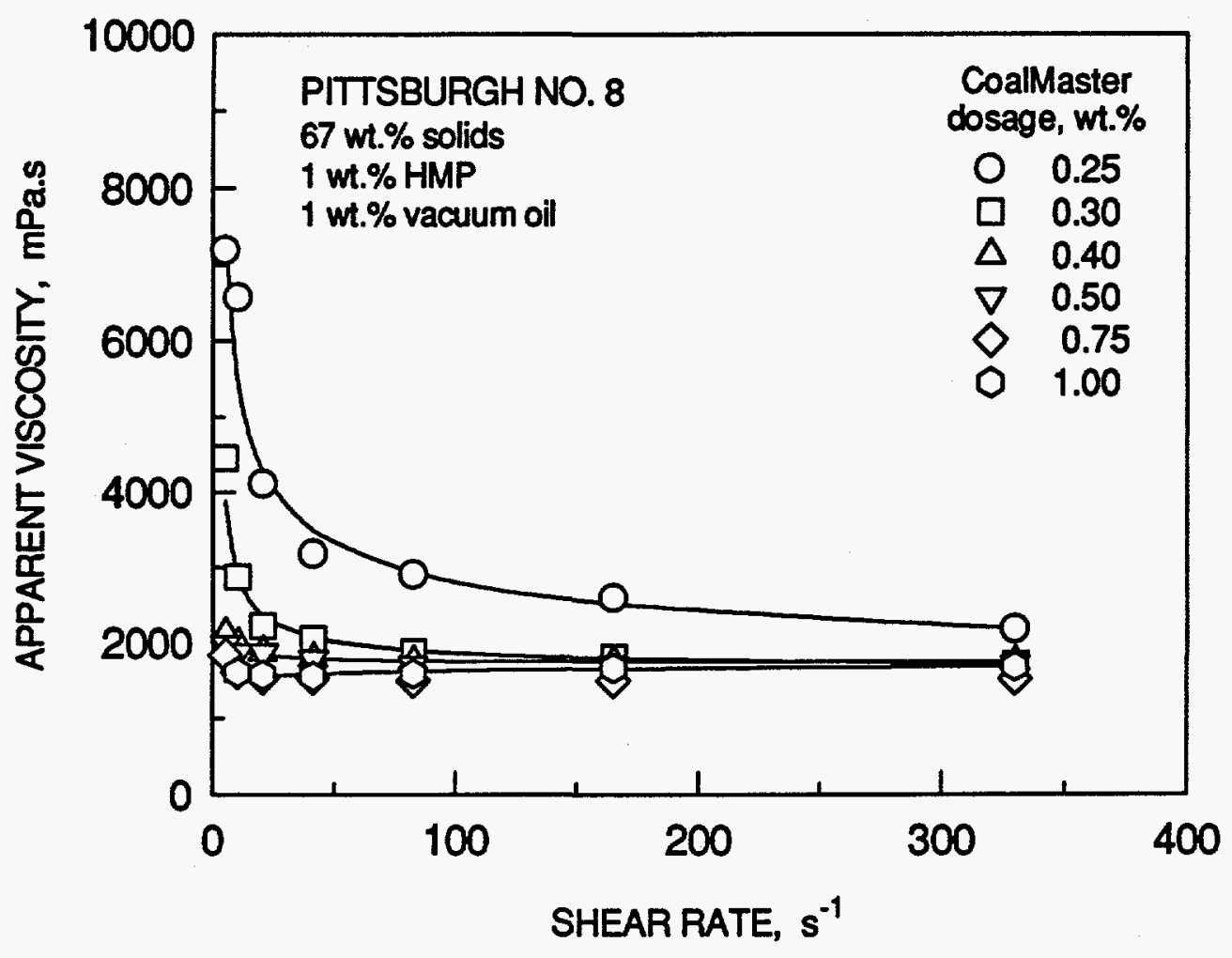

Figure 4.28. Effect of CoalMaster dosage on the viscosity of coal-water slurries prepared with vacuum oil and SHMP co-added as viscosity modifiers.

\section{SUMMARY}

The results of our studies on the influence of grinding environment on the energetics of fine grinding of coal and the rheology of coal-water slurries prepared with such fines was presented in this section. In the first part of the section, we discussed the energetics and product size distributions for fine grinding of coal in high-pressure roll mill/ball mill hybrid grinding systems. This was followed with a discussion of the relationship between the grinding environment and the rheology of coal-water slurries. Finally, we presented the results of our investigation on the effect of coaddition of reagents on the rheology of coalwater slurries prepared with fines produced by hybrid grinding of coal. 
Our research showed that significant savings in comminution energy can be achieved by grinding coal in a two-stage system, first in the high-pressure roll mill and then in a ball mill, as compared to the energy required for grinding coal to a similar degree of fineness in a single-stage ball mill system. Optimal partitioning of energy invested between the two mills is necessary, however, in order to maximize the energy savings.

Rheology mesurements showed that for slurries prepared with minus 200-mesh hybridground products, having similar median sizes, there is an inverse relationship between the viscosity of slurries and the energy expended in the high-pressure roll milling stage of the hybrid grinding experiments. We also observed that wet grinding results in slightly better rheological properties of slurries as compared to slurries prepared with fines produced by dry grinding of coal.

As reported in Section III, of the various reagents tested, CoalMaster-A23-M proved to be the most effective dispersant for the preparation of coal-water slurries having good rheological properties. In this section we showed that the rheological properties of slurries can be further improved by the co-addition of sodium hexametaphosphate and vacuum oil. Optimization studies showed that co-addition of these reagents also helps reduce the CoalMaster dosage significantly without adversely affecting the rheological behavior of slurries. 


\section{SECTION V}

\section{PACKING CHARACTERISTICS OF SOLIDS AND RHEOLOGY OF COAL-WATER SLURRIES}

In the previous sections we have looked at the effect of coal surface characteristics and their modification on the rheology of coal-water slurries. The other important factors which strongly influence the rheology of slurries are the physical characteristics of the particulate systems, namely, particle shape and size distribution (which controls the particle packing). The viscosity of slurries with high solids content is strongly influenced by the packing density of the feed material. The packing density can also be significantly altered by mixing distributions of different median sizes and, to some extent, by modifying the grinding environment. We have shown in Section IV, that the mode of comminution, which can alter the particle shape and size distribution to some extent, has a definite influence on the rheology of coal-water slurries. This section, summarizes the results of our research directed towards: i) establishing the relationship between the packing characteristic of fines and the viscosity of slurries prepared with the fines, and ii) investigation of the effect of mixing distributions on the rheology.

\section{RELATIONSHIP BETWEEN THE PACKING DENSITY OF FEED AND THE VISCOSITY OF SLURRY}

The viscosity of concentrated suspensions of non-interacting particles is primarily determined by the resistance to the flow of liquid through the interparticle space. Consequently, rheology of coal-water slurries at high solids content is strongly influenced by the packing characteristics of the particles, provided that the particulate surface characteristics are similar. Improvement in the rheology of dense slurries can be achieved by modifying the packing density of the solids. In this section, we will attempt to correlate the rheological behavior of slurries with the packing density of the feed material. 


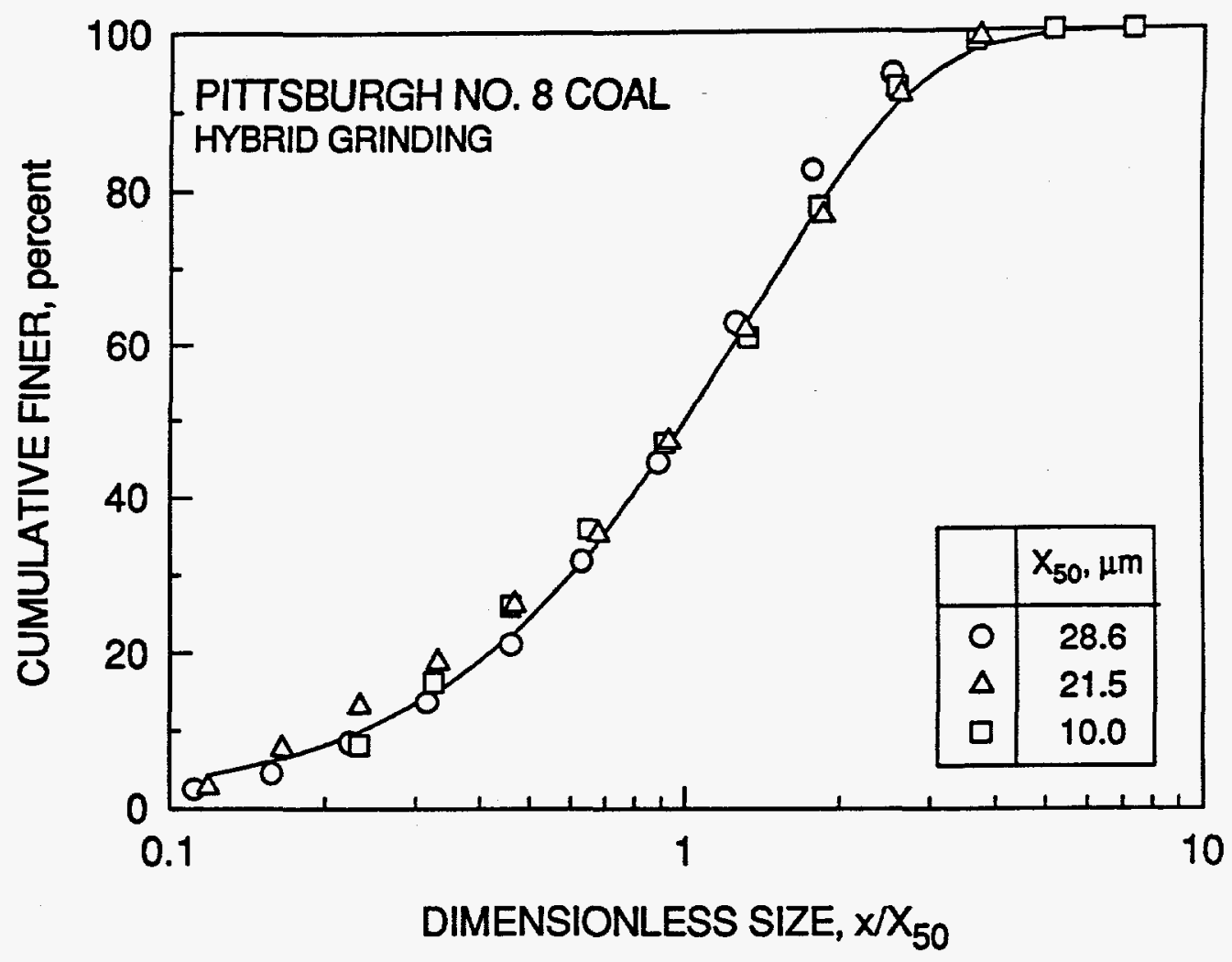

Figure 5.1. Self-similarity of product size distributions, generated by hybrid grinding of coal.

First, a minus 8-mesh coal sample was ground in the high-pressure roll mill and then reground in a 10-inch ball mill with respective energy expenditures of $2.0 \mathrm{kWh} / \mathrm{t}$ and $1.0 \mathrm{kWh} / \mathrm{t}$. The ball mill discharge was screened at 150 mesh and the undersize (median size being $28 \mu \mathrm{m}$ ) was further ground in a 5 -inch ball mill to median sizes of 22 and 10 microns. The size distributions are self-similar, as shown in Figure 5.1 where the cumulative finer distribution is plotted against particle size normalized with respect to the median size of the distribution. The distributions could be mathematically represented by the following equation:

$$
F\left(x / X_{50}\right)=1-\exp \left[-\ln 2\left(x / X_{50}\right)^{n}\right]
$$

The packing densities of the fine products were measured using a tap density machine. The fines were used to prepare coal-water slurries containing $61,63,65,66$ and 67 weight percent solids. Apparent viscosities of these slurries were determined and the results are 


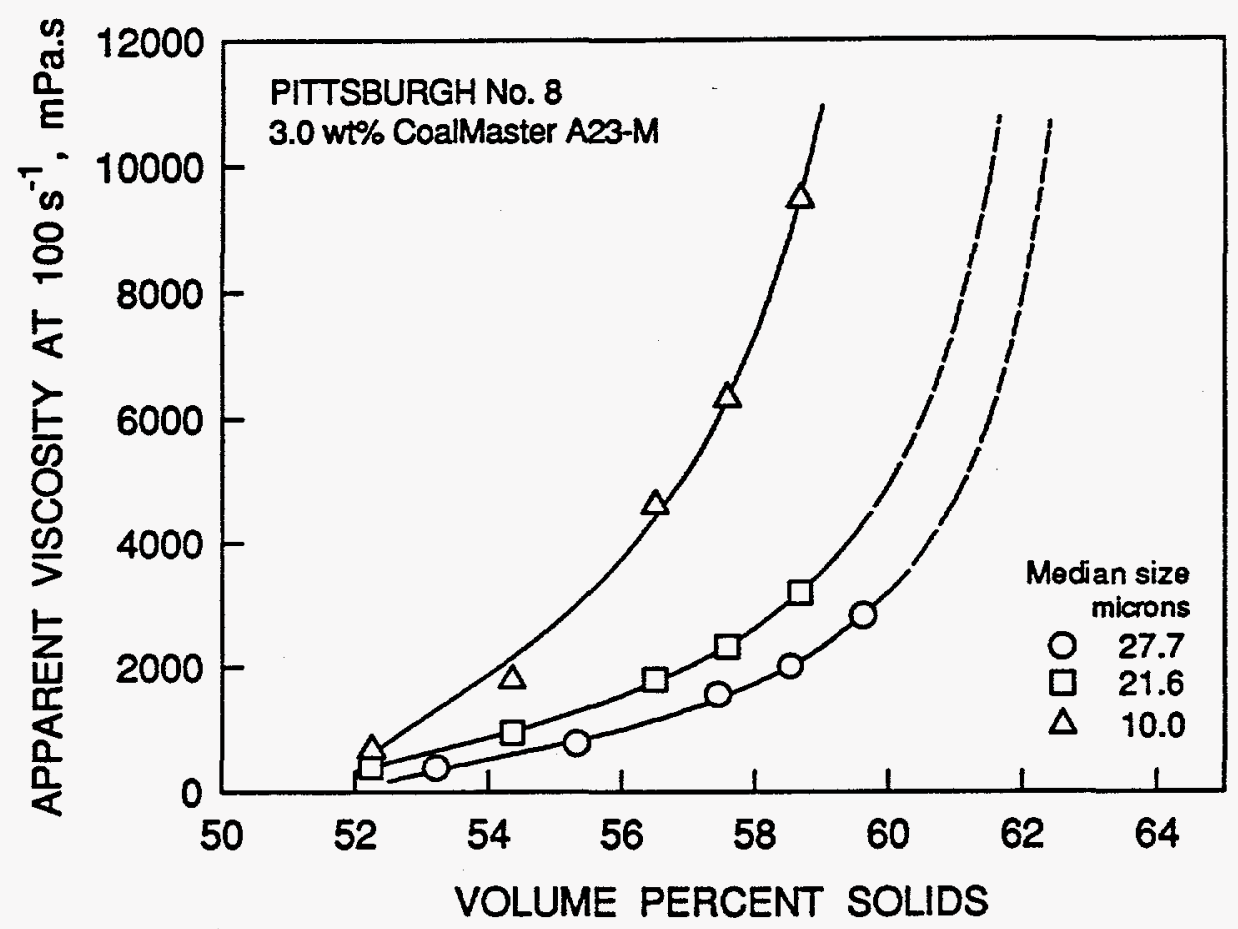

Figure 5.2. Apparent viscosity of coal-water slurries, prepared with fine feed of various median sizes, as a function of volume percent solids in the slurry.

plotted as a function of solids content (by volume) in Figure 5.2. As can be seen from Figure 5.2, there is a nonlinear increase in the apparent viscosity, at any given solids content, with increased fineness of the feed. The estimated maximum volume percent solids are plotted in Figure 5.3 against the tap densities of the feeds. Figure 5.3 suggests that there is a linear dependence between the tap density and the maximum volume percent solids. It turns out that for unimodal, self-similar distributions, the tap density increases exponentially with the increase in the median size of the distribution, as shown in Figure 5.4.

\section{EFFECT OF MIXING FEED DISTRIBUTIONS ON THE PACKING DENSITY OF COAL FEED AND THE RHEOLOGY OF COAL-WATER SLURRIES}

In the previous sub-section, we established the relationship between the packing density of the ground coal and the maximum possible solids content of coal-water slurries prepared with those ground products. We observed that an increase in the packing density of the feed results in a linear increase in the maximum possible solids content of the slurry. This 


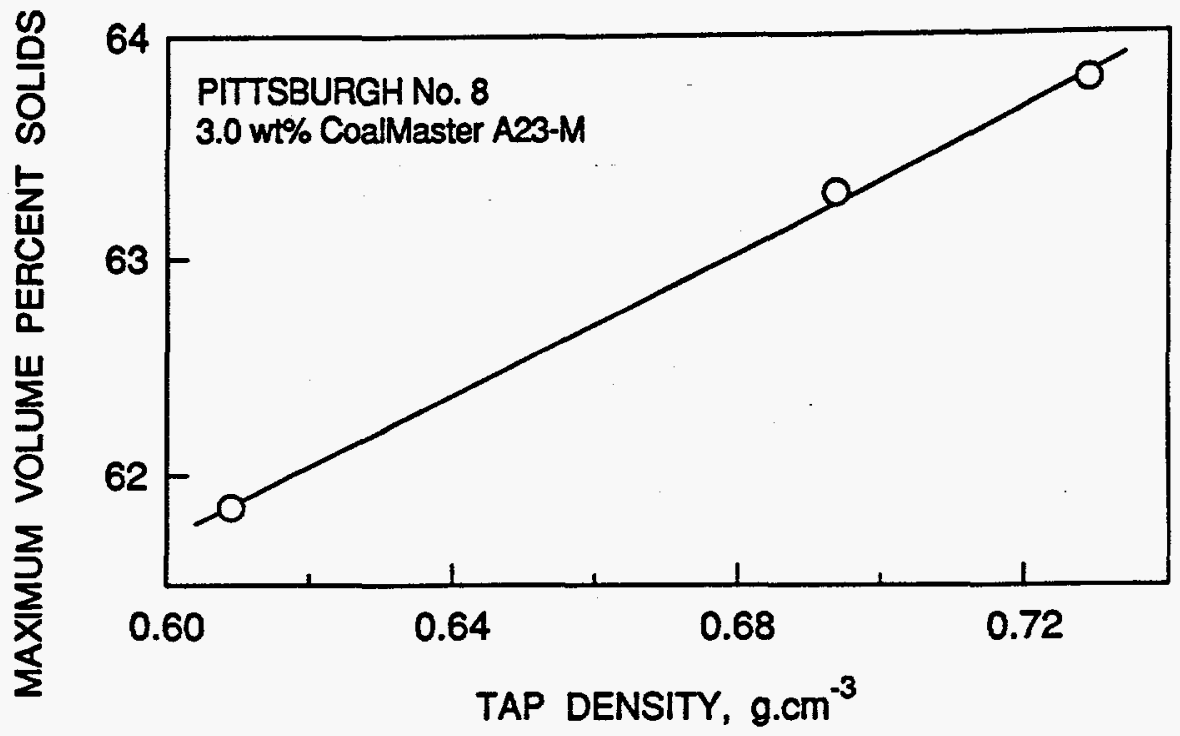

Figure 5.3. Relationship between the tap-density of the feed and the maximum volume percent solids in the slurry.

implies that any improvement in the packing density of the feed material should result in a corresponding decrease in the viscosity of a slurry, for a given solids content, prepared with such feed.

It is well-known that mixing different particle sizes increases the packing density, especially when the particles are very different in size. The improved packing density is a consequence of the smaller particles filling the interstices between the packed coarse particles.

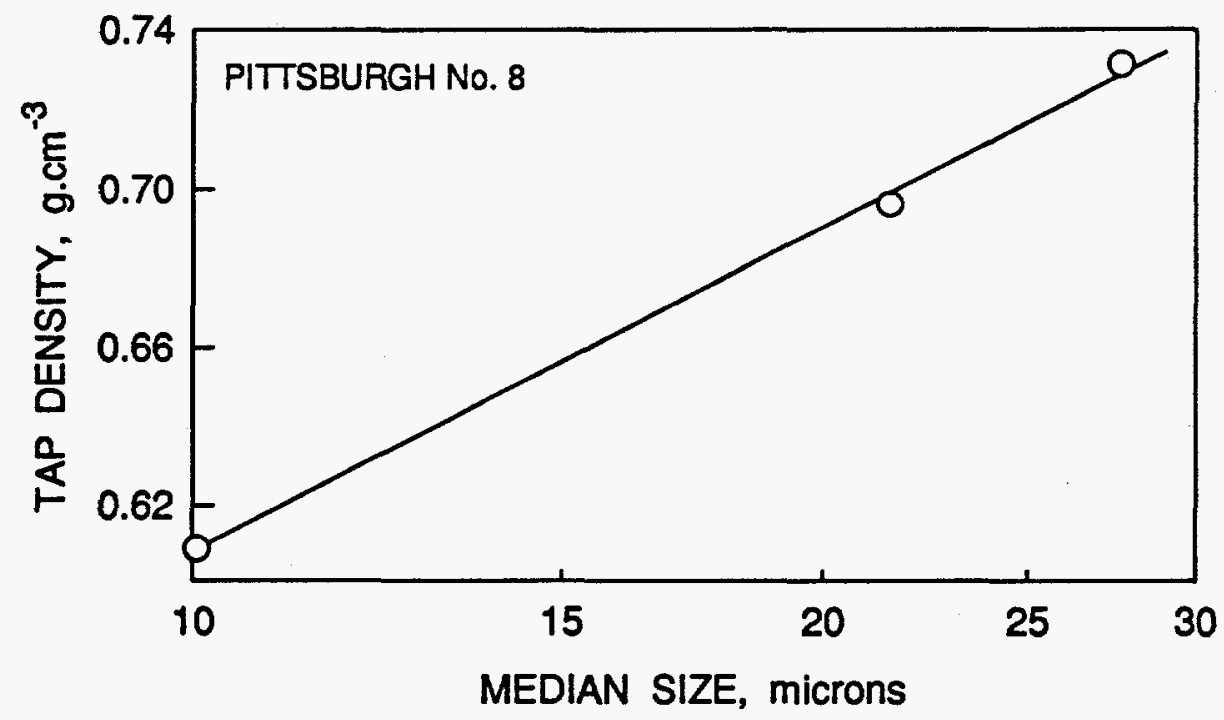

Figure 5.4. Relationship between the median size and the tap-density of fine coal products. 
For every ratio of particle sizes, there is a composition that will optimize the packing density. Continuous particle size distributions can also be mixed to improve the packing density. A density benefit from such a mixture requires a large difference in the mean sizes of the two distributions. As in the case of mixing different particle sizes, the packing density of the mixed distributions increases as the ratio of the mean particle sizes increases. The results of our studies on the effect of mixing distributions on the packing density of fine coal feed and the viscosity of coal-water slurries prepared with such ground products are presented here.

The three ground coals were used for these studies and are designated as coarse (C), medium (M), and fine (F) distributions, their median sizes being 28,22 and $10 \mu \mathrm{m}$, respectively. Four binary mixtures were investigated - three of these were mixtures of medium and fine distributions with $\mathrm{M} / \mathrm{F}$ ratios of $4: 1,2: 1$, and $1: 1$, and the fourth was a coarse-fine mixture with $\mathrm{C} / \mathrm{F}$ ratio of 7:3. Coal-water slurries, with $61,63,65,66$ and 67 weight percent solids content, were prepared using each of the feed mixtures.

The tap densities of the mixtures as well as the original distributions are plotted in Figure 5.5 against the logarithm of median size of the feeds. As is apparent from the figure, mixed distributions have higher packing densities than those of a single distribution at the same median size. The improvement is more pronounced in the case of coarse-fine mixture.

Figure 5.6 gives the apparent viscosities of the coal-water slurries of various solids contents as a function of the fraction of the medium distribution in the M-F mixtures. As can be seen from this figure, except at the highest solids content where the viscosity increases almost exponentially with increasing fraction of fines, the addition of the fine-component up to about 30 percent either lowers the viscosity or leaves it unchanged. The apparent viscosities of slurries prepared with the mixed distributions are plotted as a function of the median size of the distributions in Figure 5.7. The apparent viscosities of single distributions are also shown in the figure for the sake of comparison. The benefit of mixing distributions is even more clear from this figure when one compares the plots for the single distributions with those for the mixed distributions. The results presented in the figure also show the superiority of the 


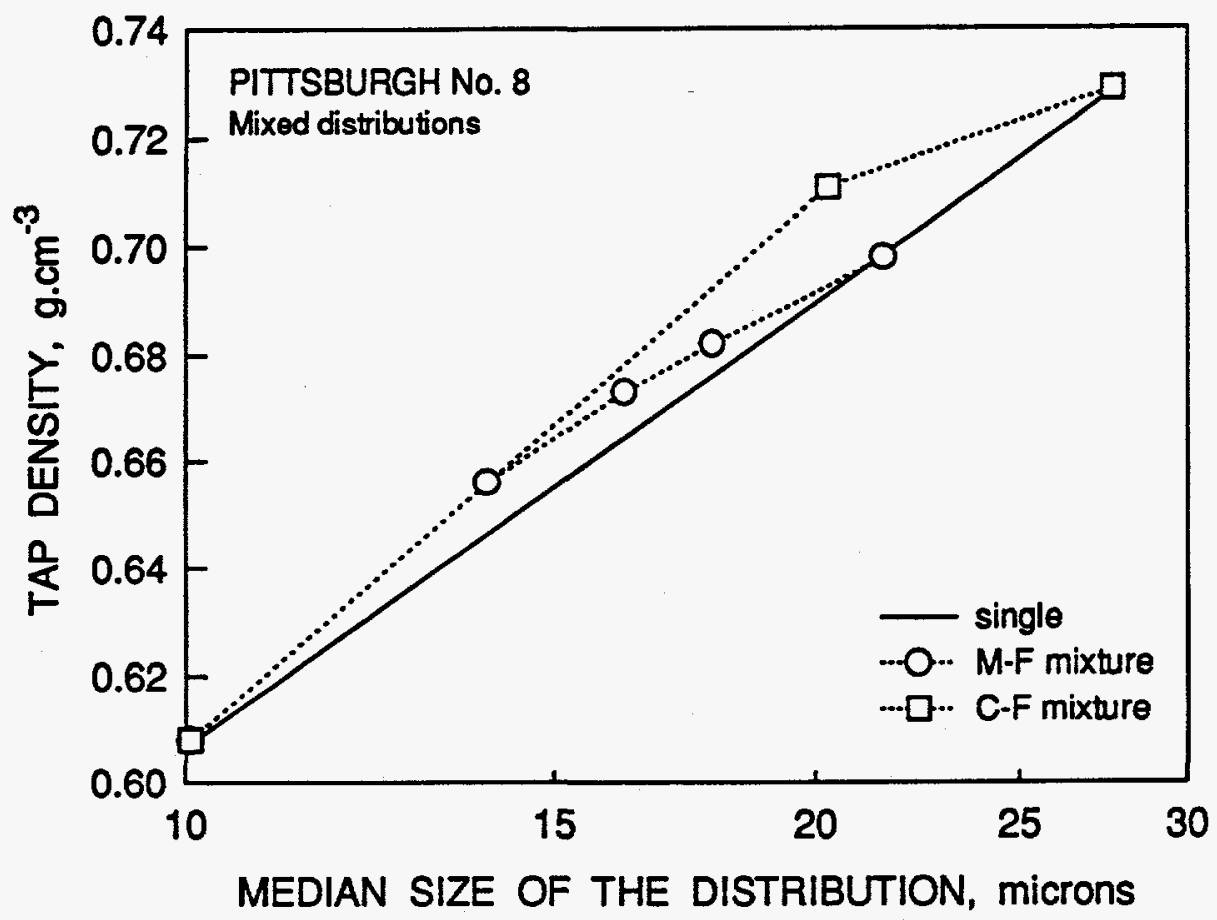

Figure 5.5. The effect of mixing distributions on the median size and the packing density.

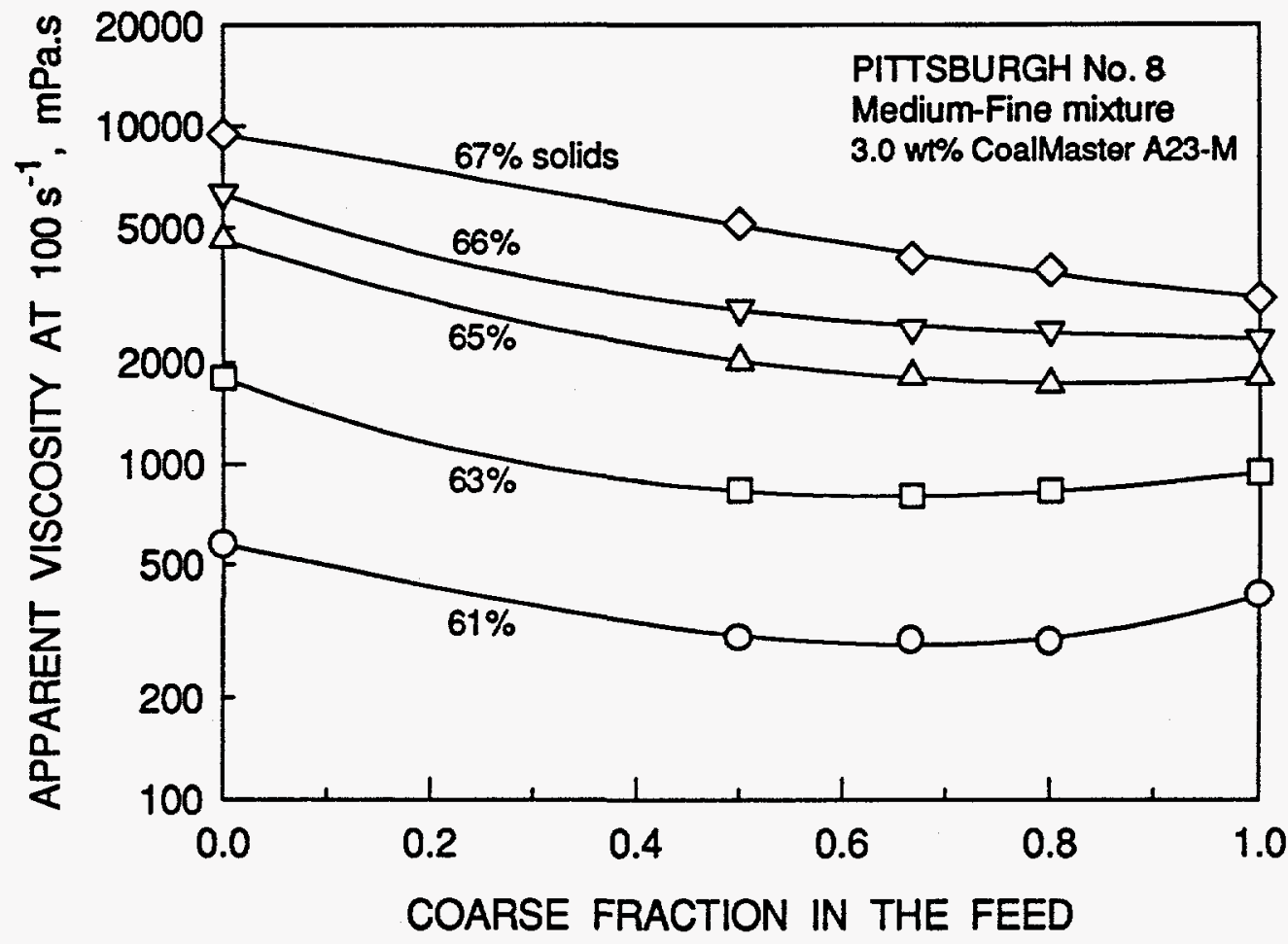

Figure 5.6. The effect of the fraction of coarse component(M) in medium-fine mixed distributions on the apparent viscosity of slurries prepared with such mixed feeds. 


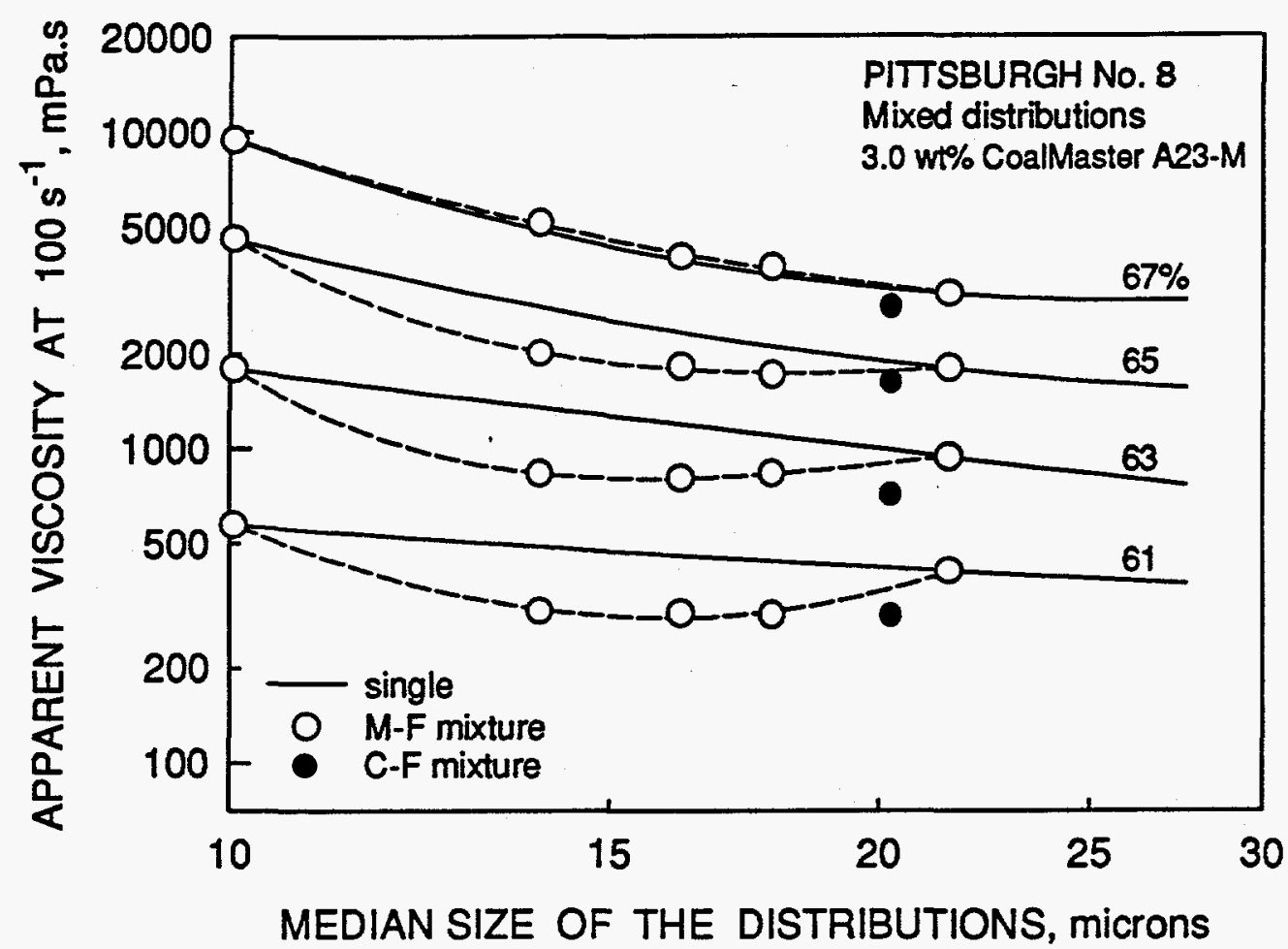

Figure 5.7. Comparison of the apparent viscosity of slurries prepared with single-component feeds with that of slurries prepared with mixed feeds.

C-F mixture over M-F mixtures. This is consistent with the observation made earlier regarding the effect of the ratio of the median sizes of the distributions on the packing density.

Let us define a relative viscosity as the ratio of viscosity of a slurry prepared with feed having a mixed distribution to that of a slurry prepared with one-component distributed feed, having the same median size as the mixed distribution. An empirical correlation between the viscosity, $\mu$, of a suspension and the volume fraction of solids in the suspension is given by [39]:

$$
\mu=\mu_{0}\left[1+\mathrm{R} \frac{\phi / \phi_{\mathrm{m}}}{1-\phi / \phi_{\mathrm{m}}}\right]^{2}
$$

where $\mu_{\mathrm{o}}$ is the viscosity of the suspending medium, $\phi$ is the volume fraction of solids, and $\phi_{\mathrm{m}}$ is the maximum achievable volume fraction of solids. $R$ is a scalar, taken as 0.75 by the authors. If we assume $\mathrm{R}$ to be unity, we obtain the following expression for the relative 
viscosity, $\mu_{\mathrm{r}}$ :

$$
\mu_{\mathrm{r}}=\left[\frac{1}{1+\frac{\phi_{\mathrm{m}, \mathrm{x}}-\phi_{\mathrm{m}, \mathrm{s}}}{\phi_{\mathrm{m}, \mathrm{s}}-\phi}} \cdot \frac{\phi_{\mathrm{m}, \mathrm{x}}}{\phi_{\mathrm{m}, \mathrm{s}}}\right]^{2}
$$

where $\phi$ is the volume fraction of solids, $\phi_{m, s}$ is the maximum achievable volume fraction of solids for a single-component system, and $\phi_{m, x}$ is the maximum achievable volume fraction of solids for a mixed-component system for a given fraction of coarse component.

Since a linear relationship exists between the tap density and the maximum achievable volume fraction of solids (Figure 5.3), Equation 5.3 could also be written in terms of the tap densities. In addition, the difference in the tap densities of a mixed distribution and a singlecomponent distribution having the same median size is a function of the fraction of coarse component, $\mathrm{x}_{\mathrm{c}}$. Equation 5.3 could then be rewritten as:

$$
\mu_{\mathrm{r}}=\left[\left(1+\frac{\mathrm{f}\left(\mathrm{x}_{\mathrm{c}}\right)}{\phi_{\mathrm{m}, \mathrm{s}}-\phi}\right) \cdot \frac{\rho_{\mathrm{m}, \mathrm{s}}}{\rho_{\mathrm{m}, \mathrm{x}}}\right]^{-2}
$$

where $\rho_{m, s}$ is the tap density of the single-component system, $\rho_{m, x}$ is the tap density of the mixed-component system, and $f\left(x_{c}\right)$ denotes the functional relationship between the difference in the tap densities and the coarse fraction in the mixture.

As suggested by Equation 5.4, plots of the relative viscosities as function of the coarse fraction in the feed, shown in Figure 5.8, indeed exhibit a parabolic relationship. However, the trend with respect to the solids content is opposite to that indicated by Equation 5.4. This is probably because of increased particle-particle interaction at higher solids content, under Couette flow conditions, and hence a deviation from the ideal packing.

As illustrated in Table 5.1, Equation 5.1 can be used to accurately calculate the median size of the mixed distributions. A simple measurement of the tap densities of the mixed distributions, therefore, would allow us to predict the relative viscosities of mixed 


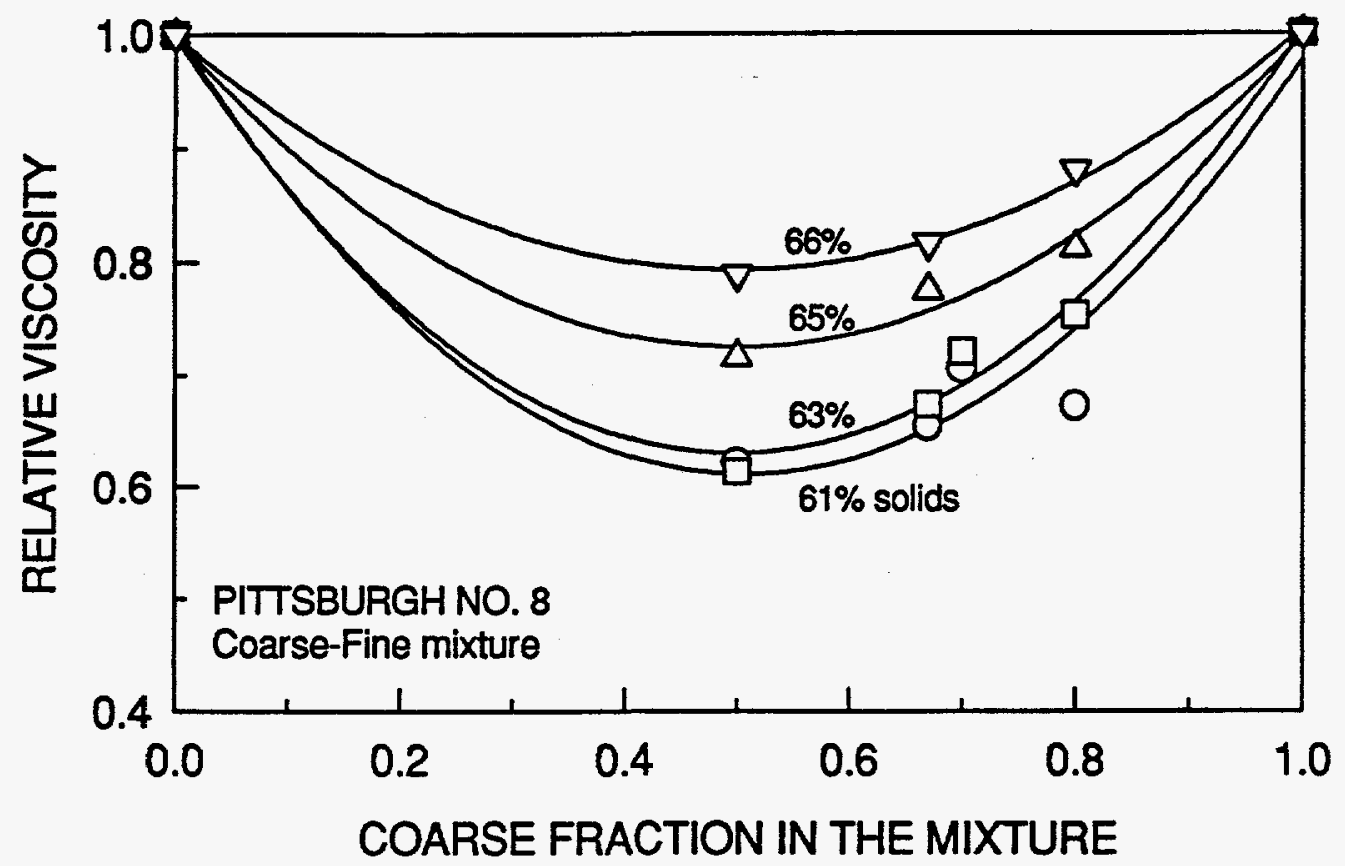

Figure 5.8. Relative viscosity as a function of the fraction of coarse component in the feed comprising of mixed distributions.

distributions as a function of the coarse component in the mixture with a fair degree of accuracy.

Table 5.1. Measured and computed median sizes for mixed distributions.

\begin{tabular}{|ccc|ccc|ccc|}
\hline \multicolumn{3}{|c|}{ Mixture 1 } & \multicolumn{3}{c|}{ Mixture 2 } & \multicolumn{3}{c|}{ Mixture 3 } \\
\hline C:F & $\mathrm{X}_{50}$, exp. & $\mathrm{X}_{50}$, calc. & C:F & $\mathrm{X}_{50}$, exp. & $\mathrm{X}_{50}$, calc. & C:F & $\mathrm{X}_{50}$, exp. & $\mathrm{X}_{50}$, calc. \\
\hline $1: 0$ & 22.71 & 22.71 & $1: 0$ & 21.55 & 21.55 & $1: 0$ & 27.68 & 27.68 \\
$4: 1$ & 19.26 & 19.75 & $4: 1$ & 17.85 & 18.03 & $7: 3$ & 20.26 & 20.20 \\
$2: 1$ & 18.01 & 18.17 & $2: 1$ & 16.20 & 16.32 & $0: 1$ & 10.05 & 10.05 \\
$1: 1$ & 16.09 & 16.34 & $1: 1$ & 13.93 & 14.10 & & & \\
$1: 2$ & 14.67 & 14.80 & $0: 1$ & 10.05 & 10.05 & & & \\
$1: 4$ & 13.79 & 13.87 & & & & & & \\
$0: 1$ & 12.46 & 12.46 & & & & & & \\
\hline
\end{tabular}




\section{SUMMARY}

For unimodal distribution of particle size, except for a slight deviation at very high solids content, where there is significant particle-particle interaction, there exists a power law relationship between the viscosity of coal-water slurries and the median size of the solids. The size distributions, however, are self-similar irrespective of the fineness of grind. This selfsimilarity allows to accurately compute the median size of mixed distributions comprising of coarse and fine fractions. There is also a direct correspondence between the dry packing density, as determined by tap density measurements, and the maximum achievable solids content of the slurries.

Bimodal distributions, generated by mixing coarse and fine distributions, have higher packing densities compared to those of a unimodal distribution with similar median size. The viscosities of slurries, prepared with a bimodally-distributed feed, are also significantly lower than those of slurries prepared with feed having unimodal distribution of identical median size.

Experimental data, as well as theoretical analysis, indicate a parabolic relationship between the relative viscosity, defined as the ratio of viscosity of a slurry prepared with feed having a bimodal distribution to that of a slurry prepared with feed having a undimodal particle size distribution and the same median size as the bimodal distribution, and the fraction of coarse component in the bimodal distribution. The foregoing correlations should allow a fairly accurate computation of the relative viscosities of slurries prepared with feeds having bimodal distributions through measurement of the tap densities of mixed distributions. 


\section{SECTION VI}

\section{AGING BEHAVIOR OF SLURRIES}

Freshly prepared coal-water slurries may need to be stored for extended periods of time prior to their burning as a fuel. Transport of slurries through pipelines over long distances would also result in significant aging of the slurries before they are used. Oxidation of coal over time will change the surface characteristics of coal particles. It is therefore essential to study the effect of aging of coal-water slurries on their rheological behavior. Water-soluble inorganic and organic matter in coal as well as the oxidation products will be leached out over time and may adversely affect the long-term behavior of the slurries. The effect of slurry aging on the rheology of coal-water slurries were therefore investigated in detail. Research was carried out to delineate the principal causes for the degradation of slurries over time and minimize the adverse effects of aging on slurry properties.

Several phenomena can occur during the aging of coal-water slurries and contribute to the degradation of their rheological properties:

i) Imbibition of water in the micropores in coal over time could reduce the effective volume of the aqueous phase, in other words, increase the volume fraction of solids.

This results in a nonlinear increase in slurry viscosity over time, similar to that caused by increase in solids content of the slurries.

ii) Oxidation the of coal surface would decrease its hydrophobicity which, in turn, would increase the wetting of coal particles by water and the thickness of stable water film around the particles. Increased wetting of particles may lower the viscosity up to a point by reducing particle-particle friction, but this is counteracted by the increase in the immobilized layer of water on the particle surface (reduction in the free water) that leads to an increase in the viscosity.

iii) Oxidation of pyrite in coal releases iron, which may adsorb on the negatively charged coal surface, thereby lowering the surface charge on the particles. Adsorption of 
positively charged ferrous and ferric ions on coal particles may also lead to heterocoagulation. Both of these phenomena can significantly affect the structure and stability of slurries. Furthermore, the hydration of these ions will also lower the free water content of the slurry.

Our preliminary studies indicated that a significant amount of iron is present in the aqueous phase of slurries prepared with coal that had been stored in air for an extended time period. Sadler and Bethany [40] had investigated how different cations affected the viscosity of lignite-water slurries, and showed that multivalent cations are the major "culprit" in increasing slurry viscosity. Kaji and coworkers [41] studied the effects of counterions on the viscosity of coal-water slurries and found that anions do not influence the viscosity significantly but that such multivalent hydrolizable cations as $\mathrm{Al}^{3+}$ or $\mathrm{Ce}^{3+}$ increase the slurry viscosity enormously after a certain concentration is reached. Unfortunately, they did not investigate the effect of iron on slurry viscosity. The focus of our research on the aging behavior of coal-water slurries was, therefore, primarily on the investigation of the influence of dissolved iron on rheology.

\section{INFLUENCE OF IRON ON THE RHEOLOGY OF COAL-WATER SLURRIES}

Freshly prepared coal-water slurries, made with fines that had been stored for an extended period of time, contained significant amounts of iron in the aqueous phase $\left(3 \times 10^{-4} \mathrm{M}\right)$. The slurries exhibited a continuous increase in viscosity over time, as shown in Figure 6.1. Besides the increase in viscosity, the rheological behavior changes from nearNewtonian for a freshly preapred slurry to pseudoplastic with significant yield stress after the slurry has aged for about two weeks, as indicated by the flow curves and the viscosity plots shown in Figure 6.2. The concentration of CoalMaster in the aqueous phase of these slurries was also monitored over time. For determining the concentration of CoalMaster in the slurry liquid, a one-gram sample of the slurry was withdrawn and first suspended in $19 \mathrm{ml}$ of distilled water. The suspension was centrifuged at $12,000 \mathrm{rpm}$ for a half hour and the concentration of 


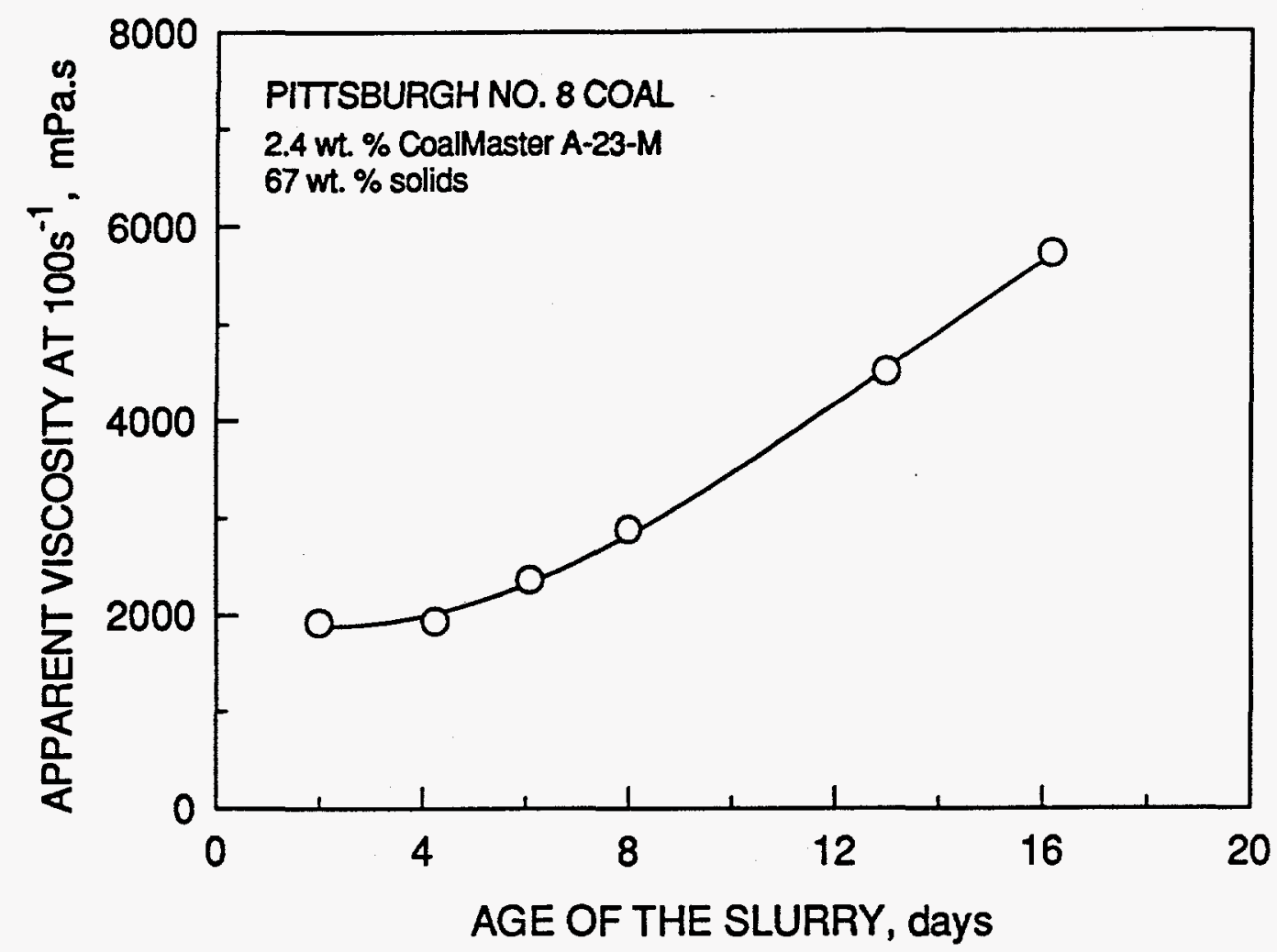

Figure 6.1. Effect of slurry aging on the viscosity of the coal-water slurry prepared with fines stored in air for nine months.

CoalMaster in the supernatant was measured by UV spectrophotometry. Figure 6.3 shows the plots of the CoalMaster concentration in the aqueous phase relative to that after two days of aging as well as the viscosity of the slurry relative to that after two days of aging, as functions of the age of the slurry. It can be observed from the plots given in this figure that the viscosity of the slurry increased with time with a corresponding decrease in the concentration of CoalMaster. The results of the experiments, conducted earlier to delineate the effect of CoalMaster concentration on slurry rheology, showed a similar reciprocal relationship between the viscosity of the freshly prepared slurry and the CoalMaster dosage. We also found in a separate experiment that the addition of freshly prepared ferric iron to an aqueous CoalMaster solution results in the formation of an insoluble precipitate. The decrease in CoalMaster concentration in the aqueous phase with time, in the aging experiment mentioned above, is most likely due to the complexation and precipitation of CoalMaster by iron. Since 

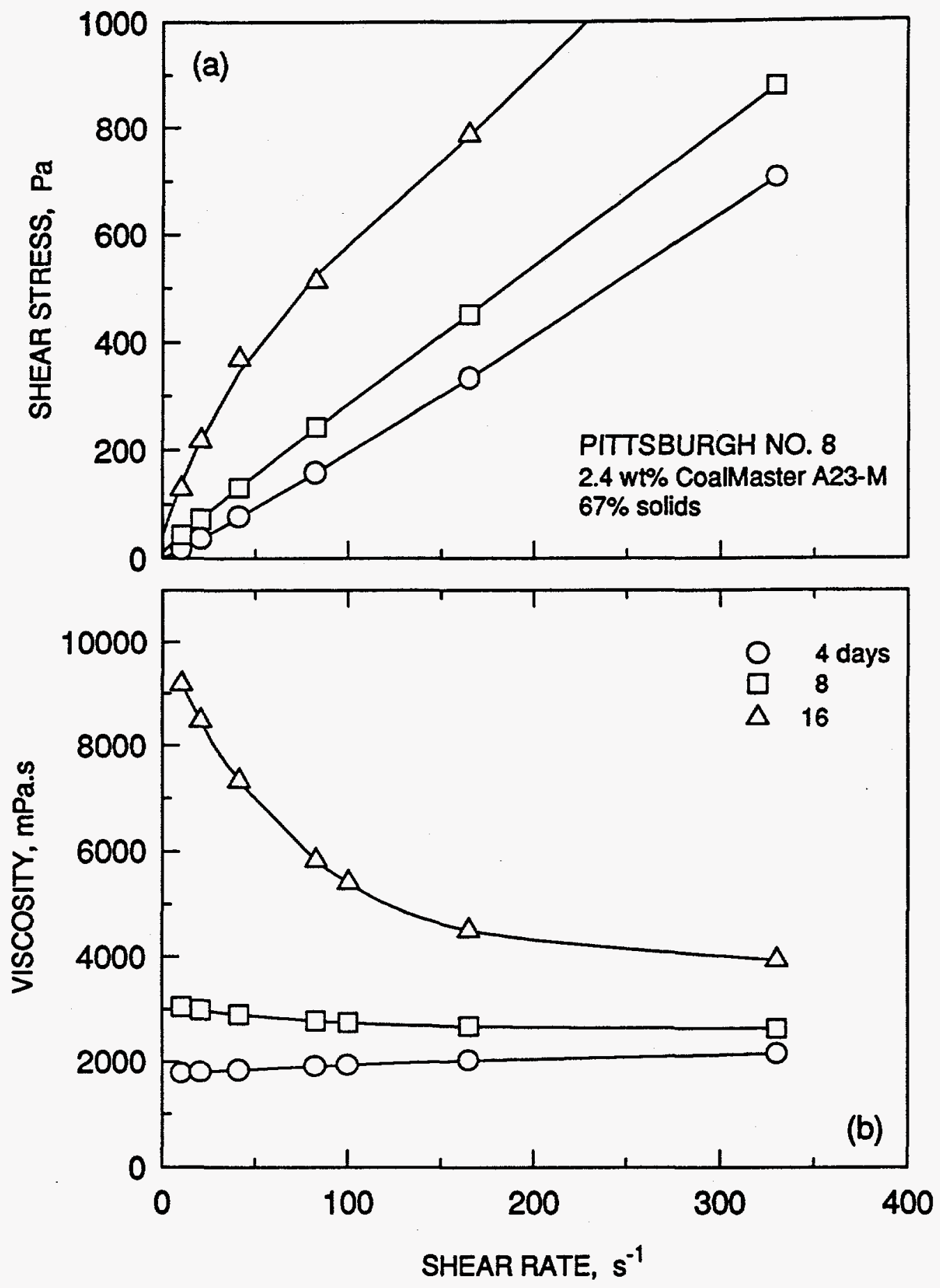

Figure 6.2. Effect of aging on the rheological behavior of coal-water slurry prepared with coal fines stored in air for nine months: a) flow curves, b) viscosity. 


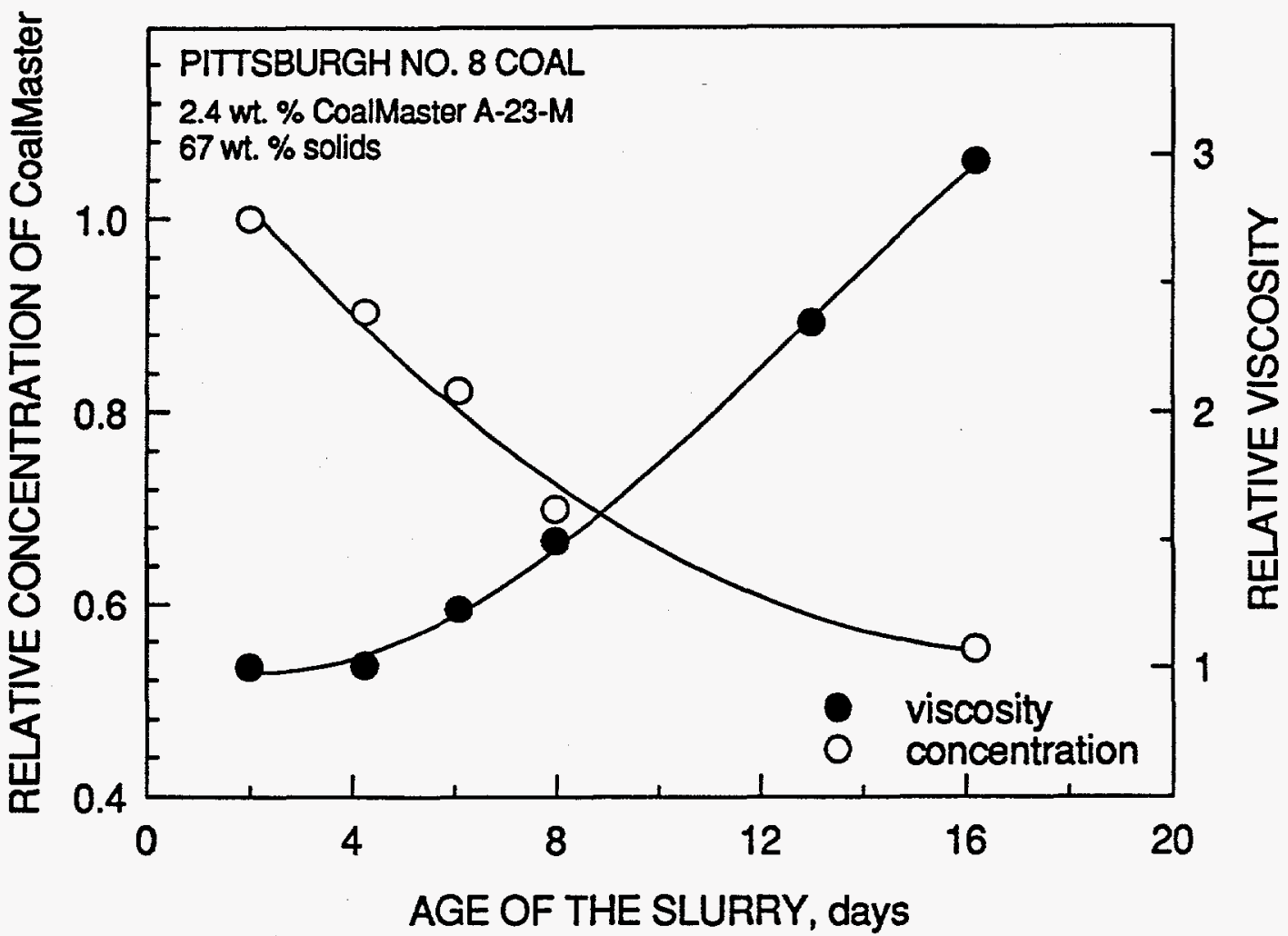

Figure 6.3. Effect of slurry aging on the concentration of CoalMaster A23-M in the aqueous phase and the viscosity of the coal-water slurry prepared with fines stored in air for nine months.

these results strongly suggest that iron is primarily responsible for the degradation of slurries over time, detailed investigations were carried out to identify the presence and the oxidation state of iron through spectroscopic analysis, establish how iron affects the surface characteristics of coal particles through electrokinetic measurements on aged slurries, and to determine the effect of iron removal (either by iron-complexing agents or by removal of pyrite by physical coal cleaning) on the aging behavior of slurries.

\section{Spectroscopic Studies of Iron Interaction with Complexing Reagents}

The nonionic iron-complexing reagent used in this investigation, 2,3-dihydroxypyridine (DHP) forms colored complexes with iron, which enabled us to follow whether any iron is present in the leachate from the CWS. The Fe(II) - DHP complex showed absorption maxima 
(UV/VIS spectra) at 418 and $516 \mathrm{~nm}$, and the Fe(III) - DHP complex had absorption peaks at 418 and $590 \mathrm{~nm}$. A Fe(III) -DHP complex prepared from the iron solution $(0.003 \mathrm{M})$ was left overnight to hydrolyze and showed absorption peaks at 418 and $546 \mathrm{~nm}$.

The Iron - DHP complex prepared from coal supernatant extracted from the CWS which had not been washed showed absorption peaks at 418 and $590 \mathrm{~nm}$, indicating the presence of both $\mathrm{Fe}^{2+}$ and $\mathrm{Fe}^{3+}$ cations. Surprisingly, no iron was observed in the supernatants from CWS which had been washed with nonionic or anionic reagents even though these slurries exhibited significantly increased viscosities after three weeks of storage. Centrifuging the samples of those CWS after dilution with aqueous solutions of DHP indicated that some iron was present on the coal surface. Iron-DHP complexes obtained by that procedure showed absorption maxima at 418 and $540 \mathrm{~nm}$, indicating the presence of some $\mathrm{Fe}^{2+}$ (small peak) and a significant amount of hydrolyzed iron species $\left(\mathrm{Fe}(\mathrm{OH})^{2+}\right.$ and $\mathrm{Fe}(\mathrm{OH})_{2}{ }^{+}$) (large peak at $540 \mathrm{~nm}$ ). Apparently, no unhydrolyzed ferric -DHP complex was present.

Similar experiments were performed with another iron-complexing reagent, phenantroline, which forms an orange complex with $\mathrm{Fe}^{2+}$ and colorless complex with $\mathrm{Fe}^{3+}$. We found that the coal leachate contained both $\mathrm{Fe}^{2+}$ and $\mathrm{Fe}^{3+}$ species (adding the reducing reagent $\mathrm{NH}_{2} \mathrm{OH} * \mathrm{HCl}$ almost doubled the absorbance at the orange peak maximum).

\section{Effect of Iron on the Electrokinetic Behavior of Pittsburgh No. 8 Coal}

The rheology of concentrated suspensions is controlled by interpartice forces, the particle size and size distribution, and the fraction of free water in the suspension. For charged particles, particle-particle interaction is primarily governed by the surface charge characteristics of the particles. To study this in more detail, the effect of aging on the surface charge of coal particles was investigated by means of electrokinetic measurements. For these studies, one gram of the $\mathrm{CW}$ slurry was first diluted to $100 \mathrm{ml}$ and resuspended by ultrasonication. The suspension was further diluted one-hundredfold to achieve the 10,000 


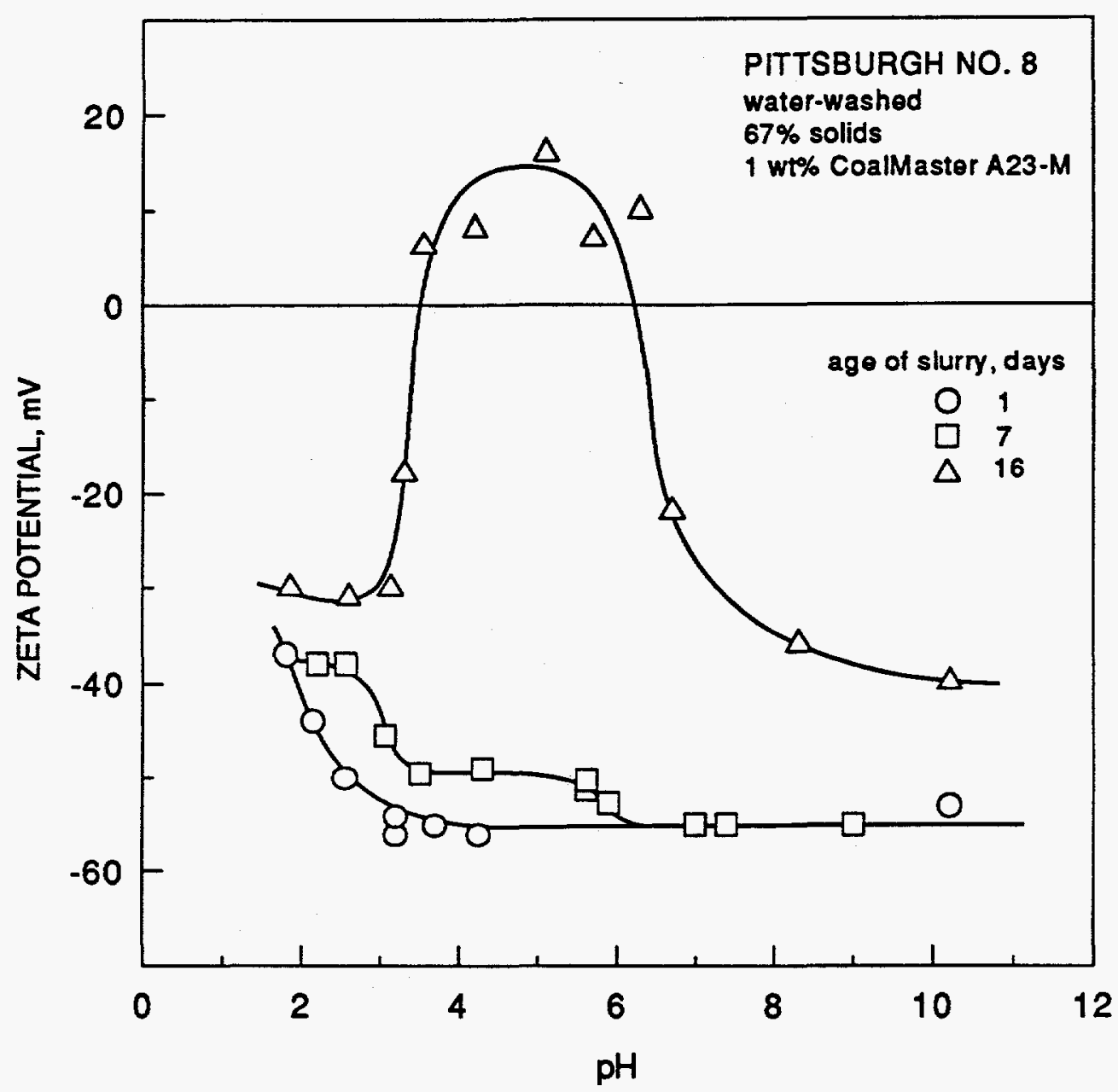

Figure 6.4. Effect of aging of coal-water slurry, prepared with water-washed coal and $1 \mathrm{wt} \%$ CoalMaster, on the zeta potential of coal particles.

times dilution necessary to perform the electrokinetic studies with the ZetaMeter 3.0 apparatus. Three coal-water slurries, each containing $67 \mathrm{wt} \%$ solids, prepared with waterwashed coal, floated coal, and untreated coal were used for the study of the effect of aging on the electrokinetic behavior of coal particles. The dispersant dosage was $2.4 \mathrm{wt} \%$ CoalMaster for the slurry prepared with the untreated coal and $1 \mathrm{wt} \%$ for the others.

Figures 6.4 to 6.6 show the effect of aging on the zeta potential of the coal particles in the three slurries. As can be seen from the plots given in Figures 6.4 and 6.5, the addition of CoalMaster to coal from which iron has been partially removed renders the particles highly 


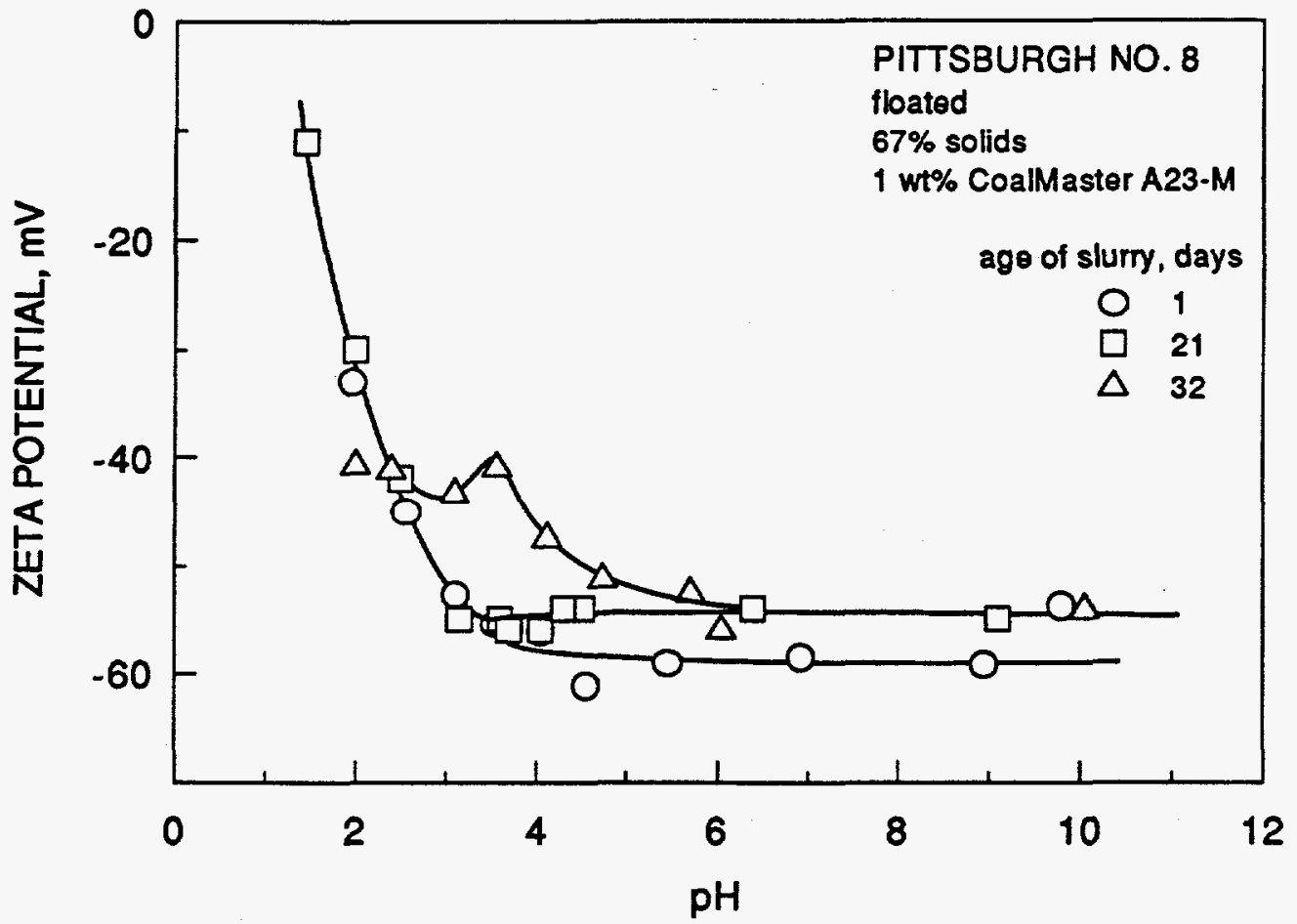

Figure 6.5. Effect of aging of coal-water slurry, prepared with floated coal and 1 wt\% CoalMaster, on the zeta potential of coal particles.

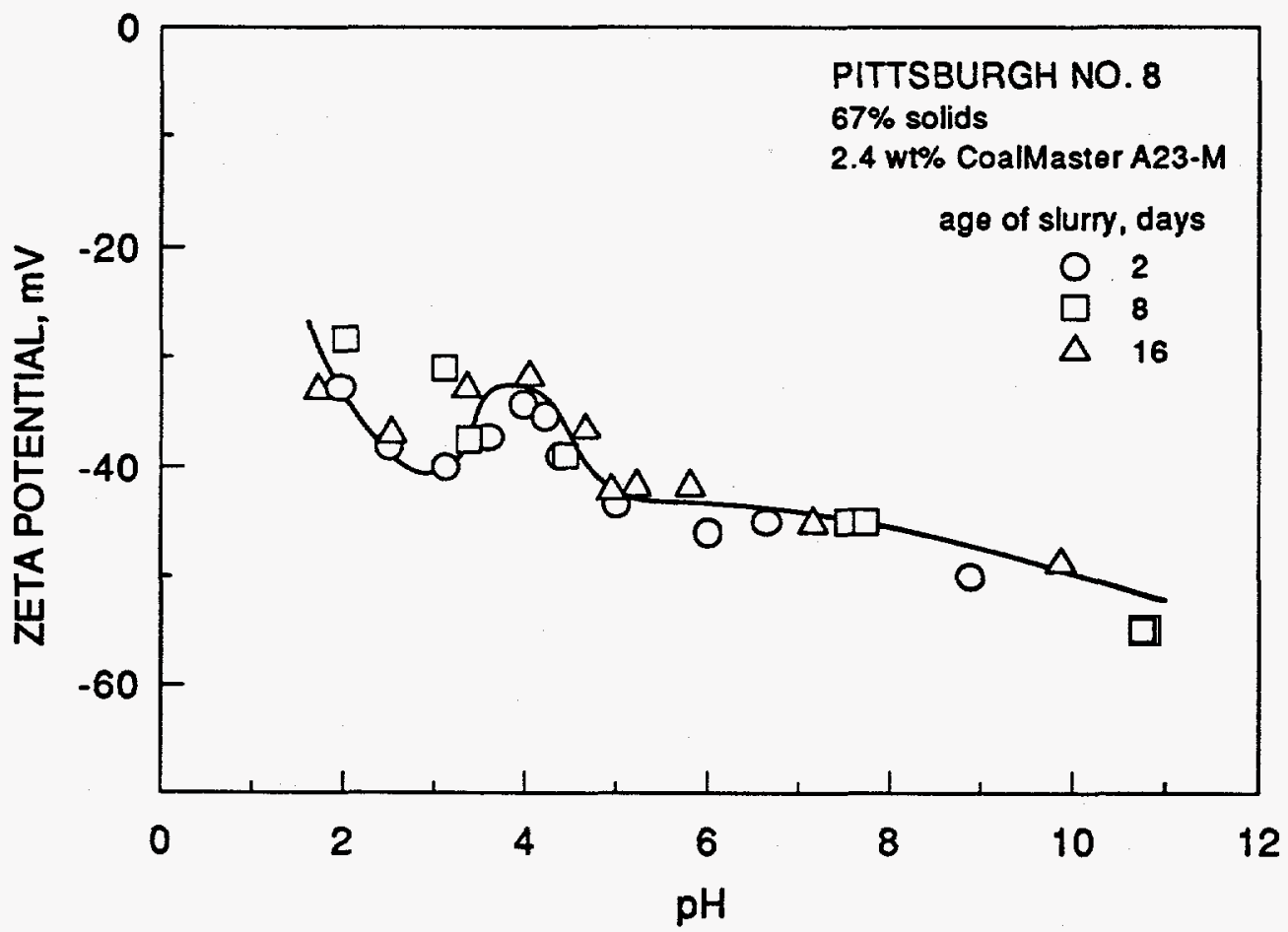

Figure 6.6. Effect of aging of coal-water slurry, prepared with untreated coal and 2.4 wt\% CoalMaster, on the zeta potential of coal particles. 


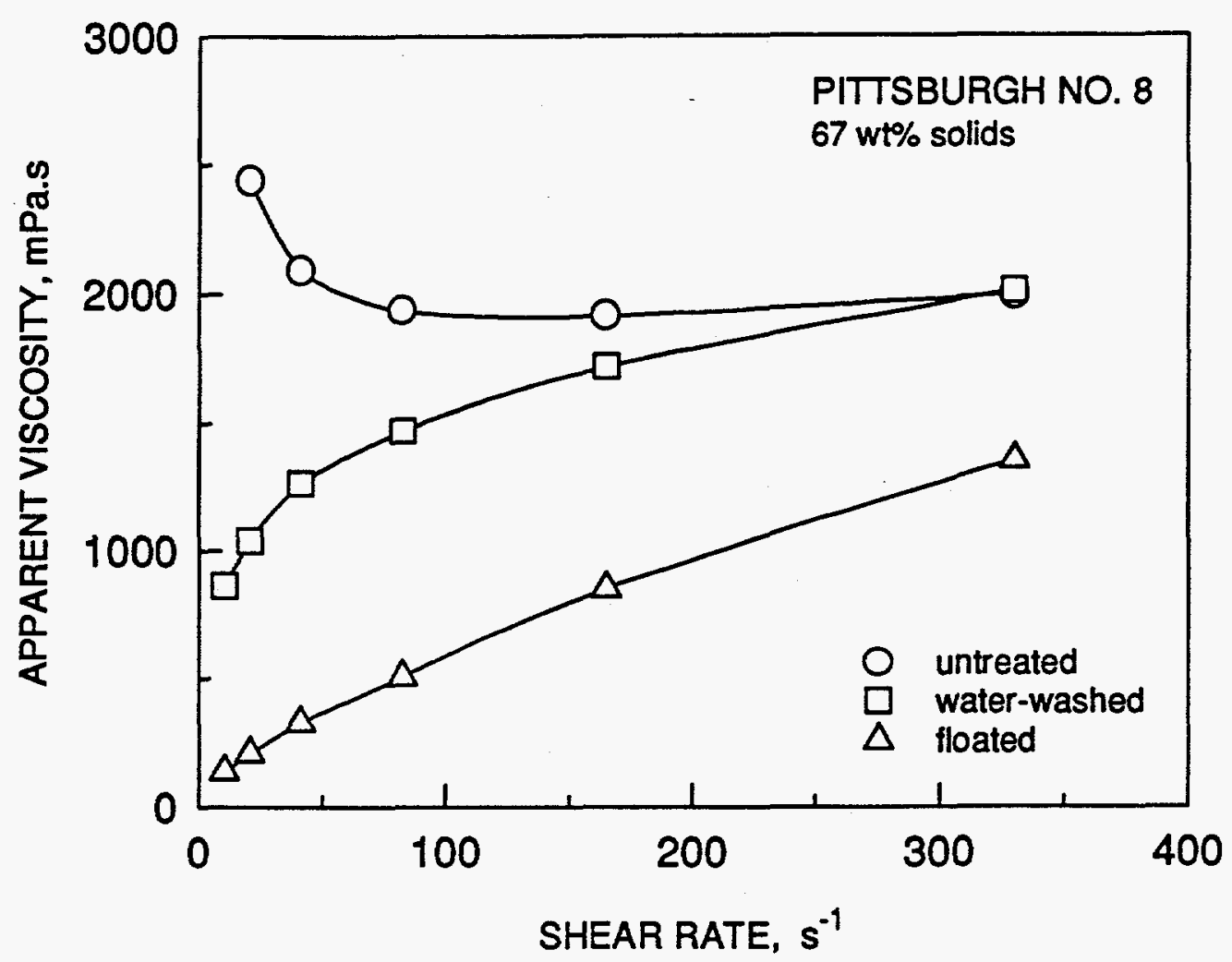

Figure 6.7. Apparent viscosity as a function of shear rate of the slurries prepared with untreated coal, water-washed coal, and floated coal.

negatively charged. This implies that the slurries would be well-dispersed, at least to start with, and is supported by the viscosity measurements. The two slurries exhibited lower apparent viscosities relative to that of the slurry prepared with untreated coal, and were slightly dilatant in nature, which is a charateristic of a well-dispersed slurry. The slurry prepared with untreated coal, on the other hand, was mildly thixotropic, a characteristic of weakly flocculated system and consistent with the zeta potential measurements. The apprent viscosity plots of the three slurries, as a function of shear rate, are given in Figure 6.7.

There was virtually no change in the zeta potential of the coal samples for at least a week. After two weeks, however, as can be seen from the plots given in Figure 6.4, the washed coal particles showed a less negative zeta potential over the entire $\mathrm{pH}$ range. Moreover, at pHs between 3 and 6 , the zeta potential actually became positive for these particles. The two isoelectric points observed in the acidic $\mathrm{pH}$ range strongly suggest that 


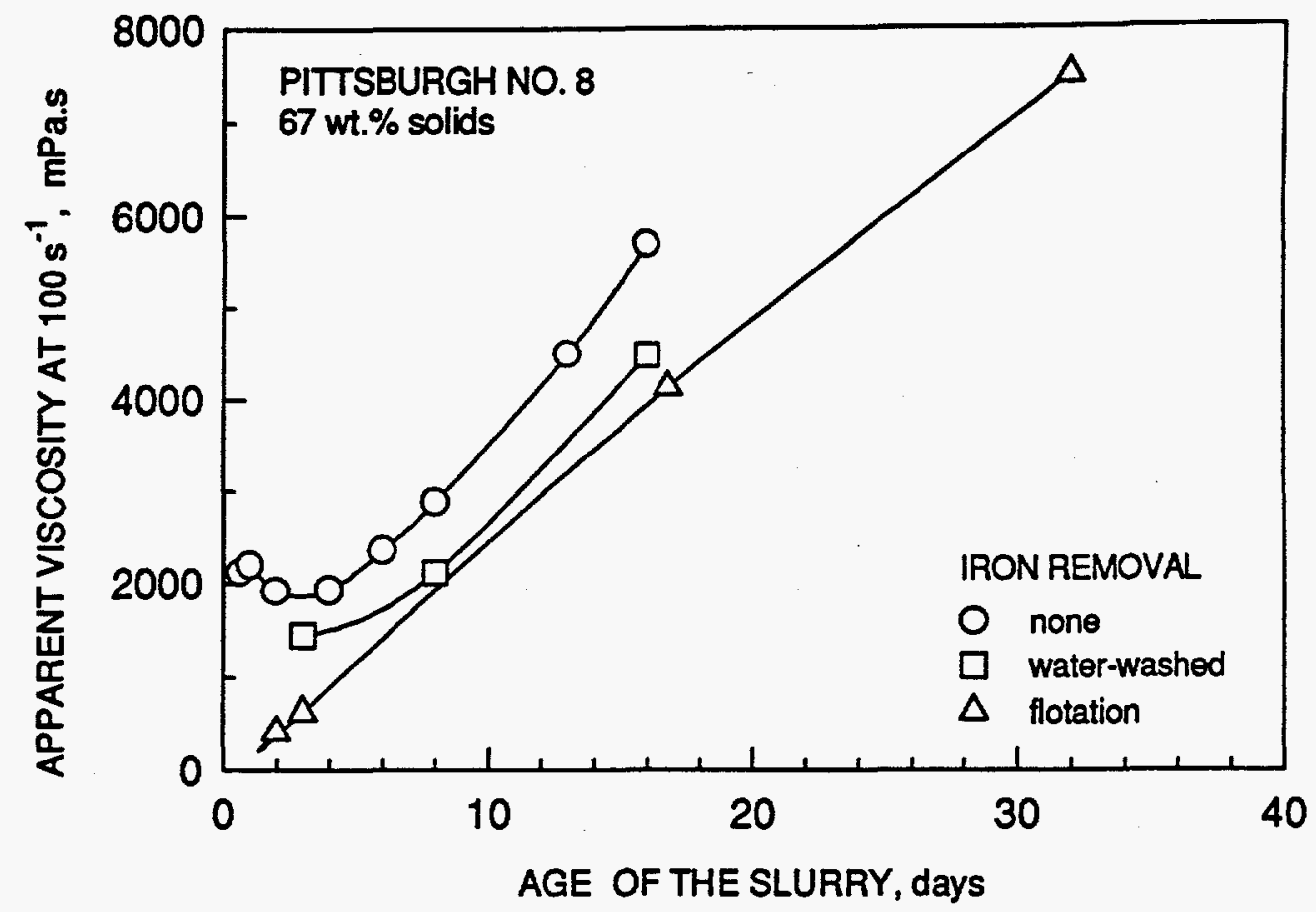

Figure 6.8. Effect of aging on the apparent viscosity of the coal-water slurries prepared with untreated, water-washed, anf floated coal fines.

hydrolyzed $\mathrm{Fe}^{3+}$ species are the cause of this behavior. A similar lowering, but of a much smaller of magnitude, of the zeta potential in this $\mathrm{pH}$ range was observed for particles contained in the freshly prepared slurry of the untreated coal sample. The zeta potential in this case, though, remained unchanged with the age of the slurry, as indicated by the results given in Figure 6.5. In the case of slurries prepared with the floated coal sample, there was practically no change in the zeta potential behavior for the first three weeks (Figure 6.6). Electrokinetic measurements carried out after 32 days, however, did show a small decrease in the surface charge for this sample over the same $\mathrm{pH}$ range. This decrease is again probably due to the adsorption of hydrolyzed $\mathrm{Fe}^{3+}$ species.

The change in the apparent viscosities of the three samples with aging are plotted in Figure 6.8. The aging behavior of slurries prepared with water-washed coal is consistent with the effect of aging on the surface charge of coal particles. Although the surface charge behavior as a function of $\mathrm{pH}$ of untreated coal particles did not change significantly with time, 
the $\mathrm{pH}$ of this slurry dropped to 1.8 after 8 days. At this $\mathrm{pH}$, the zeta potential of the particles would be about $-20 \mathrm{mV}$, a condition suffcient for coagulation and consequent increase in the viscosity of the slurry. On the other hand, while the surface charge of floated coal particles remained highly negative, even after nearly four weeks of aging, slurries prepared with such coal also exhibited an increase in apparent viscosity over time. The results indicate that in presence of a high concentration of iron and/or low dosage of CoalMaster the surface charge of the coal particles probably controls the rheological properties of aged slurries. In all other cases, the surface charge may have at best a secondary effect on the rheology of aged slurries. The degradation of the rheological behavior of such systems on aging may be controlled by the free water content of the slurries, as will be discussed later in detail.

\section{CONTROL OF AGING BEHAVIOR OF COAL-WATER SLURRIES}

So far we have established through spectroscopic and electrokinetic stduies that a significant amount of iron may be present in aged coal-water slurries which, in turn, can affect their rheological behavior. Release of iron through oxidation of the pyrite in coal and its subsequent hydrolysis leads to a substantial lowering of the $\mathrm{pH}$ of the slurry and zeta potential of the particles. Hydrolyzed iron species may adsorb on coal surfaces and lower the surface charge of the particles. Iron may also form a precipitate with CoalMaster, the polyelectrolyte used as the dispersant. All these factors contribute to the observed increased viscosity of slurries upon aging. The question that arises concerns how might the aging behavior of slurries be controlled. To this end, we conducted several different studies: i) removal of soluble iron by washing the gorund coal with aqueous solutions of iron-complexing reagents prior to the preparation of slurries, ii) removal of liberated pyrite through flotation and/or physical cleaning (tabling), and iii) controlling the rate of oxidation of coal by controlling the rate of iron release through the addition of vaccum oil.

Since coal surface behavior can vary significantly depending on the degree of oxidation, a baseline was first established by studying the aging behavior of coal-water slurry 
prepared with untreated coal. The effect of treating coal for the control of the iron content of aged slurries was then evaluated against the baseline.

\section{Effect of Iron-complexing Reagents on the Rheology of Coal-Water Slurries}

Iron cations are released through pyrite oxidation in the +2 form and oxidize slowly to the +3 form, which subsequently hydrolyzes. This effect has two negative consequences with regard to the viscosity and stability of coal-water slurries. First, iron hydrolysis causes the $\mathrm{pH}$ to drift to the acidic region. It is well documented [42] that Coal Master A23-M dispersant works best in neutral or slightly basic media $(\mathrm{pH} 7-9)$; lowering the $\mathrm{pH}$ of the slurry decreases the reagent efficacy. Second, hydrolyzed iron (3+) species adsorb strongly on almost anything and flocculate suspended particles above a certain concentration, that is when the so-called critical coagulation concentration is reached.

A coal sample that has been stored in air for nine months was used for the study of the effect of soluble iron removal by complexation with reagents on the aging behavior of coalwater slurries. As reported earlier, this sample contained a considerable amount of oxidized iron in soluble form, and it was hoped that the effect of iron removal on the rheological behavior would be much more pronounced for slurries prepared with this coal, as compared with slurries prepared with freshly ground coal. An anionic iron-complexing agent, sulfosalicylic acid, and a nonionic iron-complexing reagent, 2,3-dihydroxypyridine, were used for the removal of soluble iron.

The procedure for washing the coal samples invloved the following steps. 125 grams of the ground coal sample was first mixed with 2 liters of distilled water in a 2-liter flotation cell, then $0.15 \mathrm{wt} . \%$ of iron-complexing reagent was added, and the slurry was agitated for 15 minutes. After agitation, the slurry was filtered and the coal was then dried in a mechanical convection oven at $40^{\circ} \mathrm{C}$ for 12 hours. A CWS containing $67 \mathrm{wt} \%$ solids and $2.4 \mathrm{wt} \%$ CoalMaster as dispersant was then prepared and aged in the enviromental shaker for various lengths of time. The shaker $\mathrm{pm}$ and the temperature were maintained at 200 and $20^{\circ} \mathrm{C}$, 


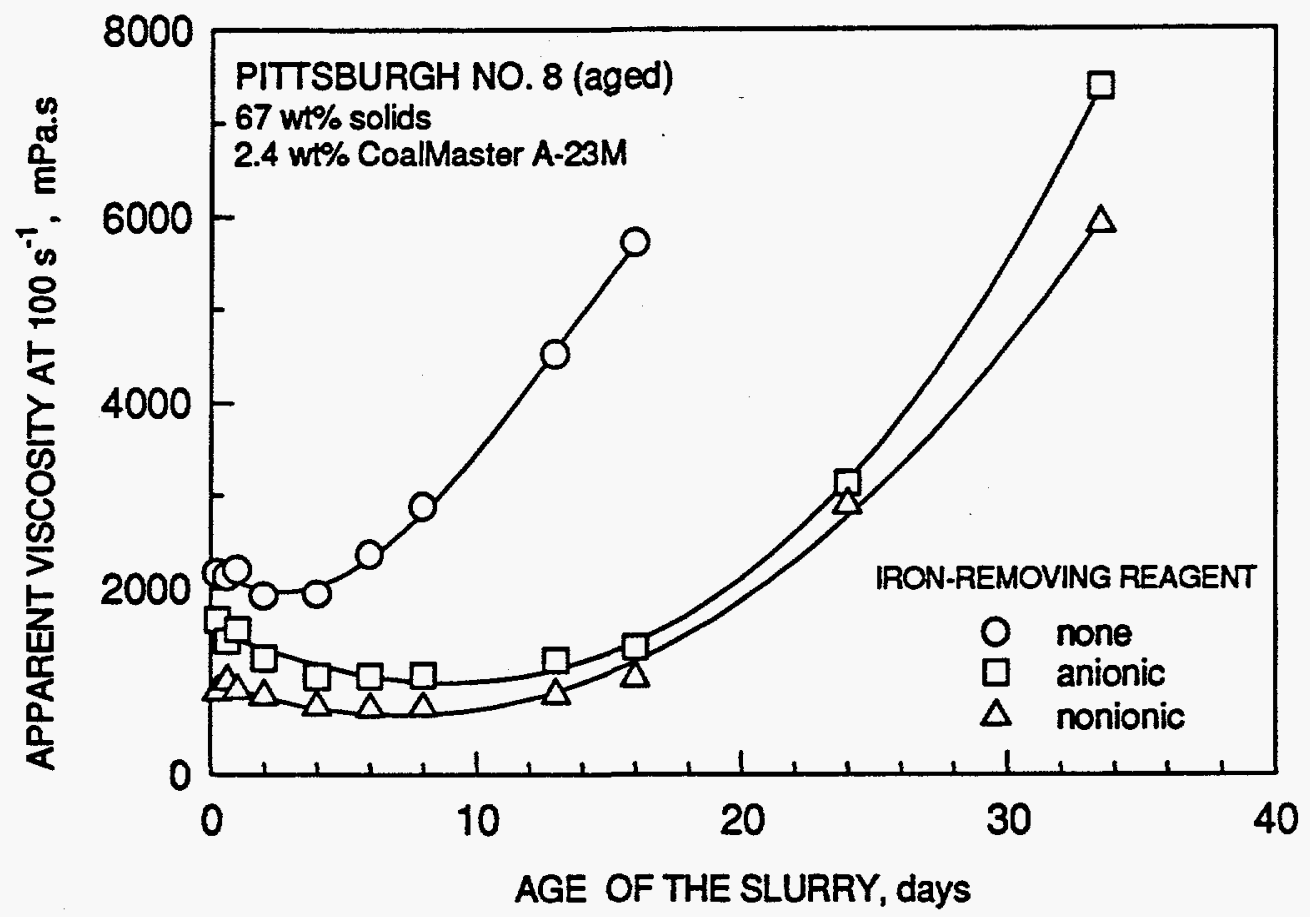

Figure 6.9. Effect of slurry aging on the viscosity of the coal-water slurry prepared with i) fines stored in air for nine months, ii) fines washed with anionic iron-complexing reagent, and iii) fines washed with nonionic iron-complexing reagent.

respectively. Figure 6.9 presents the apparent viscosity of CWS at $100 \mathrm{~s}^{-1}$ as a function of aging of the slurries after their preparation. The results presented in this figure show that unwashed coal had high initial viscosity (2000 mPa.s at $100 \mathrm{~s}^{-1}$ ), which doubled after 14 days. CWS prepared from coal samples washed with the anionic reagent exhibited a slightly lower initial viscosity $\left(1700 \mathrm{mPa} . \mathrm{s}\right.$ at $\left.100 \mathrm{~s}^{-1}\right)$. The initial viscosity further decreased during first 10 days of slurry aging to $1100 \mathrm{mPa}$.s at $100 \mathrm{~s}^{-1}$, which is an acceptable value. Further slurry aging increased the viscosity to $2000 \mathrm{mPa}$.s after three weeks and 5700 after four weeks. Similar results were obtained for the CWS prepared from coal washed with nonionic reagent but the initial viscosities were even lower (900 mPas at $\left.1000 \mathrm{~s}^{-1}\right)$. Viscosities of that particular slurry were acceptable even after three weeks of aging (1500 mPa.s at $\left.100 \mathrm{~s}^{-1}\right)$.

Figure 6.10 illustrates the effect of aging on the rheological behavior of a coal-water slurry prepared with untreated coal. Initially, the slurry was nearly Newtonian, with negligible 

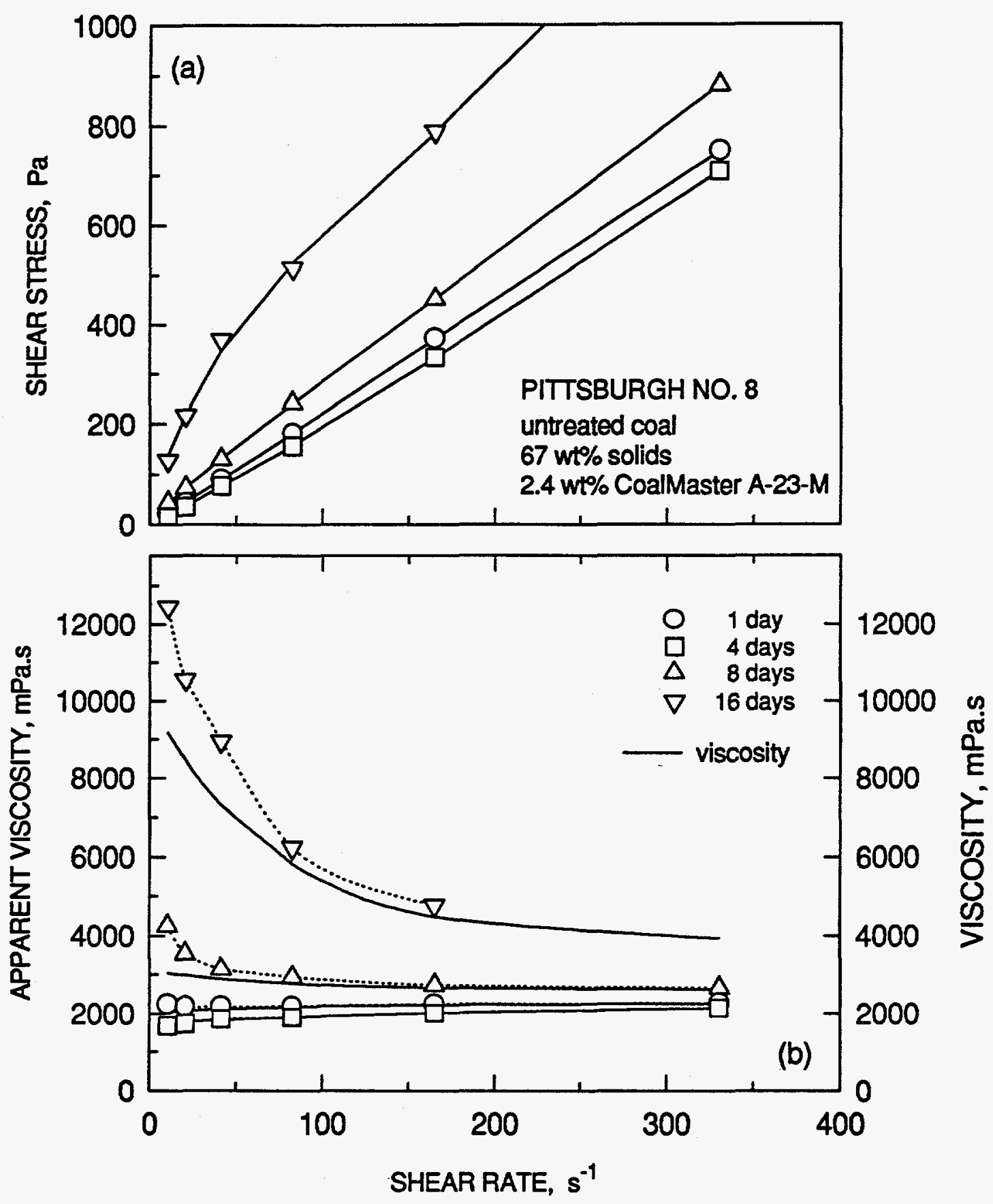

Figure 6.10. Effect of aging on the meological behavior of coal-water slurry prepared with coal fines stored in air for nine months: a) flow curves, b) true and apparent viscosity. 
yield stress. After eight days, however, the slurry exhibited slight shear thinning and a small yield stress. This behavior became rather pronounced after sixteen days. This strongly suggests that, initially, the slurry is weakly flocculated and becomes strongly flocculated with aging [42].

Different flow behaviors were observed for slurries prepared from coal which had been washed with the nonionic and anionic reagents. While the CWS prepared with fines washed with anionic reagent had higher viscosity compared to that of the slurry prepared with fines washed with nonionic reagent, the flow behavior of the two slurries was very similar. Both slurries showed no yield stress, even after three weeks of aging. Flow curves indicate shear thickening behavior (shear thickening is characteristic of well-dispersed systems). This suggests that iron present in the coal leachate and/or adsorbed on the surface is removed by this washing procedure. After one month, however, the viscosity of the slurry again increased significantly. Some yield stress and shear thinning behavior can be observed. As mentioned earlier, these are characteristic of flocculated slurries. No iron was observed in the leachate of these slurries, but washing the coal with iron-complexing reagents indicates the presence of the hydrolyzed iron (3+) species strongly adsorbed on the surface, as explained earlier in this section. Figure 6.11 shows the apparent viscosity and flow curves and their change with the age of coal-water slurry prepared with fines washed with nonionic reagent.

Figure 6.12 presents the $\mathrm{pH}$ of these slurries as a function of their age. The slurry prepared from unwashed coal had a low initial $\mathrm{pH}$ of 2.7 despite the addition of $0.7 \mathrm{ml}$ of 15 molar ammonium hydroxide. The $\mathrm{pH}$ of this slurry showed a logarithmic decrease with time, dropping from 2.7 to 1.8 in eight days, indicative of a continuous oxidation of pyrite and release of iron into the aqueous phase. Our zeta potential measurements indicate that CoalMaster is not an effective dispersant at such low $\mathrm{pH}$ s. The slurries prepared with washed coals, on the other hand, had relatively higher starting $\mathrm{pH}$ 's, and showed practically no change in $\mathrm{pH}$ for the first week or so. However, from then onwards, there was a logarithmic decrease 

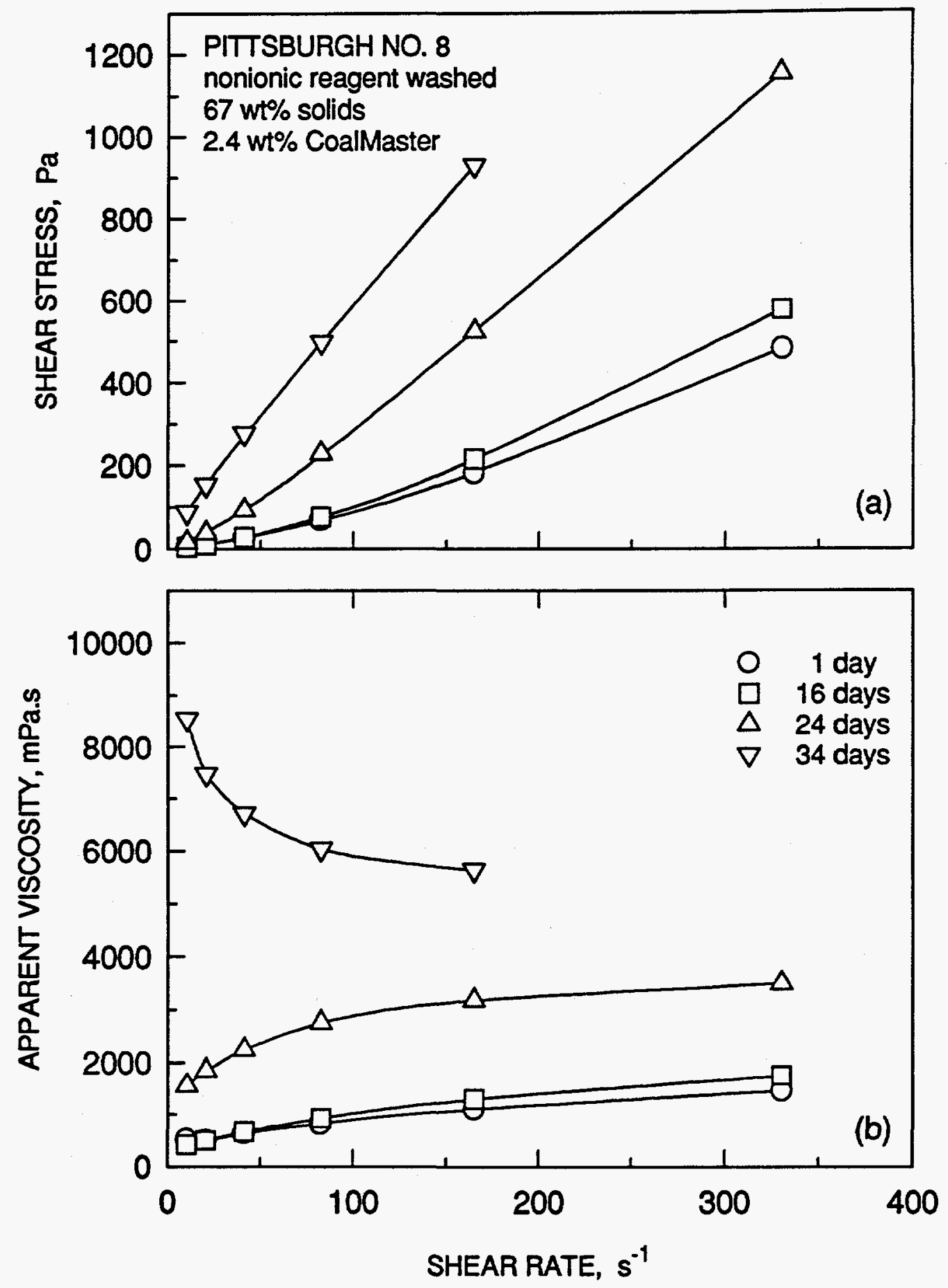

Figure 6.11. Effect of aging on the meological behavior of coal-water slumy prepared with coal fines washed with nonionic reagent: a) flow curves, b) apparent viscosity. 


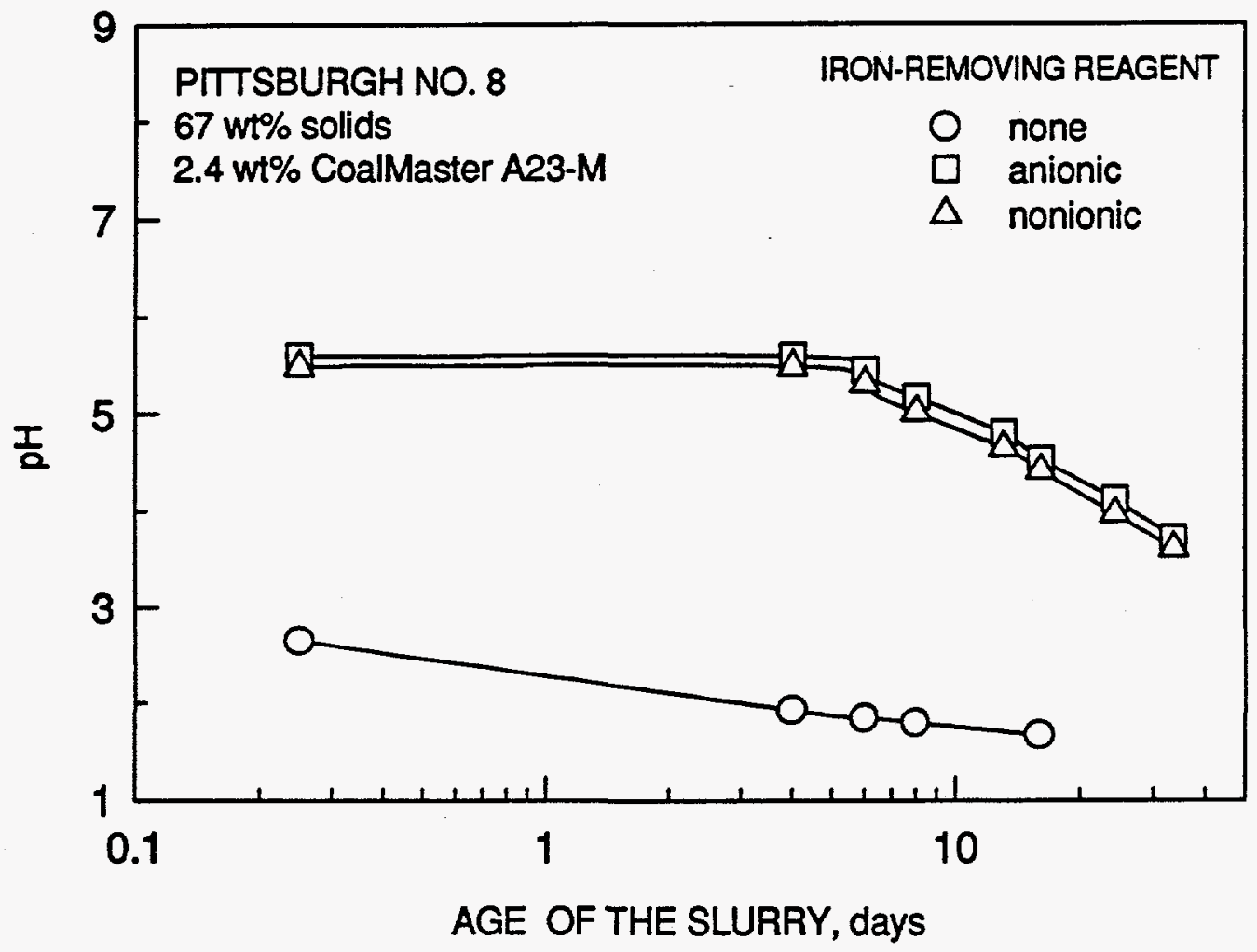

Figure 6.12. Effect of slurry aging on the $\mathrm{pH}$ of the coal-water slurries prepared with fines stored in air for nine months, fines washed with anionic iron-complexing reagent, and fines washed with nonionic iron-complexing reagent.

in the $\mathrm{pH}$ of these slurries as well, again due to the oxidation of pyrite and hydrolysis of ferric iron. While there is no one-to-one correspondence between the change in $\mathrm{pH}$ and the degradation of the rheological behavior of the slurries, the drop in $\mathrm{pH}$ over time provides indirect evidence of the role of iron in the degradation of the slurry rheology.

The results, discussed above, show that removal of soluble iron from coal prior to the preparation of the slurry can significantly improve the aging behavior of the slurry. However, there will be a continouous release of iron into the slurry by oxidation of the pyrite contained in coal. In the foregoing experiments, the iron-complexing reagent was added during the coal washing step, but it was subsquently removed as the washing and filtration process proceeded. Therefore, in yet another experiment, we investigated the aging behavior of a slurry prepared with unwashed coal to which $0.5 \mathrm{wt} \%$ nonionic iron-complexing reagent was added. The 


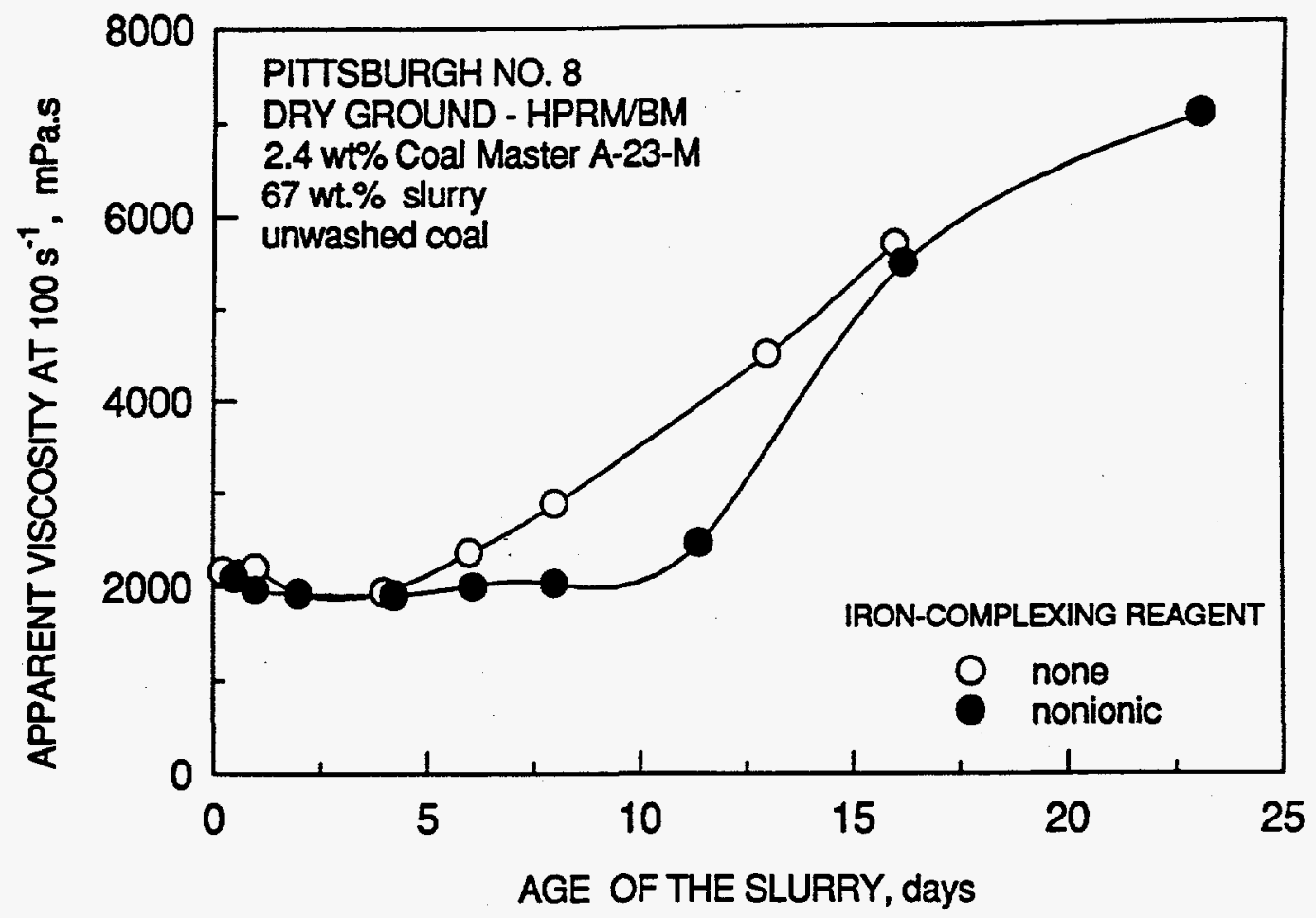

Figure 6.13. Effect of complexation of soluble iron by nonionic iron-complexing reagent on the aging behavior of coal-water slurry prepared with fines stored in air for nine months.

objective of this particular experiment was to investigate whether the addition of ironcomplexing agent could either prevent or slow down the degradation of the rheological behavior of such slurries.

Figure 6.13 shows the effect of complexation of soluble iron in the aqueous phase of the slurry on the apprent viscosity of the slurry over time. As can be seen from the figure, while the initial viscosities of both the slurries were nearly identical, iron-complexation resulted in a marked improvement in the aging behavior of the slurry over the first twelve days. Beyond this, however, there was a sharp increase in the viscosity of the slurry, and after sixteen days the viscosities of the two slurries were almost the same. The probable reason for this increase is that after twelve days the iron concentration in the aqueous phase was greater than that which could be complexed by the reagent. The sharp increase in the viscosity is associated with a transition in the rheological behavior of the slurry from near-Newtonian, 

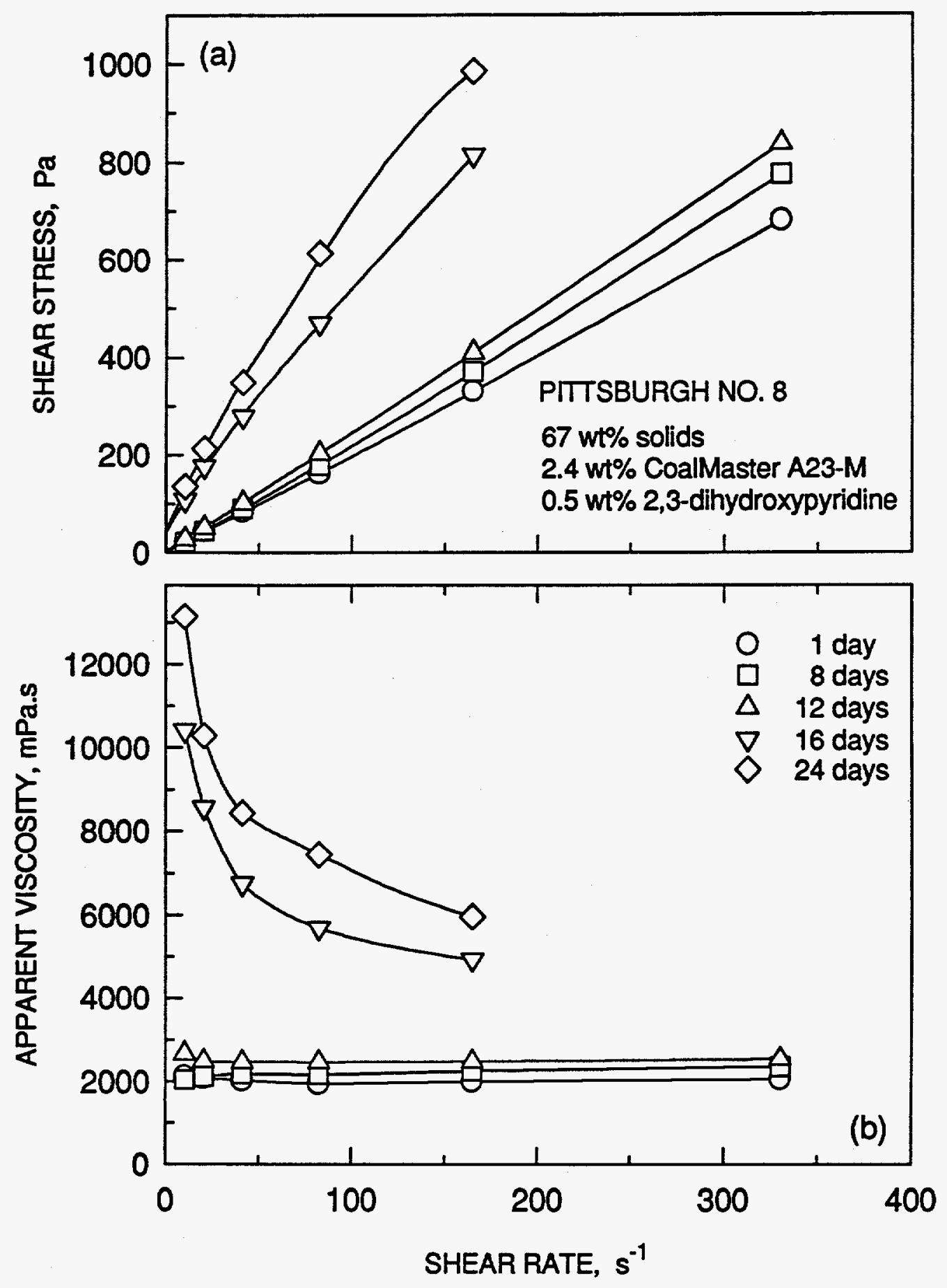

Figure 6.14. Effect of complexation of soluble iron on the aging behavior of the rheological properties of coal-water slurry: a) flow curves, b) apparent viscosity. 
with negligible yield stress, to thixotropic, with significant yield stress, as shown in Figure 6.14. At this point, the rheological behavior of the two slurries was virtually identical. Nevertheless, the study indicates that even when soluble iron is present, the addition of a sufficiently high dosage of iron-complexing reagent may either completely prevent or definitely retard the degradation of the rheological properties of slurries over time.

\section{The Effect of Coal Cleaning on the Rheology of Slurries}

The results of our research, presented above, showed that the presence of soluble iron adversely affects both the short-term as well as the long-term characteristics of coal-water slurries. We observed that removal of iron by washing coal with water, both in the presence and absence of iron-complexing reagents, prior to preparing the slurries at least temporarily improves the aging stability of the slurries. Since pyrite contained in the coal is the source of the iron, it was thought that a partial removal of pyrite by either gravity separation or by froth flotation could slow down the degradation of the slurry rheology. Accordingly, clean coal samples were prepared by gravity separation (using a standard laboratory Wilfley table), and also by flotation.

Preparation of clean coal by gravity separation: A $2.0-\mathrm{kWh} / \mathrm{t}$ high-pressure roll mill product was ground in our 10-inch torque ball mill for one minute, essentially for deagglomerating the roll mill product. A 25-wt\% coal-water suspension was prepared from the mill discharge which was then cleaned using the Wilfley table. The concentrate from the tabling operation was dried and then ground in the 8-inch ball mill to $95 \%$ minus 200-mesh.

Preparation of clean coal by flotation: The same roll mill product was ground in the 8-inch ball mill to $95 \%$ minus 200-mesh. A part of the mill discharge was then floated in a 2-liter Denver cell for 3 minutes, with GH4 as collector and MIBC as frother. The flotation concentrate, after filtering and drying, constituted the clean coal sample. 
Table 6.1. Composition of as-ground and cleaned coal samples

\begin{tabular}{|c|ccc|}
\hline Sample & Combustible matter & Ash & Density, ${\mathrm{g} . \mathrm{cm}^{-3}}^{-3}$ \\
\hline Ground only & 88.15 & 11.85 & 1.43 \\
Ground and floated & 91.75 & 8.25 & 1.35 \\
Tabled and ground & 94.63 & 5.37 & 1.30 \\
\hline
\end{tabular}

The proximate analysis (dry basis) and densities of the three fine coal samples are given in Table 6.1. These results show that both cleaning methods result in a significant reduction in the ash content, with gravity separation resulting in removal of more than half the mineral matter.

Each of the fine samples was used to prepare a $67 \mathrm{wt} \%$ slurry, with $3 \mathrm{wt} \%$ CoalMaster A-23M as the dispersant. Rheology measurements on these slurries showed that cleaning of the coal results in a slight lowering of the viscosity. The benefits of cleaning are, however, more apparent if we examine the change in the combustible matter content and the volumetric solids content of the slurries as a result of cleaning. Gravity separation results in nearly 5\% increase in the combustible matter content and a $3 \%$ increase in the solids content on a volume basis. The results are summarized in Table 6.2.

The study of the aging behavior of these slurries showed that partial removal of pyrite by coal cleaning slows down appreciably the degradation of their rheological behavior over time. The apparent viscosity of slurries prepared with freshly ground coal, floated coal, and

Table 6.2. Effect of coal cleaning on the composition and rheology of coal-water slurries

\begin{tabular}{|c|cccc|}
\hline Sample & $\begin{array}{c}\text { App. viscosity at } \\
100 \mathrm{~s}^{-1}, \mathrm{mPa} . \mathrm{s}\end{array}$ & $\begin{array}{c}\text { Wt\% combustible } \\
\text { matter }(\mathrm{CM})\end{array}$ & $\begin{array}{c}\text { Vol. \% } \\
\text { solids }\end{array}$ & $\begin{array}{c}\text { Wt\% CM per } \\
\text { unit volume }\end{array}$ \\
\hline Ground only & 2520 & 57.2 & 58.6 & 71.7 \\
Ground \& floated & 2340 & 59.9 & 60.1 & 72.5 \\
Tabled \& ground & 2390 & 62.1 & 61.0 & 73.5 \\
\hline
\end{tabular}




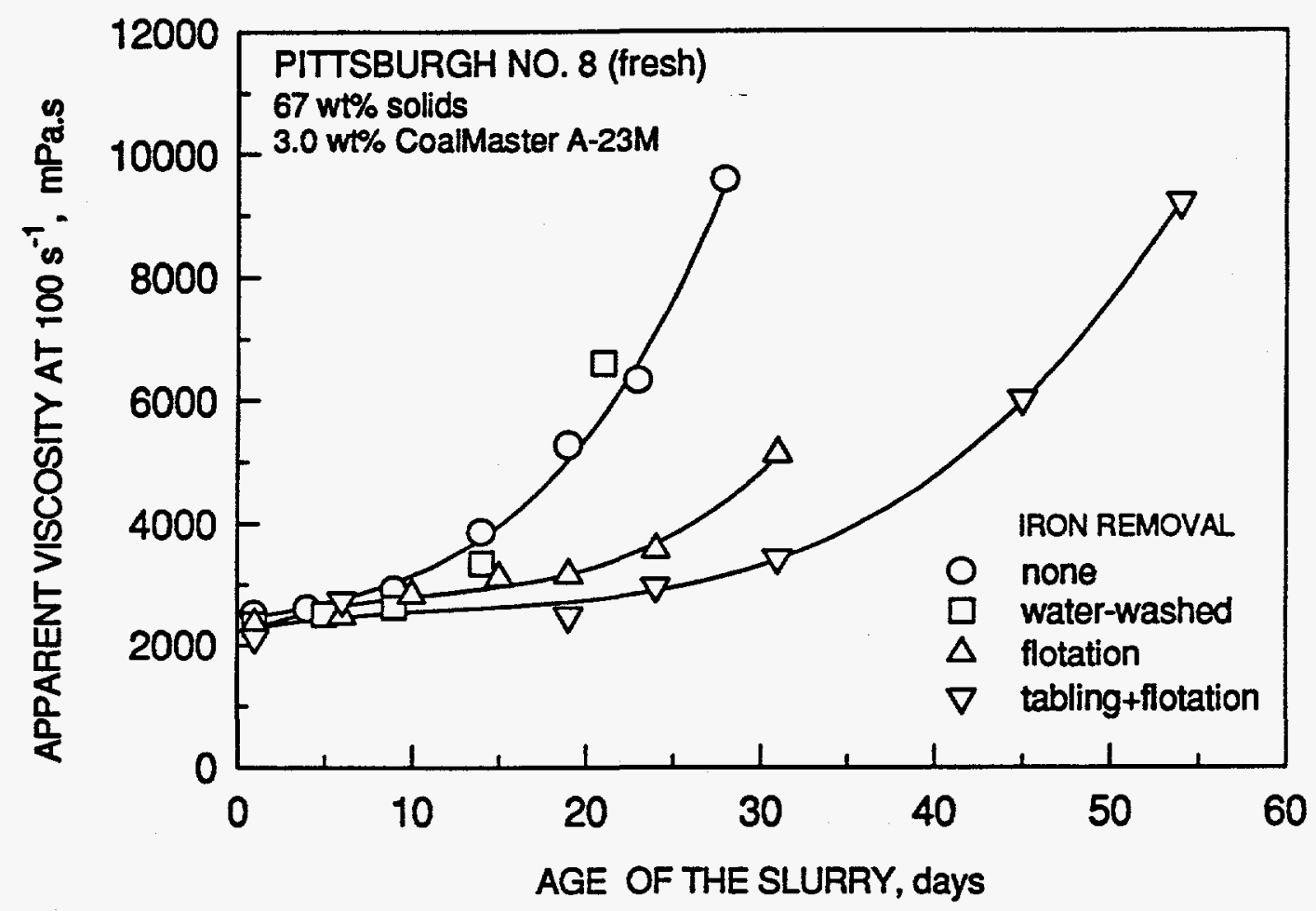

Figure 6.15. The effect of coal cleaning on the aging behavior of coal-water slurries.

tabled-and-floated coal, as a function of slurry age is shown in Figure 6.15. Both tabling and flotation involves the use of a large amount of water, and it is likely that most of the soluble iron would be removed from the coal during these processes. Therefore, the aging behavior of a fourth slurry prepared with water-washed coal was also investigated and the plot of the apparent viscosity of this slurry as a function of its age is included in Figure 6.15. As can be seen from the figure, the apparent viscosity of the freshly prepared slurries were nearly the same. Moreover, the aging behavior of the slurry prepared with water-washed coal is only marginally better than that of the slurry prepared with untreated coal. Both these indicate that this coal sample was relatively unoxidized and free from soluble iron.

The aging behavior of the slurry prepared with freshly ground coal is similar to that of the slurry prepared with the coal sample that was stored in air for nine months. The rate of degradation of this slurry was slower, however, probably because of its lower soluble iron content. It is apparent from Figure 6.15 that washing of freshly ground coal prior to the 
preparation of slurries did not have any effect on their aging behavior. Partial removal of pyrite, on the other hand, dramatically improves the aging behavior of the slurries. For instance, in about eleven days the apparent viscosity of the slurry prepared with untreated coal increased to $3200 \mathrm{mPa}$.s from an initial value of approximately $2400 \mathrm{mPa}$.s. Similar increase in the viscosity of slurries prepared with floated coal and tabled-and-floated coal took 21 and 30 days, respectively. Cleaning coal by gravity separation followed by flotation resulted in maximum improvement in the aging behavior of slurries. Unfortunately, even these slurries exhibited an increase of nearly two and a half times in the apparent viscosity after 45 days and almost a fourfold increase after 54 days.

\section{Control of Aging Behavior of Slurries by the Addition of Vacuum Oil}

Cleaning coal for the removal of pyrite results in a marked improvement in the aging behavior of slurries. The viscosities of these slurries remain stable for an extended period of time. Eventually, however, these slurries also exhibit an unacceptable increase in viscosity. The primary reason for the increase is the continuous oxidation of the coal particles in the slurry. We had shown earlier that co-addition of vacuum oil, along with sodium hexametaphosphate, to the slurry can result in a 50 percent decrease in suspension viscosity. This decrease is presumably because of the lowered interaction of water with the coal surface because of the addition of oil. The degradation of the rheological properties of slurries is mainly due to loss of free water through imbibition of water in the pores of the coal particles over time and the leaching of iron from pyrite in coal by water and oxygen. Since the addition of oil reduces the coal-water interaction, it was expected that oil additions should have some bearing on the aging behavior of coal-water slurries. Aging studies indicate that oil addition indeed improves the long-term aging behavior of coal-water slurries. Apparent viscosities of slurries prepared with and without the addition of vacuum oil are plotted as a function of their

age in Figure 6.16. The apparent viscosity of slurries prepared without oil increased about 50 percent in two weeks, while slurries containing oil showed only a little over ten percent 


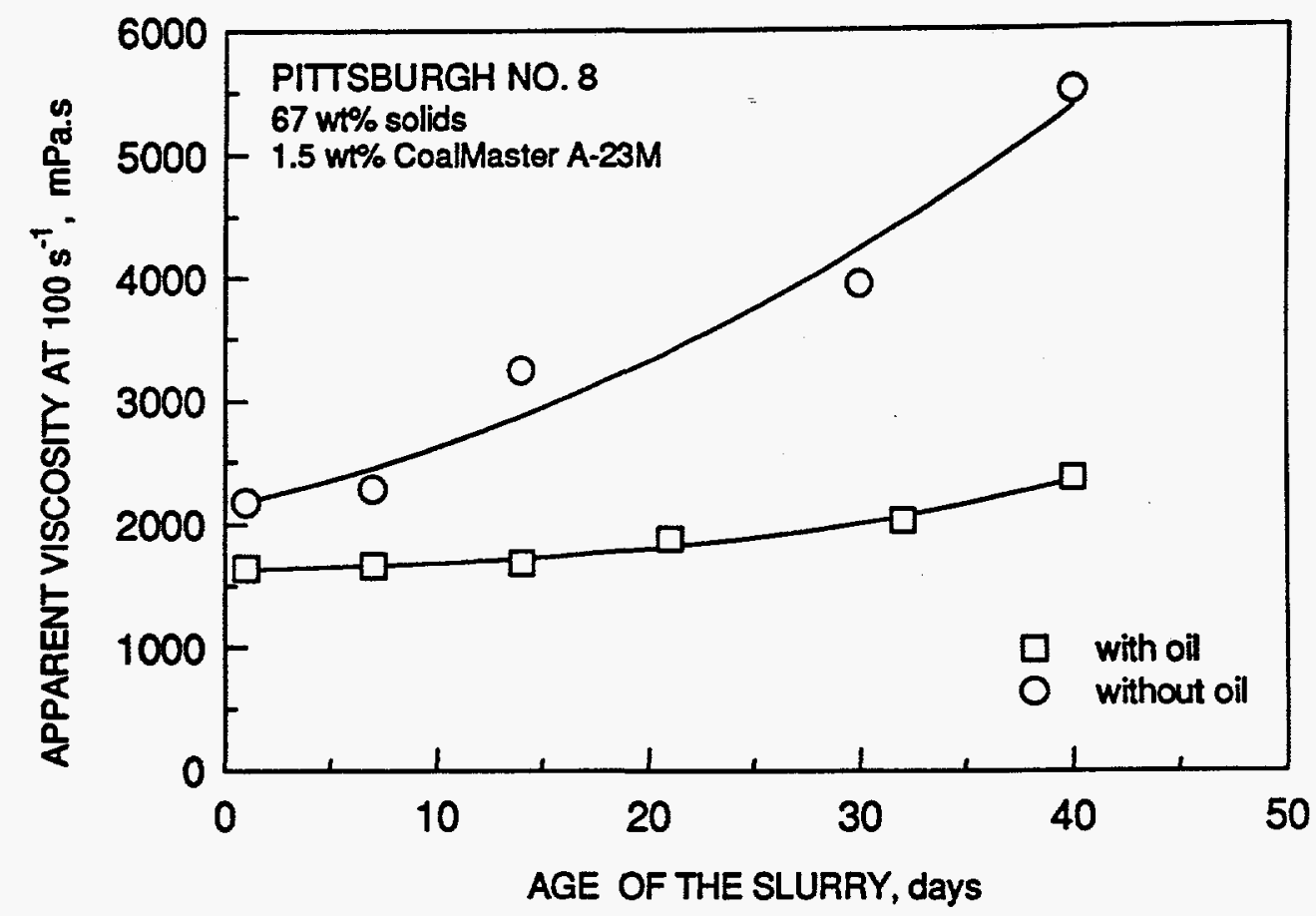

Figure 6.16. The effect of co-addition of vacuum oil on the aging behavior of coal-water slurries.

increase in the viscosity after three weeks of aging. Only after $\mathbf{4 0}$ days of aging did the oilcontaining slurry exhibit a 50 percent increase in its apparent viscosity. The viscosity of slurries prepared with clean coal, on the other hand, nearly doubled when aged to the same extent. Clearly, the addition of vacuum oil results in an even better control of the aging behavior of slurries than that achievable through coal-cleaning.

\section{FREE WATER CONTENT AND THE AGING BEHAVIOR OF SLURRIES}

As discussed earlier in this section, electrokinetic studies indicate that the aging of slurries has only a marginal effect on the coal surface charge, one of the important factors controlling the rheology of coal-water slurries. On the other hand, the rheological behavior of all slurries degrade on aging, the time scale being dependent on the state of oxidation of the coal and the slurry chemistry. Surface chemistry and the solution chemistry controls the immobilization of water molecules, and thereby determine the free water content of slurries, which in turn control the hydrodynamics and the rheological behavior of slurries. We will 
now attempt to quantify the effect of aging on the free water content of slurries and relate the change in the free water content with time to the change in the rheological properties of the slurries.

For a freshly prepared slurry, we may define its fractional free water content, $\Phi_{\mathrm{w}}$, as:

$$
\Phi_{w}=1-\left(\phi_{s} / \phi_{s, \max }\right)
$$

where $\phi_{S}$ is the effective volume fraction of solids in the slurry, and $\phi_{\mathrm{S}, \max }$ is the maximum effective volume fraction of solids achievable. For freshly prepared coal-water slurries the effective volume fractions can be closely approximated as:

$$
\phi_{s}=\frac{w_{s} / \rho_{s}}{\left(w_{s} / \rho_{s}\right)+\left(100-w_{s}\right)}
$$

where $w_{s}$ is the weight percent solids in the slurry and $\rho_{s}$ is the density of the solid. $\phi_{s, \max }$ can be estimated from the relationship bewteen the viscosity and the volume fraction of solids in the slurry. Figure 6.17 shows the relationship between the free water fraction and the viscosity of three different slurries: i) ball mill ground coal and no dispersant, ii) ball mill ground coal with CoalMaster as dispersant, and iii) HPRM/BM ground coal and CoalMaster as dispersant. It is apparent from the figure that there is a power law relationship between the free water content and the viscosity of slurries. Moreover, the three plots overlap each other which suggests that the relationship is independent of the physical as well as the chemical properties.

Several phenomena occur during the aging of slurries, some or all of which may change the free water content of the slurry. If we assume, however, that the relationship between the viscosity and the free water content observed for freshly prepared slurries holds as well for aged slurries, we can estimate the free water content of a slurry as a function of its age from the viscosity data. The free water content of a wide range of slurries is presented as function of their age in Figure 6.18. While it appears from the figure that these slurries differ widely in the relationship between their age and the fraction of free water, it turns out that 


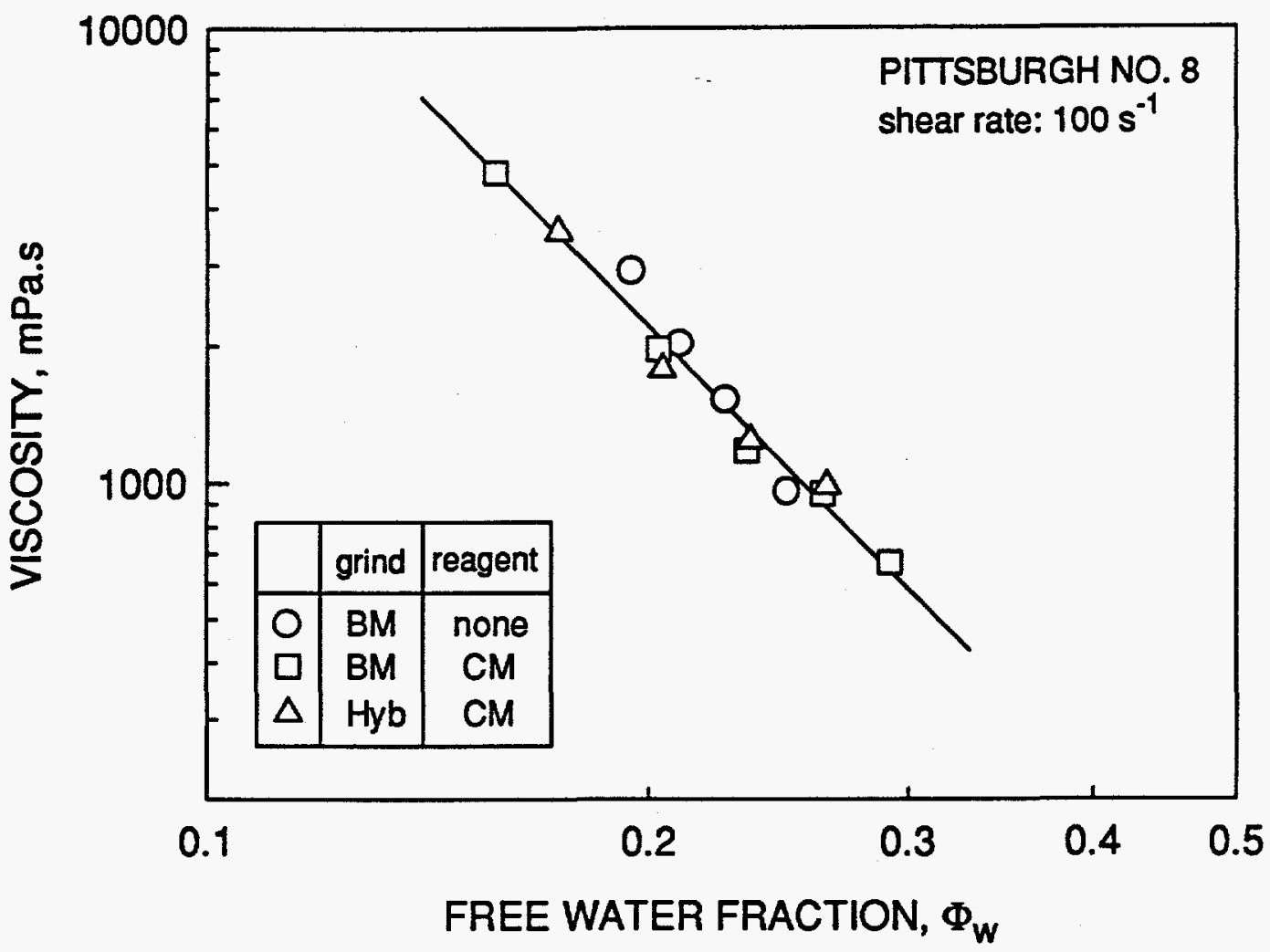

Figure 6.17. The relationship between the free water fraction and viscosity of coal-water slurries.

under suitable scaling the aging behavior of all the slurries are remarkably similar. Figure 6.19 shows the plots of the relative fraction of free water, which is the ratio of the fraction of free water in a slurry and the maximum free water fraction attained during aging, and the age of slurries scaled by the age at which the viscosity of the slurry started to increase.

Earlier in this section, we reported that as the viscosity of slurries increased with age, there was a corresponding decrease in the CoalMaster concentration in the aqueous phase. We now rexamine this phenomena in light of the observed similarity in the aging behavior of all slurries. As can be seen from the results given in Figure 6.20, where the plot of the relative CoalMaster concentration in the aqueous phase as a function of the scaled age of the slurry is superimposed on the plots of the relative free water fraction versus the scaled age, there is indeed a one-to-one correspondence between the free water fraction and the CoalMaster concentration. The relationship is essentially linear, as shown in Figure 6.21. 


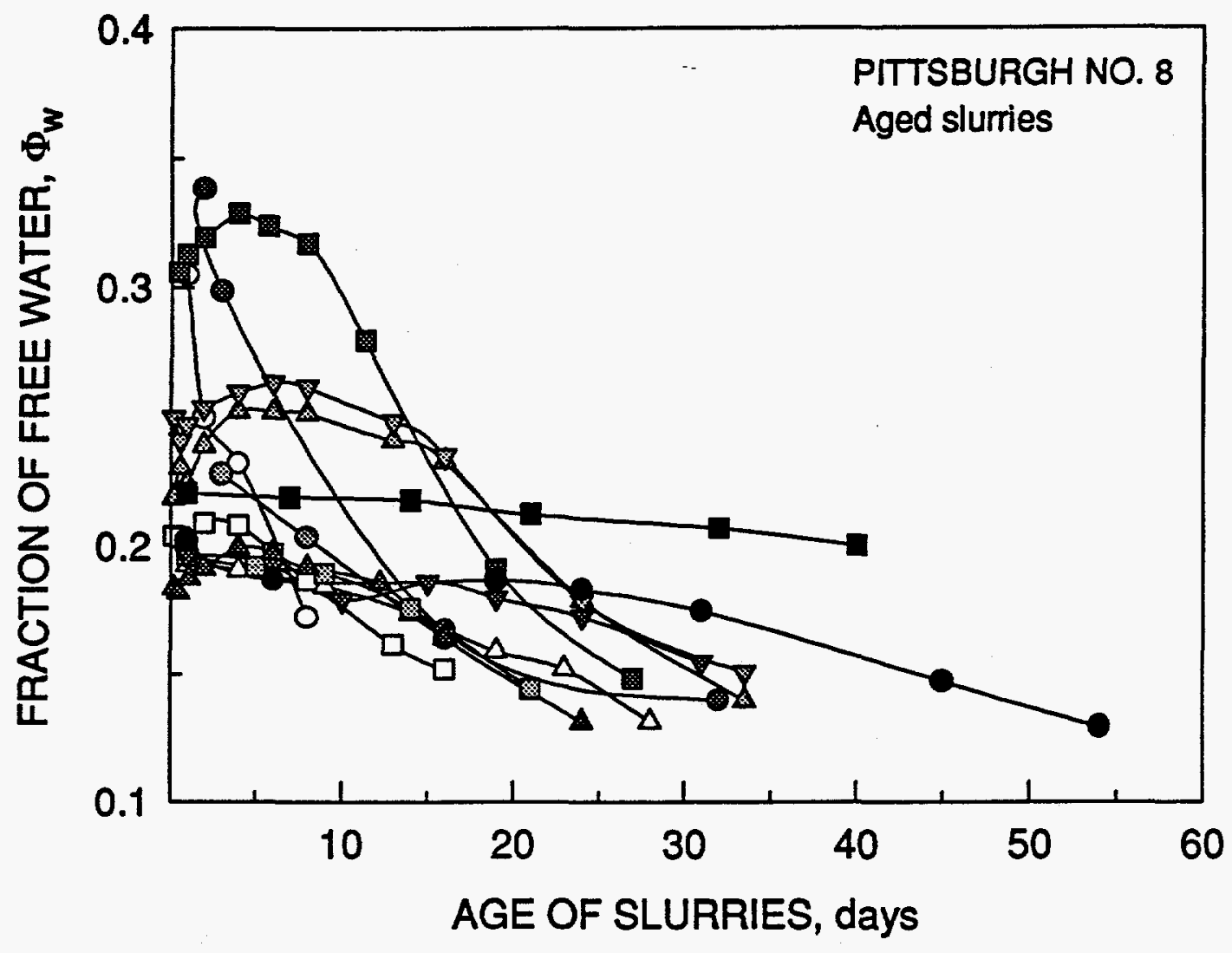

\begin{tabular}{|l|ccl|}
\hline & $\begin{array}{c}\text { wt\% } \\
\text { solids }\end{array}$ & $\begin{array}{c}\text { wt\% } \\
\text { CoalMaster }\end{array}$ & iron control \\
\hline 0 & 65 & 1.0 & none \\
$\square$ & 67 & 2.4 & water-washed \\
$\Delta$ & 67 & 3.0 & none \\
$\nabla$ & 67 & 2.4 & water-washed \\
$\square$ & 67 & 3.0 & water-washed \\
$\Delta$ & 67 & 2.4 & washed with sulfosalicylic acid \\
$\nabla$ & 67 & 2.4 & washed with 2,3-dihydroxypyridine \\
$\square$ & 67 & 1.0 & flotation \\
$\Delta$ & 67 & 1.5 & flotation \\
$\nabla$ & 70 & 3.0 & flotation \\
$\nabla$ & 67 & 3.0 & flotation \\
$\square$ & 67 & 3.0 & tabling and flotation \\
$\square$ & 67 & 1.5 & 1.5 wt\% vacuum oil (+SHMP) \\
\hline
\end{tabular}

Figure 6.18. The effect of aging on the free water content of slurries. 


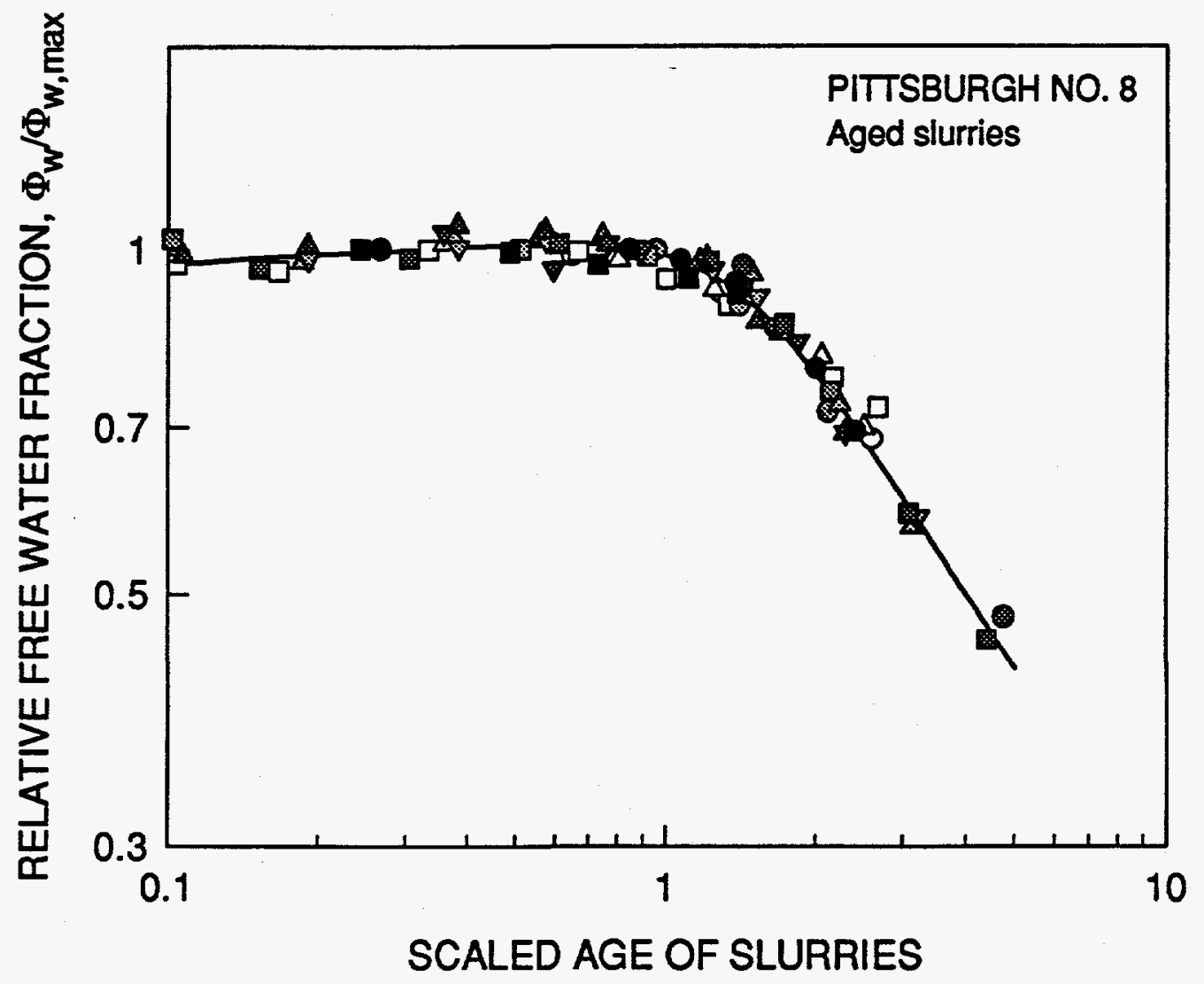

\begin{tabular}{|c|c|c|c|}
\hline & $\begin{array}{l}\text { wt\% } \\
\text { solids }\end{array}$ & $\begin{array}{c}\text { wt\% } \\
\text { CoalMaster }\end{array}$ & iron control \\
\hline 0 & 65 & 1.0 & none \\
\hline 口 & 67 & 2.4 & water-washed \\
\hline$\Delta$ & 67 & 3.0 & none \\
\hline - & 67 & 2.4 & water-washed \\
\hline 贯 & 67 & 3.0 & water-washed \\
\hline$\Delta$ & 67 & 2.4 & washed with sulfosalicylic acid \\
\hline $\boldsymbol{\nabla}$ & 67 & 2.4 & washed with 2,3-dihydroxypyridine \\
\hline - & 67 & 1.0 & flotation \\
\hline 삤 & 67 & 1.5 & flotation \\
\hline$\Delta$ & 70 & 3.0 & flotation \\
\hline $\mathbf{\nabla}$ & 67 & 3.0 & flotation \\
\hline - & 67 & 3.0 & tabling and flotation \\
\hline [ & 67 & 1.5 & 1.5 wt\% vacuum oil (+SHMP) \\
\hline
\end{tabular}

Figure 6.19. Similarity of the aging behavior of slurries. 


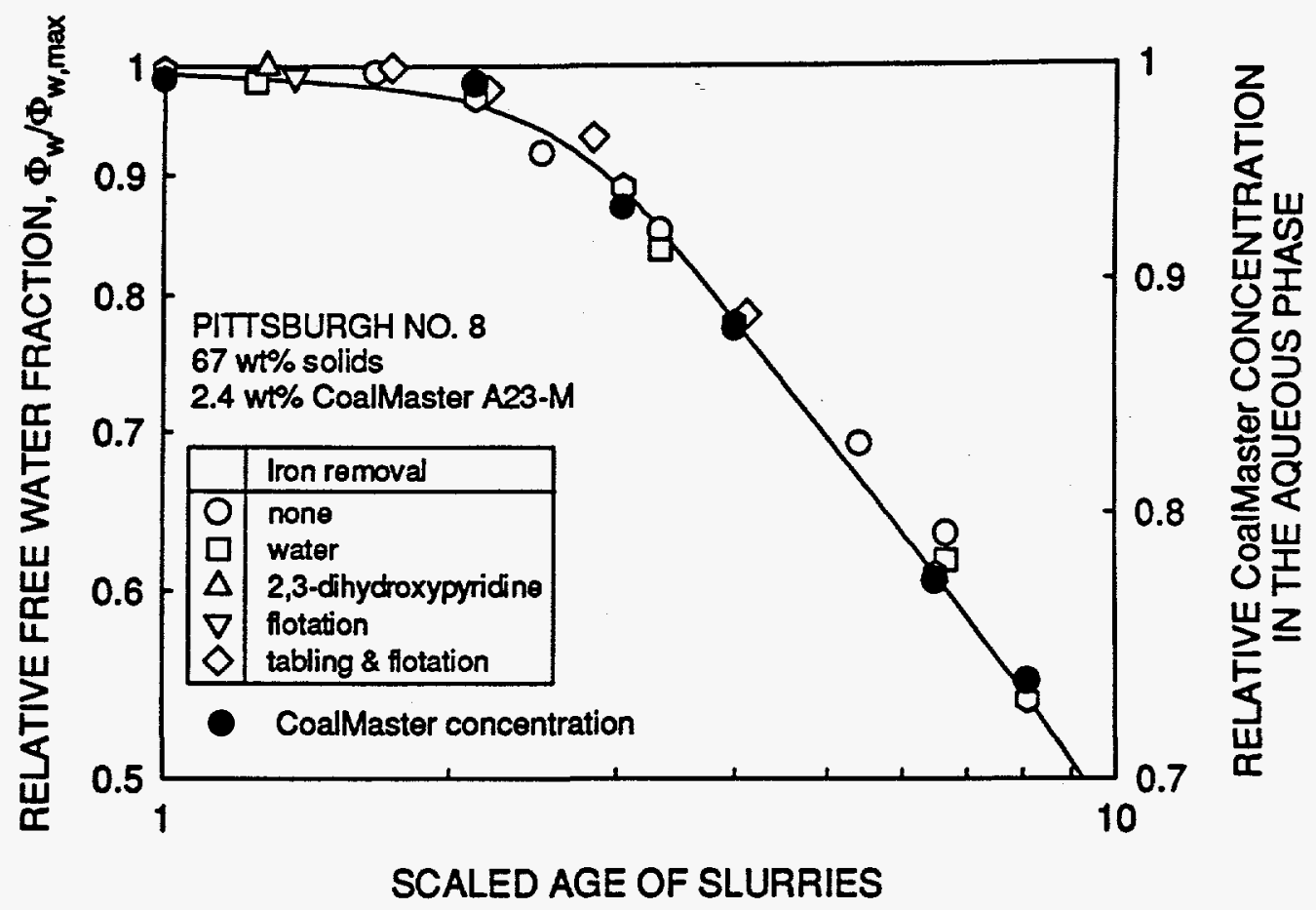

Figure 6.20. The effect of aging on the relative free water content of slurries and the relative CoalMaster concentration in the aqueous phase.

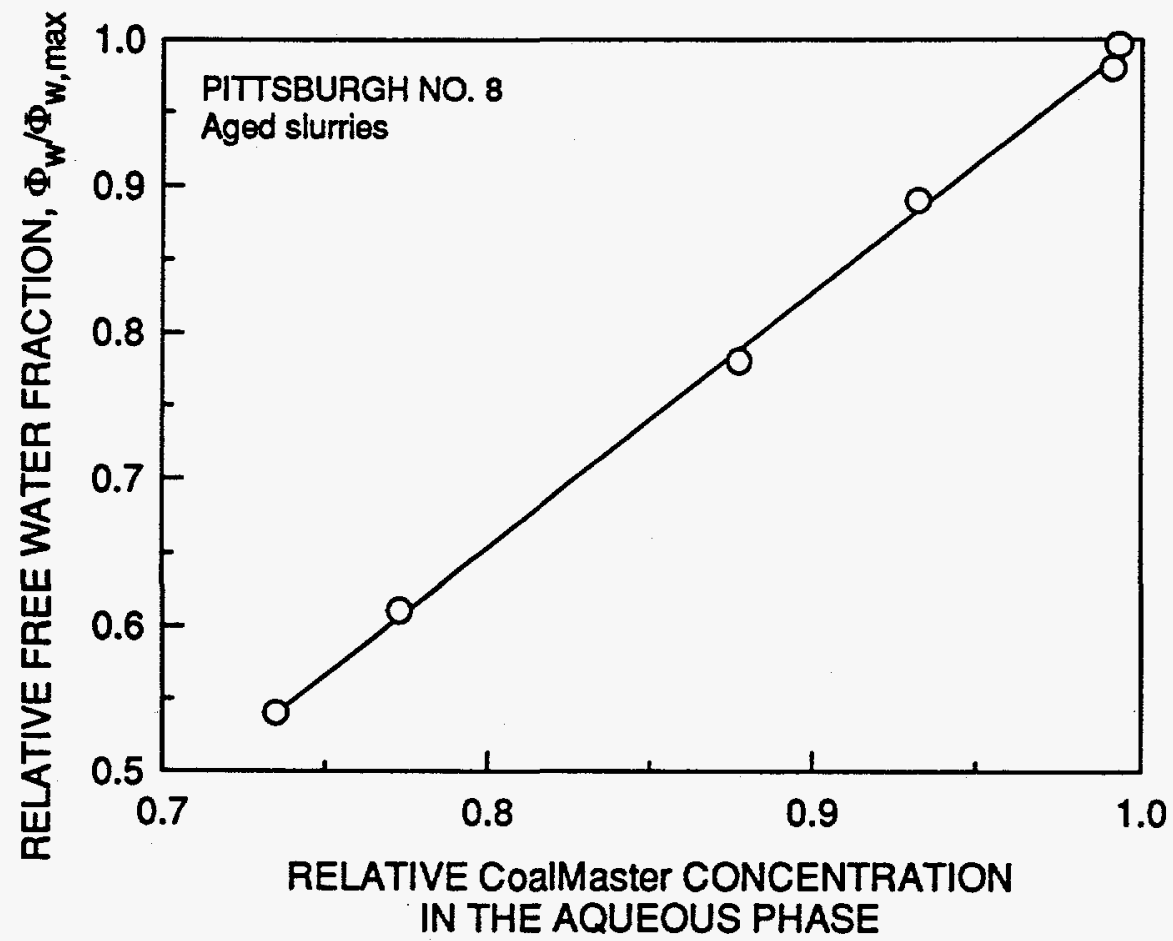

Figure 6.21. Correlation between the relative free water content of aged slurries and the relative CoalMaster concentration in the aqueous phase. 
The relationship between the relative CoalMaster concentration in the aqueous phase and the relative free water content of slurries suggests that a second addition of the dispersant to slurries whose rheological properties have degraded significantly due to aging might result in an improvement in their rheological behavior. A study was therefore undertaken in which a second CoalMaster dosage was added to slurries that had been prepared with unwashed coal and nonionic reagent-washed coal and which were already aged for about a month. The second addition of CoalMaster indeed lowered the viscosity of the aged slurries to that of the freshly prepared ones, as shown by the results given in Figure 6.22. Five days after the second dispersant addition, however, these slurries again exhibited an increase in viscosity, similar to that observed in the case of aging of CWS that had been prepared with coal containing soluble iron. Figures 6.23 and 6.24 present the flow curves and apparent viscosity of the same slurries. It is interesting to note that slurries washed with nonionic reagent exhibit shear thickening behavior after the second addition of the dispersant. Even though the slurry

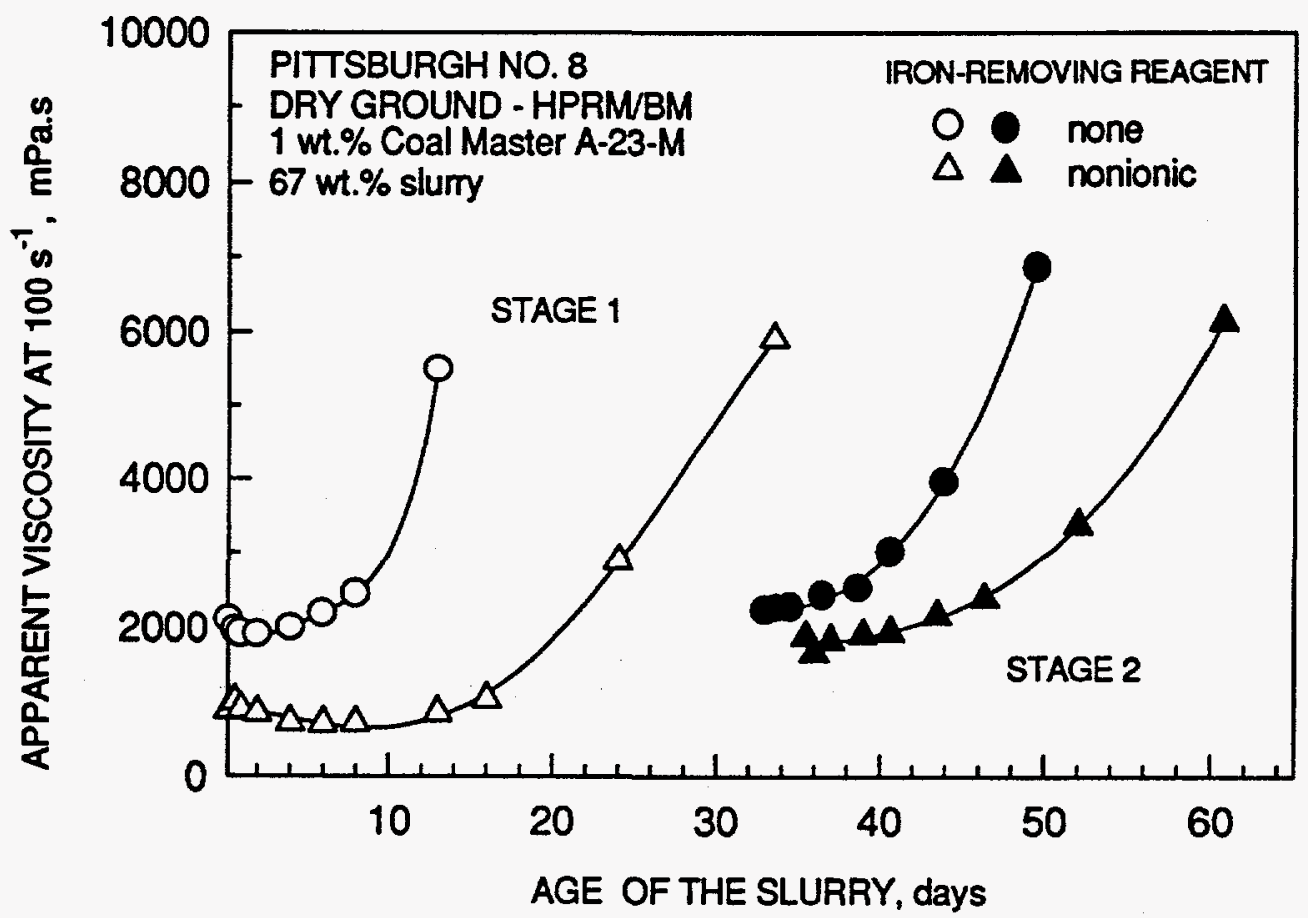

Figure 6.22. Effect of stage-wise addition of CoalMaster on the aging behavior of coal-water slurries. 

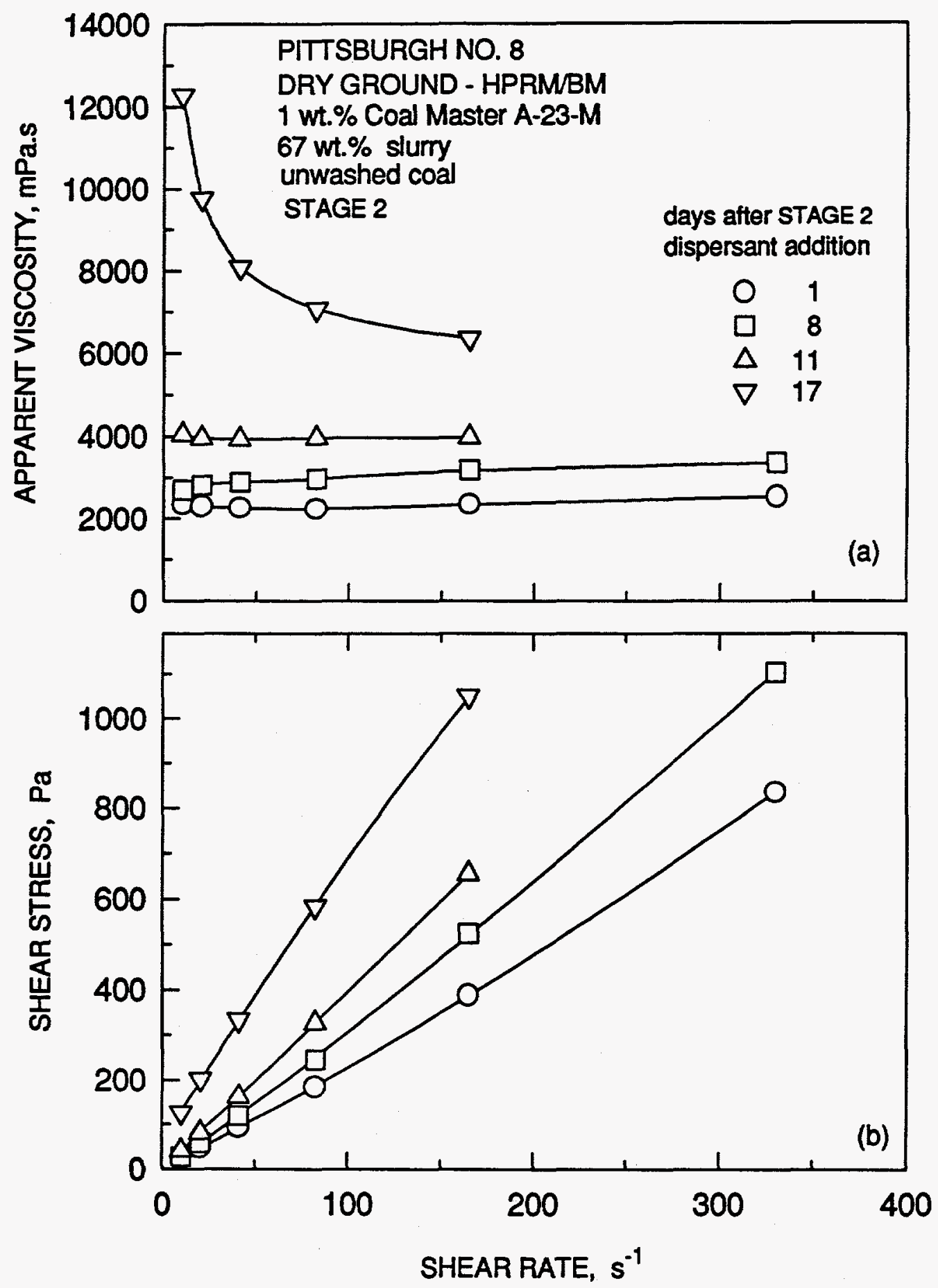

Figure 6.23. Effect of slurry aging in the second stage (second addition of dispersant) on a) the apparent viscosity (measured at different shear rates) and b) the flow curves of the coal-water slurries prepared with unwashed coal. 

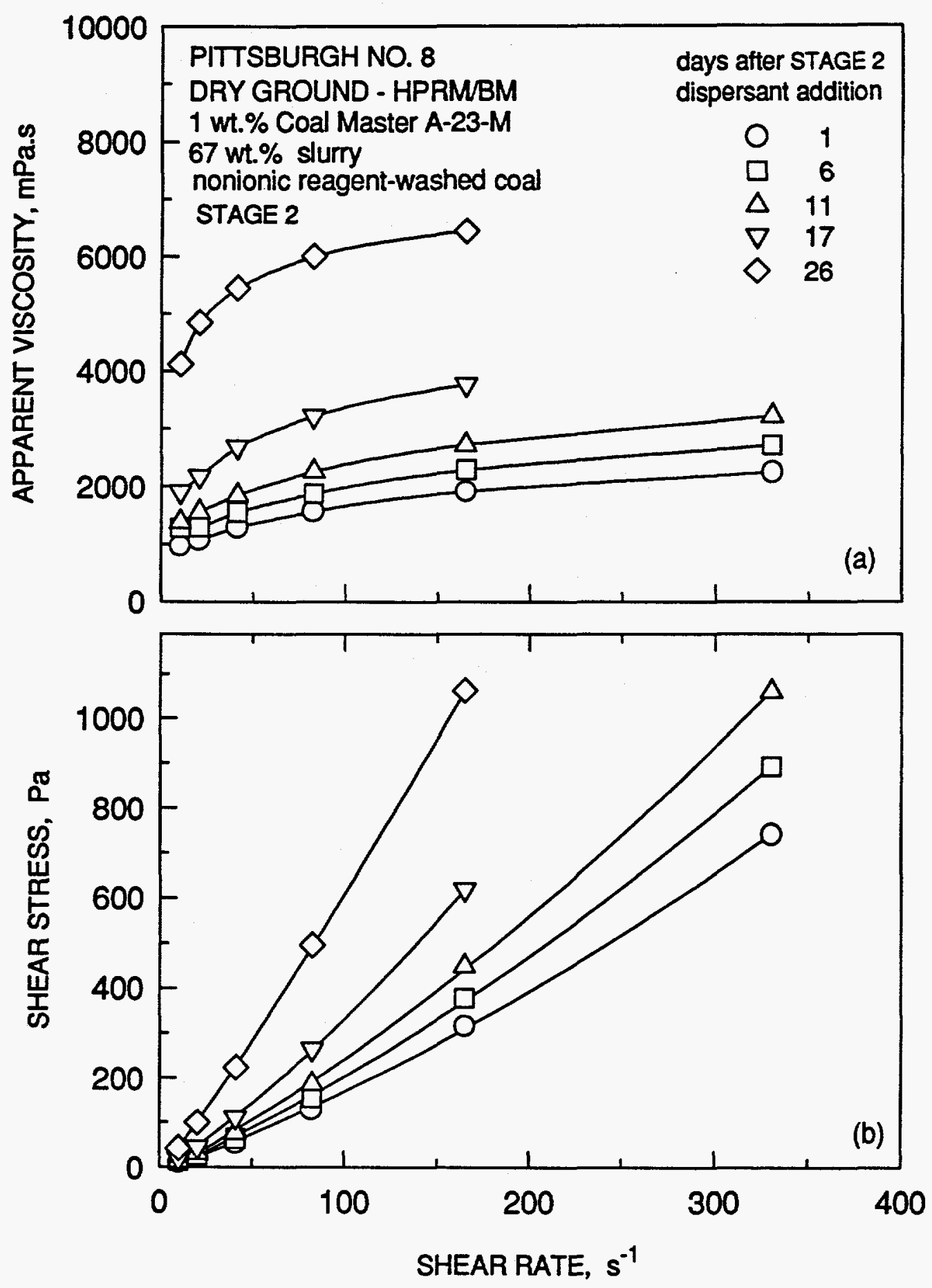

Figure 624. Effect of slurry aging in the second stage (second addition of dispersant) on a) the apparent viscosity (measured at different shear rates) and b) the flow curves of the coal-water slurries prepared with coal washed with 2,3-dihydroxypyridine. 
viscosity was high, no yield stress was observed for that system, which is characteristic of well-dispersed systems. Only a small amount of iron could be detected after stripping it from coal surface with a complexing agent.

\section{SUMMARY}

Study of the aging behavior of slurries showed nonlinear increases in their apparent viscosity over time. This increase was found to be partly due to iron released through the oxidation of the pyrite contained in the coal. This was confirmed through spectroscopic studies and chemical analysis. Removal of iron by washing coal with iron-complexing reagents significantly lowered the apparent viscosity of freshly prepared coal-water slurries and slowed down the degradation of the rheological properties of the slurries, but only temporarily. Physical cleaning of coal, prior to the preparation of slurries, further improved the long-term rheological behavior of the slurries. Addition of vacuum oil to slurries prepared with "as-received" coal and CoalMaster as the primary dispersant resulted in an aging behavior comparable to that of a slurry prepared with clean coal. This is probably because of the reduced rate of oxidation of coal due to the oil film on the particles. A detailed analysis of the aging behavior of slurries showed that the increase in slurry viscosity over time is related to the reduction in the free water content of the slurries. In fact, it turns out that under suitable scaling, all slurries exhibit a remarkably similar relationship between their free water content and their age. 


\section{SECTION VII}

\section{SUMMARY AND CONCLUSIONS}

The objective of this research was the development of improved technology for the preparation of coal-water slurries that have potential for replacing fuel oil in direct combustion. This should be of major importance to the United States in its efforts to reduce dependence on imported oil and to rely more on its enormous low-cost coal resources.

In accordance with this objective, in the first stage of this project, considerable work was conducted to standardize experimental procedures for sample preparation, coal grinding, and rheological measurements to assure reproducibility of the experimental results. Since a Haake RV-12 viscometer with an MV-DIN sensor system was found to give the most reproducible results for measurement of slurry viscosities, this apparatus was subsequently been used for all of our rheological measurements. Methods were developed for applying the acoustophoresis technique for studying the electrokinetic behavior of concentrated coal-water suspensions. These measurements were carried out using this technique to identify the potential of chemical additives for functioning as reagents for effective dispersion.

Detailed investigations of the effect of solids content and chemical additives on the rheology of coal-water slurries, prepared with fines produced by the ball milling of Pittsburgh No. 8 coal, were conducted during the first phase of our research program. These experiments were to provide a baseline against which the rheological behavior of slurries prepared with fines produced by high-pressure roll milling or hybrid high-pressure roll mill/ball mill grinding could be compared. The suitability of various ionic and nonionic reagents as dispersants for the preparation of coal-water slurries were investigated in detail. Of all the reagents tested, a commercially available popular reagent used to prepare the slurries, Coal Master A-23-M from Henkel Corporation, proved to be the most efficient dispersant. CoalMaster was therefore used as the primary dispersant in subsequent studies. 
The aggregative stability of coal-water slurries in the absence and presence of various chemical additives were ascertained by indirect methods, such as the measurement of the relative sedimentation volume and the apparent particle size distributions of the slurries. These studies showed that there is a fairly good correlation between the aggregative stability and the rheological behavior of the slurries.

Wettability studies, through contact angle measurements, showed that CoalMaster significantly improves the wetting of coal by the aqueous phase, even at fairly low concentrations. Viscosity measurements of slurries prepared with different dosages of CoalMaster indicated that there is a direct correspondence between the increase in the wetting of coal particles and the decrease in the viscosity of slurries.

Detailed investigation of the effect of high-pressure roll milling on the energetics of fine grinding and the rheology of coal-water slurries prepared with such fines was carried out in the second stage of the project. Preliminary investigations showed that although the highpressure roll mill grinding of Pittsburgh No. 8 coal resulted in a briquetted product, due to the plastic nature of bituminous coals, deagglomeration of the briquettes and further reduction in particle size could be achieved by grinding the roll mill product in a ball mill with modest additional energy expenditure. Our experimental results indicated that a given degree of size reduction could be achieved by hybrid high-pressure roll mill / ball mill grinding with significantly lower energy expenditure as compared to that required for grinding in a ball mill alone. Viscosity measurements showed that the rheological properties of the slurries prepared with fines produced by the hybrid grinding of coal are better than slurries prepared with fines produced by grinding coal in a ball mill only. Increasing the energy input in the high-pressure roll mill also resulted in an improvement in the rheological properties of slurries. Studies on the effect of grinding environment in the ball mill on the rheology of slurries showed that wet grinding with dispersants results in improved rheological properties.

The viscosity of slurries with high solids content is strongly influenced by the packing density of the feed material. A detailed investigation was therefore carried out to delineate the 
effect of particle size distribution and packing on the rheological behavior of slurries. Our studies showed that for unimodal distribution of particle size, except for a slight deviation at very high solids content where there is significant particle-particle interaction, a power law relationship exists between the viscosity of coal-water slurries and the median size of the solids. Our studies also revealed a direct correspondence between the dry packing density, as determined by tap density measurements, and the maximum achievable solids content of the slurries. Bimodal distributions, generated by mixing coarse and fine distributions, have higher packing densities compared to those of a unimodal distribution with similar median size. The viscosities of slurries, prepared with a bimodally-distributed feed, are also significantly lower than those of slurries prepared with feed having unimodal distribution of identical median size.

Study of the aging behavior of slurries showed nonlinear increases in their apparent viscosity over time. This increase was found to be partly due to iron released through the oxidation of the pyrite contained in the coal. This was confirmed through spectroscopic studies and chemical analysis. Removal of iron by washing coal with iron-complexing reagents significantly lowered the apparent viscosity of freshly prepared coal-water slurries and slowed down the degradation of the rheological properties of the slurries, but only temporarily. Physical cleaning of coal, prior to the preparation of slurries, further improved the long-term rheological behavior of the slurries. Addition of vacuum oil to slurries prepared with "as-received" coal and CoalMaster as the primary dispersant resulted in an aging behavior comparable to that of a slurry prepared with clean coal. This is probably because of the reduced rate of oxidation of coal due to the oil film on the particles. A detailed analysis of the aging behavior of slurries showed that the increase in slurry viscosity over time is related to the reduction in the free water content of the slurries. 


\section{REFERENCES}

1. D. O'Conner and C. D. Harrison, "Coal Utilization," Mining Engineering, Vol. 42, pp. 585-586 (1990).

2. M. J. Hapeman, "Review and Update of Coal Fired Diesel Engine," PWR, Vol. 9, pp. 47-50 (1990).

3. A. White, "Coal-Water Fuel - A New Future for Coal," Pulp and Paper - Canada, Vol. 90, pp. 94-96 (1989).

4. E. P. Olofinskii, "The Problems Involved in Using Coal Slurry as a Fuel at Thermal Power Stations," Thermal Egineering, Vol 36, pp. 699-701 (1989).

5. B. D. Hong, "Coal," Mining Engineering, Vol. 47, pp. 423-426 (1995).

6. N. Berkowitz, An Introduction to Coal Technology, Academic Press, New York (1979).

7. J. ven Brakel and W. Swart, "Technical Feasibility and Energy Costs of Ultrafine Grinding of Coal," Proceedings - First Annual Pittsburgh Coal Conference, University of Pittsburgh, PA, pp. 726-734 (1984).

8. H. Kellerwessel, "High-Pressure Material-Bed Comminution in Practice," Zement-KalkGips, Vol. 43, pp. 57-64 (1990).

9. K. Schönert, "A First Survey of Grinding with High-Compression Roller Mills," International Journal of Mineral Processing, Vol. 22, pp. $401-412$ (1988).

10. G. OberHeuser and S. Strasser, "Roller Press Finish Grinding of Various Products Possibilities and Applications," 7th European Symposium on Comminution, Vol. 2, pp. 789-802 (1990).

11. E. Z. Casassa, G. D. Parfitt, A. S. Rao and E. W. Toor, "The Effect of Surface Active Agents on Coal/Water Slurry Stability," Proceedings of the 6th Intemational Symposium on Coal Slurry Fuels Preparation and Utilization, pp. 251-265 (1984).

12. Leong Yee-Kwong and D. V. Boger, "Surface Chemistry Effects on Concentrated Suspension Rheology," L. Colloid and Interface Science, Vol. 136, pp. 249-258 (1990).

13. P. Taylor, W. Liang, G. Bognolo and Th. F. Tadros, "Concentrated Coal-Water Suspensions Containing Non-ionic Surfactants and Polyelectrolytes 1. A Preliminary Study Using Rheology and Adsorption Isotherms," Colloids and Surfaces, Vol. 61, pp. 147-165 (1991).

14. T. Ogura, M. Tanoura and A. Hiraki, "Behavior of Surfactants in a HIghly Loaded Coal-Water Slurry. I. Effects of Surfactant Concentration on Its Properties," Bull. Chemical Society of Japan, Vol. 66, pp. 1343-1349 (1993). 
15. G. Gabrielli, G. Caminati, E. Camiani, M. Righi and G. Sarti, "The Effect of the SolidLiquid Interface on the Preparation and Properties of Coal-Water Slurries," Journal of Dispersion Science and Technology, Vol. 15, pp. $207-246$ (1994).

16. Th. F. Tadros, P. Taylor and G. Bognolo, "Influence of Addition of a Polyelectrolyte, Nonionic Polymers, and Their Mixtures on the Rheology of Coal/Water Suspensions," Langmuir, Vol 11, pp. 4678-4684 (1995).

17. J. S. Laskowski, "Coal Surface Chemistry and Its Role in Fine Coal Beneficiation and Utilization," Coal Preparation, Vol 14, pp. 115-131 (1994).

18. W. P. Liang and L. Liang, "Effect of Wettability and Adsorbability of Coal on the Slurryability of Coal," Proceedings of the 8th International Symposium on Coal Slurry Euels Preparation and Utilization, p. 19 (1986).

19. H. Atlas, E. Z. Casassa, G. D. Parfitt and E. W. Toor, "The Effect of Cleaning Coal on the Slurryability of Coal," Broceedings of the 7 th International Symposium on Coal Slurry Fuels Preparation and Utilization, p. 1-14 (1985).

20. D. C. Yang, G. Mempel and D. W. Fuerstenau, "A Laboratory Mill for Batch Grinding Experimentation," Trans. SME, pp. 273-276 (1967).

21. D. W. Fuerstenau, J. M. Rosenbaum and Y. S. You, "Electrokinetic Behavior of Coal," Energy and Fuels, Vol. 2, pp. 241-245 (1988).

22. E. Z. Casassa, R. J. Marnicio, G. D. Parfitt, A. S. Rao, E. S. Rubin, H. T. Sommer and E. W. Toor, "A Program of Basic Research on the Utilization of Coal/Water Mixture Fuels," Final Report to the U. S. Department of Energy. Pittsburgh Energy Technology Center. Grant \#DE-FG22-81PC402285, January 1984.

23. E. Z. Casassa, G. D. Parfitt, A. S. Rao and E. W. Toor, in Proceedings of the 7th International Symposium on Coal Slurry Fuels Preparation and Utilization, New Orleans, p. 1, May 1985.

24. H. Polat, M. Polat and S. Chander, "Characterization of Coal Slurries by Electrokinetic Sonic Amplitude (ESA) Technique," Electroacoustics for Characterization of Particulates and Suspensions, Proceedings of workshop held at the National Institute of Standards and Technology, Gaithesburgh, Maryland, S. G. Malghan, Ed., pp.200-218, February 1993.

25. R. W. O'Brien, "Electro-acoustic Effect in a Dilute Suspension of Spherical Particles," J. Fluid Mechanics, Vol. 190, pp. 71-86 (1988).

26. B. J. Marlow, D. Fairhurst and H. P. Pendse, "Colloid Vibration Potential and the Electrokinetic Characterization of Concentrated Colloids," Langmuir, Vol. 4, pp. 611626 (1988). 
27. M. James, R. J. Hunter and R. W. O'Brien, "Effect of Particle Size Distribution and Aggregation on Electroacoustic Measurements of $\zeta$ Potential," Langmuir, Vol. 8, pp. 420-423 (1992).

28. F. N. Desai, H. R. Hammad and K. F. Hayes, "ESA Measurements for Silica and Alumina: Background Electrolyte Corrections," Electroacoustics for Characterization of Particulates and Suspensions, Proceedings of workshop held at the National Institute of Standards and Technology, Gaithesburgh, Maryland, S. G. Malghan, Ed., pp.129-142, February 1993.

29. M. Lindheimer, E. Keh, S. Zaini and S. Partyka, "Interfacial Aggregation of Nonionic Surfactants onto Silica Gel: Calorimetric Evidence," L. Colloid and Interface Science, Vol. 138, pp. 83-91 (1991).

30. G. D. Botsaris and Y. M. Glazman, "Stability and Rheology of Coal Slurries," in Interfacial Phenomena in Coal Technology, G. D. Botsaris and Y. M. Glazman, Eds., Marcel Dekker, Inc., New York, pp. 199-277 (1989).

31. G. Schramm, Introduction to Practical Viscometry, Hakke Viscometers, Hakke Buchles International, Inc., Gebrüder Hakke Gmbh, 119 pp. (1981).

32. Y. L. Chen, S. Chen, C. Frank and J. Israelachvili, "Molecular Mechanisms and Kinetics During the Self-Assembly of Surfactant Layers," J, Colloid and Interface Science, Vol. 153, pp. 244-265 (1992).

33. P. C. Kapur, "Modeling of Tumbling Mill Batch processes," Ch. 9 in Crushing and Grinding Process Handbook, C. L. Prasher, Ed., John Wiley and Sons, pp. 323-363 (1987).

34. D. W. Fuerstenau, P. C. Kapur, K. Schönert and M. Marktscheffel, "Comparison of Energy Consumptions in Breakage of Single Particles in Rigidly Mounted Rolls with Ball Mill Grinding," International J. Mineral Processing, Vol. 28, pp. 109-125 (1990).

35. D. W. Fuerstenau, A. Shukla and P. C. Kapur, "Energy Consumption and Product Size Distributions in Choke-Fed, High-Compression Roll Mills," International Journal of Mineral Processing, Vol. 32, pp. $59-79$ (1991).

36. A. De, "Modeling and Optimization of Fine Grinding of Minerals in High-Pressure Roll Mill - Ball Mill Hybrid Grinding Circuits," Ph.D. Dissertation, University of California, Berkeley, 135 pp. (1995).

37. K. M. Beazley, "Industrial Aqueous Suspensions," in Rheometry: Industrial Applications, Kenneth Walters Ed., Research Studies Press, pp. 348-349 (1979).

38. R. J. Pugh and L. Bergstrom, "Rheology of Concentrated Suspensions," Surface and Colloid Chemistry in Advanced Ceramics Processing, R. J. Pugh and L. Bergstrom Eds., Marcel Dekker, Inc., New York, pp. 199-277 (1994). 
39. J. S. Chong, E. B. Christiansen and A. D. Baer, "Rheology of Concentrated Suspensions," L.Applied Polymer Science, Vol. 15, pp. $2007-2021$ (1971).

40. L. Y. Sadler and G. A. Bethany, "Low Viscosity of Lignite-Water Mixtures," Proceedings of the 7th Intemational Symposium on Coal Slurry Fuels Preparation and Utilization, New Orleans, p. 68, May 1985.

41. R. Kaji, Y. Muranaka, K. Otsuka and Y. Hishinuma, "Effect of Ions on the Rheology of CWM," Proceedings of the 7th International Symposium on Coal Slurry Fuels Preparation and Utilization, New Orleans, p. 16, May 1985.

42. P. D. A. Mills, J. D. Goodwin and B. V. Groover, "Shear Field Modification of Strongly Flocculated Suspensions - Aggregate Morphology," Colloid Polymer Science, Vol. 269, p. $949-963$ (1991). 